NATIONAL LABORATORY

\title{
An Engineering Evaluation of Spherical Resorcinol Formaldehyde Resin
}

September 2010

\author{
Prepared by \\ Joseph Birdwell \\ Denise Schuh \\ Paul Taylor \\ Robert Collins \\ Rodney Hunt
}

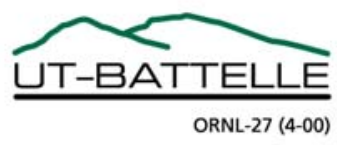




\title{
DOCUMENT AVAILABILITY
}

Reports produced after January 1, 1996, are generally available free via the U.S. Department of Energy (DOE) Information Bridge.

Web site http://www.osti.gov/bridge

Reports produced before January 1, 1996, may be purchased by members of the public from the following source.

\author{
National Technical Information Service \\ 5285 Port Royal Road \\ Springfield, VA 22161 \\ Telephone 703-605-6000 (1-800-553-6847) \\ TDD 703-487-4639 \\ Fax 703-605-6900 \\ E-mail info@ntis.gov \\ Web site http://www.ntis.gov/support/ordernowabout.htm
}

Reports are available to DOE employees, DOE contractors, Energy Technology Data Exchange (ETDE) representatives, and International Nuclear Information System (INIS) representatives from the following source.

Office of Scientific and Technical Information

P.O. Box 62

Oak Ridge, TN 37831

Telephone 865-576-8401

Fax 865-576-5728

E-mail reports@osti.gov

Web site http://www.osti.gov/contact.html

This report was prepared as an account of work sponsored by an agency of the United States Government. Neither the United States Government nor any agency thereof, nor any of their employees, makes any warranty, express or implied, or assumes any legal liability or responsibility for the accuracy, completeness, or usefulness of any information, apparatus, product, or process disclosed, or represents that its use would not infringe privately owned rights. Reference herein to any specific commercial product, process, or service by trade name, trademark, manufacturer, or otherwise, does not necessarily constitute or imply its endorsement, recommendation, or favoring by the United States Government or any agency thereof. The views and opinions of authors expressed herein do not necessarily state or reflect those of the United States Government or any agency thereof. 
Nuclear Science and Technology Division

\title{
AN ENGINEERING EVALUATION OF SPHERICAL RESORCINOL FORMALDEHYDE RESIN
}

\author{
Joseph Birdwell \\ Denise Schuh \\ Paul Taylor \\ Robert Collins \\ Rodney Hunt
}

September 2010

Prepared by the OAK RIDGE NATIONAL LABORATORY

Oak Ridge, Tennessee 37831-6283

managed by

UT-Battelle, LLC

for the

U.S. DEPARTMENT OF ENERGY

under contract DE-AC05-00OR22725 

ORNL/TM-2010/200

DOCUMENT: ORNLTM-2010/200

TITLE: ENGINEERING EVALUATION OP SPHERICAL RESORCINOL FORMALDEHYDE

\section{REVIEWS AND APPROVALS:}

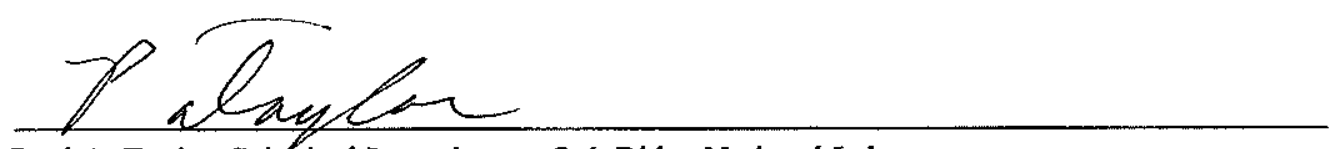

Paul A. Taylor, Prixcipal Investigator, Oak Ridge National Laboratory

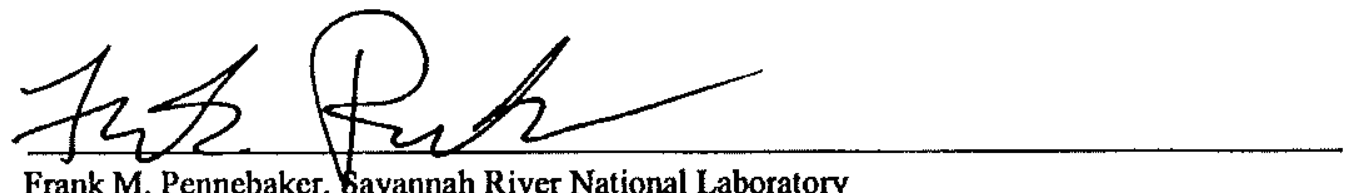

Frank M. Pennebaker, Bavannah River National Laboratory

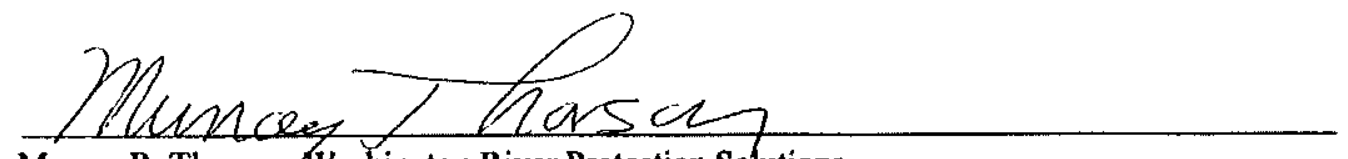

Murray R. Thorson $/$ Washington River Protection Solutions 



\section{CONTENTS}

Page

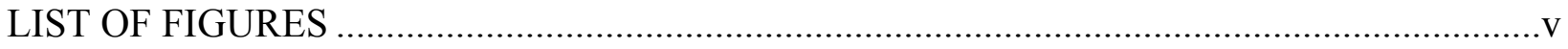

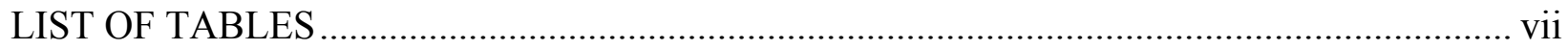

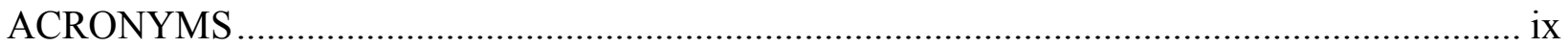

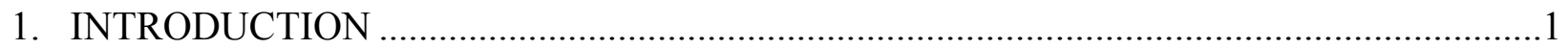

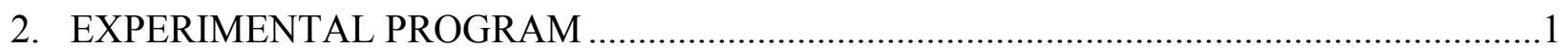

2.1 STANDARD PROCEDURES …........................................................................ 1

2.1.1 Resin Pretreatment and Collection .................................................................2

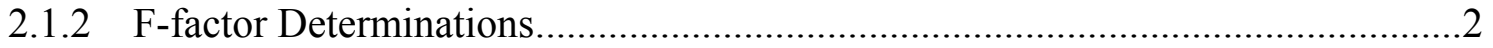

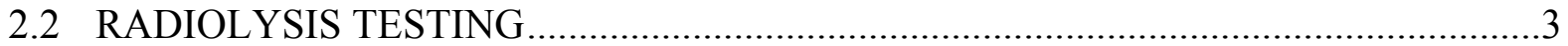

2.2.1 Co-60 Irradiations...................................................................................

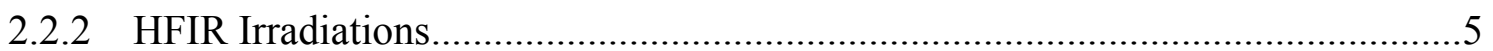

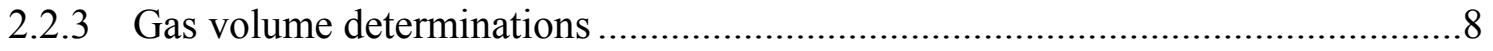

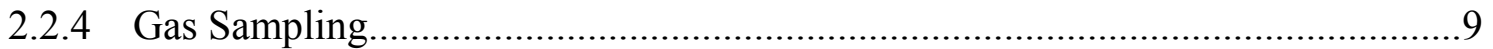

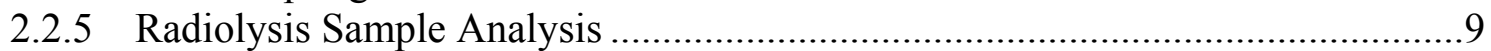

2.3 CESIUM PARTITIONING TEST (K $\mathrm{K}_{\mathrm{d}}$ DETERMINATIONS) ...................................

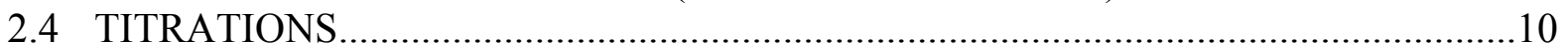

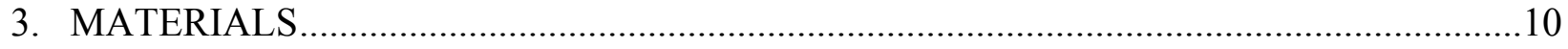

3.1 DISSOLVED SALT SURROGATE COMPOSITIONS ........................................10

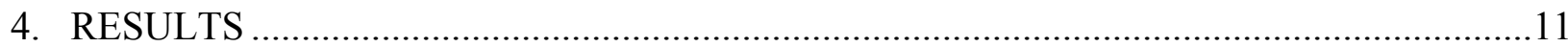

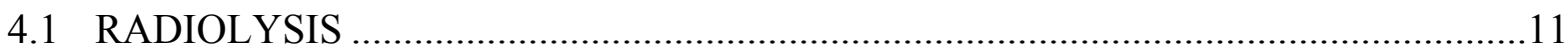

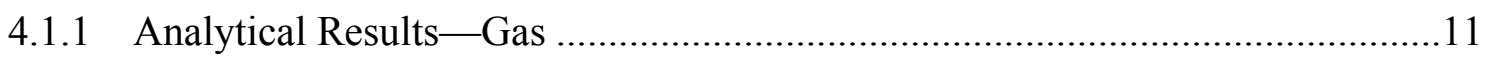

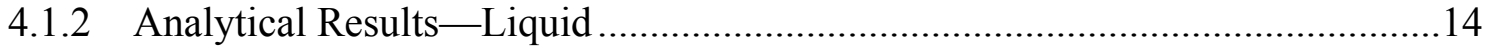

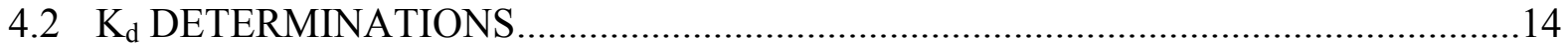

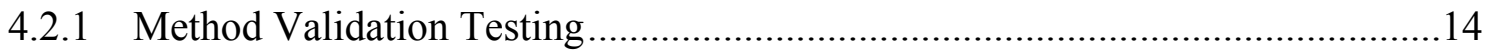

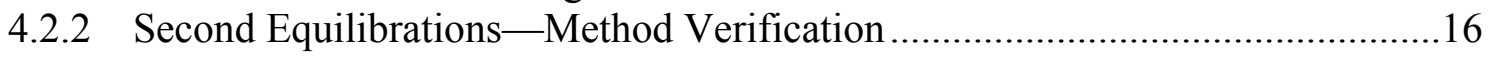

4.2.3 Third Equilibrations_-Method Diagnosis ....................................................16

4.2.4 Fourth Equilibrations — Contacts with AP-101 Simulant..................................19

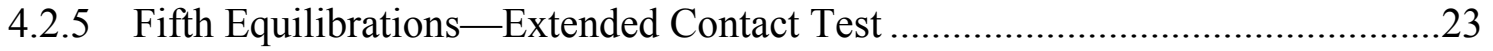

4.2.6 Radiolysis $\mathrm{K}_{\mathrm{d}}$ Tests .................................................................................28

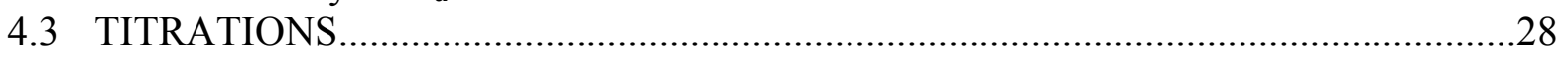

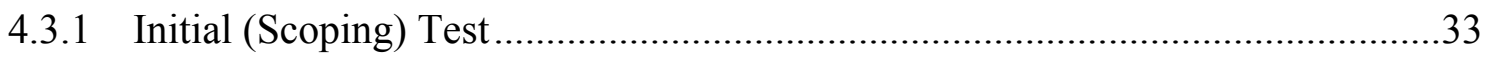

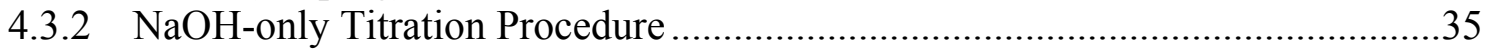

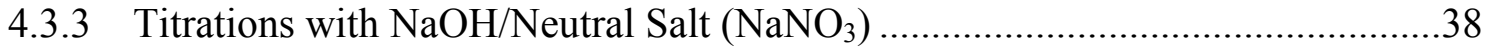

4.3.4 Potassium Loading Results................................................................. 41 


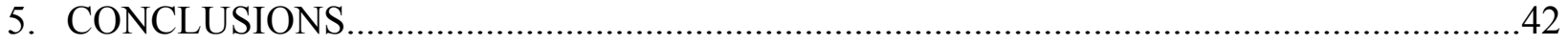

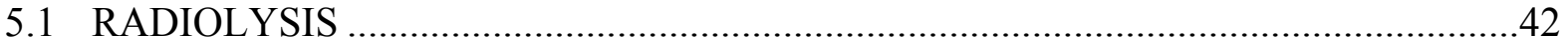

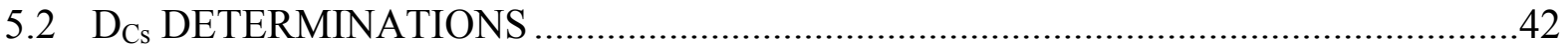

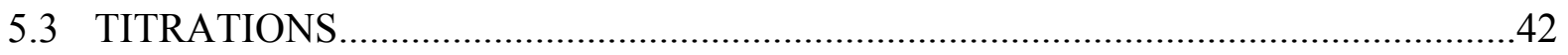

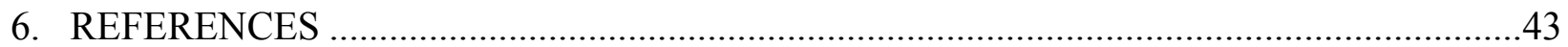

APPENDIX A. ANALYTICAL RESULTS FOR GAS SAMPLES FROM RADIOLYSIS

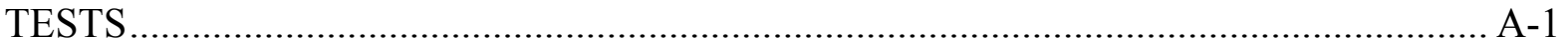

APPENDIX B. ANALYTICAL RESULTS FOR LIQUID SAMPLES FROM RADIOLYSIS

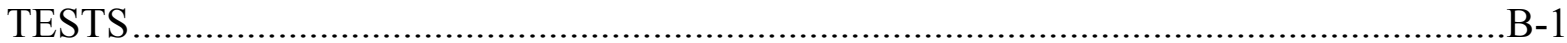




\section{LIST OF FIGURES}

Figure

Page

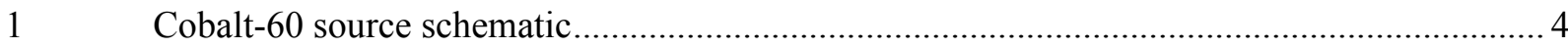

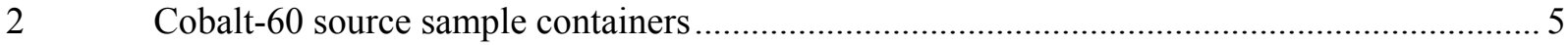

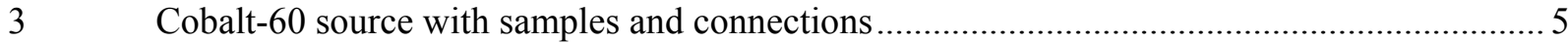

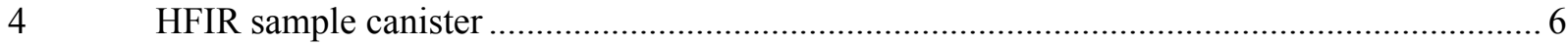

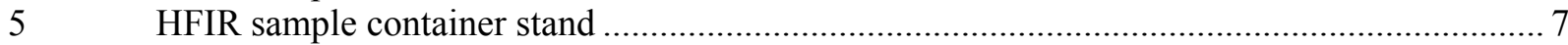

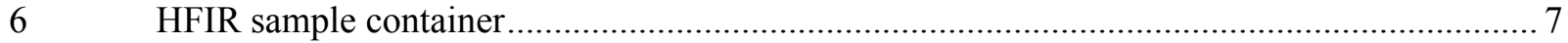

$7 \quad$ Sample canister being lowered into the HFIR spent fuel pool .............................................. 8

$8 \quad$ Sample canister being lowered into a spent fuel element...................................................... 8

9 Comparison of ORNL results from second equilibration test with prior data. (Figure

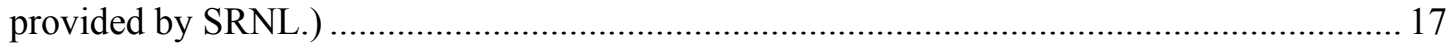

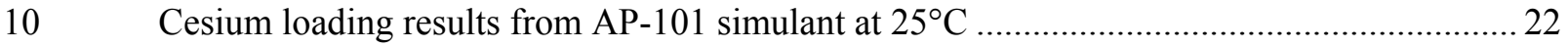

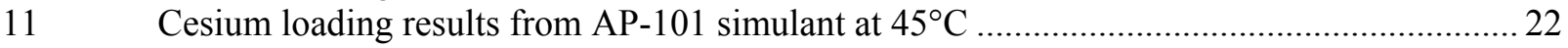

12 Resin and simulant after 3 week contact with AP-101 simulant in $5 \mathrm{M} \mathrm{OH}^{-}$..................... 27

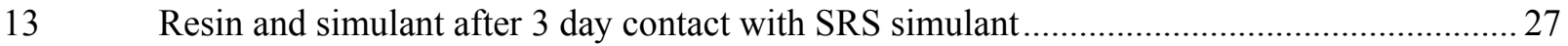

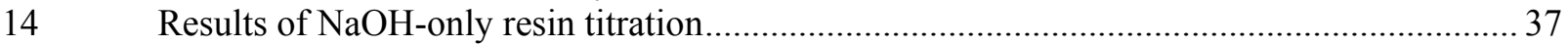

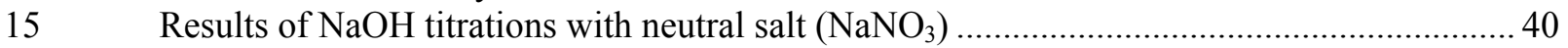

16 Sodium loading (expressed as $\mathrm{OH}^{-}$consumed) versus final $\mathrm{OH}^{-}$concentration ................... 41 



\section{LIST OF TABLES}

Table

Page

1

2

3

4

5

6

7

8

9

10

11

12

13

14

15

16

17

HFIR testing conditions

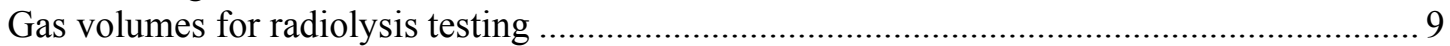

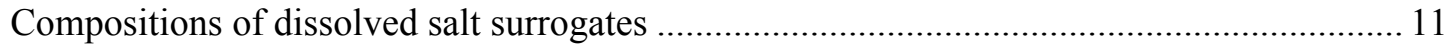

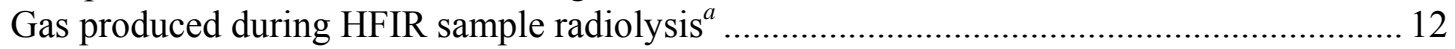

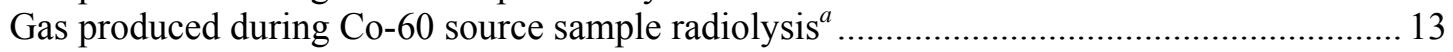

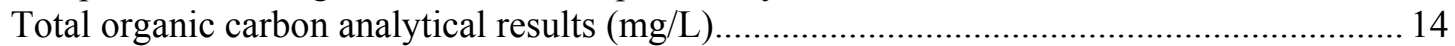

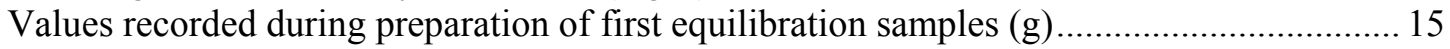

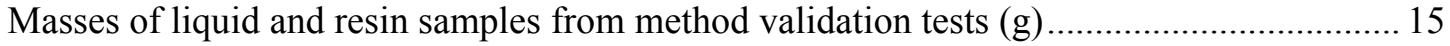

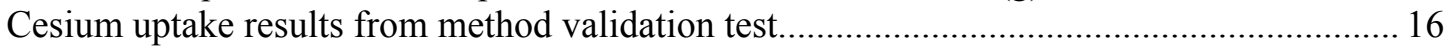

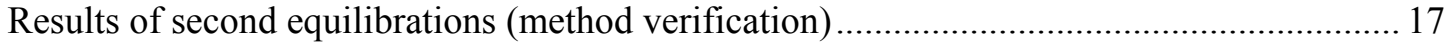

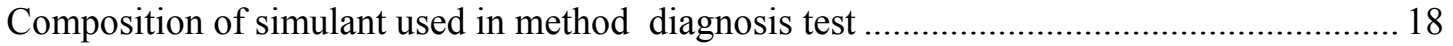

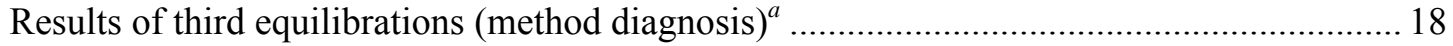

Comparative results from equilibrations performed at SRNL ............................................. 19

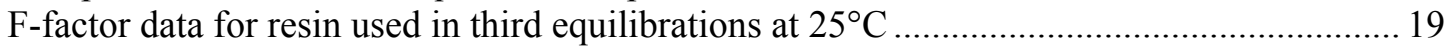

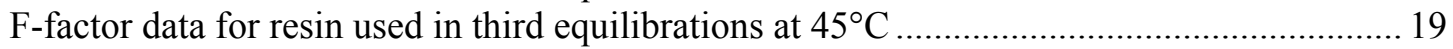

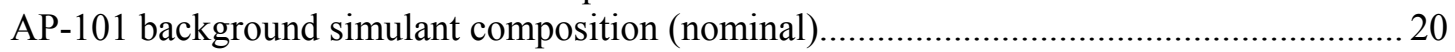

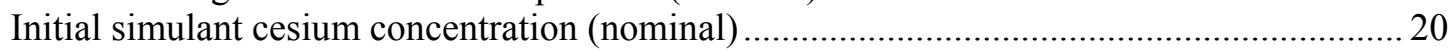

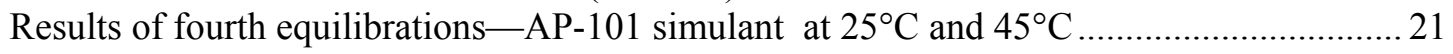

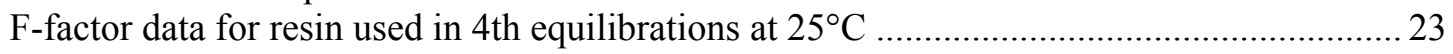

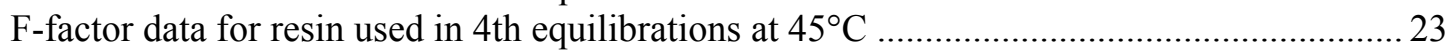

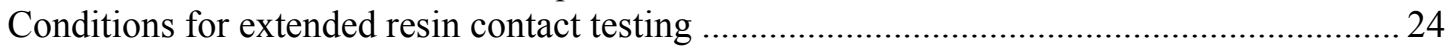

F-factor data for resin used in extended contact equilibrations ........................................... 24

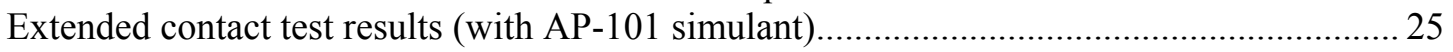

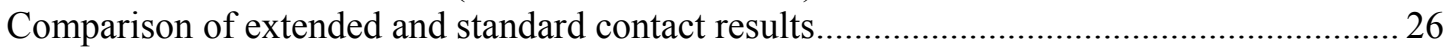

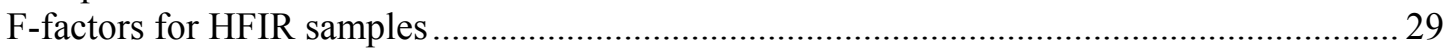

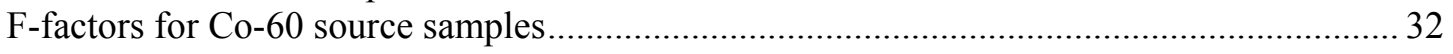

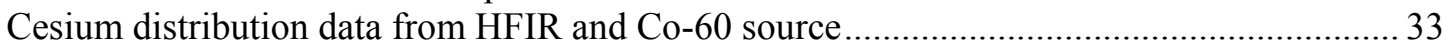

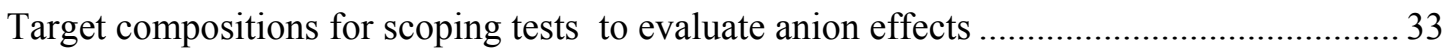

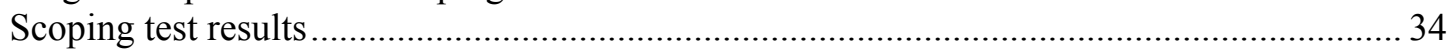

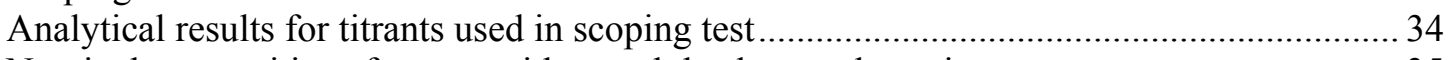

Nominal compositions for tests without salt background matrix .......................................... 35

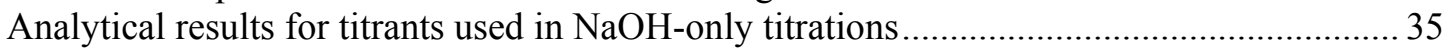

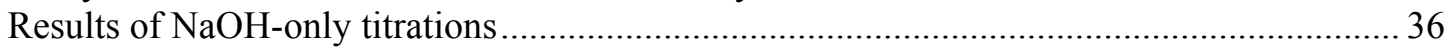

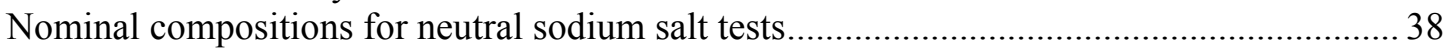

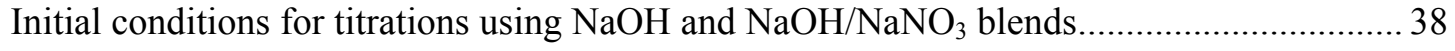

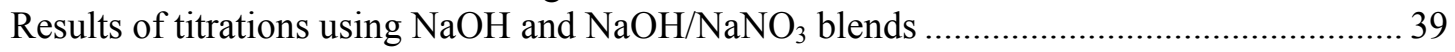

Conditions and results from high ionic strength test with potassium.................................... 42 



\section{ACRONYMS}

$\begin{array}{ll}\text { AES } & \text { atomic emission spectrometry } \\ \text { BV } & \text { bed volume } \\ \text { crystalline silicotitanate } \\ \text { CST } & \text { deionized } \\ \text { DI } & \text { US Department of Energy } \\ \text { DOE } & \text { differential thermal analysis } \\ \text { DTA } & \text { gas chromatograph } \\ \text { GC } & \text { high-density polyethylene } \\ \text { HDPE } & \text { High Flux Isotope Reactor } \\ \text { HFIR } & \text { inductively coupled plasma } \\ \text { ICP } & \text { mass spectrometer } \\ \text { MS } & \text { photoionization detector } \\ \text { PID } & \text { Pacific Northwest National Laboratory } \\ \text { PNNL } & \text { small column ion exchange } \\ \text { RF } & \text { Savannah River National Laboratory } \\ \text { SCIX } & \text { Savannah River Site } \\ \text { SRNL } & \text { semivolatile organic compound } \\ \text { SRS } & \text { thermogravimetric analysis } \\ \text { SVOC } & \text { volatile organic compound } \\ \text { TGA } & \end{array}$





\section{INTRODUCTION}

A small column ion exchange (SCIX) system has been proposed for removal of cesium from caustic, supernatant, and dissolved salt solutions stored or generated from high-level tank wastes at the US Department of Energy (DOE) Hanford Site and Savannah River Sites. In both instances, deployment of SCIX systems, either in-tank or near-tank, is a means of expediting waste pretreatment and dispositioning with minimal or no new infrastructure requirements.

Conceptually, the treatment approach can utilize a range of ion exchange media. Previously, both crystalline silicotitanate (CST), an inorganic, nonelutable sorbent, and resorcinol-formaldehyde (RF), an organic, elutable resin, have been considered for cesium removal from tank waste. More recently, Pacific Northwest National Laboratory (PNNL) evaluated use of SuperLig ${ }^{\circledR}$ 644, an elutable ion exchange medium, for the subject application. Results of testing indicate hydraulic limitations of the SuperLig® resin, specifically a high pressure drop through packed ion exchange columns. This limitation is likely the result of swelling and shrinkage of the irregularly shaped (granular) resin during repeated conversions between sodium and hydrogen forms as the resin is first loaded then eluted. It is anticipated that a similar flow limitation would exist in columns packed with conventional, granular RF resin. However, use of spherical RF resin is a likely means of mitigating processing limitations due to excessive pressure drop. Although size changes occur as the spherical resin is cycled through loading and elution operations, the geometry of the resin is expected to effectively mitigate the close packing that leads to high pressure drops across ion exchange columns.

Multiple evaluations have been performed to determine the feasibility of using spherical RF resin and to obtain data necessary for design of an SCIX process. The work performed consisted of examination of radiation effects on resin performance, quantification of cesium adsorption performance as a function of operating temperature and $\mathrm{pH}$, and evaluation of sodium uptake (titration) as function of $\mathrm{pH}$ and counteranion concentration. The results of these efforts are presented in this report. Hydraulic performance of the resin and the use of eluant alternatives to nitric acid have also been evaluated and have been reported elsewhere (Taylor 2009, Taylor and Johnson 2009).

\section{EXPERIMENTAL PROGRAM}

\subsection{STANDARD PROCEDURES}

Common to the test procedures described in this report were the conversion of resin to the hydrogen form and determination of factors for conversion of damp resin masses to dry resin equivalents (F-factors). The former is necessary in order to establish a consistent basis for expressing resin-loading behavior. Complete conversion of the resin to the hydrogen-loaded form is relatively straightforward and ensures that testing is performed using resin of homogeneous composition. In contrast, the preparation of sodium-form resin includes a final water-washing step that may result in some reconversion of the resin to the hydrogen form, yielding variations in resin properties that introduce error into F-factor values, which, in turn, introduce errors into distribution coefficient values. Determination of factors for converting damp resin mass values to a dry resin basis is required because it is necessary to store resin in liquid and under an inert atmosphere to prevent its oxidation. Subsequently, handling and transfers of resin between storage containers and test apparatuses involve the handling of resin that has had free liquids removed but is not completely dry. 


\subsubsection{Resin Pretreatment and Collection}

Resin preparation was performed according to the protocol in SRNL-RPP-2004-0058 (Nash 2004). Resin used in testing was collected from Batch 5E-370-641, which was received from SRNL on December 2, 2008. Spherical RF resin, which was received in hydrogen form, was rinsed with five bed volumes $(\mathrm{BV})$ of deionized (DI) water for at least 30 minutes and the slurry was agitated approximately every 10 minutes. The liquid was decanted in a manner that minimized resin contact with air and was then contacted with $5 \mathrm{BV} 1.0 \mathrm{M} \mathrm{NaOH}$ for at least $16 \mathrm{~h}$, during which time it was agitated periodically. The liquid was again decanted, and the resin was contacted with 3 BV DI water for at least 30 minutes and agitated periodically. The wash liquor was decanted, and a second and a third wash with 3 BV of DI water were performed. Preparation of sodium-form resin was completed with the third wash, leaving the resin in sodium form. For the hydrogen-form resin, the third water wash was decanted and $10 \mathrm{BV}$ of 0.5 $\mathrm{M}$ nitric acid was transferred into the resin container. The acid-resin slurry was agitated periodically during a contact time of at least $2 \mathrm{~h}$. The acid solution was decanted from the container, and the resin was washed with three batches of $3 \mathrm{BV}$ DI water in the manner performed after the previous contact with $\mathrm{NaOH}$. The resin was stored in the third rinse solution under an inert atmosphere (argon). Tape was placed over the joint between the lid and bottle to ensure an airtight seal.

Prior to each set of experiments, volumes of resin adequate for the test procedure and for F-factor determinations were collected from the pretreated resin that was stored in the desired state (sodium or hydrogen form) under liquid and inert gas (argon).

Resin for all tests other than the method validation equilibrations (a subset of the cesium loading determinations) was collected by pouring slurried resin directly into a Buchner funnel into which a Whatman 41 filter disc had been placed. The filter was prewetted with DI water to seal the filter paper to the funnel. Vacuum was initiated after wetting the filter and prior to sample transfer. As soon as transfer of resin to the filter was completed, a Petri dish modified with a hose nipple was placed over the funnel to serve as a lid, and argon was passed over the resin and through the filter as liquid was being removed. Vacuum was maintained for at least 2 minutes after the last drop of free liquid was observed to fall from the filter. When smaller numbers of resin samples were collected for use in $\mathrm{K}_{\mathrm{d}}$ determinations, titrations, or radiolysis tests, associated samples for use in F-factor determinations were collected as the first and last resin samples. When larger sets of samples were collected (more than six samples), collection of material for F-factor determination was interspersed throughout the resin collection process. This approach was intended to compensate for incidental water loss that may have occurred after vacuum filtration was completed (i.e., during the process of collecting and weighing of filtered resin samples), which would generate a biased-low F-factor value. Due to the use of resin samples in F-factor measurements and equilibrations immediately after collection, samples collected for those purposes were not purged with argon for transfer to the vacuum furnace or to equilibration flasks.

In the case of the method validation test, water removal was performed under normal atmosphere (i.e., not under inert gas). In addition, volumes of resin for this test and the associated F-factor determination were removed from the supply container by submerging the larger-diameter end of a glass pipette into the supply container, allowing resin slurry to fill the end of the tube, then sealing the other end of the tube to prevent venting.

\subsubsection{F-factor Determinations}

With the exception of one set of cesium adsorption determinations (the method validation test), F-factor determinations were conducted in the same manner. Initial masses of damp acid-form resin samples collected for use in F-factor determinations subsequent to the method validation test were in the range of 1.0 to $1.2 \mathrm{~g}$ each. Sample containers were labeled and weighed prior to transfer of resin. Resin was added, and the net mass was determined incrementally until the value reached the target mass range. The sample containers were then capped, and the gross mass of each container was measured. Resin samples were transferred to a vacuum furnace, where they were reopened prior to being heated to a 
nominal temperature of $50^{\circ} \mathrm{C}$. Heating was maintained in the range of $50^{\circ} \mathrm{C}$ to $55^{\circ} \mathrm{C}$ under a pressure of not more than 60 Torr (absolute). Determinations made in conjunction with the second and third $\mathrm{K}_{\mathrm{d}}$ determination tests (i.e., the two tests following method validation) were performed with no materials other than the resin samples and their containers in the furnace during drying. In the remaining tests, a beaker containing a dessicant (anhydrous calcium sulfate) was placed in the furnace to trap moisture released from the damp resin. The added desiccant significantly accelerated the drying process.

The resin samples were periodically removed from the furnace, capped, and weighed, and the masses were recorded. In all cases, the intervals between consecutive weighings were at least $3 \mathrm{~h}$.

Heating/weighing cycles were repeated until no mass changes at the milligram level were observed after three consecutive weighings.

The method for determining F-factors that was used in conjunction with the method validation test is described in Sect. 4.2.1 of this report.

\subsection{RADIOLYSIS TESTING}

Radiolysis testing focused on irradiating samples of RF resin under various conditions to determine whether the RF resin would degrade when exposed to a radiation field. Samples of RF were irradiated in various solutions that the resin would be exposed to during normal use, including an average simulant for Savannah River Site (SRS) supernatant in tanks 1, 2, and 3, a simulant for Hanford tank AP-101, water, and $0.5 \mathrm{M} \mathrm{HNO}_{3}$. The gamma dose to the samples ranged from $50 \mathrm{Mrad}$ to $300 \mathrm{Mrad}$. Large volumes of resin were irradiated in a Co-60 source to levels ranging from 100 to $300 \mathrm{Mrad}$; smaller volumes were irradiated to levels ranging from 50 to 300 Mrad in ORNL's High Flux Isotope Reactor (HFIR). Radiation effects were evaluated by two methods: collection and analysis of gas samples collected from the headspace over the liquid/resin samples and determination of cesium distribution coefficients for irradiated resin. The small volumes allow for $\mathrm{K}_{\mathrm{d}}$ tests to be performed at each specified dose, while the large volume samples had $\mathrm{K}_{\mathrm{d}}$ tests performed only at the endpoint, $300 \mathrm{Mrad}$, which will be reported in Sect. 4.2.6, "Radiolysis $\mathrm{K}_{\mathrm{d}}$ Tests."

Common to all radiolysis test samples was the determination of gas volumes generated due to resin and solution degradation and the analysis of gas and liquid samples for resin degradation products: hydrogen, oxygen, nitrogen, nitrous oxide, carbon monoxide, carbon dioxide, and volatile organic compounds (VOCs).

\subsubsection{Co-60 Irradiations}

Preconditioned RF was dewatered using filter paper and was transferred into a graduated cylinder by sluicing using the target solution (i.e., SRS simulant, water, or $0.5 \mathrm{M} \mathrm{HNO}_{3}$ ). The amounts of RF and solution were adjusted so that the total depth of the liquid and resin was $40 \%$ greater than the depth of the settled resin. The volume of resin was approximately $330 \mathrm{~mL}$; the liquid volume was $220 \mathrm{~mL}$. Irradiations under water and SRS waste simulant were performed using sodium-form resin; hydrogen-form resin was irradiated under $0.5 \mathrm{M} \mathrm{HNO}_{3}$. In each case, the mass of the irradiated resin was calculated from the initial resin volume and previously determined resin bulk densities: $0.368 \mathrm{~g} / \mathrm{mL}$ for $\mathrm{H}$-form resin and $0.297 \mathrm{~g} / \mathrm{mL}$ for sodium form resin (Fiskum 2006).

Unirradiated control samples were prepared by placing resin under SRS simulant, water, and $0.5 \mathrm{M}$ $\mathrm{HNO}_{3}$. In each case, the solution of interest was added to $10 \mathrm{~mL}$ of preconditioned RF until the total sample volume was $16.7 \mathrm{~mL}$. The samples were subjected to the same chemical exposure conditions (contact duration, handling and temperature) as the radiolysis samples.

After RF resin samples were prepared at ORNL and transferred into stainless steel containers, they were sent to SRNL for radiolysis up to $100 \mathrm{Mrad}$. Irradiation sample containers were vented during irradiation at SRNL. Preliminary irradiations were performed at SRNL because the SRNL Co-60 source has a more intense gamma field, thus shortening the irradiation time for a $100 \mathrm{Mrad}$ dose to 2 weeks from the 4 months that would be required using the ORNL Co-60 source. The maximum temperature indicated 
during the SRNL irradiations was $30^{\circ} \mathrm{C}$. The control samples were placed in a warm water bath for 14 days to simulate temperature conditions during the SRNL irradiations.

Following irradiation at SRNL, the sample containers were returned to ORNL and were placed into the Co-60 source in Building 4501. After the three sample chambers were returned from SRNL, they were connected to the gas-sampling port using separate gas lines. A digital pressure gauge was included in each line. The idea was to record the pressure in each line manually each day, but the pressure gauges would only run for 2 weeks before the batteries needed to be changed. The batteries were not replaced because the pressure gauges could have reset.

The lines were connected to a sampling port, which included a baratron pressure transducer, vacuum pump, and a Cajon fitting. The volume of the sampling port was determined with the use of the 2-L expansion port. A schematic of the apparatus and connections can be seen in Fig. 1; a picture of the sample containers is provided in Fig. 2, and a picture of the sample and connectors in the irradiator is provided in Fig. 3.

The samples were lowered into the irradiator. At $50 \mathrm{Mrad}$ intervals from 150 to $300 \mathrm{Mrad}$, gas samples (approximately $10 \mathrm{~mL}$ each) were collected from each sample container and were analyzed. After each gas grab sample, the sample container in the irradiator was vented. All lines above the double valves were evacuated after each sample. At the end of the radiolysis, liquid samples were obtained and tested for semivolatile organic compounds (SVOCs) and formaldehyde. The resin was also collected, and $\mathrm{K}_{\mathrm{d}}$ tests were performed in triplicate. The results were compared with the results for the control samples.

Previous experiments have been performed from 0-100 Mrad at SRS and PNNL (Crawford 1993, Bibler 1994, and Carlson 1995). The purpose of the Co-60 source testing is to expand upon the previous experiments.

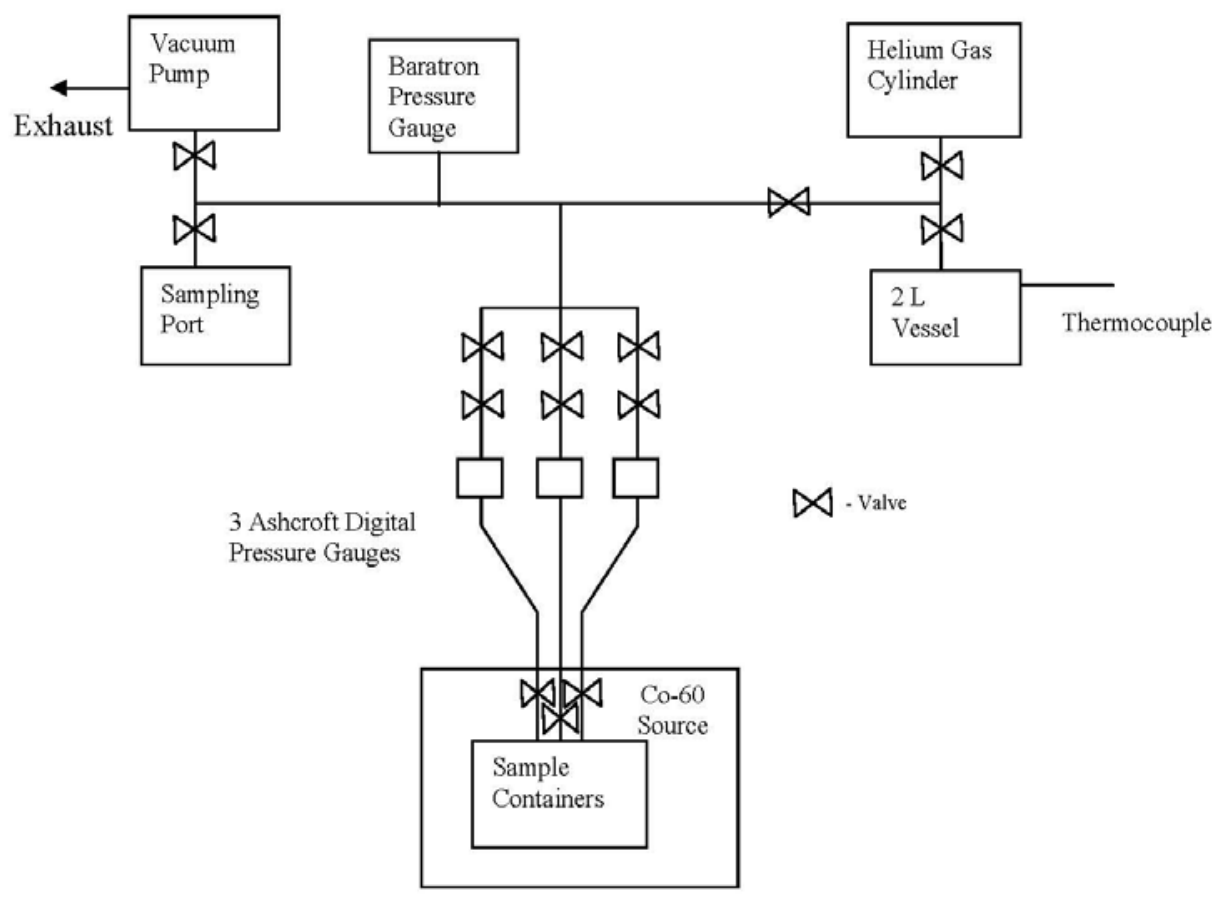

Fig. 1. Cobalt-60 source schematic. 


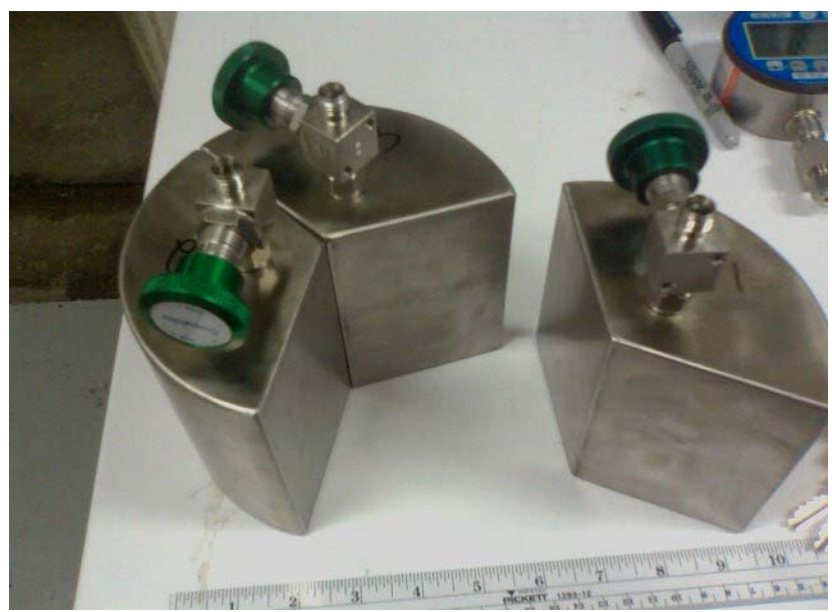

Fig. 2. Cobalt-60 source sample containers.

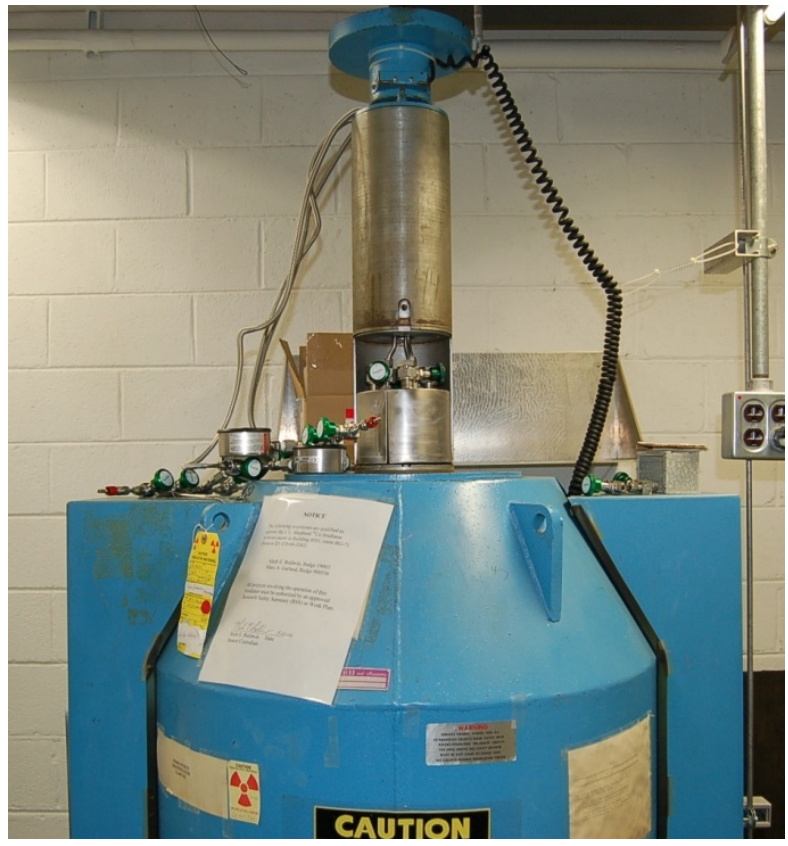

Fig. 3. Cobalt-60 source with samples and connections.

\subsubsection{HFIR Irradiations}

Preconditioned RF was transferred into a graduated cylinder with the aid of the target solution (i.e., SRS, Hanford, water, or $0.5 \mathrm{M} \mathrm{HNO}_{3}$ ). As in the Co-60 irradiations, the amounts of RF and solution were adjusted so that the depth of the slurry was $40 \%$ greater than the depth of the settled resin. The volume of resin in each sample was approximately $83 \mathrm{~mL}$; the liquid volume was $52 \mathrm{~mL}$. The combined sample was loaded into a $150 \mathrm{~mL}$ HOKE® stainless steel sample bottle. The void space (nominally $15 \mathrm{~mL}$ ) was minimized to facilitate generation of pressure adequate for the subsequent transfer of gas into containers for shipping to the analytical laboratory. As with the Co-60 sample irradiations, the masses of the resin were determined using previously determined bulk densities for $\mathrm{H}$-form resin and $\mathrm{Na}$-form resins. Irradiations under $\mathrm{HNO}_{3}$ were performed using $\mathrm{H}$-form resin; irradiations under water, SRS simulant, and 
Hanford simulant were performed using Na-form resin. Based on the previously stated bulk densities for the resin in these forms (Fiskum, 2006), the dry-basis resin mass of $\mathrm{H}$-form samples was 19.14g and the approximate mass of resin in Na-form samples was $15.45 \mathrm{~g}$.

Irradiations were performed by placing the resin samples into the HFIR spent fuel pool. Individual samples containing virgin resin were irradiated to doses of 150, 200, 250, and $300 \mathrm{Mrad}$. Additional samples of virgin resin and oxidized material (with an oxygen uptake of $7 \mathrm{mmoles}$ oxygen/g of dry resin) submerged in water were irradiated to doses of 50 and $100 \mathrm{Mrad}$. The oxidized material had the same pretreatment as the virgin resin, but was in contact with Hanford simulant with a known oxygen exposure when it was used in the hydraulic conductivity test loop for several weeks.

The testing conditions for the HFIR experiments are presented in Table 1.

Table 1. HFIR testing conditions

\begin{tabular}{lcl}
\hline \multicolumn{1}{c}{ Liquid } & Type of resin & \multicolumn{1}{c}{ Exposures [Mrad] } \\
\hline Water & New & $50,100,150,200,250,300$ \\
Water & Used & 50,100 \\
$0.5 \mathrm{M}$ nitric acid & New & $150,200,250,300$ \\
Hanford simulant & New & $150,200,250,300$ \\
SRS simulant & New & $150,200,250,300$ \\
\hline
\end{tabular}

The sample containers were placed into HFIR sample canisters (Fig. 4), which are stainless steel cylinders that fit inside HFIR's cylindrical fuel assembly. The sample canister has a vent line and an inert gas supply line. The interior dimensions of the HFIR sample can are 24 in. long and 3 in. inner diameter.

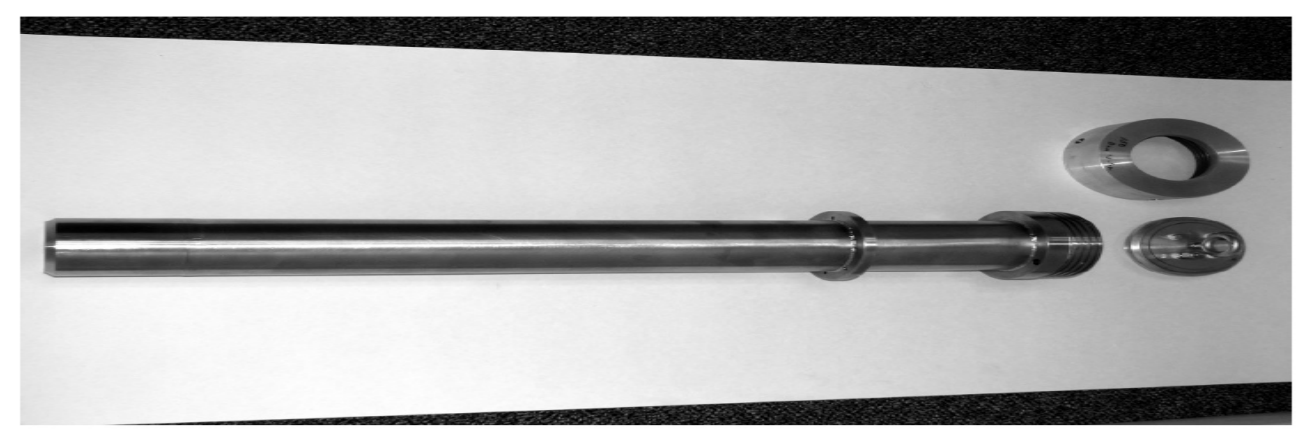

Fig. 4. HFIR sample canister.

Per HFIR operating requirements, no plastic or polymeric materials (as would be used in O-rings) can be placed inside a HFIR sample canister for irradiation due to the high probability of failure from gamma radiolysis. Accordingly, the inner Hoke ${ }^{\circledR}$ container that was selected for use does not utilize polymer or elastomer materials. As stated previously, the container was sized so that the sample would occupy $90 \%$ of the canister volume to allow room for gas expansion while allowing sufficient pressure buildup to facilitate gas sample collection.

Also per HFIR requirements, rupture disks were installed to protect against overpressure of the internal sample container. In the event of an overpressure, disk rupture would have resulted in venting of the internal container into the HFIR canister. The rupture disks used on sample vessels containing SRS and Hanford simulants were fabricated from Inconel 600; vessels containing resin under water or nitric acid were made of tantalum. All rupture discs were fabricated to fail at $300 \mathrm{psig}$. A picture of the sample container stand and a picture of the sample container can be seen in Fig. 5 and Fig. 6, respectively. 


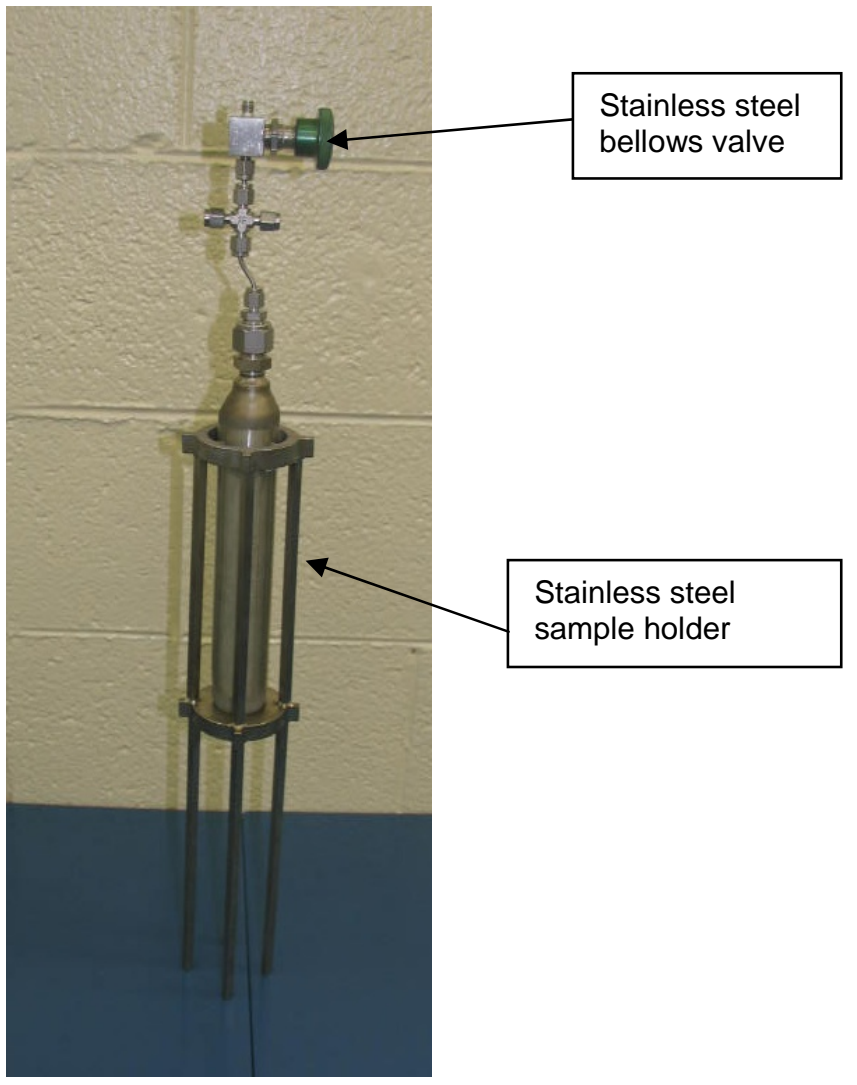

Fig. 5. HFIR sample container stand.

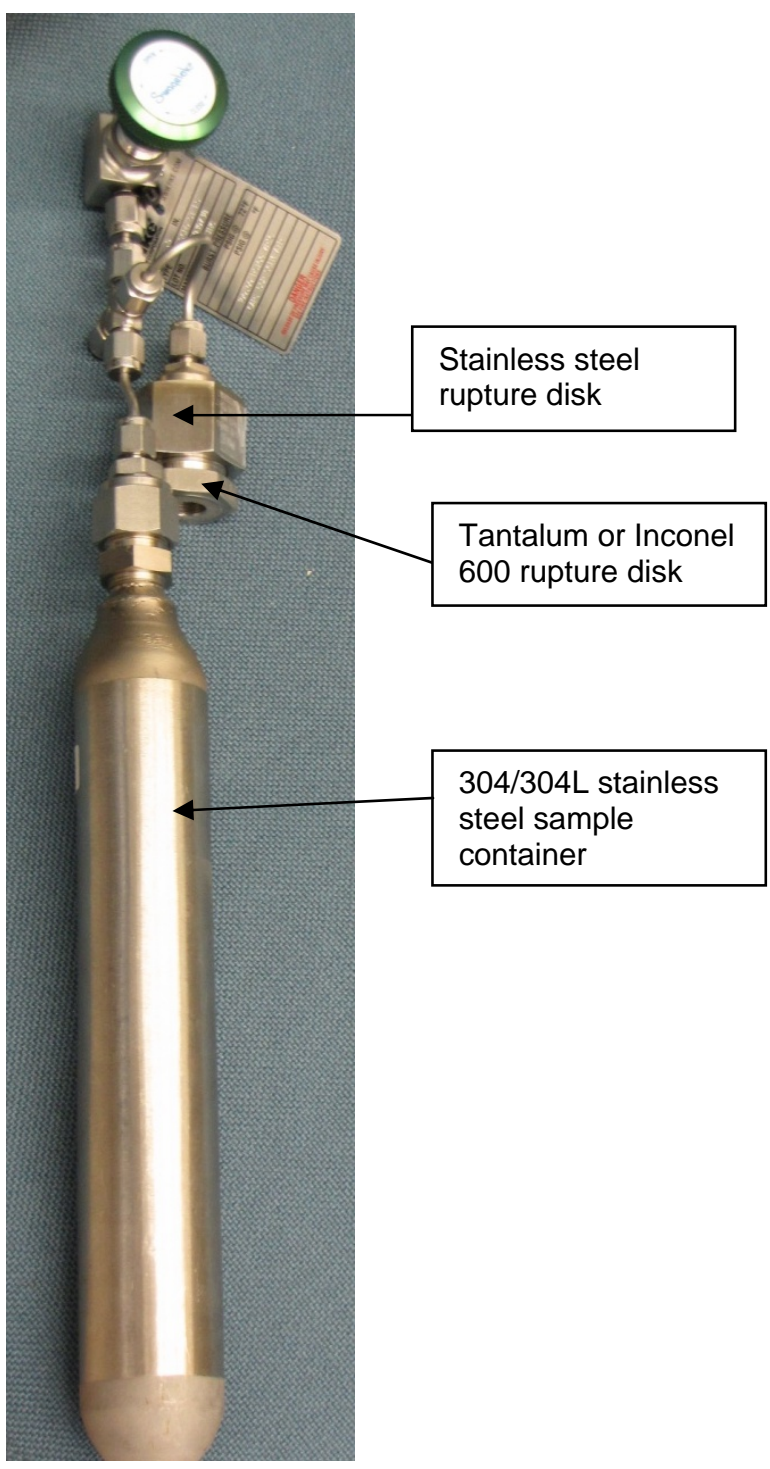

Fig. 6. HFIR sample container.

In preparation for irradiating the samples at HFIR, a sketch of the sample containers and configuration, a list of materials and quantities, the MSDS, purchase records, and fabrication drawings were provided in order to comply with HFIR documentation requirements.

Once the sample containers were loaded with resin and simulant and were ready to be irradiated, they were delivered to the irradiation facility at HFIR. Once there, the operators at the facility handled the samples. The irradiation facility determined the specific fuel element and provided the information used to determine the amount of time needed to acquire the correct dose. The irradiation time varied from $4 \mathrm{~h}$ to 5 days, depending on the dose. The temperature was monitored and ranged from $48^{\circ} \mathrm{C}$ to $51^{\circ} \mathrm{C}$.

After the irradiation, the samples were returned and were connected to the gas-sampling manifold used in the Co-60 source experiments. The gas and liquid samples were obtained and delivered to the Y-12 Analytical Laboratory, where they were tested for VOCs and SVOCs. The resin was also characterized based on visual observations. After the characterizations, $K_{d}$ tests were performed (in triplicate) on the resin with the Hanford simulant, found in Sect. 4.2. The sample canister is shown being lowered into the HFIR spent fuel pool in Fig. 7 and into a spent fuel element in Fig. 8. 


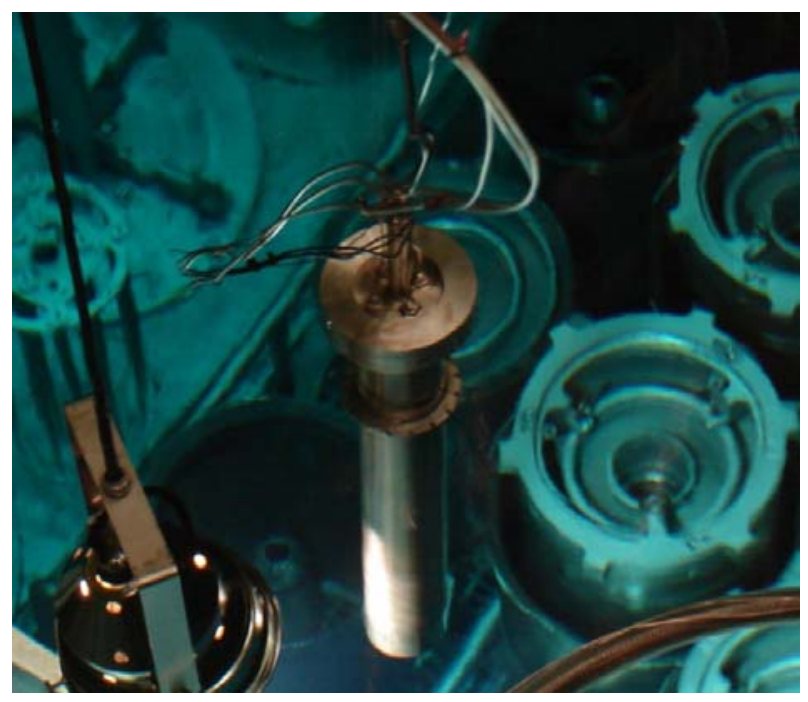

Fig. 7. Sample canister being lowered into the HFIR spent fuel pool.

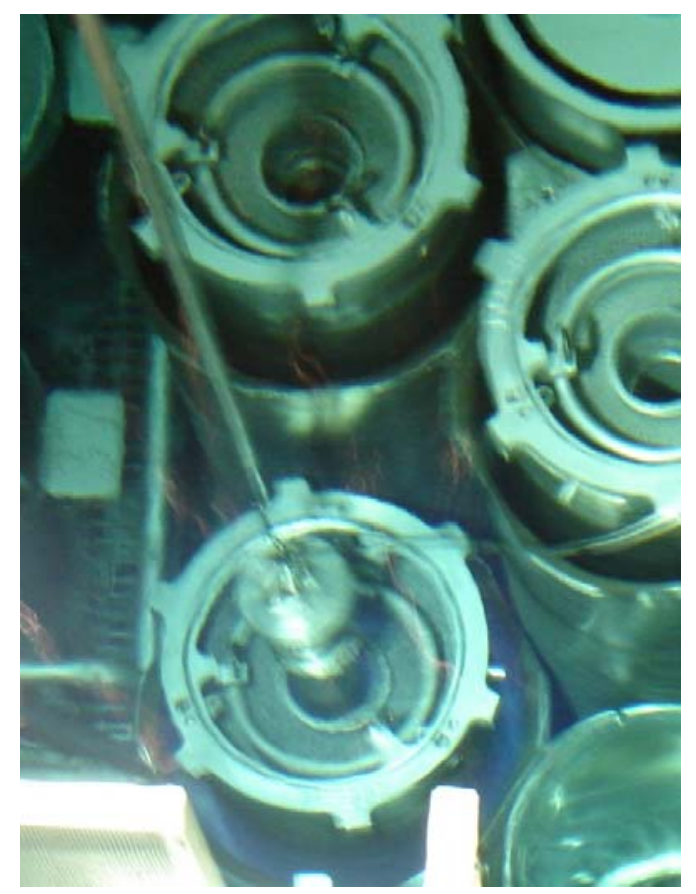

Fig. 8. Sample canister being lowered into a spent fuel element.

\subsubsection{Gas volume determinations}

Gas volumes were measured using a manifold configuration consisting of a sampling port for the gas sample to expand into, a vacuum pump, a pressure transducer, a sample vessel connector, and a $2 \mathrm{~L}$ metering chamber (Fig. 1).

The sample container gas headspace volume in the large-volume sample containers needed to be determined. Before the large samples were sent to SRS for irradiation up to $100 \mathrm{Mrad}$, the gas volume for each sample was determined using a calibrated baratron pressure transducer, a 2-L expansion vessel, and a vacuum pump. The baseline pressure of the evacuated, 2-L expansion vessel was recorded. The 2-L expansion vessel was then isolated from the rest of the system. The sample chamber with the resin and solution was connected to the system. With the exception of the 2-L vessel, air from the room was permitted throughout the piping system.

After the system was closed to the room air, the pressure of the system was measured and recorded. The air in the closed system was permitted to expand into the 2-L vessel, and the new, lower pressure was recorded. The pressure of the empty 2-L vessel and the two system pressures were used to determine the internal gas-phase volume of the entire system. The sample chamber was closed to the system, and the internal volume of the system with the sample chamber and the 2-L vessel were determined in a similar fashion. The sample chamber was opened to the room air one final time before its valve was closed and capped. The same procedure was used to determine the internal gas volume for the other two samples before their sample chambers were sealed. The sealed sample chambers were then sent to SRNL. The manifold, headspace for the HFIR samples, and the gas grab sample bottle volumes were also determined in this way.

The final volumes can be found in Table 2. The difference in the manifold volume between the Co- 60 source and the HFIR samples is due to the connector used to connect to the sample container (quick connect for the Co-60 source and a screwed connector for the HFIR samples). 
Table 2. Gas volumes for radiolysis testing

\begin{tabular}{lc}
\hline & Volume $\left(\mathbf{c m}^{\mathbf{3}}\right)$ \\
\hline Manifold—Co-60 source & 87.7 \\
Manifold—HFIR samples & 85.5 \\
Co-60 source sample void space—- $\mathrm{HNO}_{3}$ & 620.7 \\
Co-60 source sample void space—-water & 618.3 \\
Co-60 source sample void space—SRS & 618.7 \\
HFIR sample containers void space & 35 \\
Gas sample & 10 \\
\hline
\end{tabular}

\subsubsection{Gas Sampling}

Gas samples were collected using sample bottles supplied by the Analytical Services organization at the Y-12 National Security Complex. The sample bottles used were $10 \mathrm{~mL}$ in volume and were sealed with valves at their upper and lower ends. Both a sample bottle and the appropriate irradiation vessel were connected to the gas manifold (Fig. 1). With the sampling bottle open to the manifold and the sample source closed, the manifold and sample bottle were evacuated, and the pressure was recorded. The level of vacuum was approximately 0.2 Torr. The sample bottle valve was closed, and the vacuum pump was turned off and isolated from the manifold. The sample source was opened to the manifold, allowing gas to transfer into the manifold. The pressure in the manifold was recorded and was used to calculate the sample volume. The sample bottle was then opened to the manifold, allowing collection of a sample for analysis. The pressure was measured in this configuration to facilitate determination of the sample volume sent for analysis.

\subsubsection{Radiolysis Sample Analysis}

Gas compositions were determined using a Varian Aerograph gas chromatograph equipped with Porapak and molecular sieve columns and a photoionization detector (PID). The gas was also analyzed by mass spectrometry, with a VG Micromass Ltd. VG 3001.

The SVOCs in the liquid samples were analyzed using a Hewlett Packard Model 5989A gas chromatograph/mass spectrometer (GC/MS).

The total organic carbon was analyzed using a Tekmar-Dohrmann DC-80 total organic carbon analyzer.

\subsection{CESIUM PARTITIONING TEST ( $\mathrm{K}_{\mathrm{d}}$ DETERMINATIONS)}

Partitioning testing was performed to obtain equilibrium isotherm data for spherical RF resin for incorporation into a column performance prediction model, which will be utilized in the design of equipment for remediation of stored Hanford and/or SRS waste. Previous determinations have been made and used to develop two isotherm predictors. The work reported here obtained additional data over a broader range of cesium and hydroxide concentrations, which is to be used to improve existing models. The tests performed were also intended to evaluate the stability of the resin over extended periods of contact with tank waste and the effect of radiation exposure on resin performance.

Prior to each set of equilibrations, volumes of resin adequate for both equilibration tests and F-factor determinations were collected from the pretreated resin that was stored in the desired state (Na- or $\mathrm{H}$-form) under liquid and inert gas (argon).

For the method validation tests, two samples were removed from the pretreated (Na form) resin for determination of a conversion factor between damp and dry Na-form resin masses (F-factors, see Sect. 2.1.2). Volumes of resin were removed from the supply container by submerging the larger-diameter 
end of a glass pipette into the supply container, allowing resin slurry to fill the end of the tube, then sealing the other end of the tube to prevent venting. The sample material in the tube was transferred to a Buchner funnel into which a Whatman 41 filter disc had been placed. The filter was prewetted with DI water to seal the filter paper to the funnel. Vacuum was initiated after wetting the filter and prior to sample transfer. In each instance, free water was removed by maintaining vacuum until transfer of water from the filter into the catch flask was no longer observed. Vacuum was maintained for 2 min after the last drop of free liquid was observed to fall from the filter. Additional resin samples for use in equilibration testing were collected during the procedure in which F-factor samples were prepared. In the case of the method validation test, water removal was performed under normal atmosphere (i.e., not under inert gas). In all subsequent $\mathrm{K}_{\mathrm{d}}$ determination tests, the resin was prepared as described in Sect. 2.1 of this report; per that description, all $\mathrm{K}_{\mathrm{d}}$ determinations other than the initial method validation tests were performed using $\mathrm{H}$-form resin. The decision to perform $\mathrm{Cs}$ loading determinations based on $\mathrm{H}$-form resin was based on the variability in Na loading on prepared sodium-form resin, due to the potential exchange of $\mathrm{H}^{+}$for $\mathrm{Na}^{+}$during water washing of the sodium-form material prior to its use.

\subsection{TITRATIONS}

The objective of the titration evaluation was to obtain sodium uptake data corresponding to the conversion of RF resin from hydrogen to sodium form, in order to support modeling of RF column performance. The tests performed were designed to examine the effects of sodium ion concentration, free hydroxide concentration, and choice of counter ion on conversion from hydrogen to sodium form.

Previous titration determinations have been performed, and the data were used to develop predictors of resin performance. Analogous to the $\mathrm{K}_{\mathrm{d}}$ determinations reported in this document, additional titrations were performed over expanded titrant composition ranges to obtain data for model enhancement.

Testing was performed in three phases. The objective of the first phase was to identify an appropriate background salt for use in high ionic strength testing. The objective of the second phase was to establish a titration curve at lower sodium concentrations using a titrant in which sodium is present in only $\mathrm{NaOH}$ form. The third phase of testing focused on the effects of increased sodium concentration in the titrant on cation exchange. Due to the anticipated existence of inflection points in the titration curve at lower sodium concentrations, testing was performed at several initial $\mathrm{pH}$ values to isolate these points. After being dewatered, damp resin samples were transferred to polymer flasks (typically polypropylene) containing the desired titrant. The slurries produced were purged with argon, sealed, and transferred to a temperature-controlled orbital shaker. The equilibration temperature was controlled in the range $25^{\circ} \mathrm{C}$ to $26^{\circ} \mathrm{C}$ during all testing. The agitation rate was maintained at approximately $125 \mathrm{rpm}$.

An autotitrator was used to measure the hydroxide concentration of the samples. After contact, a portion of the titrant solution was placed into a glass beaker with a stir bar. The autotitrator has a $\mathrm{pH}$ probe (to be placed in the beaker) and a tube that dispenses the acid or base that is needed for the titration. $0.01 M$ hydrochloric acid was used as the titration solution. The titrator will also let the user choose a set point for the titration, which was 7.0 in all cases.

\section{MATERIALS}

\subsection{DISSOLVED SALT SURROGATE COMPOSITIONS}

Table 3 shows the recipes for making the SRS and Hanford simulants. The SRS simulant recipe was the average composition of Tanks 1, 2, and 3 (Smith 2007). The Hanford simulant recipe was found in the literature also (Arm et al. 2006). 
Table 3. Compositions of dissolved salt surrogates

\begin{tabular}{ccc}
\hline $\begin{array}{c}\text { Chemical } \\
\text { compound }\end{array}$ & $\begin{array}{c}\text { SRS Tanks 1, 2, and 3 } \\
\text { (g/L) }\end{array}$ & $\begin{array}{c}\text { Hanford Tank AP-101 } \\
\text { (g/L) }\end{array}$ \\
\hline $\mathrm{NaNO}_{3}$ & 336.7 & 154.2 \\
$\mathrm{NaNO}_{2}$ & 16.6 & 49.0 \\
$\mathrm{NaOH}$ & 58.4 & 81.6 \\
$\mathrm{NaAlO}_{2}$ & 10.7 & 21.3 \\
$\mathrm{Na}_{2} \mathrm{CO}_{3}$ & 8.5 & 47.7 \\
$\mathrm{Na}_{2} \mathrm{SO}_{4}$ & 4.3 & 5.7 \\
$\mathrm{NaCl}$ & 0.82 & 2.3 \\
$\mathrm{NaF}$ & 1.2 & 0.0 \\
\hline
\end{tabular}

Cesium nitrate and potassium nitrate that are normally in the simulants were replaced with additional sodium nitrate to prevent cesium and potassium uptake by the RF resin, which could impact the $\mathrm{K}_{\mathrm{d}}$ tests performed later. If cesium was needed, it was added as cesium nitrate. Also, minor components such as chromium and phosphate were not included.

\section{RESULTS}

\subsection{RADIOLYSIS}

\subsubsection{Analytical Results-Gas}

The gas was sampled using the gas sampling procedure in Sect. 2.2.4. The pressure was recorded and used to determine the amount of gas produced. The samples were analyzed at the Y-12 Analytical Laboratory for hydrogen, oxygen, nitrogen, nitrous oxide, carbon monoxide, carbon dioxide, and VOCs. The number of moles calculated was based on gas pressure measurements. The volume fractions were used to determine the number of moles produced for each gas, as seen in Table 4. The analyzed gases do not add up to the total volume of gas produced. The HFIR sample gas analysis is for the whole accumulated dose, where the Co-60 source samples were vented after each sample was taken. The raw data obtained can be found in Appendix A.

As can be seen in Table 5, hydrogen is the predominant generated gas species, and a small amount of methane is also produced. The gas generation data in Table 5 are only for $50 \mathrm{Mrad}$ increments. The sample containers were vented after every gas sample was taken. The total amount of gas also seems to increase as the dose rate increases. When the initial volume of air in the canister was subtracted from the amount of gas generated, some values were negative, which indicates an error in the analytical results or that the gas was consumed. The samples with the negative number of moles generated were assumed to have zero number of moles of that specific gas produced, except for oxygen. Oxygen can be consumed by the resin, so those negative values that are shown can represent either consumed oxygen or analytical error.

Gas analysis results for the Co-60 source samples can be found in Table 5. The original gas grab sample containers for analytical purposes were made of glass with Teflon stopcocks, which were found to leak over time. These sample containers were used only with the 150 Mrad samples; Y-12 provided the stainless steel gas sample containers for the $175 \mathrm{Mrad}$ to $300 \mathrm{Mrad}$ samples and for the HFIR samples. The pressure readings were still valid, so the total number of moles of gas could be calculated, but there were no analytical results for the sample irradiated to $150 \mathrm{Mrad}$. 
Table 4. Gas produced during HFIR sample radiolysis ${ }^{a}$

\begin{tabular}{|c|c|c|c|c|c|c|c|c|}
\hline \multicolumn{2}{|c|}{ HFIR samples } & \multicolumn{5}{|c|}{ Number of moles generated per gram dry resin } & \multicolumn{2}{|c|}{$\begin{array}{l}\text { Number of moles generated per } \\
\text { gram dry resin per Mrad }\end{array}$} \\
\hline & Dose (Mrad) & Total & Hydrogen & Methane & Nitrogen & Oxygen & Total & Hydrogen \\
\hline \multirow[t]{4}{*}{ Nitric acid } & 300 & $2.0774 \mathrm{E}-04$ & $3.4734 \mathrm{E}-05$ & $2.0657 \mathrm{E}-08$ & $4.7800 \mathrm{E}-05$ & $-5.4401 \mathrm{E}-06$ & $6.9247 \mathrm{E}-07$ & $1.1578 \mathrm{E}-07$ \\
\hline & 250 & $8.9612 \mathrm{E}-04$ & $6.9001 \mathrm{E}-05$ & $8.9443 \mathrm{E}-08$ & $1.2685 \mathrm{E}-04$ & $-1.9257 \mathrm{E}-05$ & $3.5845 \mathrm{E}-06$ & $2.7601 \mathrm{E}-07$ \\
\hline & 200 & $1.4560 \mathrm{E}-03$ & $3.6152 \mathrm{E}-04$ & $2.9103 \mathrm{E}-07$ & $3.5762 \mathrm{E}-04$ & $-1.9358 \mathrm{E}-05$ & $7.2800 \mathrm{E}-06$ & $1.8076 \mathrm{E}-06$ \\
\hline & 150 & $7.7084 \mathrm{E}-05$ & $5.0258 \mathrm{E}-06$ & $1.5248 \mathrm{E}-08$ & & $-1.2742 \mathrm{E}-05$ & $5.1389 \mathrm{E}-07$ & $3.3505 \mathrm{E}-08$ \\
\hline \multirow[t]{8}{*}{ Water } & 300 & $3.5083 \mathrm{E}-04$ & $2.8347 \mathrm{E}-04$ & $1.4019 \mathrm{E}-07$ & & $-1.4957 \mathrm{E}-05$ & $1.1694 \mathrm{E}-06$ & $9.4490 \mathrm{E}-07$ \\
\hline & 250 & $1.6364 \mathrm{E}-04$ & $9.6861 \mathrm{E}-05$ & $9.8041 \mathrm{E}-08$ & & $-1.6948 \mathrm{E}-05$ & $6.5458 \mathrm{E}-07$ & $3.8744 \mathrm{E}-07$ \\
\hline & 200 & $1.7506 \mathrm{E}-04$ & $1.7461 \mathrm{E}-08$ & $1.2611 \mathrm{E}-04$ & & $3.1027 \mathrm{E}-05$ & $8.7530 \mathrm{E}-07$ & $8.7306 \mathrm{E}-11$ \\
\hline & 150 & $1.8119 \mathrm{E}-04$ & $1.0237 \mathrm{E}-04$ & $1.0857 \mathrm{E}-07$ & & $-1.7044 \mathrm{E}-05$ & $1.2079 \mathrm{E}-06$ & $6.8247 \mathrm{E}-07$ \\
\hline & $100(\text { new })^{b}$ & $1.7922 \mathrm{E}-04$ & $2.0251 \mathrm{E}-06$ & $1.7776 \mathrm{E}-08$ & $7.8672 \mathrm{E}-05$ & $1.5913 \mathrm{E}-05$ & $1.7922 \mathrm{E}-06$ & $2.0251 \mathrm{E}-08$ \\
\hline & $50(\text { new })^{b}$ & & & & & & & \\
\hline & $100(\text { old })^{b}$ & $1.7292 \mathrm{E}-04$ & $2.3794 \mathrm{E}-05$ & $1.7146 \mathrm{E}-08$ & $3.7984 \mathrm{E}-05$ & $2.2761 \mathrm{E}-05$ & $1.7292 \mathrm{E}-06$ & $2.3794 \mathrm{E}-07$ \\
\hline & $50(\text { old })^{b}$ & $9.1415 \mathrm{E}-05$ & $9.0967 \mathrm{E}-09$ & $9.1269 \mathrm{E}-08$ & $7.0933 \mathrm{E}-06$ & $2.6465 \mathrm{E}-06$ & $1.8283 \mathrm{E}-06$ & $1.8193 \mathrm{E}-10$ \\
\hline \multirow[t]{4}{*}{ Hanford } & 300 & $1.2755 \mathrm{E}-04$ & $7.3035 \mathrm{E}-05$ & $1.2609 \mathrm{E}-08$ & & $-1.7050 \mathrm{E}-05$ & $4.2517 \mathrm{E}-07$ & $1.4790 \mathrm{E}-07$ \\
\hline & 250 & $2.2244 \mathrm{E}-04$ & $1.5026 \mathrm{E}-04$ & $4.4343 \mathrm{E}-08$ & & $-1.5372 \mathrm{E}-05$ & $8.8978 \mathrm{E}-07$ & $2.0872 \mathrm{E}-07$ \\
\hline & 200 & $1.5068 \mathrm{E}-04$ & $9.0405 \mathrm{E}-05$ & $2.9989 \mathrm{E}-08$ & & $-1.6867 \mathrm{E}-05$ & $7.5338 \mathrm{E}-07$ & $1.6470 \mathrm{E}-07$ \\
\hline & 150 & $1.7007 \mathrm{E}-04$ & $7.4846 \mathrm{E}-05$ & $1.2147 \mathrm{E}-09$ & & $1.6560 \mathrm{E}-05$ & $1.1338 \mathrm{E}-06$ & $1.2577 \mathrm{E}-07$ \\
\hline \multirow[t]{4}{*}{ SRS } & 300 & $3.8331 \mathrm{E}-04$ & $1.8138 \mathrm{E}-04$ & 7.6517E-08 & & $7.0678 \mathrm{E}-05$ & $1.2777 \mathrm{E}-06$ & $1.6442 \mathrm{E}-07$ \\
\hline & 250 & $3.0424 \mathrm{E}-04$ & $9.9366 \mathrm{E}-05$ & $6.0703 \mathrm{E}-08$ & & $6.3684 \mathrm{E}-05$ & $1.2170 \mathrm{E}-06$ & $1.0862 \mathrm{E}-07$ \\
\hline & 200 & $5.1334 \mathrm{E}-05$ & $2.1555 \mathrm{E}-05$ & 4.9875E-09 & & $-4.6398 \mathrm{E}-06$ & $2.5667 \mathrm{E}-07$ & $6.9042 \mathrm{E}-08$ \\
\hline & 150 & $1.8110 \mathrm{E}-04$ & $6.3024 \mathrm{E}-05$ & $1.4342 \mathrm{E}-08$ & & 4.4784E-05 & $1.2074 \mathrm{E}-06$ & $1.0142 \mathrm{E}-07$ \\
\hline
\end{tabular}

${ }^{a}$ Data in this table represent cumulative gas production. The water sample with new resin that was irradiated to 50 Mrad did not have a gas sample analyzed because the gas sample was accidentally evacuated.

${ }^{b}$ New resin is virgin resin and has no known oxygen exposure. The 'old' resin was used in the hydraulic conductivity test loop and has a known oxygen exposure of $7 \mathrm{mmoles}$ oxygen/g dry resin. 
Table 5. Gas produced during Co-60 source sample radiolysis ${ }^{a}$

\begin{tabular}{|c|c|c|c|c|c|c|c|c|}
\hline \multicolumn{2}{|c|}{ Co-60 source } & \multicolumn{5}{|c|}{ Number of moles generated per gram dry resin } & \multicolumn{2}{|c|}{$\begin{array}{l}\text { Number of moles generated per } \\
\text { gram dry resin per Mrad }\end{array}$} \\
\hline & Dose (Mrad) & Total & Total & Methane & Nitrogen & Oxygen & Total & Hydrogen \\
\hline \multirow[t]{5}{*}{ Nitric acid } & 300 & & & $1.3069 \mathrm{E}-08$ & $3.6302 \mathrm{E}-06$ & $-9.4903 \mathrm{E}-06$ & $2.6307 \mathrm{E}-06$ & $7.7028 \mathrm{E}-07$ \\
\hline & 250 & $8.9537 \mathrm{E}-05$ & $1.5400 \mathrm{E}-05$ & $6.1833 \mathrm{E}-09$ & $-7.9566 \mathrm{E}-06$ & $-9.5989 \mathrm{E}-06$ & $1.7907 \mathrm{E}-06$ & $3.0801 \mathrm{E}-07$ \\
\hline & 200 & $4.7218 \mathrm{E}-05$ & $2.4364 \mathrm{E}-06$ & $4.6376 \mathrm{E}-09$ & $-1.5470 \mathrm{E}-05$ & $-6.4116 \mathrm{E}-06$ & $9.4437 \mathrm{E}-07$ & $4.8729 \mathrm{E}-08$ \\
\hline & 175 & $6.0486 \mathrm{E}-05$ & & & $1.0730 \mathrm{E}-05$ & $2.4805 \mathrm{E}-06$ & $1.2097 \mathrm{E}-06$ & $0.0000 \mathrm{E}+00$ \\
\hline & 150 & $3.4314 \mathrm{E}-05$ & & & & & $6.8628 \mathrm{E}-07$ & $0.0000 \mathrm{E}+00$ \\
\hline \multirow[t]{2}{*}{ Water } & 300 & $5.5560 \mathrm{E}-05$ & $4.6948 \mathrm{E}-05$ & $3.2311 \mathrm{E}-09$ & $-3.7779 \mathrm{E}-05$ & $-1.1084 \mathrm{E}-05$ & $1.1112 \mathrm{E}-06$ & $9.3896 \mathrm{E}-07$ \\
\hline & 250 & $4.3365 \mathrm{E}-05$ & $3.1479 \mathrm{E}-05$ & $2.9330 \mathrm{E}-09$ & $-3.4662 \mathrm{E}-05$ & $-1.1571 \mathrm{E}-05$ & $8.6730 \mathrm{E}-07$ & $6.2957 \mathrm{E}-07$ \\
\hline \multirow[t]{5}{*}{ SRS } & 300 & $-3.0547 \mathrm{E}-06$ & $-5.0742 \mathrm{E}-07$ & $-7.1380 \mathrm{E}-10$ & $-4.6661 \mathrm{E}-05$ & $-1.2494 \mathrm{E}-05$ & $-6.1094 \mathrm{E}-08$ & $-1.0148 \mathrm{E}-08$ \\
\hline & 250 & $-2.2209 \mathrm{E}-05$ & $-3.3602 \mathrm{E}-06$ & $-4.5447 \mathrm{E}-09$ & $-5.8903 \mathrm{E}-05$ & $-1.5116 \mathrm{E}-05$ & $-4.4418 \mathrm{E}-07$ & $-6.7205 \mathrm{E}-08$ \\
\hline & 200 & $-2.6359 \mathrm{E}-06$ & $-1.9904 \mathrm{E}-07$ & $-3.6645 \mathrm{E}-10$ & $-4.6258 \mathrm{E}-05$ & $-1.2530 \mathrm{E}-05$ & $-5.2718 \mathrm{E}-08$ & $-3.9809 \mathrm{E}-09$ \\
\hline & 175 & $2.5376 \mathrm{E}-06$ & $3.5269 \mathrm{E}-07$ & $-8.5095 \mathrm{E}-11$ & $-4.3864 \mathrm{E}-05$ & $-1.1625 \mathrm{E}-05$ & $5.0752 \mathrm{E}-08$ & $7.0538 \mathrm{E}-09$ \\
\hline & 150 & $2.8171 \mathrm{E}-05$ & & & & & $5.6342 \mathrm{E}-07$ & $0.0000 \mathrm{E}+00$ \\
\hline
\end{tabular}

\footnotetext{
${ }^{a}$ Data in this table represent gas produced during each 50 Mrad sample.
} 
The stainless steel Co-60 source sample container for RF resin in water had a leaking weld that was not evident until collection of the $175 \mathrm{Mrad}$ gas grab. The resin and water were removed from the container, and it was re-welded and placed back into the irradiator when the other samples reached $200 \mathrm{Mrad}$. This explains why there is no gas data until $250 \mathrm{Mrad}$. It can also be noted that for the higher doses, the SRS simulant consumes more gas than is produced. It seems that every sample either consumed oxygen or that there is an analytical error.

\subsubsection{Analytical Results—Liquid}

In addition to gas sample analyses, solutions used in radiolysis tests were also analyzed for SVOCs by Y-12 Analytical Services. No standard SVOCs were found with GC/MS; however, the 2-pentanone, 4-hydroxy-4-methyl compound was flagged on nearly every sample. We are unsure if this compound is a degradation product or if there is an unknown compound that has the same GC-MS profile. The raw data from the SVOC tests are presented in Appendix B.

The Co-60 source liquid samples were also tested for total organic carbon at the Y-12 Analytical Laboratory and for formaldehyde at TestAmerica Laboratories (Nashville, Tennessee). The latter analysis did not detect any formaldehyde in the nitric acid or in the SRS simulant samples but detected $61.4 \mu \mathrm{g} / \mathrm{L}$ in the water sample. The raw data from these analyses can be found in Appendix B.

The total organic carbon analysis results can be found in Table 6. Five different aliquots were tested and all results are reported with the average for the liquid. The raw data can be found in Appendix B.

Table 6. Total organic carbon analytical results $(\mathrm{mg} / \mathrm{L})$

\begin{tabular}{lcccccc}
\hline \multicolumn{1}{c}{ Liquid } & \multicolumn{7}{c}{ Individual aliquots } & Average \\
\hline Nitric acid & 27.2 & 27.31 & 26.61 & 28.3 & 26.65 & 27.21 \\
Water & 108 & 104.4 & 104.7 & 110.9 & 112.9 & 108.18 \\
SRS & 1760 & 1711 & 1754 & 1782 & 1784 & 1758.2 \\
\hline
\end{tabular}

\section{2 $\quad \mathrm{K}_{\mathrm{d}}$ DETERMINATIONS}

\subsubsection{Method Validation Testing}

The objectives of validation testing were to demonstrate a procedure for determining reproducible cesium distribution results, to develop familiarity with the method, and to isolate procedural variables that may affect the ability to obtain accurate, reproducible results. To limit the number of variables present in testing, a simple simulant consisting of a combination of sodium hydroxide and sodium chloride at concentrations of $2 M$ and $3 M$, respectively, was used. Stable cesium was added to the simulant at a nominal concentration of $4.95 \times 10^{-5} \mathrm{M}$.

Sodium-form resin samples used in equilibrations and F-factor determinations were collected as described previously in Sect. 2.1.2. F-factor samples having damp masses of approximately $0.1 \mathrm{~g}$ each were placed into ceramic specimen containers for differential thermal analysis and thermogravimetric analysis (DTA/TGA). Containers were tared before receipt of resin samples. Samples were weighed again immediately prior to DTA/TGA, and were weighed intermittently during analysis to determine the point at which no additional mass loss was occurring. The first RF sample was placed in the DTA/TGA a few minutes after the sample was prepared. The sample was heated to $95^{\circ} \mathrm{C}$ in flowing nitrogen until a constant weight was achieved. The weight loss was $59.2 \mathrm{wt} \%$. The second RF sample was prepared the following afternoon and stored overnight in a sealed plastic bottle. The sample was heated at $59^{\circ} \mathrm{C}$ in flowing nitrogen. The weight loss was $47.0 \mathrm{wt} \%$. This sample was then heated to $92^{\circ} \mathrm{C}$ in flowing nitrogen. The total weight loss increased to $50.7 \mathrm{wt} \%$. 
Equilibration tests were performed at an approximate resin-to-simulant ratio of $1 \mathrm{~g}$ dry resin: $100 \mathrm{~mL}$ simulant. Transfer of resin into equilibration flasks was accomplished by sluicing resin from sample vials into flasks using premeasured volumes of simulant (Table 7).

Table 7. Values recorded during preparation of first equilibration samples (g)

\begin{tabular}{cccccc}
\hline Flask No. & Empty mass & $\begin{array}{c}\text { Mass w/ } \\
\text { simulant }\end{array}$ & $\begin{array}{c}\text { Mass w/simulant } \\
\text { and resin }\end{array}$ & $\begin{array}{c}\text { Net mass } \\
\text { simulant }^{2}\end{array}$ & $\begin{array}{c}\text { Net mass } \\
\text { resin }^{a}\end{array}$ \\
\hline 1 & 90.81 & 207.76 & 209.48 & 116.95 & 1.72 \\
2 & 91.68 & 208.57 & 210.86 & 116.89 & 2.29 \\
3 & 92.59 & 209.62 & 211.84 & 117.03 & 2.22 \\
\hline
\end{tabular}

${ }^{a}$ Sodium form resin

The flasks were placed onto an orbital shaker equipped with a temperature-controlled enclosure. The enclosure temperature set point was adjusted to $25^{\circ} \mathrm{C}$. The enclosure temperature at the time the flasks were placed onto the shaker table was $25.3^{\circ} \mathrm{C}$. The shaker table was set at an agitation speed of $150 \mathrm{rpm}$ after determining that this rate provided thorough agitation of the resin without leaving residual resin on the flask wall above the liquid surface. The enclosure temperature was recorded at least twice daily. The equilibration temperature remained relatively constant at $25.3^{\circ} \mathrm{C}$ for the first $36 \mathrm{~h}$ of testing. When observed at $48 \mathrm{~h}$, the equilibration temperature had increased to $25.5^{\circ} \mathrm{C}$, at which point the shaker table temperature set point was reset to $24.7^{\circ} \mathrm{C}$. However, the enclosure temperature remained at $25.5^{\circ} \mathrm{C}$ to $25.6^{\circ} \mathrm{C}$ throughout the remainder of the equilibration. Agitation was terminated after $72 \mathrm{~h}$.

Equilibrations performed in this particular phase of testing were not performed under inert atmosphere.

Samples of the liquids (supernatants) were collected after allowing the resin beads to settle. Samples having a volume of 5-mL were pipetted into HDPE sample bottles. After collecting liquid samples the contents of each equilibration flask were transferred to a Buchner funnel for recovery of resin. Resin was collected in a manner similar to that used for dewatering the resin for water retention determinations; i.e., the resin was vacuum filtered under normal atmosphere with vacuum being maintained for approximately 2 min after the last drop of water was observed to fall from the funnel into the receiving flask. After dewatering, the resins samples were transferred into HDPE bottles. Sample bottle masses measured before and after transfer of samples are presented in Table 8. In addition to the samples listed above, a "blank" sample of the untreated, Cs-containing simple simulant was also collected.

Table 8. Masses of liquid and resin samples from method validation tests (g)

\begin{tabular}{clccc}
\hline Sample No. & Description & $\begin{array}{c}\text { Sample bottle } \\
\text { tare mass }\end{array}$ & Mass w/sample & $\begin{array}{c}\text { Net sample } \\
\text { mass }\end{array}$ \\
\hline R1 & Simulant & 12.520 & 18.325 & 5.805 \\
R2 & Simulant & 12.531 & 18.400 & 5.869 \\
R3 & Simulant & 12.536 & 18.380 & 5.844 \\
S1 & Resin & 12.528 & 14.413 & 1.885 \\
S2 & Resin & 12.537 & 14.780 & 2.243 \\
S3 & Resin & 12.590 & 14.889 & 2.299 \\
\hline
\end{tabular}

Resin samples were ashed and leached prior to being analyzed by inductively coupled plasma/mass spectrophotometry (ICP/MS). Simulant samples were analyzed by ICP/MS directly. Results are presented in Table 9. Distribution coefficients obtained were a factor of 2 to 3 less than predicted values using the most recent CERMOD isotherm. 
Table 9. Cesium uptake results from method validation test

\begin{tabular}{cccc}
\hline Equilibration & $\begin{array}{c}\text { Raffinate Cs } \\
\text { conc. } \\
(\boldsymbol{\mu g} / \mathbf{m L})\end{array}$ & $\begin{array}{c}\text { Measured } \\
\text { resin Cs conc., } \\
\mathbf{~ m m o l} / \mathbf{g} \text { resin }\end{array}$ & $\mathbf{D}_{\mathbf{C s}}{ }^{\boldsymbol{a}, \boldsymbol{b}}$ \\
\hline 1 & $1.36 \times 10^{-1}$ & $1.96 \times 10^{-3}$ & $1.92 \times 10^{3}$ \\
2 & $1.24 \times 10^{-1}$ & $1.69 \times 10^{-3}$ & $1.81 \times 10^{3}$ \\
3 & $1.34 \times 10^{-1}$ & $1.74 \times 10^{-3}$ & $1.72 \times 10^{3}$ \\
\hline \multicolumn{4}{c}{ Simulant consisting of $2 \mathrm{M} \mathrm{NaOH}, 3 \mathrm{M} \mathrm{NaCl}$, and containing stable } \\
cesium at a nominal concentration of $4.95 \times 10^{-5} \mathrm{M}$ was used. \\
${ }^{b}$ Values are based on resin mass in sodium form and on analysis of \\
digested resin, not on gamma counting.
\end{tabular}

\subsubsection{Second Equilibrations-Method Verification}

In light of the lower-than-expected distribution coefficients obtained in the method validation test, a decision was made to perform a second test using the same simulant as was used previously $(2 \mathrm{M} \mathrm{NaOH}$, $3 \mathrm{M} \mathrm{NaCl}$ in DI water) containing cesium at a nominal concentration of $4.95 \times 10^{-5} \mathrm{M}$ as before. To address uncertainty concerning detection limits for cesium analysis by ICP/MS, a trace quantity of Cs-137 was added to the simulant in the second set of equilibrations to facilitate determination of cesium concentrations by gamma counting. Unlike the previous tests, resin/simulant slurries were blanketed with argon before being sealed and transferred to the orbital shaker for equilibration to mitigate against resin oxidation.

The resin was pretreated according to the procedure described previously, Sect. 2.1.2, for hydrogenform material. Equilibrium distribution determinations were made at controlled temperatures of $25^{\circ} \mathrm{C}$ and $45^{\circ} \mathrm{C}$. (During testing, the equilibration temperatures during the nominal $25^{\circ} \mathrm{C}$ test were observed to vary between $25^{\circ} \mathrm{C}$ and $26^{\circ} \mathrm{C}$. During $45^{\circ} \mathrm{C}$ testing the actual temperature varied between $45^{\circ} \mathrm{C}$ and $45.5^{\circ} \mathrm{C}$.) As before, the resin-to-simulant ratio used in testing was $1 \mathrm{~g}$ resin: $100 \mathrm{~mL}$ simulant. Resin/simulant contacts at both temperatures were performed for periods of 48,72 , and $96 \mathrm{~h}$, and samples were analyzed to verify attainment of equilibrium. Duplicate tests were performed for each equilibration duration at both temperature conditions.

Equilibration results obtained were internally consistent; i.e., experimental values exhibited very low standard deviations (Table 10). The results were consistently lower than CERMOD predictions and previous experimental results obtained at SRNL by a factor of 2, as indicated in Fig. 9. In that regard, the results are consistent with results obtained in the previous test at ORNL.

\subsubsection{Third Equilibrations-Method Diagnosis}

Based on the results obtained to this point in the test program, it was speculated that the offset between ORNL experimental results and predicted values (based on previous SRNL results) could be due to differences in the location of the resin within the storage resin container as received from the manufacturer. Resin used at ORNL was taken from the top of the storage container and may have been more prone to oxidation than resin located deeper in the drum. It was decided that ORNL and SRNL would perform duplicate verification tests using resin and simulant that was prepared at SRNL in an attempt to isolate sources of the discrepancies between results from current experiments and the isotherms developed from prior data.

Resin preparation, F-factor determinations, and equilibrations were performed as in the immediately preceding test. Duplicate equilibrations using SRNL-provided materials were performed at $25^{\circ} \mathrm{C}$ and $45^{\circ} \mathrm{C}$ with contact times of 48 and $72 \mathrm{~h}$. Additional duplicate equilibrations were performed using ORNLprepared resin (same source as the previous test) at $25^{\circ} \mathrm{C}$ and $45^{\circ} \mathrm{C}$ with 72 -h contact times. 
Table 10. Results of second equilibrations (method verification)

\begin{tabular}{ccccc}
\hline $\begin{array}{c}\text { Contact time } \\
\mathbf{( h )}\end{array}$ & $\begin{array}{c}\text { Equilibration } \\
\text { temperature } \\
\left({ }^{\circ} \mathbf{C}\right)\end{array}$ & $\begin{array}{c}\text { Raffinate Cs } \\
\text { conc., } \\
\boldsymbol{M}\end{array}$ & $\begin{array}{c}\text { Resin Cs conc., } \\
\text { mmol/g resin }\end{array}$ & $\begin{array}{c}\text { Cs Distribution } \\
\text { coefficient, } \\
\mathbf{D}_{\mathbf{C s}} \boldsymbol{a} \boldsymbol{b}\end{array}$ \\
\hline 48 & 25 & $2.31 \mathrm{E}-06$ & $4.59 \mathrm{E}-03$ & 1984.8 \\
48 & 25 & $2.00 \mathrm{E}-06$ & $4.78 \mathrm{E}-03$ & 2387.0 \\
72 & 25 & $1.53 \mathrm{E}-06$ & $4.71 \mathrm{E}-03$ & 3082.2 \\
72 & 25 & $1.46 \mathrm{E}-06$ & $4.63 \mathrm{E}-03$ & 3177.5 \\
96 & 25 & $1.58 \mathrm{E}-06$ & $4.69 \mathrm{E}-03$ & 2969.0 \\
96 & 25 & $1.70 \mathrm{E}-06$ & $4.83 \mathrm{E}-03$ & 2833.6 \\
48 & 45 & $3.68 \mathrm{E}-06$ & $4.49 \mathrm{E}-03$ & 1222.1 \\
48 & 45 & $3.73 \mathrm{E}-06$ & $4.53 \mathrm{E}-03$ & 1214.4 \\
72 & 45 & $3.95 \mathrm{E}-06$ & $4.47 \mathrm{E}-03$ & 1131.0 \\
72 & 45 & $4.11 \mathrm{E}-06$ & $4.57 \mathrm{E}-03$ & 1113.2 \\
96 & 45 & $4.03 \mathrm{E}-06$ & $4.43 \mathrm{E}-03$ & 1098.9 \\
96 & 45 & $4.06 \mathrm{E}-06$ & $4.41 \mathrm{E}-03$ & 1084.4 \\
\hline
\end{tabular}

${ }^{a}$ Simulant consisting of $2 \mathrm{M} \mathrm{NaOH}, 3 \mathrm{M} \mathrm{NaCl}$, and containing stable cesium at a nominal concentration of $4.95 \times 10^{-5} \mathrm{M}$ was used.

${ }^{b}$ Values were determined based on analysis of initial and final cesium concentrations by gamma counting. Resin loadings were determined by mass balance. Resin was in hydrogen form, initially.

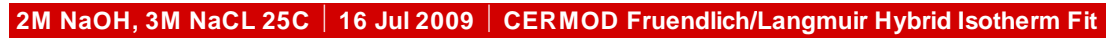

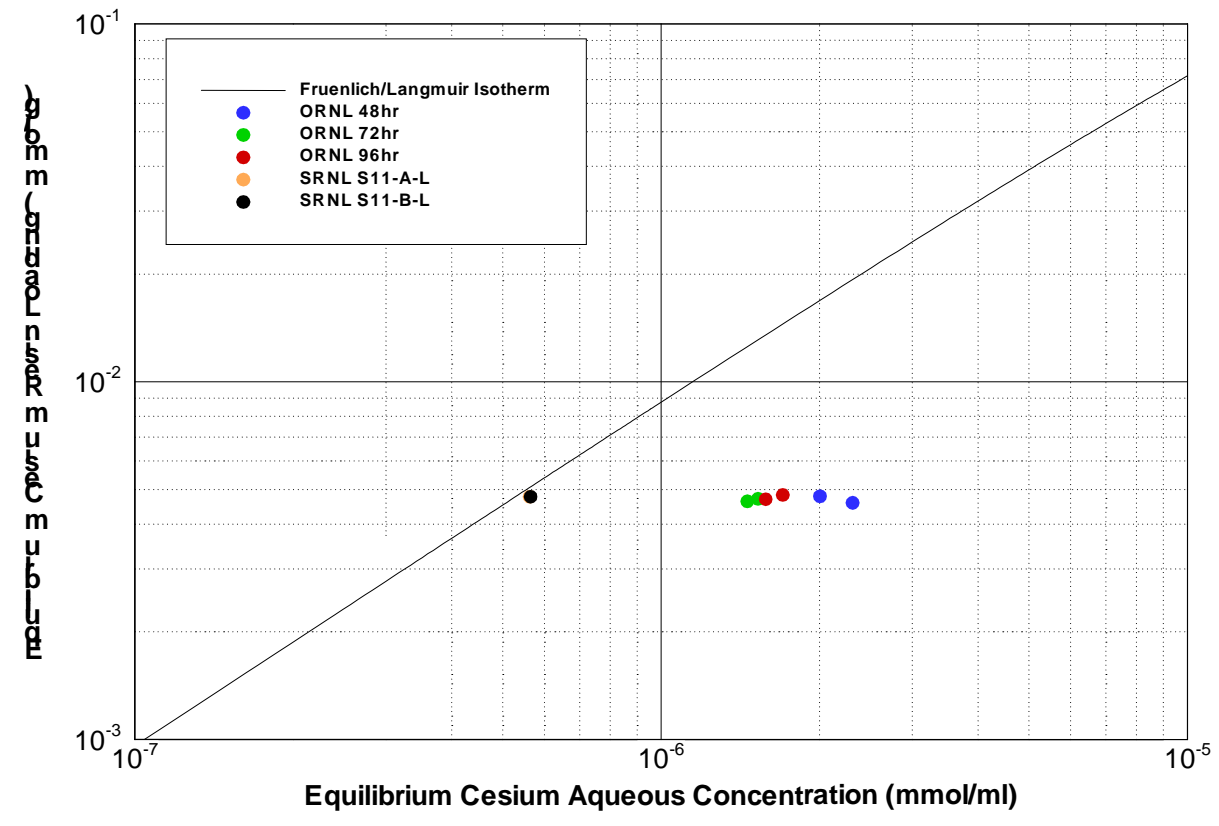

Fig. 9. Comparison of ORNL results from second equilibration test with prior data. (Figure provided by SRNL.) 
Samples at four randomly-selected conditions were also analyzed by ICP/MS. The initial cesium concentration used in this test was $4.95 \times 10^{-5} \mathrm{M}$. Simulant used in testing was provided by SRNL; its composition is given in Table 11.

Distribution coefficients obtained using SRNL-supplied materials are internally consistent, are lower than those obtained in previous tests at ORNL, and are 10\%-15\% less than the values obtained in the duplicate determinations using ORNL-prepared resin (Table 12). The reduction in cesium uptake in these tests relative to the previous two experiments can be attributed to the presence of potassium in the SRNLprovided simulant. The results are consistent with those obtained in the comparison tests performed at SRNL (Table 13). The hypothesized depression of $\mathrm{D}_{\mathrm{Cs}}$ values due to a less-favorable location of ORNLprepared resin within the storage drum is not supported by the test results, as distribution coefficients using this material are $10 \%$ to $15 \%$ higher than values obtained using SRNL-prepared resin. Results

Table 11. Composition of simulant used in method diagnosis test

\begin{tabular}{lcc}
\hline Component & Target conc., $\boldsymbol{M}$ & Measured conc., $\boldsymbol{M}$ \\
\hline $\mathrm{Na}$ & 6.00 & 6.26 \\
$\mathrm{~K}$ & 0.0070 & 0.0076 \\
$\mathrm{Rb}$ & 0.0063 & 0.0001 \\
$\mathrm{Free} \mathrm{OH}^{-}$ & 0.760 & 0.800 \\
$\mathrm{Al}$ & 0.259 & 0.319 \\
$\mathrm{NO}_{3}{ }^{-}$ & 4.190 & 4.935 \\
$\mathrm{NO}_{2}{ }^{-}$ & 0.149 & 0.171 \\
$\mathrm{Cl}^{-}$ & 0.003 & - \\
$\mathrm{F}^{-}$ & 0.003 & - \\
$\mathrm{PO}_{4}{ }^{3-}$ & 0.005 & - \\
$\mathrm{SO}_{4}{ }^{2-}$ & 0.032 & 0.033 \\
$\mathrm{P}$ & 0.005 & 0.005 \\
$\mathrm{~S}$ & 0.032 & 0.036 \\
\hline
\end{tabular}

Table 12. Results of third equilibrations (method diagnosis) ${ }^{a}$

\begin{tabular}{ccllc}
\hline $\begin{array}{c}\text { Contact time } \\
\text { (h) }\end{array}$ & $\begin{array}{c}\text { Equilibration } \\
\text { temperature } \\
\left({ }^{\circ} \mathbf{C}\right)\end{array}$ & $\begin{array}{l}\text { Resin } \\
\text { source }\end{array}$ & $\begin{array}{l}\text { Analysis } \\
\text { method }\end{array}$ & $\begin{array}{c}\text { Cesium } \\
\text { distribution }\end{array}$ \\
\hline 48 & 25 & SRNL & $\gamma$-counting & 1825.9 \\
48 & 25 & SRNL & $\gamma$-counting & 1894.5 \\
72 & 25 & SRNL & ICP/MS & 1423.8 \\
72 & 25 & SRNL & ICP/MS & 1628.9 \\
72 & 25 & SRNL & $\gamma$-counting & 1646.8 \\
72 & 25 & SRNL & $\gamma$-counting & 1718.0 \\
72 & 25 & ORNL & $\gamma$-counting & 2050.5 \\
72 & 25 & ORNL & $\gamma$-counting & 2003.8 \\
48 & 45 & SRNL & $\gamma$-counting & 948.9 \\
48 & 45 & SRNL & $\gamma$-counting & 937.4 \\
72 & 45 & SRNL & $\gamma$-counting & 906.0 \\
72 & 45 & SRNL & $\gamma$-counting & 854.5 \\
72 & 45 & SRNL & ICP/MS & 855.4 \\
72 & 45 & SRNL & ICP/MS & 990.4 \\
72 & 45 & ORNL & $\gamma$-counting & 1083.3 \\
72 & 45 & ORNL & $\gamma$-counting & 1093.1 \\
\hline
\end{tabular}

${ }^{a}$ Resin used was in hydrogen form. 
Table 13. Comparative results from equilibrations performed at SRNL

\begin{tabular}{ccccc}
\hline $\begin{array}{c}\text { Contact time } \\
\text { (h) }\end{array}$ & $\begin{array}{c}\text { Equilibration } \\
\text { temperature } \\
\left({ }^{\circ} \mathbf{C}\right)\end{array}$ & $\begin{array}{c}\text { Resin } \\
\text { source }\end{array}$ & $\begin{array}{c}\text { Analysis } \\
\text { method }\end{array}$ & $\begin{array}{c}\text { Cesium } \\
\text { distribution }\end{array}$ \\
\hline 48 & 25 & SRNL & $\gamma$-counting & 1710 \\
48 & 25 & SRNL & $\gamma$-counting & 1679 \\
72 & 25 & SRNL & $\gamma$-counting & 1563 \\
\hline
\end{tabular}

obtained using ICP/MS for Cs analysis are in close agreement with values obtained by gamma counting under identical test conditions. Results for F-factor determinations performed in conjunction with the equilibrations performed at ORNL are presented in Table 14 and Table 15.

Table 14. F-factor data for resin used in third equilibrations at $25^{\circ} \mathrm{C}$

\begin{tabular}{cccccc}
\hline $\begin{array}{c}\text { Empty vial } \\
\text { mass, } \mathbf{g}\end{array}$ & $\begin{array}{c}\text { Gross mass } \\
\text { w/damp resin, }\end{array}$ & $\begin{array}{c}\text { Gross mass w/ } \\
\text { dry resin, }\end{array}$ & $\begin{array}{c}\text { Damp resin } \\
\text { mass, } \mathbf{g}\end{array}$ & $\begin{array}{c}\text { Dry resin } \\
\text { mass, } \mathbf{g}\end{array}$ & F-factor $^{\boldsymbol{a}}$ \\
\hline 13.282 & 14.325 & 13.748 & 1.043 & 0.466 & 2.238 \\
13.269 & 14.386 & 13.7708 & 1.117 & 0.5018 & 2.226 \\
13.285 & 14.335 & 13.7785 & 1.050 & 0.4935 & 2.128 \\
13.331 & 14.414 & 13.841 & 1.083 & 0.510 & 2.124 \\
13.21 & 14.345 & 13.7441 & 1.135 & 0.5341 & 2.125 \\
13.316 & 14.213 & 13.7376 & 0.897 & 0.4216 & 2.128 \\
& & & & Average & 2.161 \\
\hline
\end{tabular}

${ }^{a}$ First three samples are SRNL-supplied resin. Average of SRNL resin F-factors is 2.197. Average of ORNL resin F-factors is 2.125. All resins were in hydrogen form.

Table 15. F-factor data for resin used in third equilibrations at $45^{\circ} \mathrm{C}$

\begin{tabular}{cccccc}
\hline $\begin{array}{c}\text { Empty vial } \\
\text { mass, } \mathbf{g}\end{array}$ & $\begin{array}{c}\text { Gross mass } \\
\text { w/damp resin, } \mathbf{g}\end{array}$ & $\begin{array}{c}\text { Gross mass } \\
\text { w/dry resin, g }\end{array}$ & $\begin{array}{c}\text { Damp resin } \\
\text { mass, g }\end{array}$ & $\begin{array}{c}\text { Dry resin } \\
\text { mass, g }\end{array}$ & F-factor $^{\boldsymbol{a}}$ \\
\hline 13.224 & 14.493 & 13.7728 & 1.269 & 0.5488 & 2.312 \\
13.286 & 14.495 & 13.8145 & 1.209 & 0.5285 & 2.288 \\
13.344 & 14.254 & 13.7478 & 0.910 & 0.4038 & 2.254 \\
13.314 & 14.343 & 13.7961 & 1.029 & 0.4821 & 2.134 \\
13.229 & 14.31 & 13.7374 & 1.081 & 0.5084 & 2.126 \\
13.387 & 14.411 & 13.8699 & 1.024 & 0.4829 & 2.121 \\
& & & & Average & 2.206 \\
\hline
\end{tabular}

${ }^{a} 1^{\text {st }}$ three samples are SRNL-supplied resin. Average of SRNL resin F-factors is 2.285. Average of ORNL resin F-factors is 2.127. All values are for resin in hydrogen form.

\subsubsection{Fourth Equilibrations-Contacts with AP-101 Simulant}

Having demonstrated considerable reproducibility in the experimental method, testing using a more realistic simulant commenced. The objective of this fourth set of equilibrations was to obtain data for verification and enhancement of the distribution isotherm using a simulant of waste stored in Tank AP-101 at the Hanford Site. Resin 1 pretreatment, sample collection, F-factor determination, and equilibrations were performed in the manners used in the previous two tests. Equilibrations were performed at $25^{\circ} \mathrm{C}$ and $45^{\circ} \mathrm{C}$ for periods of $72 \mathrm{~h}$. Temperature fluctuations during equilibration were similar to those observed in the preceding test. The nominal composition of the background simulant 
matrix is presented in Table 16. To evaluate the effect of ion exchange site loading on cesium uptake, equilibrations were performed at eight cesium concentrations ranging from $0.03 \mu M$ to $50 \mathrm{mM}$ (Table 17).

Of particular interest were partition coefficients obtained at low initial liquid-phase cesium concentrations. As in previous tests, duplicate equilibrations were performed at each combination of test conditions. The total number of samples collected was 36: 2 temperatures $\times 8$ Cs concentrations $\times 2$ samples per condition (duplicates) +4 random control duplicates to confirm consistency between analytical methods. In addition, two flasks without resin were "equilibrated" at $25^{\circ} \mathrm{C}$ to evaluate cesium adsorption by the polymer (polypropylene) containers. All feed solutions in testing were spiked with the same amount of activity; i.e., the differences in cesium concentration were the result of differences in the levels of stable cesium used.

The gamma-counting results from the blank samples indicate some degree of cesium adsorption by the test vessels. The initial feed solutions used in testing counted 25,350 counts $/ \mathrm{mL} / \mathrm{min}$ compared with

Table 16. AP-101 background simulant composition (nominal)

\begin{tabular}{lcc}
\hline $\begin{array}{c}\text { Simulant } \\
\text { component }\end{array}$ & Concentration, $\boldsymbol{M}$ & Concentration, ${ }^{a} \mathbf{g} / \mathbf{L}$ \\
\hline \multicolumn{3}{c}{ Compounds } \\
$\mathrm{NaNO}_{3}$ & 0.98 & \\
$\mathrm{NaNO}_{2}$ & 0.70 & 83.3 \\
$\mathrm{NaOH}$ & 1.94 & 48.3 \\
$\mathrm{NaAlO}$ & 0.30 & 77.6 \\
$\mathrm{Na}_{2} \mathrm{CO}_{3}$ & 0.40 & 24.6 \\
$\mathrm{Na}_{2} \mathrm{SO}_{4}$ & 0.04 & 42.4 \\
$\mathrm{NaCl}_{\mathrm{KNO}}$ & 0.04 & 5.7 \\
& 0.70 & 2.3 \\
& & 70.8 \\
$\mathrm{Na}$ & 4.84 & \\
$\mathrm{NO}_{3}$ & 1.68 & 111.3 \\
$\mathrm{NO}_{2}$ & 0.70 & 104.2 \\
$\mathrm{OH}$ & 1.94 & 32.2 \\
$\mathrm{Al}$ & 0.30 & 33.0 \\
$\mathrm{CO}$ & 0.4 & 8.1 \\
$\mathrm{SO}_{4}$ & 0.04 & 24.0 \\
$\mathrm{Cl}$ & 0.04 & 3.8 \\
$\mathrm{~K}$ & 0.70 & 1.4 \\
\hline${ }^{a}$ Concentrations are calculated based on masses of reagents added.
\end{tabular}

Table 17. Initial simulant cesium concentration (nominal)

\begin{tabular}{cc}
\hline Cesium conc., $\boldsymbol{M}$ & Cesium conc., $\mathbf{~ m g} / \mathbf{L}$ \\
\hline $5.0 \mathrm{E}-02$ & 6650 \\
$9.5 \mathrm{E}-03$ & 1260 \\
$1.6 \mathrm{E}-03$ & 213 \\
$2.6 \mathrm{E}-04$ & 34.6 \\
$4.5 \mathrm{E}-05$ & 5.98 \\
$5.0 \mathrm{E}-06$ & 0.665 \\
$8.1 \mathrm{E}-07$ & 0.108 \\
$3.0 \mathrm{E}-08$ & $3.99 \mathrm{E}-03$ \\
\hline
\end{tabular}


an average of 23,606 counts $/ \mathrm{mL} / \mathrm{min}$ for the two blanks after $72 \mathrm{~h}$ equilibration at $25^{\circ} \mathrm{C}$. The effects of a consistent shift of this magnitude on $\mathrm{D}_{\mathrm{Cs}}$ values are shown in the corrected and uncorrected values tabulated in Table 18. Any cesium sorption by the test vessels containing resin should be lower than that

Table 18. Results of fourth equilibrations-AP-101 simulant at $25^{\circ} \mathrm{C}$ and $45^{\circ} \mathrm{C}$

\begin{tabular}{|c|c|c|c|}
\hline $\begin{array}{c}\text { Simulant Cs } \\
\text { conc., } \\
M\end{array}$ & $\begin{array}{l}\text { Equilibration } \\
\text { temperature } \\
\text { (C) }\end{array}$ & $\begin{array}{l}\text { Cs distribution w/o } \\
\text { flask adsorption } \\
\text { correction }\end{array}$ & $\begin{array}{c}\text { Cs distribution } \\
\text { w/flask adsorption } \\
\text { correction }\end{array}$ \\
\hline $5.00 \mathrm{E}-02$ & 25 & 55.0 & 44.8 \\
\hline $5.00 \mathrm{E}-02$ & 25 & 59.0 & 48.5 \\
\hline $5.00 \mathrm{E}-02$ & 45 & 47.8 & NA \\
\hline $5.00 \mathrm{E}-02$ & 45 & 44.6 & NA \\
\hline $9.50 \mathrm{E}-03$ & 25 & 150.6 & 133.5 \\
\hline $9.50 \mathrm{E}-03$ & 25 & 155.45 & 138.1 \\
\hline $9.50 \mathrm{E}-03$ & 45 & 118.0 & NA \\
\hline $9.50 \mathrm{E}-03$ & 45 & 122.8 & NA \\
\hline $1.60 \mathrm{E}-03$ & 25 & 338.6 & 308.9 \\
\hline $1.60 \mathrm{E}-03$ & 25 & 332.7 & 303.6 \\
\hline $1.60 \mathrm{E}-03$ & 25 & 335.3 & 305.6 \\
\hline $1.60 \mathrm{E}-03$ & 45 & 242.2 & NA \\
\hline $1.60 \mathrm{E}-03$ & 45 & 208.5 & NA \\
\hline $1.60 \mathrm{E}-03$ & 45 & 242.9 & NA \\
\hline $2.60 \mathrm{E}-04$ & 25 & 558.2 & 513.6 \\
\hline $2.60 \mathrm{E}-04$ & 25 & 537.5 & 494.2 \\
\hline $2.60 \mathrm{E}-04$ & 45 & 330.4 & NA \\
\hline $2.60 \mathrm{E}-04$ & 45 & 323.1 & NA \\
\hline $4.50 \mathrm{E}-05$ & 25 & 647.7 & 597.1 \\
\hline $4.50 \mathrm{E}-05$ & 25 & 678.4 & 625.5 \\
\hline $4.50 \mathrm{E}-05$ & 25 & 619.6 & 571.1 \\
\hline $4.50 \mathrm{E}-05$ & 45 & 385.4 & NA \\
\hline $4.50 \mathrm{E}-05$ & 45 & 409.5 & NA \\
\hline $4.50 \mathrm{E}-05$ & 45 & 409.0 & NA \\
\hline $5.00 \mathrm{E}-06$ & 25 & 762.0 & 703.1 \\
\hline $5.00 \mathrm{E}-06$ & 25 & 743.4 & 686.4 \\
\hline $5.00 \mathrm{E}-06$ & 45 & 446.8 & NA \\
\hline $5.00 \mathrm{E}-06$ & 45 & 413.5 & NA \\
\hline $8.10 \mathrm{E}-07$ & 25 & 815.4 & 753.3 \\
\hline $8.10 \mathrm{E}-07$ & 25 & 759.0 & 700.7 \\
\hline $8.10 \mathrm{E}-07$ & 45 & 456.0 & NA \\
\hline $8.10 \mathrm{E}-07$ & 45 & 432.1 & NA \\
\hline $3.00 \mathrm{E}-08$ & 25 & 835.1 & 772.0 \\
\hline $3.00 \mathrm{E}-08$ & 25 & 790.4 & 730.3 \\
\hline $3.00 \mathrm{E}-08$ & 45 & 422.3 & NA \\
\hline $3.00 \mathrm{E}-08$ & 45 & 437.9 & NA \\
\hline
\end{tabular}


for the blank vessels, due to cesium sorption by the resin; therefore the corrected values represent a worstcase change.

As in all previous tests, results from duplicate tests are in close agreement. Consistent reductions in $\mathrm{D}_{\mathrm{Cs}}$ are observed with increasing initial cesium concentration. In addition, $\mathrm{D}_{\mathrm{Cs}}$ depression with increasing temperature is indicated, as was the case in the previous test. The effect of temperature is considerably more pronounced at lower cesium concentrations. Cesium uptakes at the $4.5 \times 10^{-5} \mathrm{M}$ concentration from the AP-101 background at both temperature conditions are significantly less than those from the simple background matrix used in the third equilibrations (Table 12), which contained a similar initial concentration of cesium. This disparity is expected, based on the higher concentration of potassium in the AP-101 simulant. The data obtained are presented in Fig. 10 and Fig. 11, in which they are compared with SRNL data and a predicted isotherm for uptake from AP-101 simulant. As shown, the data sets and predicted results are in relatively close agreement. There is, however, clear deviation in both SRNL and

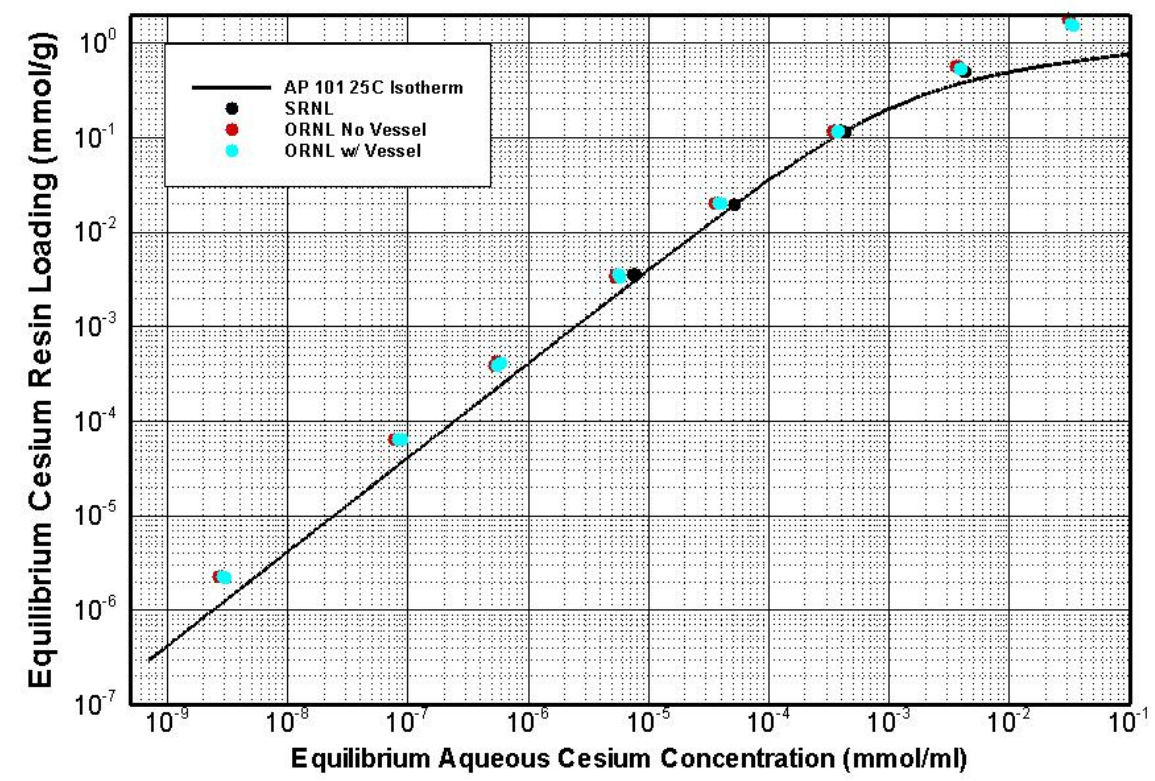

Fig. 10. Cesium loading results from AP-101 simulant at $25^{\circ} \mathrm{C}$.

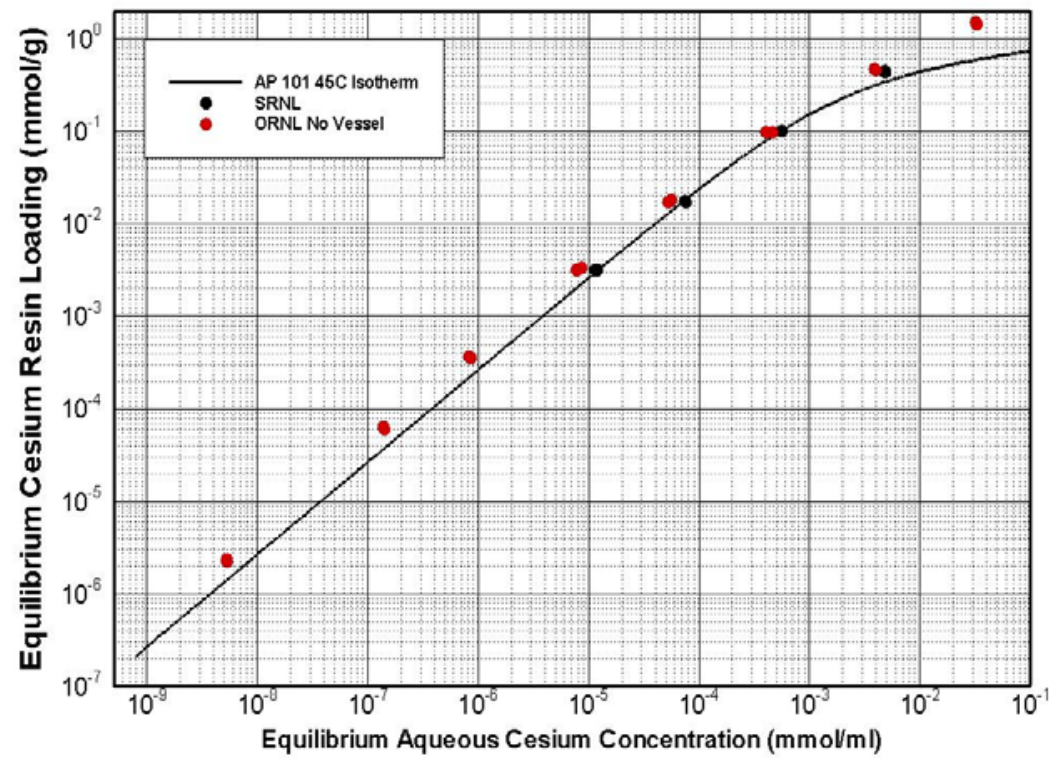

Fig. 11. Cesium loading results from AP-101 simulant at $45^{\circ} \mathrm{C}$. 
ORNL data from model predictions at low and high cesium concentrations, indicating that model refinements are necessary.

F-factors determined in conjunction with AP-101 equilibrations are presented in Table 19 and Table 20.

Table 19. F-factor data for resin used in 4 th equilibrations at $25^{\circ} \mathrm{C}$

\begin{tabular}{cccccc}
\hline $\begin{array}{c}\text { Empty vial } \\
\text { mass, } \\
\mathbf{g}\end{array}$ & $\begin{array}{c}\text { Gross mass } \\
\text { w/damp resin, } \\
\mathbf{g}\end{array}$ & $\begin{array}{c}\text { Gross mass } \\
\mathbf{w} / \text { dry resin, } \\
\mathbf{g}\end{array}$ & $\begin{array}{c}\text { Damp resin } \\
\text { mass, } \mathbf{g}\end{array}$ & $\begin{array}{c}\text { Dry resin } \\
\text { mass, g }\end{array}$ & F-factor \\
\hline 13.2378 & 14.265 & 13.6979 & 1.0272 & 0.4601 & 2.234 \\
13.2845 & 14.316 & 13.7612 & 1.0315 & 0.4767 & 2.164 \\
13.3286 & 14.578 & 13.9359 & 1.2494 & 0.6073 & 2.057 \\
& & & & Average & 2.151 \\
\hline
\end{tabular}

Table 20. F-factor data for resin used in 4 th equilibrations at $45^{\circ} \mathrm{C}$

\begin{tabular}{cccccc}
\hline $\begin{array}{c}\text { Empty vial } \\
\text { mass, } \\
\mathbf{g}\end{array}$ & $\begin{array}{c}\text { Gross mass } \\
\text { w/damp resin, } \\
\mathbf{g}\end{array}$ & $\begin{array}{c}\text { Gross mass } \\
\text { w/ dry resin, } \\
\mathbf{g}\end{array}$ & $\begin{array}{c}\text { Damp resin } \\
\text { mass, g }\end{array}$ & $\begin{array}{c}\text { Dry resin } \\
\text { mass, } \mathbf{g}\end{array}$ & F-factor \\
\hline 13.251 & 14.284 & 13.746 & 1.033 & 0.4950 & 2.087 \\
13.342 & 14.475 & 13.8927 & 1.133 & 0.5507 & 2.057 \\
13.301 & 14.474 & 13.8773 & 1.173 & 0.5763 & 2.035 \\
13.327 & 14.486 & 13.8636 & 1.159 & 0.5366 & 2.160 \\
& & & & Average & 2.085 \\
\hline
\end{tabular}

\subsubsection{Fifth Equilibrations-Extended Contact Test}

The objective of this test was to perform equilibrations at controlled (and elevated) temperatures over extended periods of time (approximately 3 weeks) to examine resin degradation effects, if any, on ion exchange performance. Extended contact testing and baseline stability evaluations were performed using simulant formulations that were modified to include free $\mathrm{OH}^{-}$at a concentration of $5 \mathrm{M}$ in order to accelerate degradation. The background simulant used was the AP-101 matrix described in Table 16, with the exception that the total $\mathrm{Na}$ ion concentration was $7.9 \mathrm{M}$ due to the intentional increase in hydroxide concentration that was accomplished by increasing the quantity of $\mathrm{NaOH}$ used in simulant formulation. Simulants containing cesium at two concentrations, $9.5 \times 10^{-3} \mathrm{M}$ and $4.5 \times 10^{-5} \mathrm{M}$, were used in this phase of testing. A preliminary test was performed prior to the equilibrations to confirm that increasing the $\mathrm{OH}^{-}$ concentration of the baseline AP-101 simulant to $5 \mathrm{M}$ by the addition of sodium hydroxide did not result in the formation of precipitates in the simulant solution.

Resin preparation and collection, and F-factor determinations were performed using the same methods as were applied in the previous three tests. In general, equilibrations were also performed in the manner used in these tests. However, resin/simulant contacts were conducted at temperature conditions different from those maintained in previous tests. Twenty-two resin samples were contacted with highhydroxide simulant (AP-101 simulant with the $\mathrm{NaOH}$ concentration increased to $5 \mathrm{M}$ ) for 3 weeks. Subsets of the resin samples were contacted at $25^{\circ} \mathrm{C}, 45^{\circ} \mathrm{C}$, and $65^{\circ} \mathrm{C}$. These subsets were further divided into contacts at the "high" and "low" cesium concentrations stated above. Two additional samples were not contacted with simulant for an extended period but were equilibrated for $72 \mathrm{~h}$ in the lower-cesium simulant at $25^{\circ} \mathrm{C}$ to establish a baseline for comparison because partitioning data from high $\mathrm{OH}^{-}$solutions at ambient temperature does not exist. Ten extended-contact samples (five test conditions, with duplicates) were allowed to re-equilibrate at $25^{\circ} \mathrm{C}$ for $72 \mathrm{~h}$ after the extended contact time, after which 
samples were collected for analysis. The remaining 12 extended-contact samples ( 6 conditions, with duplicates) were allowed to equilibrate for $72 \mathrm{~h}$ at $45^{\circ} \mathrm{C}$, after which samples were removed. Conditions for extended contact testing are summarized in Table 21. Results of F-factor determinations are given in Table 22 .

Table 21. Conditions for extended resin contact testing

\begin{tabular}{ccc}
\hline $\begin{array}{c}\text { Extended contact } \\
\text { temp. }\left({ }^{\circ} \mathbf{C}\right)\end{array}$ & $\begin{array}{r}\text { Final equilibration } \\
\text { temp. }\left({ }^{\circ} \mathbf{C}\right)\end{array}$ & $\begin{array}{c}\text { Simulant Cs conc., } \\
\boldsymbol{M}\end{array}$ \\
\hline 25 & 25 & $4.5 \times 10^{-5}$ \\
25 & 25 & $9.5 \times 10^{-3}$ \\
45 & 25 & $4.5 \times 10^{-5}$ \\
65 & 25 & $4.5 \times 10^{-5}$ \\
65 & 25 & $9.5 \times 10^{-3}$ \\
25 & 45 & $4.5 \times 10^{-5}$ \\
25 & 45 & $9.5 \times 10^{-3}$ \\
45 & 45 & $4.5 \times 10^{-5}$ \\
45 & 45 & $9.5 \times 10^{-3}$ \\
65 & 45 & $4.5 \times 10^{-5}$ \\
65 & 45 & $9.5 \times 10^{-3}$ \\
\hline
\end{tabular}

Table 22. F-factor data for resin used in extended contact equilibrations

\begin{tabular}{cccccc}
\hline $\begin{array}{c}\text { Empty vial } \\
\text { mass, } \mathbf{g}\end{array}$ & $\begin{array}{c}\text { Gross mass } \\
\text { w/damp resin, } \\
\mathbf{g}\end{array}$ & $\begin{array}{c}\text { Gross mass } \\
\mathbf{w} / \text { dry resin, } \\
\mathbf{g}\end{array}$ & $\begin{array}{c}\text { Damp resin } \\
\text { mass, }\end{array}$ & $\begin{array}{c}\text { Dry resin } \\
\text { mass, } \mathbf{g}\end{array}$ & F-factor $^{\boldsymbol{a}}$ \\
\hline 13.314 & 14.904 & 14.076 & 1.59 & 0.762 & 2.087 \\
13.34 & 14.581 & 13.972 & 1.241 & 0.632 & 1.964 \\
13.397 & 14.946 & 14.157 & 1.549 & 0.760 & 2.038 \\
13.335 & 14.505 & 13.8754 & 1.17 & 0.5404 & 2.165 \\
13.238 & 14.404 & 13.758 & 1.166 & 0.520 & 2.242 \\
13.265 & 14.375 & 13.7596 & 1.11 & 0.4946 & 2.244 \\
\hline
\end{tabular}

${ }^{a}$ Significant discrepancy existed from first three to second three samples due to the amount of time required to weigh all of the resin samples. The average of the first three and second three samples were applied to the appropriate equilibration samples. Average of first three F-factors is 2.029; average of second three F-factors is 2.217 .

The test results (Table 23) exhibit the expected changes in cesium uptake in response to changes in equilibration temperature and initial cesium concentration. Increases in both result in decreases in $\mathrm{D}_{\mathrm{Cs}}$. In addition, significant changes in cesium uptake were generated by allowing samples to re-equilibrate with a temperature change after the extended contact periods. 
Table 23. Extended contact test results (with AP-101 simulant)

\begin{tabular}{|c|c|c|c|c|c|}
\hline $\begin{array}{c}\text { 3-week contact } \\
\text { temperature, } \\
{ }^{\circ} \mathrm{C} \\
\end{array}$ & $\begin{array}{l}3 \text { day post-contact } \\
\text { equilibration } \\
\text { temperature, }{ }^{\circ} \mathrm{C}\end{array}$ & $\begin{array}{c}\text { Initial Cs } \\
\text { conc., } M\end{array}$ & $\begin{array}{c}\text { Raffinate Cs } \\
\text { conc., } \\
\text { mmol/mL }\end{array}$ & $\begin{array}{c}\text { Calculated } \\
\text { resin Cs conc., } \\
\text { mmol/g resin }\end{array}$ & $\begin{array}{c}\text { Cs distribution, } \\
\mathbf{D}_{\mathrm{Cs}}\end{array}$ \\
\hline 25 & 25 & $4.50 \mathrm{E}-05$ & $6.21 \mathrm{E}-06$ & $3.47 \mathrm{E}-03$ & 559.7 \\
\hline 25 & 25 & $4.50 \mathrm{E}-05$ & $5.96 \mathrm{E}-06$ & $3.32 \mathrm{E}-03$ & 556.6 \\
\hline 25 & 45 & $4.50 \mathrm{E}-05$ & $9.75 \mathrm{E}-06$ & $3.03 \mathrm{E}-03$ & 311.0 \\
\hline 25 & 45 & $4.50 \mathrm{E}-05$ & $9.29 \mathrm{E}-06$ & $3.06 \mathrm{E}-03$ & 329.5 \\
\hline 45 & 25 & $4.50 \mathrm{E}-05$ & $7.31 \mathrm{E}-06$ & $2.31 \mathrm{E}-03$ & 315.2 \\
\hline 45 & 25 & $4.50 \mathrm{E}-05$ & $6.88 \mathrm{E}-06$ & $3.20 \mathrm{E}-03$ & 465.8 \\
\hline 45 & 45 & $4.50 \mathrm{E}-05$ & $1.02 \mathrm{E}-05$ & $3.07 \mathrm{E}-03$ & 301.2 \\
\hline 45 & 45 & $4.50 \mathrm{E}-05$ & $1.02 \mathrm{E}-05$ & $3.00 \mathrm{E}-03$ & 292.5 \\
\hline 65 & 25 & $4.50 \mathrm{E}-05$ & $8.46 \mathrm{E}-06$ & $3.37 \mathrm{E}-03$ & 397.9 \\
\hline 65 & 25 & $4.50 \mathrm{E}-05$ & $8.91 \mathrm{E}-06$ & $3.37 \mathrm{E}-03$ & 378.5 \\
\hline 65 & 45 & $4.50 \mathrm{E}-05$ & $1.32 \mathrm{E}-05$ & $3.02 \mathrm{E}-03$ & 228.0 \\
\hline 65 & 45 & $4.50 \mathrm{E}-05$ & $1.35 \mathrm{E}-05$ & $3.03 \mathrm{E}-03$ & 224.6 \\
\hline 25 & 25 & $9.50 \mathrm{E}-03$ & 0.003672 & $5.04 \mathrm{E}-01$ & 137.3 \\
\hline 25 & 25 & $9.50 \mathrm{E}-03$ & 0.003834 & $5.15 \mathrm{E}-01$ & 134.4 \\
\hline 25 & 45 & $9.50 \mathrm{E}-03$ & 0.004354 & $4.72 \mathrm{E}-01$ & 108.3 \\
\hline 25 & 45 & $9.50 \mathrm{E}-03$ & 0.004121 & $4.63 \mathrm{E}-01$ & 112.2 \\
\hline 45 & 45 & $9.50 \mathrm{E}-03$ & 0.004451 & $4.29 \mathrm{E}-01$ & 96.3 \\
\hline 45 & 45 & $9.50 \mathrm{E}-03$ & 0.004193 & $4.47 \mathrm{E}-01$ & 106.6 \\
\hline 65 & 25 & $9.50 \mathrm{E}-03$ & 0.004396 & $4.87 \mathrm{E}-01$ & 110.9 \\
\hline 65 & 25 & $9.50 \mathrm{E}-03$ & 0.00445 & $4.66 \mathrm{E}-01$ & 104.7 \\
\hline 65 & 45 & $9.50 \mathrm{E}-03$ & 0.005185 & $4.07 \mathrm{E}-01$ & 78.5 \\
\hline 65 & 45 & $9.50 \mathrm{E}-03$ & 0.004954 & 4.23E-01 & 85.5 \\
\hline$a$ & 25 & $4.50 \mathrm{E}-05$ & $6.37 \mathrm{E}-06$ & $3.68 \mathrm{E}-03$ & 577.4 \\
\hline$a$ & 25 & $4.50 \mathrm{E}-05$ & $6.61 \mathrm{E}-06$ & $3.59 \mathrm{E}-03$ & 542.4 \\
\hline
\end{tabular}

${ }^{a}$ Results of control tests in which contact was limited to 3 days at $25^{\circ} \mathrm{C}$.

Comparable data from 3-day testing (the fourth equilibration) and extended testing are presented in Table 24. The data from the extended contact test presented in this table is from equilibrations in which the extended contact and re-equilibration temperatures were unchanged. The results presented in this table indicate the difficulty in direct comparison of extended contact test results with equilibrations of shorter duration conducted in the reported work. This difficulty arises from the higher level of sodium present in the simulant used for extended contact tests. As the data in Table 24 indicate, cesium removal from the solution containing $\mathrm{Na}^{+}$at the lower concentration is approximately $14 \%$ greater (in terms of $\mathrm{D}_{\mathrm{Cs}}$ ) than removal from the simulant used in the extended contact test, when all other conditions (contact time and temperature) are equal (i.e., 3-day contacts at $25^{\circ} \mathrm{C}$ ). The difference is indicative of sodium competition with cesium for bonding sites. 
Table 24. Comparison of extended and standard contact results

\begin{tabular}{ccccc}
\hline $\begin{array}{c}\text { Equilibration } \\
\text { temperature, }{ }^{\circ} \mathbf{C}\end{array}$ & Contact time & $\begin{array}{c}\text { Initial Cs } \\
\text { conc., } \boldsymbol{M}\end{array}$ & $\begin{array}{c}\mathbf{O H}^{-} \text {conc., } \\
\boldsymbol{M}\end{array}$ & $\begin{array}{c}\text { Cs distribution, } \\
\mathbf{D}_{\mathrm{Cs}}{ }^{a}\end{array}$ \\
\hline 25 & $3 \mathrm{wk}+3 \mathrm{~d}$ & $4.50 \mathrm{E}-05$ & 5 & 560 \\
25 & $3 \mathrm{wk}+3 \mathrm{~d}$ & $4.50 \mathrm{E}-05$ & 5 & 557 \\
25 & $3 \mathrm{~d}$ & $4.50 \mathrm{E}-05$ & 1.94 & $648^{b}$ \\
25 & $3 \mathrm{~d}$ & $4.50 \mathrm{E}-05$ & 1.94 & $678^{b}$ \\
25 & $3 \mathrm{~d}$ & $4.50 \mathrm{E}-05$ & 1.94 & $620^{b}$ \\
25 & $3 \mathrm{~d}$ & $4.50 \mathrm{E}-05$ & 5 & 577 \\
25 & $3 \mathrm{~d}$ & $4.50 \mathrm{E}-05$ & 5 & 542 \\
25 & $3 \mathrm{wk}+3 \mathrm{~d}$ & $9.50 \mathrm{E}-03$ & 5 & 137 \\
25 & $3 \mathrm{wk}+3 \mathrm{~d}$ & $9.50 \mathrm{E}-03$ & 5 & 134 \\
45 & $3 \mathrm{wk}+3 \mathrm{~d}$ & $4.50 \mathrm{E}-05$ & 5 & 301 \\
45 & $3 \mathrm{wk}+3 \mathrm{~d}$ & $4.50 \mathrm{E}-05$ & 5 & 293 \\
45 & $3 \mathrm{~d}$ & $4.50 \mathrm{E}-05$ & 1.94 & 385 \\
45 & $3 \mathrm{~d}$ & $4.50 \mathrm{E}-05$ & 1.94 & 410 \\
45 & $3 \mathrm{~d}$ & $4.50 \mathrm{E}-05$ & 1.94 & 409 \\
45 & $3 \mathrm{wk}+3 \mathrm{~d}$ & $9.50 \mathrm{E}-03$ & 5 & 96 \\
45 & $3 \mathrm{wk}+3 \mathrm{~d}$ & $9.50 \mathrm{E}-03$ & 5 & 107 \\
45 & $3 \mathrm{~d}$ & $9.50 \mathrm{E}-03$ & 1.94 & 118 \\
45 & $3 \mathrm{~d}$ & $9.50 \mathrm{E}-03$ & 1.94 & 123 \\
\hline
\end{tabular}

${ }^{a}$ All resins used were initially in hydrogen form.

${ }^{\mathrm{b}}$ Values from the fourth equilibration are not corrected for vessel adsorption.

In the one instance in which short and extended contact tests were performed with the same simulant containing the same initial cesium concentration $\left(25^{\circ} \mathrm{C}\right.$ contacts at $\left.4.5 \times 10^{-5} \mathrm{M} \mathrm{Cs}\right)$, the increased contact time had no significant effect on cesium uptake; $\mathrm{D}_{\mathrm{Cs}}$ values for short and extended contacts are within experimental error. Similarly, when results from 3-day contacts (Table 18) are compared with extended contact results (Table 23), similar effects of temperature and initial cesium concentration are apparent. Increasing the cesium concentration from $4.5 \times 10^{-5} \mathrm{M}$ to $9.5 \times 10^{-3} \mathrm{M}$ results in greater than $60 \%$ decreases in $\mathrm{D}_{\mathrm{Cs}}$. Increasing the contact temperature from $25^{\circ} \mathrm{C}$ to $45^{\circ} \mathrm{C}$ resulted in a $25-30 \%$ decrease in cesium uptake at $9.5 \times 10^{-3} \mathrm{M}$ initial $\mathrm{Cs}$ and a decrease of approximately $40 \%$ in cesium removal resulting at $4.5 \times 10^{-5} \mathrm{M}$ initial cesium concentration, regardless of contact duration. The general conclusion drawn from the data is that the extended contact did not significantly alter resin performance.

While the observed reduction is Cs uptake over the contact periods used in this test was insignificant, there was an observed change in the physical appearance of the resin/simulant slurries during testing. The simulants took on a distinct purple color, seen in Fig. 12. No analysis of the simulants for resin degradation products was performed to determine the component(s) responsible for the color change. A photograph (Fig. 13) of resin that was equilibrated with the SRS simulant (Table 11) for $72 \mathrm{~h}$ is included for comparison. 


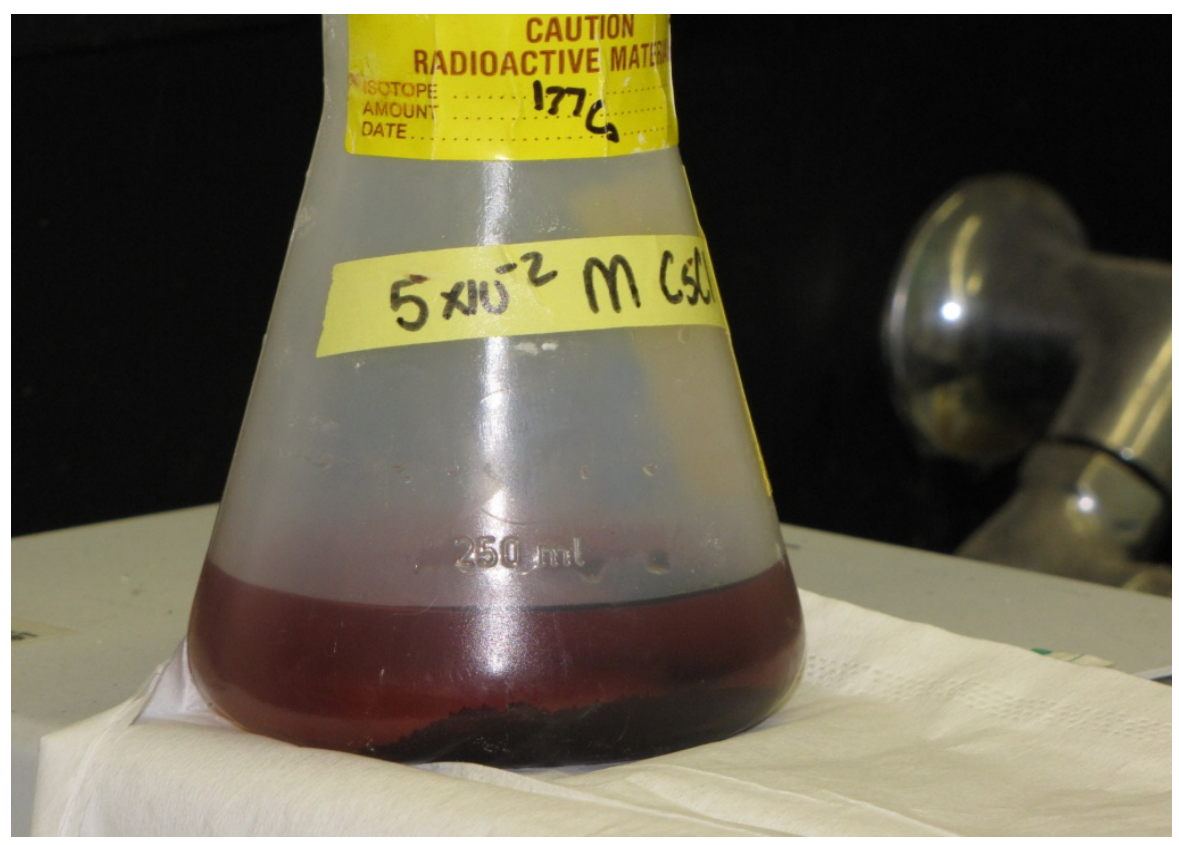

Fig. 12. Resin and simulant after 3 week contact with AP-101 simulant in $5 \mathrm{M} \mathrm{OH}^{-}$.

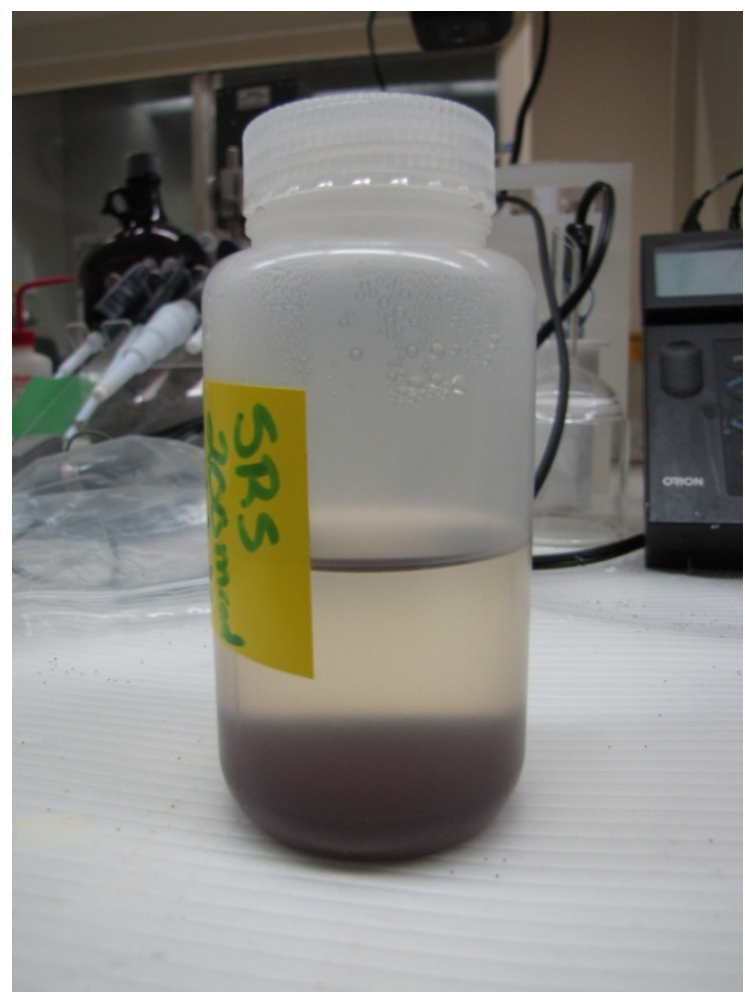

Fig. 13. Resin and simulant after 3 day contact with SRS simulant. 


\subsubsection{Radiolysis $K_{d}$ Tests}

For the cesium distribution measurements of the resin samples from the radiolysis tests, the same procedures were followed as for the standard distribution tests. The standard AP-101 simulant (1.94 M $\mathrm{NaOH}$ ) was used with a standard cesium concentration of $4.5 \mathrm{E}-5 \mathrm{M}$ and the ratio of dry resin to simulant was 1:100 at a temperature of $25^{\circ} \mathrm{C}$. All of the F-factors were performed in triplicate and the F-factors were averaged for each liquid/dose pair. The F-factors can be seen in Table 25 for the HFIR tests and in Table 26 for the Co-60 samples.

The cesium distributions were calculated in the same manner as for previous distribution tests. The simulant was spiked with radioactive cesium, and the gamma radiation was counted. The simulant was counted before and after contact with the resin. The amount of cesium deposited on the resin was calculated via subtraction. All distribution tests were performed in triplicate and the distributions were averaged for each liquid/dose pair.

Cesium distribution data from the HFIR and the Co-60 source experiments are compiled in Table 27 below. The 'liquid' column signifies the liquid the specific sample was irradiated in. After the irradiation, the resin was separated from the liquid and then converted to the hydrogen form. The resin was then dewatered and weighed for the distribution tests in AP-101 simulant.

One reason why the Co-60 source samples might have higher distribution coefficients than the HFIR samples is the temperature at which they were irradiated. The Co-60 source samples were at ambient temperature, while the HFIR samples were irradiated at temperatures ranging from $48^{\circ} \mathrm{C}$ to $51^{\circ} \mathrm{C}$. The higher temperature in the HFIR samples could have degraded the resin.

Resin clumping was only seen with the used resin, which had reacted with 7 mmoles oxygen/g dry resin prior to irradiation; all other resin samples were free flowing.

\subsection{TITRATIONS}

Three sets of titrations were conducted. An initial scoping test was performed to evaluate, qualitatively, the effect of counter-ion $\left(\mathrm{OH}^{-}, \mathrm{Cl}^{-}\right.$, and $\left.\mathrm{NO}_{3}^{-}\right)$on sodium uptake in order to determine the most effective sodium compound(s) with respect to resin loading. The results are also to be used to verify previously determined sodium capacities. The second test component comprised titrations using $\mathrm{NaOH}$ in a range of concentrations $(0.001 M$ to $6 M)$ to evaluate the effect of sodium availability on uptake. During this phase of testing the resin-to-titrant ratio was adjusted to mitigate against sodium cation saturation effects on sodium uptake. The third phase of testing was performed with titrant consisting of $\mathrm{NaOH}$ and $\mathrm{NaNO}_{3}$ at various concentration combinations yielding a total sodium concentration of $6 M$ (except in one case where $11 \mathrm{M} \mathrm{Na}$ was used). The objective of the testing was to determine, quantitatively, the effect of counter-ion concentration from a neutral salt on sodium uptake. Selection of $\mathrm{NaNO}_{3}$ for use in this phase of testing was made based on the lack of any significant effect when $\mathrm{NaCl}$ or $\mathrm{NaNO}_{3}$ was used (in the scoping test) and the predominance of nitrate ion in the waste being treated.

All titration tests were performed at ambient temperature (nominally $25^{\circ} \mathrm{C}$ ). 
Table 25. F-factors for HFIR samples

\begin{tabular}{|c|c|c|c|c|c|c|c|c|}
\hline Liquid & $\begin{array}{l}\text { Dose } \\
\text { (Mrad) }\end{array}$ & $\begin{array}{c}\text { Empty vial } \\
\text { tare (g) }\end{array}$ & $\begin{array}{c}\text { Vial with } \\
\text { damp RF (g) }\end{array}$ & $\begin{array}{l}\text { Vial with } \\
\text { dry RF (g) }\end{array}$ & $\underset{\text { (g) }}{\operatorname{damp} R F}$ & $\begin{array}{c}\text { dry RF } \\
\text { (g) }\end{array}$ & F-factor & $\begin{array}{l}\text { F-factor } \\
\text { average }\end{array}$ \\
\hline \multirow{3}{*}{$\mathrm{HNO}_{3}$} & 300 & 6.419 & 7.088 & 6.703 & 0.669 & 0.284 & 2.3556 & \\
\hline & 300 & 6.337 & 7.478 & 6.847 & 1.141 & 0.510 & 2.2373 & 2.22 \\
\hline & 300 & 6.339 & 7.530 & 6.913 & 1.191 & 0.574 & 2.0749 & \\
\hline \multirow{3}{*}{$\mathrm{HNO}_{3}$} & 250 & 6.457 & 7.621 & 6.972 & 1.164 & 0.515 & 2.2602 & \\
\hline & 250 & 6.340 & 7.501 & 6.855 & 1.161 & 0.515 & 2.2544 & 2.25 \\
\hline & 250 & 6.451 & 7.509 & 6.923 & 1.058 & 0.472 & 2.2415 & \\
\hline \multirow{3}{*}{$\mathrm{HNO}_{3}$} & 200 & 6.317 & 7.682 & 6.931 & 1.365 & 0.614 & 2.2231 & \\
\hline & 200 & 6.371 & 7.891 & 7.059 & 1.520 & 0.688 & 2.2093 & 2.21 \\
\hline & 200 & 6.374 & 7.626 & 6.948 & 1.252 & 0.574 & 2.1812 & \\
\hline \multirow{3}{*}{$\mathrm{HNO}_{3}$} & 150 & 6.352 & 7.985 & 7.100 & 1.633 & 0.748 & 2.1832 & \\
\hline & 150 & 6.339 & 7.829 & 7.048 & 1.490 & 0.709 & 2.1016 & 2.13 \\
\hline & 150 & 6.270 & 7.722 & 6.962 & 1.452 & 0.692 & 2.0983 & \\
\hline \multirow{3}{*}{ Water } & 300 & 6.290 & 7.522 & 6.822 & 1.232 & 0.532 & 2.3158 & \\
\hline & 300 & 6.283 & 7.598 & 6.849 & 1.315 & 0.566 & 2.3233 & 2.33 \\
\hline & 300 & 6.440 & 7.409 & 6.853 & 0.969 & 0.413 & 2.3462 & \\
\hline \multirow{3}{*}{ Water } & 250 & 6.387 & 7.556 & 6.900 & 1.169 & 0.513 & 2.2788 & \\
\hline & 250 & 6.441 & 7.611 & 6.959 & 1.170 & 0.518 & 2.2587 & 2.27 \\
\hline & 250 & 6.382 & 7.358 & 6.814 & 0.976 & 0.432 & 2.2593 & \\
\hline \multirow{3}{*}{ Water } & 200 & 6.428 & 7.545 & 6.872 & 1.117 & 0.444 & 2.5158 & \\
\hline & 200 & 6.422 & 7.510 & 6.888 & 1.088 & 0.466 & 2.3348 & 2.43 \\
\hline & 200 & 6.333 & 7.412 & & & & & \\
\hline \multirow{4}{*}{ Water } & 150 & 6.409 & 7.378 & 6.832 & 0.969 & 0.423 & 2.2908 & \\
\hline & 150 & 6.456 & 7.558 & 6.934 & 1.102 & 0.478 & 2.3054 & 2.29 \\
\hline & 150 & 6.304 & 7.218 & 6.704 & 0.914 & 0.400 & 2.2850 & \\
\hline & 100 & 6.365 & 7.168 & 6.730 & 0.803 & 0.365 & 2.2000 & \\
\hline
\end{tabular}


Table 25 (continued)

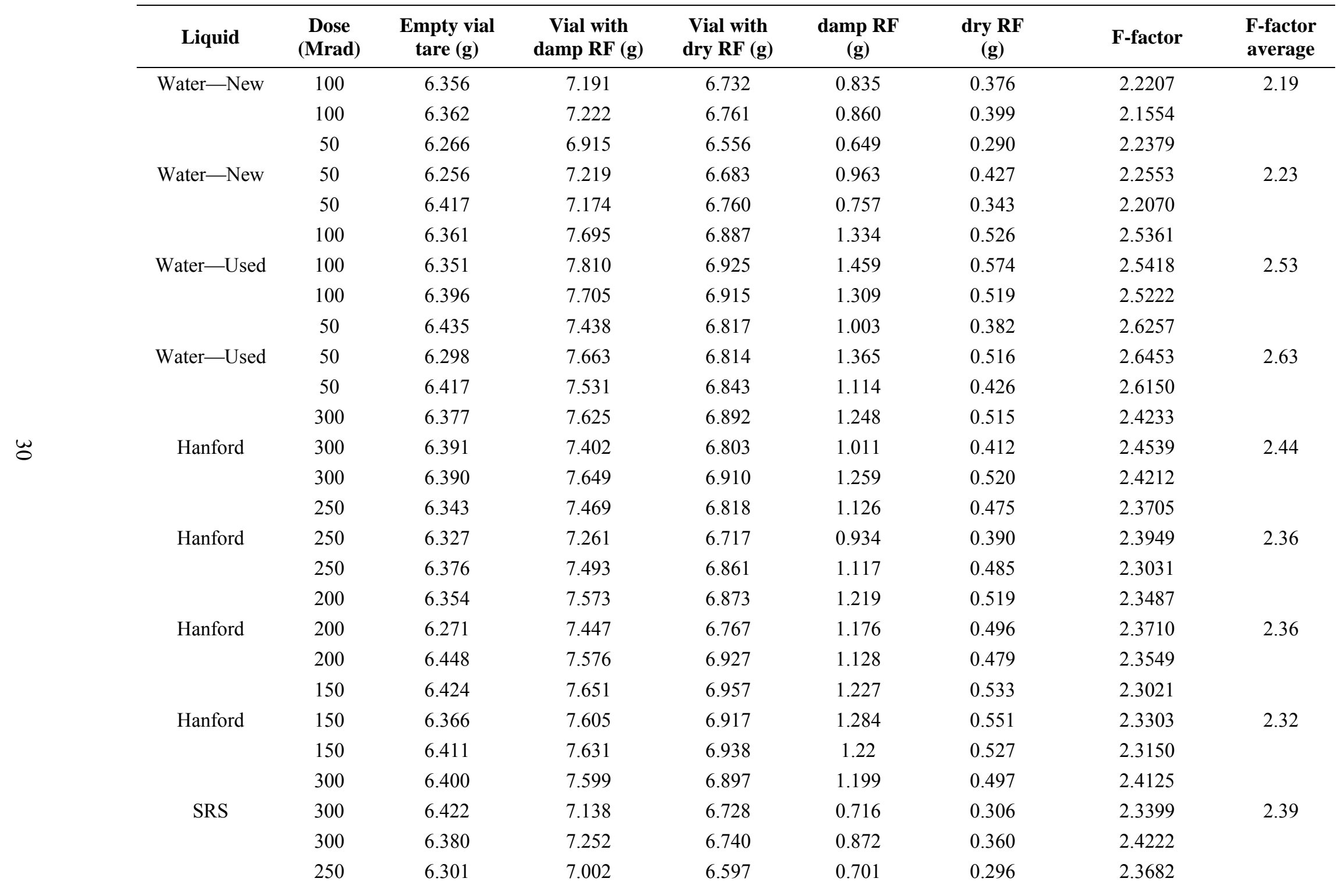


Table 25 (continued)

\begin{tabular}{|c|c|c|c|c|c|c|c|c|}
\hline Liquid & $\begin{array}{c}\text { Dose } \\
\text { (Mrad) }\end{array}$ & $\begin{array}{c}\text { Empty vial } \\
\text { tare (g) }\end{array}$ & $\begin{array}{c}\text { Vial with } \\
\text { damp RF (g) }\end{array}$ & $\begin{array}{c}\text { Vial with } \\
\text { dry RF (g) }\end{array}$ & $\begin{array}{c}\operatorname{damp} R F \\
\text { (g) }\end{array}$ & $\begin{array}{c}\text { dry RF } \\
\text { (g) }\end{array}$ & F-factor & $\begin{array}{l}\text { F-factor } \\
\text { average }\end{array}$ \\
\hline \multirow[t]{3}{*}{ SRS } & 250 & 6.434 & 7.392 & 6.850 & 0.958 & 0.416 & 2.3029 & 2.35 \\
\hline & 250 & 6.379 & 7.347 & 6.785 & 0.968 & 0.406 & 2.3842 & \\
\hline & 200 & 6.344 & 7.111 & 6.654 & 0.767 & 0.310 & 2.4742 & \\
\hline \multirow[t]{3}{*}{ SRS } & 200 & 6.394 & 7.219 & 6.724 & 0.825 & 0.303 & 2.5000 & 2.48 \\
\hline & 200 & 6.295 & 7.104 & 6.637 & 0.845 & 0.342 & 2.4708 & \\
\hline & 150 & 6.449 & 7.001 & 6.738 & 0.651 & 0.289 & 2.2526 & \\
\hline \multirow[t]{2}{*}{ SRS } & 150 & 6.378 & 7.170 & & & & & 2.26 \\
\hline & 150 & 6.418 & 7.042 & 6.693 & 0.624 & 0.275 & 2.2691 & \\
\hline
\end{tabular}


Table 26. F-factors for Co-60 source samples

\begin{tabular}{|c|c|c|c|c|c|c|c|c|}
\hline Liquid & $\begin{array}{l}\text { Dose } \\
\text { [Mrad] }\end{array}$ & $\begin{array}{c}\text { Empty vial } \\
\text { tare (g) }\end{array}$ & $\begin{array}{c}\text { Vial with } \\
\text { damp RF (g) }\end{array}$ & $\begin{array}{l}\text { Vial with } \\
\text { dry RF (g) }\end{array}$ & $\underset{\text { (g) }}{\operatorname{damp} R F}$ & $\begin{array}{c}\text { dry RF } \\
\text { (g) }\end{array}$ & F-factor & $\begin{array}{l}\text { F-factor } \\
\text { average }\end{array}$ \\
\hline \multirow{3}{*}{$\mathrm{HNO}_{3}$} & 300 & 6.414 & 7.123 & 6.771 & 0.709 & 0.357 & 1.9860 & \multirow{3}{*}{2.00} \\
\hline & 300 & 6.343 & 7.264 & 6.806 & 0.921 & 0.463 & 1.9892 & \\
\hline & 300 & 6.364 & 7.394 & 6.807 & 1.003 & 0.506 & 2.0356 & \\
\hline \multirow{3}{*}{ Water } & 300 & 6.401 & 7.274 & 6.812 & 0.873 & 0.411 & 2.1241 & \multirow{3}{*}{2.09} \\
\hline & 300 & 6.403 & 7.147 & 6.765 & 0.744 & 0.362 & 2.0552 & \\
\hline & 300 & 6.372 & 7.116 & 6.726 & 0.744 & 0.354 & 2.1017 & \\
\hline \multirow{3}{*}{ SRS } & 300 & 6.368 & 7.019 & 6.869 & 0.651 & 0.501 & 1.2994 & \multirow{3}{*}{1.89} \\
\hline & 300 & 6.439 & 7.215 & 6.792 & 0.776 & 0.353 & 2.1983 & \\
\hline & 300 & 6.445 & 7.144 & 6.766 & 0.699 & 0.321 & 2.1776 & \\
\hline \multirow{3}{*}{$\mathrm{HNO}_{3}$ control } & 0 & 6.394 & 7.041 & 6.727 & 0.647 & 0.333 & 1.9429 & \multirow{3}{*}{1.97} \\
\hline & 0 & 6.375 & 7.140 & 6.756 & 0.765 & 0.381 & 2.0079 & \\
\hline & 0 & 6.352 & 7.198 & 6.782 & 0.846 & 0.430 & 1.9674 & \\
\hline \multirow{3}{*}{ Water control } & 0 & 6.383 & 7.021 & 6.693 & 0.638 & 0.310 & 2.0581 & \multirow{3}{*}{2.06} \\
\hline & 0 & 6.335 & 7.077 & 6.696 & 0.742 & 0.361 & 2.0554 & \\
\hline & 0 & 6.312 & 6.96 & 6.626 & 0.648 & 0.314 & 2.0637 & \\
\hline \multirow{3}{*}{ SRS control } & 0 & 6.509 & 7.259 & 6.872 & 0.75 & 0.363 & 2.0661 & \multirow{3}{*}{2.07} \\
\hline & 0 & 6.429 & 7.076 & 6.742 & 0.647 & 0.313 & 2.0671 & \\
\hline & 0 & 6.370 & 7.124 & 6.734 & 0.754 & 0.364 & 2.0714 & \\
\hline
\end{tabular}


Table 27. Cesium distribution data from HFIR and Co-60 source

\begin{tabular}{|c|c|c|c|c|}
\hline Liquid & $\begin{array}{l}\text { Dose } \\
\text { (Mrad) }\end{array}$ & $\begin{array}{c}\text { HFIR } \\
\text { samples } \\
\mathbf{D}_{\mathrm{Cs}}\end{array}$ & $\begin{array}{c}\text { Co-60 source } \\
\text { samples } \\
D_{\mathrm{Cs}}\end{array}$ & $\begin{array}{c}\text { Co-60 source } \\
\text { controls } \\
D_{\mathrm{Cs}}\end{array}$ \\
\hline \multirow[t]{4}{*}{ Nitric Acid } & 300 & 3770 & 2575 & 6124 \\
\hline & 250 & 3003 & & \\
\hline & 200 & 3000 & & \\
\hline & 150 & 4930 & & \\
\hline \multirow[t]{8}{*}{ Water } & 300 & 3271 & 7780 & 9914 \\
\hline & 250 & 4773 & & \\
\hline & 200 & 1866 & & \\
\hline & 150 & 1755 & & \\
\hline & 100 (new) & 14489 & & \\
\hline & 50 (new) & 6580 & & \\
\hline & 100 (used) & 8172 & & \\
\hline & 50 (used) & 8105 & & \\
\hline \multirow[t]{4}{*}{ Hanford } & 300 & 4433 & N/A & N/A \\
\hline & 250 & 2976 & & \\
\hline & 200 & 4894 & & \\
\hline & 150 & 5186 & & \\
\hline \multirow[t]{4}{*}{ SRS } & 300 & 2764 & 3164 & 12423 \\
\hline & 250 & 3096 & & \\
\hline & 200 & 4331 & & \\
\hline & 150 & 4418 & & \\
\hline
\end{tabular}

\subsubsection{Initial (Scoping) Test}

Direct comparison of sodium uptake from $\mathrm{NaOH}, \mathrm{NaCl}$, and $\mathrm{NaNO}_{3}$ solutions containing sodium at a total concentration of $6 \mathrm{M}$ was performed. All solutions contained a minimum $\mathrm{NaOH}$ concentration of $1.0 M$ to ensure a $\mathrm{pH}$ of at least 14. Compositions of the three solutions used are listed in Table 28.

Solutions were prepared by dissolving ACS grade reagents in water that was deionized suing a Barnstead Nanopure II deionizer. The hydroxide concentration of test solutions was determined prior to use and after equilibration using a $\mathrm{pH}$ meter. Samples were diluted as needed to bring the $\mathrm{pH}$ into the $2-12$ range required for accurate analysis.

Table 28. Target compositions for scoping tests to evaluate anion effects

\begin{tabular}{ccc}
\hline $\begin{array}{c}\text { NaOH conc., } \\
\boldsymbol{M}\end{array}$ & $\begin{array}{c}\text { NaCl conc., } \\
\boldsymbol{M}\end{array}$ & $\begin{array}{c}\mathbf{N a N O}_{3} \text { conc., } \\
\boldsymbol{M}\end{array}$ \\
\hline 6.0 & 0.0 & 0.0 \\
1.0 & 5.0 & 0.0 \\
1.0 & 0.0 & 5.0 \\
\hline
\end{tabular}

During the scoping tests (Table 29), agitation was suspended after $1 \mathrm{~h}$ and the $\mathrm{pH}$ of the slurry was determined using a Thermo Electron Corp. ORION 720A $+\mathrm{pH}$ meter. Periods of agitation were repeated in one-hour increments until equilibrium was attained as indicated by identical $\mathrm{pH}$ measurements following two consecutive equilibration periods. Duplicate $\mathrm{pH}$ determinations were made for each testing at each titrant composition; one replicate test was performed at each test condition. Initial scoping test titrant compositions based on analytical results are presented in Table 30 . There is a significant disparity 
Table 29. Scoping test results

\begin{tabular}{|c|c|c|c|c|c|c|c|}
\hline Test no. & $\begin{array}{l}\text { Resin mass } \\
\text { (dry), g }\end{array}$ & $\begin{array}{c}\text { Titrant } \\
\text { volume, } \\
\text { mL }\end{array}$ & $\begin{array}{c}\text { Final }\left[\mathrm{OH}^{-}\right] \text {, } \\
M\end{array}$ & $\begin{array}{l}\text { Final titrant } \\
{\left[\mathrm{Na}^{+}\right], \mu \mathrm{g} / \mathrm{mL}}\end{array}$ & $\begin{array}{c}\mathrm{Na}^{+} \text {uptake based } \\
\text { on } \mathrm{Na}^{+} \text {analysis, } \\
\mathrm{mmol} / \mathrm{g} \text { resin }\end{array}$ & $\begin{array}{c}\mathrm{Na}^{+} \text {uptake based } \\
\text { on nominal } \mathrm{Na}^{+} \text {, } \\
\text { mmol/g resin }\end{array}$ & $\begin{array}{c}\mathrm{Na}^{+} \text {uptake based } \\
\text { on } \mathrm{OH}^{-} \text {depletion, } \\
\text { mmol/g resin }\end{array}$ \\
\hline 1 & 7.85 & 50 & 3.36 & 84500 & 5.12 & 14.81 & 10.86 \\
\hline 2 & 7.84 & 50 & 3.44 & 83100 & 5.52 & 15.21 & 10.37 \\
\hline 3 & 7.85 & 50 & 0.08 & 18400 & 5.01 & 33.12 & 5.49 \\
\hline 4 & 7.86 & 50 & 0.06 & 17100 & 5.37 & 33.44 & 5.59 \\
\hline 5 & 7.82 & 50 & 0.08 & 17800 & 5.40 & 33.41 & 5.55 \\
\hline 6 & 7.85 & 50 & 0.08 & 18100 & 5.29 & 33.20 & 5.57 \\
\hline
\end{tabular}

Table 30. Analytical results for titrants used in scoping test

\begin{tabular}{|c|c|c|c|c|}
\hline Test no. & $\begin{array}{c}\text { Nominal initial } \\
{\left[\mathrm{OH}^{-}\right], M}\end{array}$ & $\begin{array}{l}\text { Nominal initial } \\
\text { neutral salt, } M\end{array}$ & $\begin{array}{c}\text { Measured } \\
\text { initial }\left[\mathrm{OH}^{-}\right], M\end{array}$ & $\begin{array}{c}\text { Measured } \\
\text { initial }\left[\mathrm{Na}^{+}\right], M\end{array}$ \\
\hline 1 & 6.0 & 0.0 & 5.06 & 4.48 \\
\hline Duplicate & 6.0 & 0.0 & & \\
\hline 2 & 6.0 & 0.0 & & \\
\hline Duplicate & 6.0 & 0.0 & & \\
\hline 3 & 1.0 & $5.0 \mathrm{Cl}^{-}$ & 0.94 & 1.59 \\
\hline Duplicate & 1.0 & $5.0 \mathrm{Cl}^{-}$ & & \\
\hline 4 & 1.0 & $5.0 \mathrm{Cl}^{-}$ & & \\
\hline Duplicate & 1.0 & $5.0 \mathrm{Cl}^{-}$ & & \\
\hline 5 & 1.0 & $5.0 \mathrm{NO}_{3}^{-}$ & 0.95 & 1.62 \\
\hline Duplicate & 1.0 & $5.0 \mathrm{NO}_{3}^{-}$ & & \\
\hline 6 & 1.0 & $5.0 \mathrm{NO}_{3}^{-}$ & & \\
\hline Duplicate & 1.0 & $5.0 \mathrm{NO}_{3}^{-}$ & & \\
\hline
\end{tabular}


between target sodium concentrations and analytical results. While inconsistencies in sodium analysis results from other tests were observed, none were of the magnitude shown in Table 30. The source of the error is not known.

Results of scoping titrations are presented in Table 31. All resin loading results have been derived by subtraction of post-equilibration titrant concentrations from initial titrant conditions. The large

discrepancy between measured and nominal, initial sodium concentrations produces a similar discrepancy between results calculated by subtraction using the two different bases (analytical versus nominal feed values). However, comparison of results produced by titrations using $\mathrm{NaCl}$ or $\mathrm{NaNO}_{3}$ calculated from the same basis indicate that the sodium counterion has no effect on sodium transfer, i.e. differences are within experimental error.

Table 31. Nominal compositions for tests without salt background matrix

\begin{tabular}{ccc}
\hline $\begin{array}{c}\text { Initial NaOH } \\
\text { conc., } \boldsymbol{M}\end{array}$ & $\begin{array}{c}\text { Calculated } \\
\text { initial } \mathbf{~ p H}\end{array}$ & $\begin{array}{c}\text { Phase ratio } \\
\text { (mL liq:g dry solid) }\end{array}$ \\
\hline 6.0 & 14.8 & 4 \\
1.0 & 14.0 & 15 \\
0.1 & 13.0 & 100 \\
0.01 & 12.0 & 750 \\
0.001 & 11.0 & 5000 \\
\hline
\end{tabular}

\subsubsection{NaOH-only Titration Procedure}

Solutions used in testing were prepared as in the previous test; nominal concentrations are presented in Table 31. Target titrant-to-resin ratios were selected so as to avoid saturation effects, provide measurable differences in sodium concentration, and provide final hydroxide concentrations in the desired range.

As in Sect. 4.3.1, resin/titrant slurries were agitated for periods of $1 \mathrm{~h}$ using an enclosed orbital shaker controlled at $25^{\circ} \mathrm{C}$. (During testing, temperatures ranged from $25^{\circ} \mathrm{C}$ to $26^{\circ} \mathrm{C}$.) Slurry $\mathrm{pH}$ was measured after 1-h agitation periods, with agitation cycles repeated until equilibrium was reached.

Analytical results for titrants used in the $\mathrm{NaOH}$-only titrations are presented in Table 32. Titration results are shown in Table 33 and presented graphically in Fig. 14. As was the case in the scoping studies, sodium concentrations determined by ICP-atomic emission spectroscopy (AES) determinations at ORNL were not consistent with the target values (those based on mass of reagent used in solution preparation). Like the scoping study results, sodium concentrations in the titrants determined at ORNL were significantly lower than the target values in the case of higher $\mathrm{Na}$ levels, and are inconsistent with $\mathrm{Na}$ concentrations inferred from hydroxide determinations. At the higher initial $\mathrm{Na}$ concentration condition, ICP-AES results from the raffinate samples were also lower than the values inferred from the hydroxide analyses, causing the resin loading calculation results based on sodium and hydroxide concentrations to be similar.

Table 32. Analytical results for titrants used in NaOH-only titrations

\begin{tabular}{cccccc}
\hline $\begin{array}{c}\text { Nominal } \\
{\left[\mathbf{O H}^{-}\right], \boldsymbol{M}}\end{array}$ & $\begin{array}{c}\text { Avg. initial } \\
{\left[\mathbf{O H}^{-}\right], \boldsymbol{M}}\end{array}$ & $\begin{array}{c}\text { ORNL } \\
\text { measured initial } \\
{\left[\mathbf{N a}^{+}\right], \boldsymbol{\mu g} / \mathbf{m L}}\end{array}$ & $\begin{array}{c}\text { ORNL } \\
\text { measured } \\
\text { initial }\left[\mathbf{N a}^{+}\right], \boldsymbol{M}\end{array}$ & $\begin{array}{c}\text { SRNL measured } \\
\text { initial }\left[\mathbf{N a}^{+}\right], \\
\mathbf{\mu g} / \mathbf{m L}\end{array}$ & $\begin{array}{c}\text { SRNL } \\
\text { measured } \\
\left.\text { initial [Na }{ }^{+}\right], \boldsymbol{M}\end{array}$ \\
\hline 6.0 & 6.2308 & 120000 & 5.2174 & 148000 & 6.4376 \\
1.0 & 1.0720 & 17200 & 0.7478 & 29000 & 1.2614 \\
0.1 & 0.0840 & 2190 & 0.0952 & 4180 & 0.1818 \\
0.01 & 0.0094 & 853 & 0.0371 & 575 & 0.0250 \\
0.001 & 0.0009 & 59.8 & 0.0026 & No analysis & No analysis \\
\hline
\end{tabular}


Table 33. Results of $\mathrm{NaOH}-0$ ly titrations

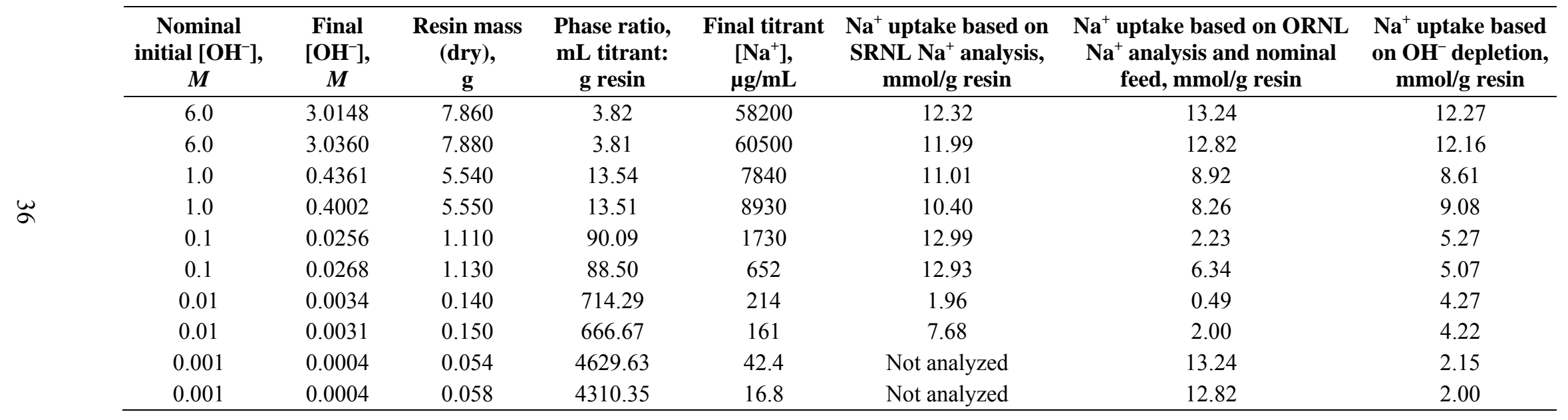




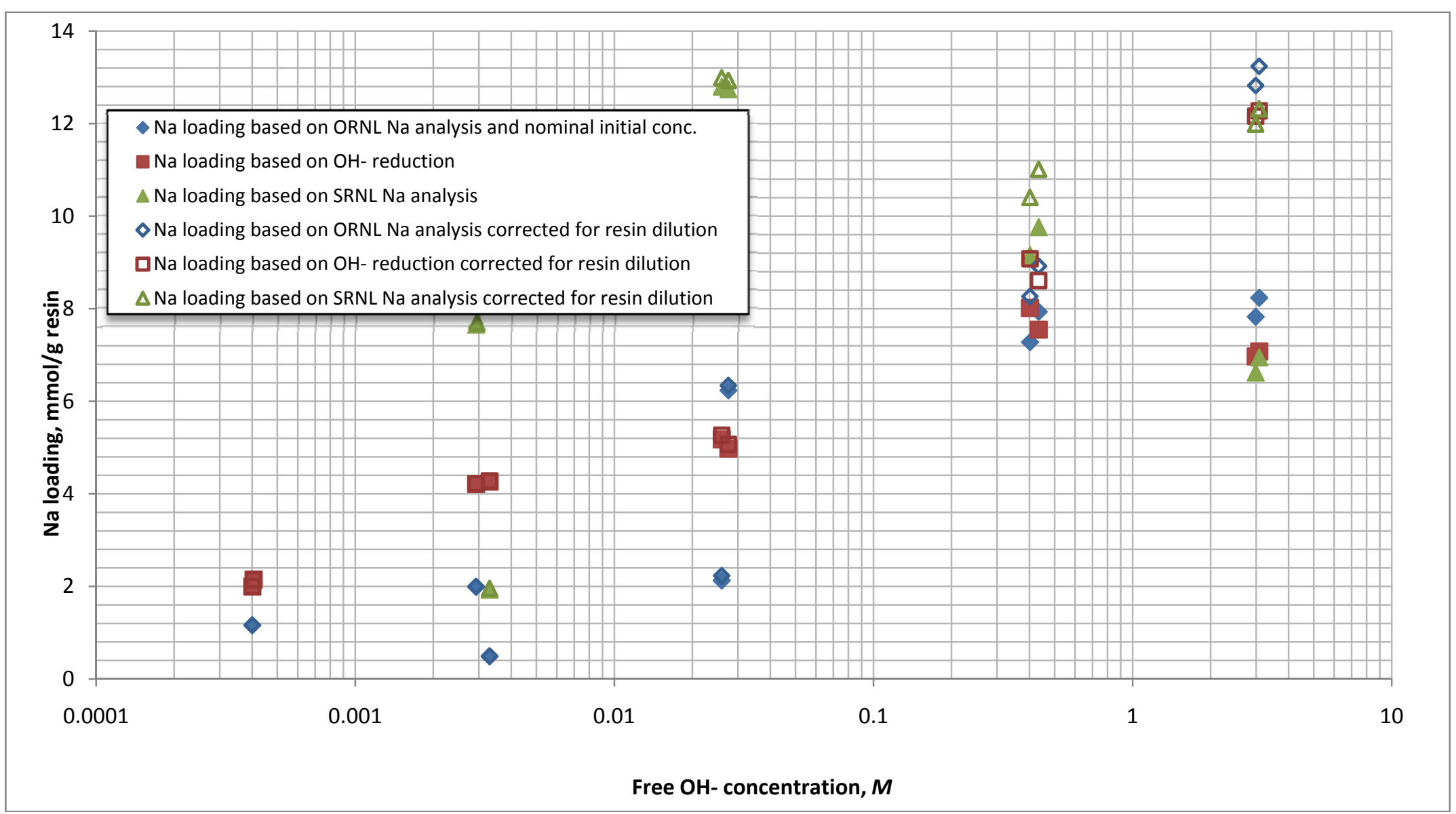

Fig. 14. Results of $\mathrm{NaOH}$-only resin titration. 
As a result of inconsistent ICP-AES results, replicate samples of titrants and raffinates were sent to SRNL for analysis (Table 33). At the two higher initial (titrant) Na concentration conditions, the SRNL analysis results are in closer agreement with target and hydroxide-inferred sodium results than are the ORNL Na analytical results. Similarly, SRNL raffinate results are similar to results from ORNL $\mathrm{OH}^{-}$ determinations at the higher Na condition; consequently, loading results are also similar. However, at the lower Na concentration conditions, both the titrant and raffinate sodium concentrations obtained at SRNL are elevated relative ORNL $\mathrm{Na}^{+}$and $\mathrm{OH}^{-}$results. Because the disparity is between SRNL $\mathrm{Na}^{+}$results and ORNL hydroxide-inferred results is greater for titrants than raffinates, loadings calculated based on the former are higher than those from the latter.

All loading results have been calculated after adjusting the starting solution concentrations for dilution introduced the use of damp resin in the titrations. Results from all analyses are presented in Fig. 14. Values calculated with and without correction for water introduced with the damp resin are plotted to illustrate the effect of resin dilution.

\subsubsection{Titrations with $\mathrm{NaOH} / \mathrm{Neutral}$ Salt $\left(\mathrm{NaNO}_{3}\right)$}

Titrations were performed using solutions containing $\mathrm{NaOH}$ and $\mathrm{NaNO}_{3}$ at the concentrations listed in Table 34. As indicated, all titrants had a nominal total sodium concentration of $6 M$, with the exception of an $\mathrm{NaOH}$-only titration using a nominal feed concentration of 11.5 M. Resin/titrant slurries were agitated as in the previous two tests. Initial and final samples of each titrant solution were collected and analyzed for total sodium. One additional test was also conducted to evaluate potassium uptake under high ionic strength conditions ( $6 \mathrm{M} \mathrm{KOH}$, nominally) for direct comparison to the results with sodium.

Table 34. Nominal compositions for neutral sodium salt tests

\begin{tabular}{cccc}
\hline $\begin{array}{c}\text { Initial } \mathbf{~ N a H} \\
\text { conc., } \boldsymbol{M}\end{array}$ & $\begin{array}{c}\text { Calculated } \\
\text { initial } \mathbf{~ p H}\end{array}$ & $\begin{array}{c}\mathbf{N a N O}_{3} \text { conc., } \\
\boldsymbol{M}\end{array}$ & $\begin{array}{c}\text { Phase ratio } \\
\text { (mL liq:g dry solid) }\end{array}$ \\
\hline 11.5 & 14.8 & 0.0 & 3 \\
11.5 & 14.8 & 0.0 & 4 \\
2.0 & 14.3 & 4.0 & 5 \\
1.0 & 14.0 & 5.0 & 7 \\
0.5 & 13.7 & 5.5 & 13 \\
0.1 & 13.0 & 5.9 & 50 \\
\hline
\end{tabular}

Results from analysis of titrants used in the neutral salt titration are presented in Table 35. As in the previous results, there is significant offset between nominal (target) sodium concentrations and the ICPAES results; as in previous results the analysis values are depressed relative to the target $\mathrm{Na}^{+}$ concentrations and the sodium values that can be inferred from the measured $\mathrm{OH}^{-}$concentrations (where the only intended sodium contribution is from $\mathrm{NaOH}$ ).

Table 35. Initial conditions for titrations using $\mathrm{NaOH}$ and $\mathrm{NaOH} / \mathrm{NaNO}_{3}$ blends

\begin{tabular}{ccccc}
\hline Test no. & $\begin{array}{c}\text { Target }\left[\mathbf{O H}^{-}\right], \\
\boldsymbol{M}\end{array}$ & $\begin{array}{c}\text { Target }\left[\mathbf{N a}^{+}\right], \\
\boldsymbol{M}\end{array}$ & $\begin{array}{c}\text { Measured initial } \\
{\left[\mathbf{O H}^{-}\right], \boldsymbol{M}}\end{array}$ & $\begin{array}{c}\text { Measured } \\
\text { initial }\left[\mathbf{N a}^{+}\right], \boldsymbol{M}\end{array}$ \\
\hline 1 (NaOH only) & 11.50 & 11.50 & 10.8732 & 8.4348 \\
2 & 2.00 & 6.00 & 1.9132 & 5.0000 \\
3 & 1.00 & 6.00 & 0.8757 & 4.3913 \\
4 & 0.50 & 6.00 & 0.3749 & 4.4783 \\
5 & 0.10 & 6.00 & 0.0740 & 4.9130 \\
\hline
\end{tabular}

Results of the neutral salt titrations are presented in Table 36 and Fig. 15. Values in Table 36 have been corrected for resin water dilution; corrected and uncorrected values are presented in Fig. 15. Due to 
Table 36. Results of titrations using $\mathrm{NaOH}$ and $\mathrm{NaOH} / \mathrm{NaNO}_{3}$ blends

\begin{tabular}{|c|c|c|c|c|c|c|c|}
\hline Test no. & $\begin{array}{c}\text { Resin mass } \\
\text { (dry), } \\
\text { g }\end{array}$ & $\begin{array}{c}\text { Titrant } \\
\text { volume, } \\
\text { mL }\end{array}$ & $\begin{array}{l}\text { Phase ratio, } \\
\text { mL titrant: } \\
\text { g resin }\end{array}$ & $\begin{array}{c}\text { Final }\left[\mathrm{OH}^{-}\right] \text {, } \\
\qquad M\end{array}$ & $\begin{array}{l}\text { Final titrant } \\
{\left[\mathrm{Na}^{+}\right], \mu \mathrm{g} / \mathrm{mL}}\end{array}$ & $\begin{array}{l}\mathrm{Na}^{+} \text {uptake based } \\
\text { on } \mathrm{Na}^{+} \text {nominal } \\
\text { feed, } \mathrm{mmol} / \mathrm{g} \text { resin }\end{array}$ & $\begin{array}{c}\mathrm{Na}^{+} \text {uptake based } \\
\text { on } \mathrm{OH}^{-} \text {depletion, } \\
\text { mmol/g resin }\end{array}$ \\
\hline 1 & 8.408 & 25 & 2.9734 & 5.21 & 117000 & 2.85 & 7.68 \\
\hline 1 replicate & 6.329 & 25 & 3.9501 & 6.21 & 175000 & -4.37 & 8.58 \\
\hline 2 & 10.124 & 50 & 4.9388 & 0.29 & 72900 & 4.30 & 6.21 \\
\hline 2 replicate & 10.106 & 50 & 4.9476 & 0.38 & 88700 & 0.91 & 5.77 \\
\hline 3 & 7.272 & 50 & 6.8757 & 0.02 & 92400 & -1.83 & 4.97 \\
\hline 3 replicate & 7.245 & 50 & 6.9013 & 0.02 & 80800 & 1.65 & 5.05 \\
\hline 4 & 5.879 & 75 & 12.7573 & 0.008 & 87500 & 3.78 & 4.28 \\
\hline 4 replicate & 5.869 & 75 & 12.7790 & 0.009 & 103000 & -4.82 & 4.28 \\
\hline 5 & 2.047 & 100 & 48.8520 & 0.001 & 98900 & 24.32 & 3.46 \\
\hline 5 replicate & 2.047 & 100 & 48.8520 & 0.001 & 103000 & 15.61 & 3.46 \\
\hline
\end{tabular}




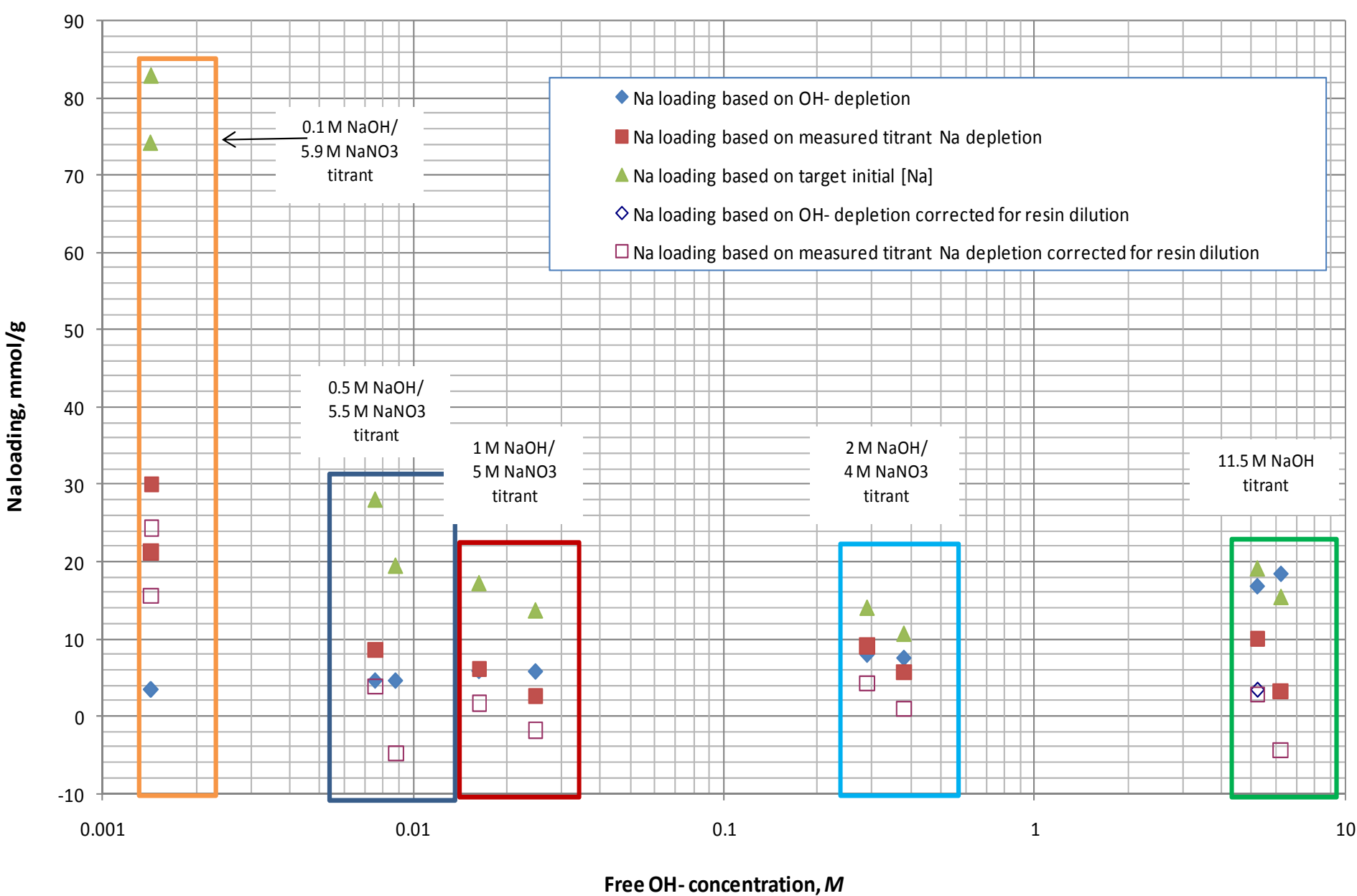

Fig. 15. Results of $\mathrm{NaOH}$ titrations with neutral salt $\left(\mathrm{NaNO}_{3}\right)$. 
the severely depressed initial sodium values obtained by ICP-AES analysis, differences between nominal initial $\mathrm{Na}^{+}$concentration and final, measured values have been used to calculate the loading values listed in next-to-last column. Loadings calculated using nominal and measured initial $\mathrm{Na}^{+}$concentrations, and those inferred from $\mathrm{OH}^{-}$concentrations (i.e., the loading contribution from $\mathrm{NaOH}$ ), are presented in the figure. Results based on $\mathrm{OH}^{-}$depletion alone are presented in Fig. 16, and indicate a high degree of consistency.

Comparison of the $\mathrm{OH}^{-}$based results with $\mathrm{NaOH}$-only titration results (Fig. 14) indicates similar sodium loading contributions from salt/hydroxide blends and hydroxide-only titrants. Relative to the effect of the neutral salt, the magnitude of the increase in adsorption with increasing $\mathrm{OH}^{-}$concentration is minor. Results indicated a relatively small increase in sodium uptake at lower neutral salt concentrations and an exponential increase at concentrations between 5 and $6 \mathrm{M}$.

Hydroxide uptake results for solutions with and without $6 \mathrm{M}$ sodium nitrate background are very similar. It appears that in the concentration of interest $\left(\leq 2.5 \mathrm{M} \mathrm{OH}^{-}\right)$the adsorption of neutral salts does not occur. Furthermore, under these conditions sodium loadings are similar to previously measured values of approximately $6 \mathrm{mmol} / \mathrm{g}$ resin.

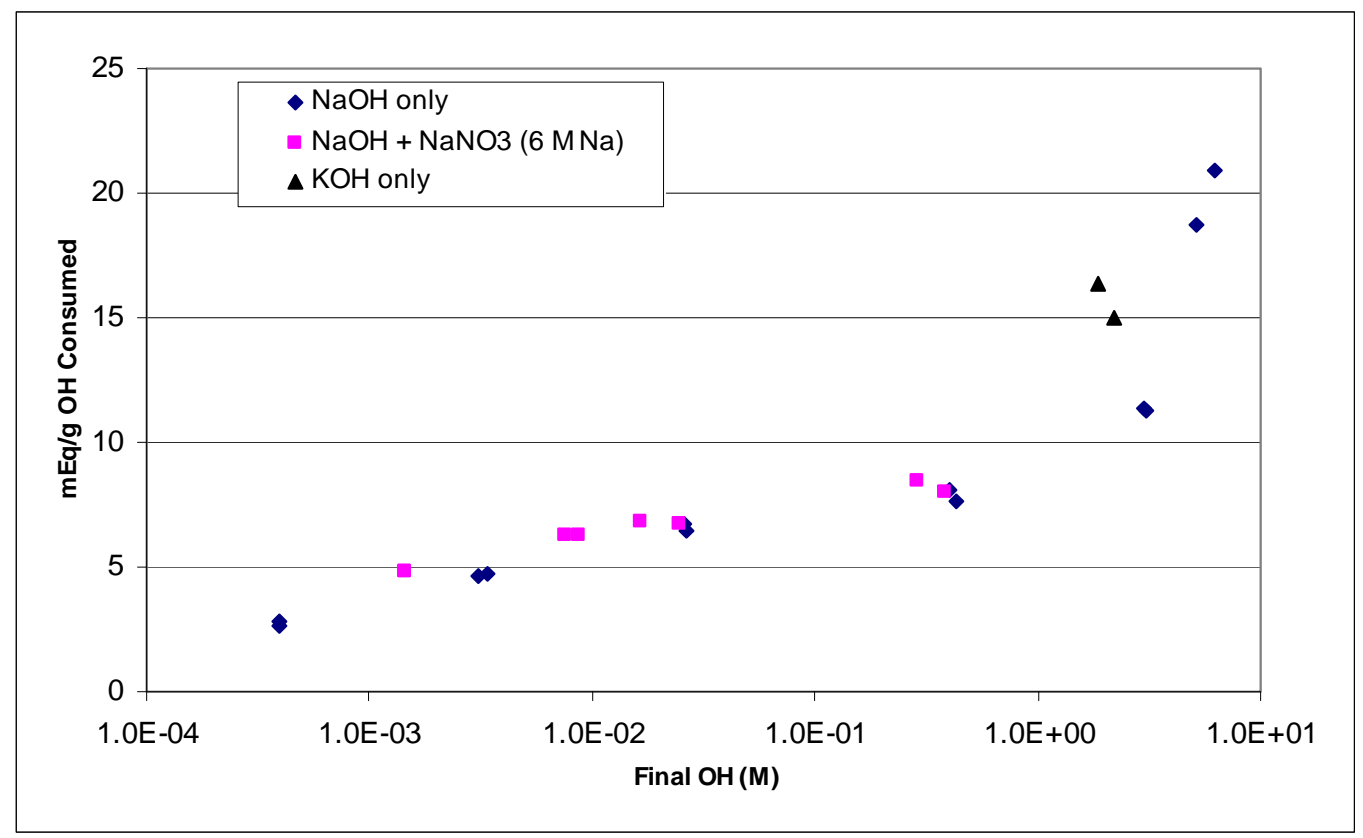

Fig. 16. Sodium loading (expressed as $\mathrm{OH}^{-}$consumed) versus final $\mathrm{OH}^{-}$concentration.

\subsubsection{Potassium Loading Results}

Titrant composition and loading results from a high ionic strength solution of $\mathrm{KOH}$ are presented in Table 37. Comparison of the potassium loading results with the $\mathrm{NaOH}$ test results (Fig. 15) at comparable metal cation concentration are indicative of the selectivity of RF resin for potassium. Hydroxide uptake from a titrant with a $\mathrm{NaOH}$ concentration of $6 M(\sim 11 \mathrm{mmol} / \mathrm{g})$ are lower than the loading observed with a $6 \mathrm{M} \mathrm{KOH}$ solution $(\sim 15 \mathrm{mmol} / \mathrm{g})$. 
Table 37. Conditions and results from high ionic strength test with potassium

\begin{tabular}{|c|c|c|c|c|c|c|c|c|}
\hline $\begin{array}{l}\text { Nominal } \\
\text { initial } \\
{\left[\mathrm{OH}^{-}\right], M}\end{array}$ & $\begin{array}{c}\underset{\text { Nominal }}{\text { initial }\left[K^{+}\right],} \\
M\end{array}$ & $\begin{array}{c}\text { Resin } \\
\text { mass } \\
\text { (dry), g }\end{array}$ & $\begin{array}{c}\text { Titrant } \\
\text { volume, } \\
\text { mL }\end{array}$ & $\begin{array}{l}\text { Phase } \\
\text { ratio, mL } \\
\text { titrant: } \\
\text { g resin }\end{array}$ & $\begin{array}{c}\text { Final } \\
{\left[\mathrm{OH}^{-}\right]} \\
M\end{array}$ & $\begin{array}{c}\text { Final } \\
\text { titrant } \\
{\left[\mathrm{K}^{+}\right]} \\
\mu \mathrm{g} / \mathbf{m L}\end{array}$ & $\begin{array}{c}\mathbf{K}^{+} \text {uptake } \\
\text { based on } \mathbf{K}^{+} \\
\text {nominal feed, } \\
\text { mmol/g resin }\end{array}$ & $\begin{array}{c}\mathrm{K}^{+} \text {uptake } \\
\text { based on } \mathrm{OH}^{-} \\
\text {depletion, } \\
\text { mmol/g resin }\end{array}$ \\
\hline 2 & 6 & 6.352 & 25 & 3.82 & 2.18 & 81500 & 9.96 & 5.26 \\
\hline 2 & 6 & 6.325 & 25 & 3.81 & 1.87 & 85300 & 9.64 & 6.52 \\
\hline
\end{tabular}

\section{CONCLUSIONS}

\subsection{RADIOLYSIS}

RF resin was irradiated under various conditions to determine if the resin degrades when exposed to a radiation field. The irradiated samples produced gas, which was then analyzed for gases and volatile organic compounds. The nitric acid sample from both irradiation facilities (C-60 source and HFIR) had the highest total gas generation. Hydrogen was generated the most while oxygen was consumed by the resin. The amount of hydrogen produced and oxygen consumed increased as the radiation exposure increased.

After irradiation, the resin and liquid were examined for degradation. The resin that was had a known oxygen exposure clumped more and was noticeably darker than the new resin. The liquid was also considerably more discolored when using the known oxygen exposure resin and when exposing the new resin to higher doses. The solutions were analyzed. No standard SVOCs were detected; formaldehyde was only detected in the water sample from the Co-60 source. The discoloration does not seem to have an effect on the resin performance, although it indicates that some sort of degradation is occurring

\subsection{D $_{\mathrm{Cs}}$ DETERMINATIONS}

Cesium partitioning results obtained in the various experiments exhibited considerable internal consistency, both in trends observed and in data collected under similar and identical test conditions. Throughout with simple simulants, $\mathrm{D}_{\mathrm{Cs}}$ values obtained were significantly less than values obtained previously and were therefore below values predicted by the new isotherm. It remains unclear what the source of the discrepancy is; agreement between results obtained from experiments performed at ORNL and SRNL using the same starting materials indicate that the offset does not reflect experimental differences between the two sites. Data collected at SRNL and ORNL using AP-101 is in relatively good agreement with predicted values over a range of cesium concentrations.

Consistent reduction in cesium adsorption with increased equilibration temperature was observed. This result is consistent with previously reported results. Results from extended contact equilibrations exhibit minimal difference from 3-day contacts at equal temperatures.

\subsection{TITRATIONS}

Results of titrations performed using nitrate and chloride salts of sodium indicate no effect of anion species on sodium uptake. Results from $\mathrm{NaOH} /$ neutral salt combinations having equal total initial sodium indicate a slight preference for sodium supplied from the hydroxide, i.e., sodium loading is somewhat higher as a result of contact with $6 \mathrm{M} \mathrm{Na}+$ solutions having increasing proportions of $\mathrm{NaOH}$. However, sodium partition results from $\mathrm{NaOH}$ and $\mathrm{NaOH} / \mathrm{NaNO}_{3}$ solutions having equal total sodium demonstrate high levels of consistency. 


\section{REFERENCES}

Arm, S. T., D. L. Blanchard, Jr., K. P. Brooks, W. C. Buchmiller, B. J. Cook, J. M. Cuta, S. K. Fiskum, Z. Hontz, C. Isackson, A. A. Schepmoes, and D. E. Wallace. Laboratory Scale Hydraulic Testing of Spherical Resorcinol Formaldehyde Ion Exchange Resins. WTP-RPT-142. Battelle-Pacific Northwest Division, Richland, Washington. 2006.

Bibler, J. P., N. E. Bibler, and C. L. Crawford. Year-End Report for UST: Cesium Extraction Testing Project DOE/DT\&E TTP NO SR1-03-20-01. WSRC-RP-94-146. Westinghouse Savannah River Company, Aiken, South Carolina. 1994.

Carlson, C. D., L. A. Bray, S. A. Bryan, J. A. Franz, D. E. Kurath, S. R. Adami, G. N. Brown, J. R. DesChane, R. J. Elovich, J. C. Linehan, W. S. Shaw, and M. R. Telander. Radiation Testing of Organic Ion Exchange Resins. PNL-10767 UC-600. Pacific Northwest Laboratory, Richland, Washington. 1995.

Crawford, C. L., N. E. Bibler., and J. P. Bibler. An Investigation of the Radiolytic Stability of a Resorcinol-Formaldehyde Ion Exchange Resin. WSRC-MS-93-550. Westinghouse Savannah River Company, Aiken, South Carolina. 1993.

Fiskum, S. K., S. T. Arm, W. C. Buchmiller, T. Trang-Le, J. E. Martinez, J. Matyas, M. J. Steele, K. K. Thomas, and D. L. Blanchard, Jr. Comparison Testing of Multiple Spherical ResorcinolFormaldehyde Resins for the River Protection Project-Waste Treatment Plant. WTP-RPT-143. Battelle-Pacific Northwest Division, Richland Washington, 2006.

Nash, C. A. Hanford RPP-WTP Alternate Resin Program - Protocol P1-RF: Spherical Resin Sampling from Containers, Resin Pretreatment, F-Factor, and Resin Loading to Column. SRNL-RPP-200400058. Savannah River National Laboratory, Aiken, South Carolina, July 2004.

Smith, F. G. Modeling of Ion-Exchange for Cesium Removal from Dissolved Saltcake in SRS Tanks 1-3, 37, and 41. WSRC-STI-2007-00315. Washington Savannah River Company, Aiken, South Carolina. 2007.

Taylor, P. A. Hydraulic Permeability of Resorcinol-Formaldehyde Resin. ORNL/TM-2009/293. Oak Ridge National Laboratory, Oak Ridge, Tennessee. December 2009.

Taylor, P. A., and H. L. Johnson. Alternate Methods for Eluting Cesium from Spherical ResorcinolFormaldehyde Resin. ORNL/TM-2008/194. Oak Ridge National Laboratory, Oak Ridge, Tennessee. January 2009. 

APPENDIX A. ANALYTICAL RESULTS FOR GAS SAMPLES FROM RADIOLYSIS TESTS 



\section{UNCLASSIFIED}

LIMS 03.08 .048

05/10/2010 07:52

Project: L MS MPO SCHUH GAS

Customer: L P BURNETT Bldg 9995 MS-8189 (865) 576-9141

\begin{tabular}{llll} 
Matrix & $\begin{array}{l}\text { Chain of } \\
\text { Custody }\end{array}$ & $\begin{array}{l}\text { Work Agreement } \\
\text { Number }\end{array}$ & $\begin{array}{l}\text { Charge } \\
\text { Number }\end{array}$ \\
\hdashline GAS & 117947 & $-8--$ & -1473
\end{tabular}

Sample Description:

Location:

Sampler(s):
Analytical Chemistry organization (Quality Services)

P.o. Box 2009 oak Ridge, TN 378318244 (865) 574-2284

Page 1 of 1

official Report

Lab Smpl Id: A101230502

Cust Smpl Id: Co-60 300 SRS

Proj Mgr: L P BURNETT (865) 576-9141

Lab Comments:

Comment objects:

$\ll<<$ None $\gg>>>$

Rpt Basis: AR Test: GAS-GC (GAS CHROMATOGRAPHY OF GAS SAMPLES)

Test

Analysis Method: Y12 Y50-AC-65-6002

Approver: I L MARSHALL/Chemist

Date Date Date Date

Sampled Received Needed Approved

04/26/2010 07:30 05/03/2010 $15: 19 \quad 05 / 17 / 2010 \quad 23: 59 \quad 05 / 10 / 2010 \quad 07: 52$

Sample Status: APPROVED

Sample Approver: J L MARSHALL/Chemist

Prep Method/Date:

Test Status: APPROVED

HT Deadline: $10 / 23 / 201023: 59$

Date Analyzed: 05/05/2010 00:00

Lab Group: MYSSG1

\begin{tabular}{|c|c|c|c|c|c|c|c|c|c|c|}
\hline \multirow[b]{2}{*}{ Analyte Id } & \multirow[b]{2}{*}{ Analyte Name } & \multicolumn{3}{|c|}{ Customer } & \multirow{2}{*}{$\begin{array}{l}\text { Dilution } \\
\text { Factor }\end{array}$} & \multirow{2}{*}{$\begin{array}{l}\text { Lower } \\
\text { Limit }\end{array}$} & \multirow[b]{2}{*}{ Result } & \multirow{2}{*}{$\begin{array}{l}\text { EPA } \\
\text { Qual }\end{array}$} & \multirow[b]{2}{*}{ Confidence } & \multirow[b]{2}{*}{ Unit } \\
\hline & & HT & Lim & $\mathrm{Fn}$ & & & & & & \\
\hline 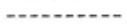 & - & -- & $-\cdot$ & -- & ---- & $-\cdots$ & $\cdots-$ & --- & 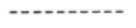 & $-\cdots$ \\
\hline 124389 & Carbon Dioxide ( $\mathrm{CO} 2)$ & & & & 1 & & 0.03 & & & vol $\%$ \\
\hline 630080 & Carbon monoxide & & & & 1 & & 0.24 & & & vol $\%$ \\
\hline 1333740 & Hydrogen, molecular & & & & 1 & & $>6$ & & & vol $\%$ \\
\hline 74828 & Methane & & & & 1 & & 0.017 & & & vol $\%$ \\
\hline 7782447 & oxygen & & & & 1 & & $>5$ & & & vol $\%$ \\
\hline
\end{tabular}

Comment objects:

Test: GAS-MS (Mass Spec. Analysis of Gas by VG 3001)

Rpt Basis: AR Test Req Cnt: 01

Analysis Method: ASO Y/P65-6011

Approver: J L MARSHALL/Chemist

QC Batch/File:

Prep Method/Date:

Test Status: APPROVED

HT Deadline: $10 / 23 / 201023: 59$

Date Ana7yzed: 05/05/2010 00:00

Lab Group: MYSSG1

\begin{tabular}{ll} 
Analyte Id & Analyte Name \\
\hline 7440371 & Argon \\
7440597 & $\begin{array}{l}\text { Helium } \\
1333740\end{array}$ \\
74828 & $\begin{array}{l}\text { Mydrogen, molecular } \\
7727379\end{array}$ \\
7782447 & $\begin{array}{l}\text { Nitrogen, molecular } \\
\text { Oxygen }\end{array}$
\end{tabular}

Customer Dilution Lower HT Lim Fn Factor Limit

Result Qual Confidence unit

1.47 -.- - -

$<0.01 \quad$ vol $\%$

58.67 vol $\%$

Sample Test Comments: $\mathrm{N} 20=8.25 \%[05171205 / 06 / 2010 \quad 10: 46: 02]$

Comment objects: 


\section{UNCLASSIFIED}

LIMS 03.08 .048

05/10/2010 07:51

Project: L MS MPO SCHUH GAS

Customer: L P BURNETT Bldg 9995 MS-8189 (865) 576-9141

\begin{tabular}{|c|c|c|c|c|c|c|c|}
\hline Matrix & $\begin{array}{l}\text { Chain of } \\
\text { Custody }\end{array}$ & $\begin{array}{l}\text { Work Agreement } \\
\text { Number }\end{array}$ & $\begin{array}{l}\text { Charge } \\
\text { Number }\end{array}$ & $\begin{array}{l}\text { Date } \\
\text { Sampled }\end{array}$ & $\begin{array}{l}\text { Date } \\
\text { Received }\end{array}$ & $\begin{array}{l}\text { Date } \\
\text { Needed }\end{array}$ & $\begin{array}{l}\text { Date } \\
\text { Approved }\end{array}$ \\
\hline---- & --- & n-n & $\cdots-$ & -..... & $----\infty$ & ----- & - - n- \\
\hline GAS & 117947 & 81473 & $745 A 0221$ & $04 / 26 / 2010 \quad 07: 30$ & $05 / 03 / 2010 \quad 15: 19$ & $05 / 17 / 2010 \quad 23: 59$ & $05 / 10 / 2010 \quad 07: 51$ \\
\hline & & $\begin{array}{l}\text { cription: } \\
\text {-ocation: } \\
\text { npler(s): }\end{array}$ & & & \multicolumn{3}{|c|}{$\begin{array}{l}\text { Sample Status: APPROVED } \\
\text { Sample Approver: J L MARSHALL/Chemist }\end{array}$} \\
\hline
\end{tabular}

Customer comments:

Lab comments:

Comment objects:
Analytical Chemistry Organization (Quality Services)

P.o. Box 2009 oak Ridge, TN 378318244 (865) 574-2284

Page 1 of 1

official Report

Proj Mgr: L P BURNETT (865) 576-9141
Lab Smp1 Id: A101230500
Rpt Basis: AR

Test Req Cnt: 01

Analysis Method: $\mathrm{Y} 12$ Y50-AC-65-6002

Approver: J L MARSHALL/Chemist QC Batch/File:
Prep Method/Date:

Test Status: APPROVED

HT Deadline: 10/23/2010 23:59

Date Analyzed: 05/05/2010 00:00

Lab Group: MYSSG1

$\begin{array}{ll}\text { Analyte Id } & \text { Analyte Name } \\ 124389 & \text { Carbon Dioxide (CO2) } \\ 630080 & \text { Carbon monoxide } \\ 1333740 & \text { Hydrogen, molecular } \\ 74828 & \text { Methane } \\ 7727379 & \begin{array}{l}\text { Nitrogen, molecular } \\ 7782447\end{array} \\ & \text { Oxygen }\end{array}$

\begin{tabular}{|c|c|c|c|c|c|c|c|c|}
\hline \multicolumn{3}{|c|}{ Customer } & \multirow{2}{*}{$\begin{array}{l}\text { Dilution } \\
\text { Factor }\end{array}$} & \multirow{2}{*}{$\begin{array}{l}\text { Lower } \\
\text { Limit }\end{array}$} & \multirow[b]{2}{*}{ Result } & \multirow{2}{*}{$\begin{array}{l}\text { EPA } \\
\text { Qual }\end{array}$} & \multirow[b]{2}{*}{ Confidence } & \multirow[b]{2}{*}{ Unit } \\
\hline HT & Lim & $\mathrm{Fn}$ & & & & & & \\
\hline-- & $\cdots$ & -- & - - & --- & -n- & $-\cdots$ & - & --- \\
\hline & & & 1 & & 0.007 & & & vol $\%$ \\
\hline & & & 1 & & 0.03 & & & vol $\%$ \\
\hline & & & 1 & & $>5$ & & & vol $\%$ \\
\hline & & & 1 & & 0.005 & & & vol \% \\
\hline & & & 1 & & $>5$ & & & vol \% \\
\hline & & & 1 & & $>1.6$ & & & vol \% \\
\hline
\end{tabular}

Comment objects:

Rpt Basis: AR Test Req Cnt: 01 Analysis Method: ASO Y/P65-6011 Approver: J L MARSHALL/Chemist QC Batch/File:

Test: GAS-MS (Mass Spec. Analysis of Gas by VG 3001)

Prep Method/Date:

Test Status: APPROVED

HT Deadline: $10 / 23 / 2010 \quad 23: 59$

Date Analyzed: 05/05/2010 00:00 Lab Group: MYSSG1

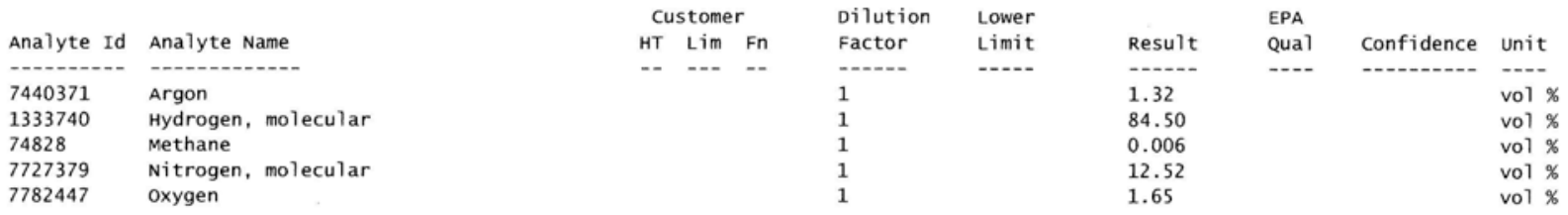

Sample Test Comments: $\mathrm{N} 20=<.01 \%[05171205 / 06 / 201010: 39: 10]$

Comment objects :

सhe results relate only to the items tested.

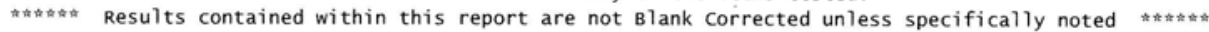

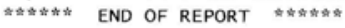

UNCLASSIFIED 


\section{UNCLASSIFIED}

LIMS 03.08 .048

05/10/2010 07:50

Project: L MS MPO SCHUH GAS

Customer: L P BURNETT B1dg 9995 MS-8189 (865) 576-9141

\begin{tabular}{|c|c|c|c|c|c|c|c|}
\hline Matrix & $\begin{array}{l}\text { Chain of } \\
\text { Custody }\end{array}$ & $\begin{array}{l}\text { Work Agreement } \\
\text { Number }\end{array}$ & $\begin{array}{l}\text { Charge } \\
\text { Number }\end{array}$ & $\begin{array}{l}\text { Date } \\
\text { Sampled }\end{array}$ & $\begin{array}{l}\text { Date } \\
\text { Received }\end{array}$ & $\begin{array}{l}\text { Date } \\
\text { Needed }\end{array}$ & $\begin{array}{l}\text { Date } \\
\text { Approved }\end{array}$ \\
\hline -..... & n....... & - n-.- & - & - - & - & - - & - \\
\hline GAS & 117947 & 81473 & 745A0221 & $04 / 26 / 2010 \quad 07: 30$ & $05 / 03 / 2010 \quad 15: 19$ & $05 / 17 / 2010 \quad 23: 59$ & $05 / 10 / 2010 \quad 07: 50$ \\
\hline
\end{tabular}

Customer comments:

Lab comments:

Comment objects:
Analytical Chemistry organization (Quality Services)

P.O. Box 2009 oak Ridge, TN 378318244 (865) 574-2284

Proj Mgr: L P BURNETT (865) 576-9141 official Report
Page 1 of 1

Lab Smp1 Id: A101230501

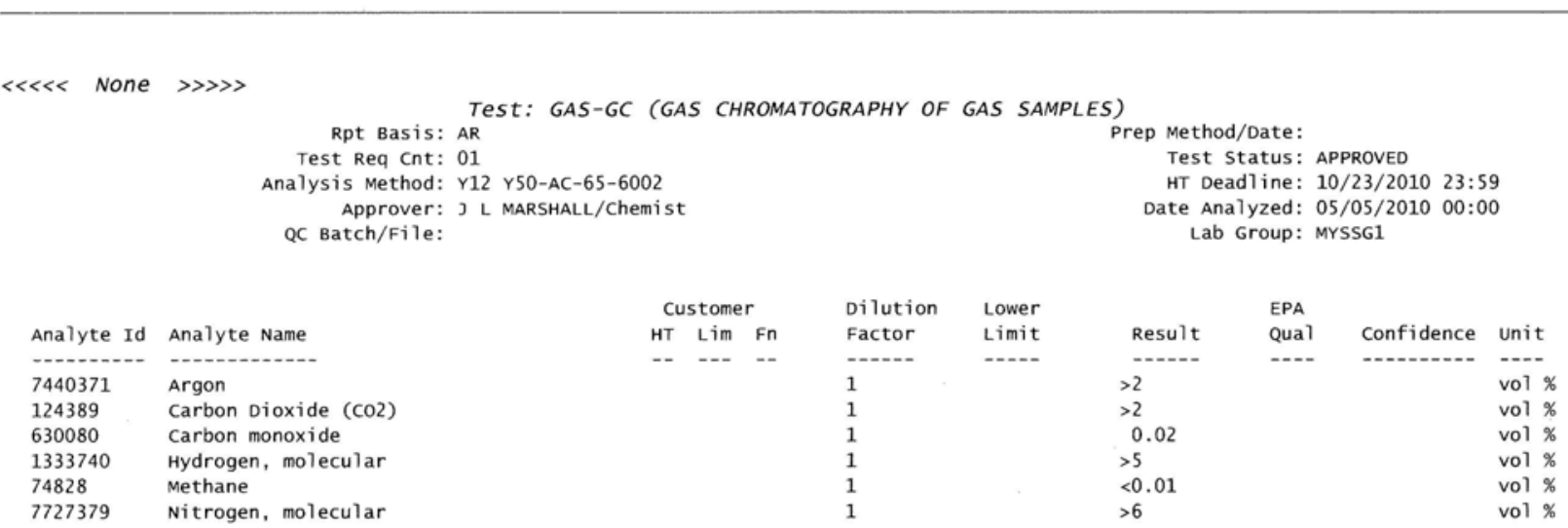

comment objects: Test: GAS-MS (Mass Spec. Analysis of Gas by VG 3001)

Rpt Basis: AR Test Req Cnt: 01

Analysis Method: ASO Y/P65-6011

Approver: J L MARSHALL/Chemist

QC Batch/File:

Test Status: APPROVED

HT Deadline: 10/23/2010 23:59

Date Analyzed: 05/05/2010 00:00

Lab Group: MYSSG1

$\begin{array}{ll}\text { Analyte Id } & \text { Analyte Name } \\ 7440371 & \text { Argon } \\ 124389 & \text { Carbon Dioxide }(\mathrm{CO} 2) \\ 7440597 & \text { Helium } \\ 1333740 & \text { Hydrogen, molecular } \\ 74828 & \text { Methane } \\ 7727379 & \text { Nitrogen, molecular } \\ 7782447 & \text { oxygen }\end{array}$

\begin{tabular}{|c|c|c|c|c|c|c|c|c|}
\hline \multicolumn{3}{|c|}{ Customer } & \multirow{2}{*}{$\begin{array}{l}\text { Dilution } \\
\text { Factor }\end{array}$} & \multirow{2}{*}{$\begin{array}{l}\text { Lower } \\
\text { Limit }\end{array}$} & & \multirow{2}{*}{$\begin{array}{l}\text { EPA } \\
\text { Qua1 }\end{array}$} & \multirow[b]{2}{*}{ Confidence } & \multirow[b]{2}{*}{ Unit } \\
\hline HT & Lim & Fn & & & Result & & & \\
\hline- & $\cdots$ & $\cdots$ & - n-s- & $\cdots$ & - n-s. & $\cdots$ & - & $\cdots$ \\
\hline & & & 1 & & 2.38 & & & vol \% \\
\hline & & & 1 & & 33.7 & & & vol \% \\
\hline & & & 1 & & $<0.01$ & & & vol \% \\
\hline & & & 1 & & 29.28 & & & vol $\%$ \\
\hline & & & 1 & & $<0.01$ & & & vol \% \\
\hline & & & 1 & & 30.70 & & & vol \% \\
\hline & & & 1 & & 0.28 & & & vol \% \\
\hline
\end{tabular}

Sample Test Comments: $\mathrm{N} 20=3.65 \%[051712 \quad 05 / 06 / 2010 \quad 10: 42: 41]$

Comment objects:

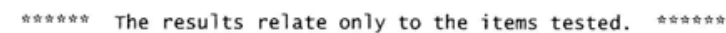

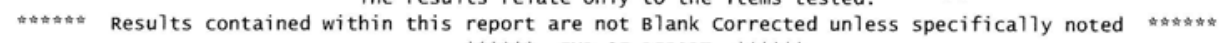

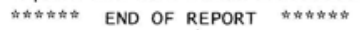

UNCLASSIFIED 


\section{UNCLASSIFIED}

LIMS 03.08 .048

03/08/2010 13:36
Analytical Chemistry organization (Quality Services)

Page 1 of 1
Lab Smp1 Id: A100550035

official Report

Cust Smpl Id: Co60 source 250 Mrad SRS

Project: L MS MPO SCHUH GAS

Proj Mgr: L. P BURNETT (865) 576-9141

Customer: L P BURNETT Bldg 9995 MS-8189 (865) 576-9141

\begin{tabular}{|c|c|c|c|c|c|c|c|}
\hline Matrix & $\begin{array}{l}\text { Chain of } \\
\text { Custody }\end{array}$ & $\begin{array}{l}\text { work Agreement } \\
\text { Number }\end{array}$ & $\begin{array}{l}\text { Charge } \\
\text { Number }\end{array}$ & $\begin{array}{l}\text { Date } \\
\text { Sampled }\end{array}$ & $\begin{array}{l}\text { Date } \\
\text { Received }\end{array}$ & $\begin{array}{l}\text { Date } \\
\text { Needed }\end{array}$ & $\begin{array}{l}\text { Date } \\
\text { Approved }\end{array}$ \\
\hline$-\cdots-$ & - - & --- & - - & $\cdots$ & - n- & $-\cdots$ & --n-s-- \\
\hline GAS & 117935 & 81473 & $745 A 0221$ & 02/23/2010 00:00 & $02 / 23 / 2010 \quad 14: 30$ & 03/09/2010 23:59 & 03/08/2010 13:36 \\
\hline
\end{tabular}

Customer Comments:

Lab comments:

Comment objects:

$\ll<<$ None $\gg \gg>>$

Rpt Basis: $A R$ Test: GAS-GC (GAS CHROMATOGRAPHY OF GAS SAMPLES) Test Req Cnt: 01 Analysis Method: Y12 Y59-65-6002 Approver: J L MARSHALL/Chemist QC Batch/File:

Prep Method/Date:

Test Status: APPROVED

HT Deadline: 08/22/2010 23:59 Date Analyzed: 03/04/2010 00:00

Lab Group: MYSSG1

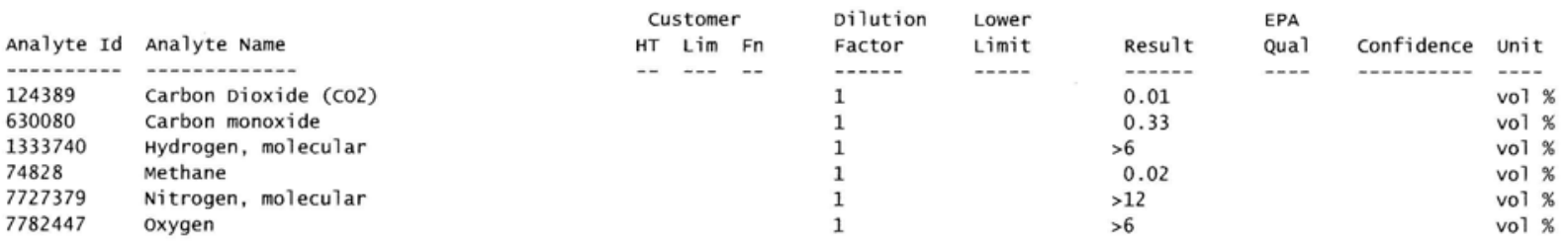

Comment objects:

Rpt Basis: AR

Test: GAS-MS (Mass Spec. Ana7ysis of Gas by VG 3001) Rpt Basis: AR
Test Req Cnt: 01

Analysis Method: ASO Y/P65-6011

Approver: J L MARSHALL/Chemist

QC Batch/File:

Prep Method/Date:

Test Status: APPROVED

HT Deadline: 08/22/2010 23:59

Date Analyzed: 03/04/2010 00:00

Lab Group: MYSSG1

\begin{tabular}{|c|c|c|c|c|c|c|c|c|c|c|}
\hline \multirow{3}{*}{ Analyte Id } & \multirow[b]{2}{*}{ Analyte Name } & \multicolumn{3}{|c|}{ Customer } & \multirow{2}{*}{$\begin{array}{l}\text { Dilution } \\
\text { Factor }\end{array}$} & \multirow{2}{*}{$\begin{array}{l}\text { Lower } \\
\text { Limit }\end{array}$} & \multirow[b]{2}{*}{ Result } & \multirow{2}{*}{$\begin{array}{l}\text { EPA } \\
\text { Qual }\end{array}$} & \multirow[b]{2}{*}{ Confidence } & \multirow[b]{2}{*}{ Unit } \\
\hline & & HT & Lim & $\mathrm{Fn}$ & & & & & & \\
\hline & - & -- & -- & -- & $-\cdots-1$ & ---- & $-\cdots--$ & --- & - n-n- & --- \\
\hline 7440371 & Argon & & & & 1 & & 3.75 & & & vol $\%$ \\
\hline 7440597 & Helium & & & & 1 & & $<0.1$ & & & vol $\%$ \\
\hline 1333740 & Hydrogen, molecular & & & & 1 & & 15.13 & & & vol $\%$ \\
\hline 74828 & Methane & & & & 1 & & 0.02 & & & vol $\%$ \\
\hline 7727379 & Nitrogen, molecular & & & & 1 & & 63.19 & & & vol $\%$ \\
\hline 7782447 & oxygen & & & & 1 & & 13.87 & & & vol $\%$ \\
\hline
\end{tabular}

Sample Test Comments: $\mathrm{N} 2 \mathrm{O}+\mathrm{CO} 2=4.02 \%\left[\begin{array}{lll}018053 & 03 / 08 / 2010 & 13: 25: 30\end{array}\right]$

Comment objects:

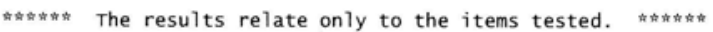

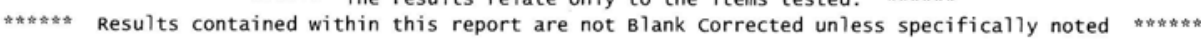

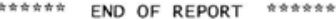

\section{UNCLASSIFIED}




\section{UNCLASSIFIED}

LIMS 03.08 .048

03/08/2010 13:34

Project: L MS MPO SCHUH GAS

\begin{tabular}{|c|c|c|c|c|c|c|c|}
\hline Matrix & $\begin{array}{l}\text { Chain of } \\
\text { Custody }\end{array}$ & $\begin{array}{l}\text { Work Agreement } \\
\text { Number }\end{array}$ & $\begin{array}{l}\text { Charge } \\
\text { Number }\end{array}$ & $\begin{array}{l}\text { Date } \\
\text { Sampled }\end{array}$ & $\begin{array}{l}\text { Date } \\
\text { Received }\end{array}$ & $\begin{array}{l}\text { Date } \\
\text { Needed }\end{array}$ & $\begin{array}{l}\text { Date } \\
\text { Approved }\end{array}$ \\
\hline -...- & - - & - & -..... & -...-- & --n-n- & -no & - - \\
\hline GAS & 117935 & 81473 & 745A0221 & $02 / 23 / 201000: 00$ & $02 / 23 / 2010 \quad 14: 30$ & $03 / 09 / 2010 \quad 23: 59$ & 03/08/2010 13:34 \\
\hline
\end{tabular}

Customer Comments:

Lab Comments:

Comment objects:

PROj Mgr: L P BURNETT (865) 576-9141
Analytical Chemistry Organization (Quality Services)

P.O. Box 2009 oak Ridge, TN 378318244 (865) 574-2284

Page 1 of 1

Cust Smpl Id: Co60 source $250 \mathrm{H} 2 \mathrm{O}$
Lab Smp 1 Id: A100550032

$\ll<<$ None $\gg \gg>$

Rpt Basis: AR

Test: GAS-GC (GAS CHROMATOGRAPHY OF GAS SAMPLES)

Test Req Cnt: 01

Analysis Method: Y12 Y59-65-6002

Approver: J L MARSHALL/Chemist

QC Batch/File:

Prep Method/Date:

Test Status: APPROVED

HT Deadline: 08/22/2010 23:59

Date Analyzed: 03/04/2010 00:00

Lab Group: MYSSG1

\begin{tabular}{|c|c|c|c|c|c|c|c|c|}
\hline \multicolumn{3}{|c|}{ Customer } & \multirow{2}{*}{$\begin{array}{l}\text { Dilution } \\
\text { Factor }\end{array}$} & \multirow{2}{*}{$\begin{array}{l}\text { Lower } \\
\text { Limit }\end{array}$} & \multirow[b]{2}{*}{ Result } & \multirow{2}{*}{$\begin{array}{l}\text { EPA } \\
\text { Qua] }\end{array}$} & \multirow[b]{2}{*}{ Confidence } & \multirow[b]{2}{*}{ Unit } \\
\hline HT & Lim & $\mathrm{Fn}$ & & & & & & \\
\hline -- & -- & -- & --- & --.-- & --.-- & 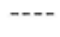 & - & - - \\
\hline & & & 1 & & $>2$ & & & vol $\%$ \\
\hline & & & 1 & & 0.03 & & & vol $\%$ \\
\hline & & & 1 & & 0.03 & & & vol $\%$ \\
\hline & & & 1 & & $>17$ & & & vol \% \\
\hline & & & 1 & & 0.004 & & & vol $\%$ \\
\hline & & & 1 & & $>17$ & & & vol $\%$ \\
\hline
\end{tabular}

Comment objects:

Test: GAS-MS (Mass Spec. Analysis of Gas by VG 3001) Rpt Basis: AR Test Req Cnt: 01 Analysis Method: ASO $\mathrm{Y} / \mathrm{P} 65-6011$ QC Batch/File:

HT Deadline: 08/22/2010 23:59 Date Analyzed: 03/04/2010 00:00 Lab Group: MYSSG1

\begin{tabular}{|c|c|}
\hline Analyte Id & Analyte Name \\
\hline 7440371 & Araon \\
\hline 7440597 & Helium \\
\hline 1333740 & Hydrogen, molecular \\
\hline 74828 & Methane \\
\hline 7727379 & Nitrogen, molecular \\
\hline 7782447 & oxygen \\
\hline
\end{tabular}

\begin{tabular}{|c|c|c|c|c|c|c|c|c|}
\hline \multicolumn{3}{|c|}{ Customer } & \multirow{2}{*}{$\begin{array}{l}\text { Dilution } \\
\text { Factor }\end{array}$} & \multirow{2}{*}{$\begin{array}{l}\text { Lower } \\
\text { Limit }\end{array}$} & & EPA & \multirow[b]{2}{*}{ Confidence } & \multirow[b]{2}{*}{ Unit } \\
\hline HT & Lim & $\mathrm{Fn}$ & & & Result & Qual & & \\
\hline-- & $\cdots$ & -- & -....- & $\ldots$ & $-\ldots$ & $\cdots$ & 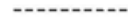 & $-\cdots$ \\
\hline & & & 1 & & 2.65 & & & vol $\%$ \\
\hline & & & 1 & & $<0.1$ & & & vol $\%$ \\
\hline & & & 1 & & 72.59 & & & vol $\%$ \\
\hline & & & 1 & & 0.007 & & & vol \% \\
\hline & & & 1 & & 23.23 & & & vol \% \\
\hline & & & 1 & & 0.99 & & & vol \% \\
\hline
\end{tabular}

Sample Test Comments: $\mathrm{N} 2 \mathrm{O}+\mathrm{CO}=.05 \%[018053 \quad 03 / 08 / 2010 \quad 13: 22: 02]$

Comment objects:

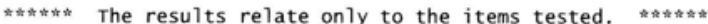

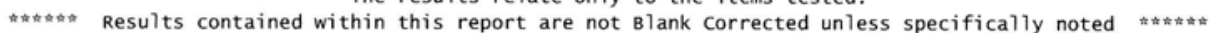

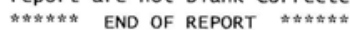

UNCLASSIFIED 


\section{UNCLASSIFIED}

LIMS 03.08 .048

03/08/2010 13:32

Project: L MS MPO SCHUH GAS

Customer: L P BURNETT Bldg 9995 MS-8189 (865) 576-9141

\begin{tabular}{|c|c|c|c|}
\hline Matrix & $\begin{array}{l}\text { Chain of } \\
\text { Custody }\end{array}$ & $\begin{array}{l}\text { Work Agreement } \\
\text { Number }\end{array}$ & $\begin{array}{l}\text { Charge } \\
\text { Number }\end{array}$ \\
\hline ----- & -.... & - n-..- & - \\
\hline GAS & 117935 & 81473 & $745 A 0221$ \\
\hline
\end{tabular}

Analytical Chemistry organization (Quality Services)

P.o. Box 2009 oak Ridge, TN 378318244 (865) 574-2284

official Report
Page 1 of 1

Lab Smpl Id: A100550031

Customer comments:

Lab Comments:

comment objects:

$\ll<<$ None $\gg \gg>$

Rpt Basis: AR Rpt Basis: AR
Test Req Cnt: 01

Analysis Method: Y12 Y59-65-6002

Approver: J L MARSHALL/Chemist

Proj Mgr: L P BURNETT (865) 576-9141

Cust Smp1 Id: Co60 source $250 \mathrm{Mrad}$

QC Batch/File:

Date Date Date Date

Sampled Received Needed Approved

02/23/2010 00:00 02/23/2010 14:30 03/09/2010 23:59 03/08/2010 13:32

Sample Status: APPROVED

Sample Approver: J L MARSHALL/Chemist

\begin{tabular}{|c|c|c|c|c|c|c|c|c|c|}
\hline \multirow{4}{*}{ Analyte Id } & \multirow{3}{*}{$\begin{array}{l}\text { Rpt Basis: } \\
\text { Test Req Cnt: } \\
\text { Analysis Method: } \\
\text { Approver: } \\
\text { QC Batch/File: } \\
\text { Analyte Name }\end{array}$} & $\begin{array}{l}\text { Test: GAS-GC (GAS } \\
\text { AR } \\
01 \\
\text { Y12 Y } 59-65-6002 \\
\text { J L MARSHALL/Chemist }\end{array}$ & S CHROM & \multicolumn{2}{|c|}{ GAS SAT } & $\begin{array}{r}\text { Prep Metl } \\
\text { Test } \\
\text { HT } \\
\text { Date } \\
\text { Le }\end{array}$ & $\begin{array}{l}\text { Date: } \\
\text { atus: A } \\
\text { line: } 0 \\
\text { yzed: } 0 \\
\text { roup: } M\end{array}$ & 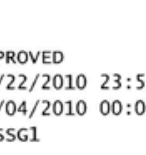 & \\
\hline & & & stomer & Dilution & Lower & & EPA & & \\
\hline & & HT & Lim Fn & Factor & Limit & Result & Qual & Confidence & Unit \\
\hline & - & -- & $---\cdots$ & $m--$ & --- & $-\cdots$ & $\cdots$ & - & --- \\
\hline 7440371 & Argon & & & 1 & & $>0.4$ & & & vol \% \\
\hline 124389 & Carbon Dioxide $(\mathrm{CO} 2)$ & & & 1 & & $>0.8$ & & & vol $\%$ \\
\hline 630080 & Carbon monoxide & & & 1 & & 0.06 & & & vol \% \\
\hline 1333740 & Hydrogen, molecular & & & 1 & & $>1.7$ & & & vol $\%$ \\
\hline 74828 & Methane & & & 1 & & $<0.01$ & & & vol $\%$ \\
\hline 7727379 & Nitrogen, molecular & & & 1 & & $>5$ & & & vol \% \\
\hline
\end{tabular}

Comment objects:

Test: GAS-MS (Mass Spec. Analysis of Gas by VG 3001)

Rpt Basis: AR Test Req Cnt: 01

Analysis Method: ASO Y/P65-6011

Approver: I L MARSHALL/Chemist

QC Batch/File:

Test Status: APPROVED

HT Deadline: $08 / 22 / 201023: 59$

Date Analyzed: 03/04/2010 00:00

Lab Group: MYSSG1

\begin{tabular}{ll} 
Analyte Id & Analyte Name \\
\hline 7440371 & Argon \\
7440597 & Helium \\
1333740 & Hydrogen, molecular \\
74828 & Methane \\
7727379 & Nitrogen, molecular \\
7782447 & oxygen
\end{tabular}

\begin{tabular}{|c|c|c|c|c|}
\hline \multicolumn{3}{|c|}{ Customer } & \multirow{2}{*}{$\begin{array}{l}\text { Dilution } \\
\text { Factor }\end{array}$} & \multirow{2}{*}{$\begin{array}{l}\text { Lower } \\
\text { Limit }\end{array}$} \\
\hline HT & Lim & $\mathrm{Fn}$ & & \\
\hline-- & $\cdots$ & -- & $-\cdots$ & $-\cdots$ \\
\hline & & & 1 & \\
\hline & & & 1 & \\
\hline & & & 1 & \\
\hline & & & 1 & \\
\hline & & & 1 & \\
\hline & & & 1 & \\
\hline
\end{tabular}

$\begin{array}{llll}\text { EPA } & \text { EPA } & & \\ \text { Rual } & \text { Confidence } & \text { Unit } \\ 4.78 & - & & \text { vol } \% \\ 0.1 & & & \text { vol } \% \\ 17.2 & & & \text { vol } \% \\ 0.007 & & & \text { vol } \% \\ 32.16 & & & \text { vol } \% \\ 0.29 & & & \text { vol } \%\end{array}$

Comment objects:

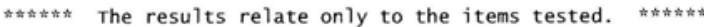

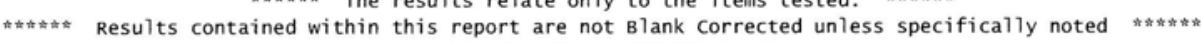

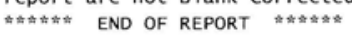

UNCLASSIFIED 


\section{UNCLASSIFIED}

LIMS 03.08 .048

02/17/2010 12:26

Project: L MS MPO SCHUH GAS

Customer: L P BURNETT Bldg 9995 MS-8189 (865) 576-9141

\begin{tabular}{|c|c|c|c|}
\hline Matrix & $\begin{array}{l}\text { Chain of } \\
\text { Custody }\end{array}$ & $\begin{array}{l}\text { Work Agreement } \\
\text { Number }\end{array}$ & $\begin{array}{l}\text { Charge } \\
\text { Number }\end{array}$ \\
\hline GAS & 117931 & 81473 & $745 \mathrm{~A} 0221$ \\
\hline
\end{tabular}

Sampled

01/21/2010 00:00 \begin{tabular}{lll}
$\begin{array}{l}\text { Date } \\
\text { Received }\end{array}$ & $\begin{array}{l}\text { Date } \\
\text { Needed }\end{array}$ & $\begin{array}{l}\text { Date } \\
\text { Approved }\end{array}$ \\
\hdashline $01 / 21 / 2010 \quad 14: 30$ & $-02 / 04 / 2010 \quad 23: 59$ & $-02 / 17 / 2010 \quad 12: 26$
\end{tabular}

Sample status: APPROVED

Sample Approver: J L MARSHALL/Chemist
Page 1 of 1

Lab Smp1 Id: A100250138

Cust Smp 1 Id: Co-60 source 200MradSRSsm

Proj Mgr: L P BURNETT (865) 576-9141

Customer comments:

Lab comments:

Comment objects:

$\ll<<$ None $\gg \gg>$ Test: GAS-GC (GAS CHROMATOGRAPHY OF GAS SAMPLES)

Rpt Basis: AR Test Req Cnt: 01

Analysis Method: Y12 Y59-65-6002

Approver: J L MARSHALL/Chemist

QC Batch/File:

Prep Method/Date:

Test Status: APPROVED

HT Deadline: 07/20/2010 23:59

Date Analyzed: 01/26/2010 00:00

Lab Group: MYSSG1

Analyte Id Analyte Name

Customer Dilution Lower

Analyte Id Analyte Name

-. -.. -.

Factor Limit

Result

Qua?

Confidence unit

Sample Test Comments: No gas in this section of sample tube. [018053 02/17/2010 10:11:58]

Comment objects:

Rpt Basis: $A R$ Test Req Cnt: 01 Analysis Method: ASO Y/P65-6011

Approver: J L MARSHALL/Chemist QC Batch/File:

Test: GAS-MS (Mass Spec. Analysis of Gas by VG 3001)

Prep Method/Date:

Test Status: APPROVED

HT Deadline: 07/20/2010 23:59

Date Analyzed: 01/26/2010 00:00

Lab Group: MYSSG1

\begin{tabular}{|c|c|c|c|c|c|c|c|c|}
\hline \multicolumn{3}{|c|}{ Customer } & \multirow{2}{*}{$\begin{array}{l}\text { Dilution } \\
\text { Factor }\end{array}$} & \multirow{2}{*}{$\begin{array}{l}\text { Lower } \\
\text { Limit }\end{array}$} & \multirow[b]{2}{*}{ Result } & \multirow{2}{*}{$\begin{array}{l}\text { EPA } \\
\text { Qua] }\end{array}$} & \multirow[b]{2}{*}{ Confidence } & \multirow[b]{2}{*}{ Unit } \\
\hline HT & Lim & $\mathrm{Fn}$ & & & & & & \\
\hline-- & $\cdots$ & -- & -....- & $\cdots$ & -....- &.-- & - & --- \\
\hline & & & 1 & & 19.46 & & & vol $\%$ \\
\hline & & & 1 & & $<0.1$ & & & vol \% \\
\hline & & & 1 & & 7.55 & & & vol \% \\
\hline & & & 1 & & $<0.01$ & & & vol $\%$ \\
\hline & & & 1 & & 52.71 & & & vol \% \\
\hline & & & 1 & & 18.73 & & & vol \% \\
\hline
\end{tabular}

Sample Test Comments: $\mathrm{N} 20=1.53 \%[018053 \quad 02 / 17 / 2010 \quad 10: 10: 28]$

Comment objects:

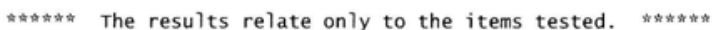

Results contained within this report are not Blank Corrected unless specifically noted

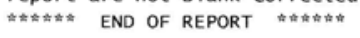

UNCLASSIFIED 


\section{UNCLASSIFIED}

LIMS 03.08 .048

$02 / 17 / 201012: 28$

Project: L MS MPO SCHUH GAS

Customer: L P BURNETT B Bdg 9995 MS-8189 (865) 576-9141

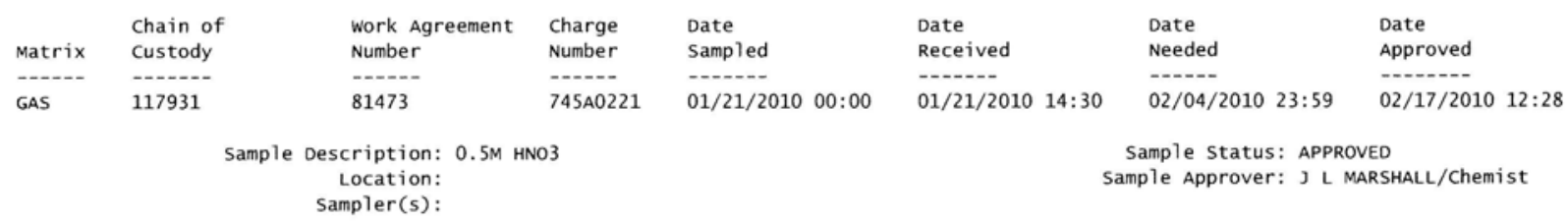

Customer comments:

Lab comments:

Comment objects:
Analytical Chemistry organization (Quality Services)

P.o. Box 2009 oak Ridge, TN 378318244 (865) 574-2284

official Report

Lab Smp1 Id: A100250140

Proj Mgr: L P BURNETT (865) 576-9141 Nifríc
Page 1 of 1

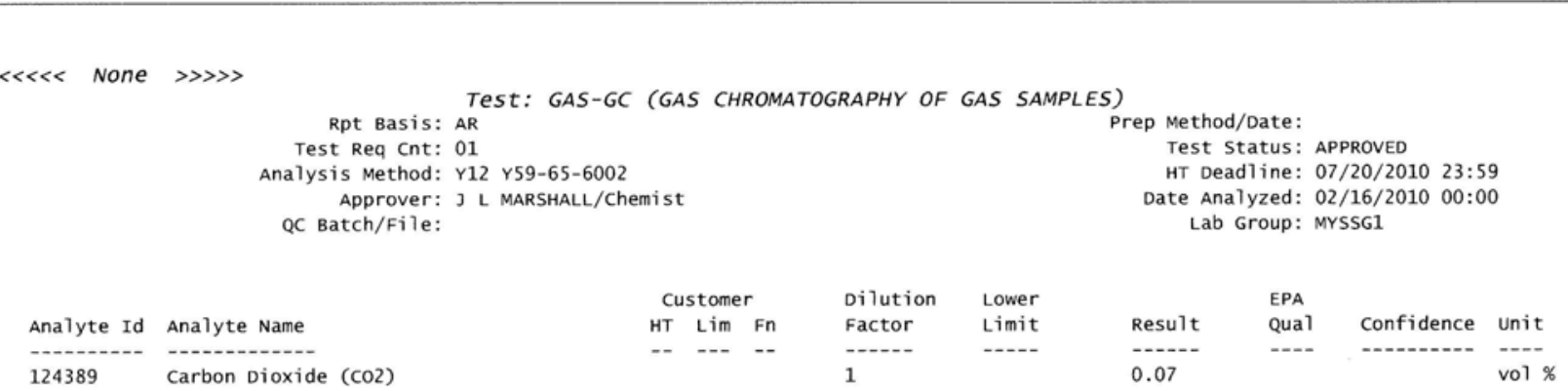

Sample Test Comments: This section of sample contained air. $02>10 \%$ : N2 >39\% [018053 02/17/2010 10:21:26]

comment objects:

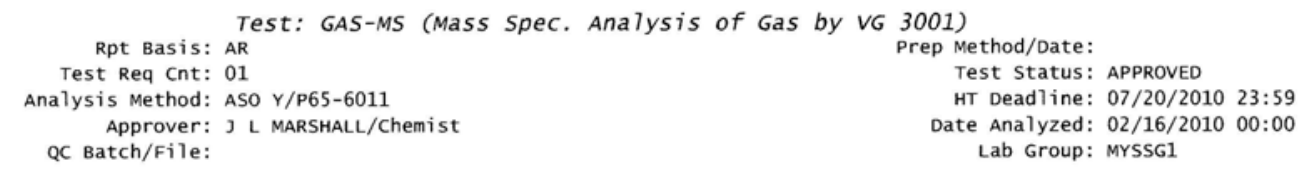

\begin{tabular}{|c|c|c|c|c|c|c|c|c|c|c|}
\hline \multirow[b]{2}{*}{ Analyte Id } & \multirow[b]{2}{*}{ Analyte Name } & \multicolumn{3}{|c|}{ Customer } & \multirow{2}{*}{$\begin{array}{l}\text { Dilution } \\
\text { Factor }\end{array}$} & \multirow{2}{*}{$\begin{array}{l}\text { Lower } \\
\text { Limit }\end{array}$} & \multirow[b]{2}{*}{ Result } & \multirow{2}{*}{$\begin{array}{l}\text { EPA } \\
\text { Qual }\end{array}$} & \multirow[b]{2}{*}{ Confidence } & \multirow[b]{2}{*}{ Unit } \\
\hline & & HT & Lim & $\mathrm{Fn}$ & & & & & & \\
\hline - & - & -- & --- & -- & ---- & $-\cdots$ & $---\overline{-}$ & $-\cdots$ & - & $--\cdot$ \\
\hline 7440371 & Argon & & & & 1 & & 8.9 & & & vol \% \\
\hline 7440597 & Helium & & & & 1 & & $<0.1$ & & & vol \% \\
\hline 1333740 & Hydrogen, molecular & & & & 1 & & 5.16 & & & vol $\%$ \\
\hline 74828 & Methane & & & & 1 & & $<0.01$ & & & vol \% \\
\hline 7727379 & Nitrogen, molecular & & & & 1 & & 45.07 & & & vol \% \\
\hline 7782447 & oxygen & & & & 1 & & 7.3 & & & vol \% \\
\hline
\end{tabular}

Sample Test Comments: $\mathrm{N} 20=33.52 \%[018053 \quad 02 / 17 / 2010 \quad 10: 20: 19]$

comment objects:

क*

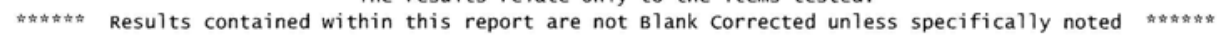

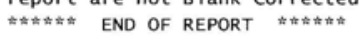

UNCLASSIFIED 


\section{UNCLASSIFIED}

LIMS 03.08 .048

12/09/2009 13:09

Project: L MS MPO SCHUH GAS

Customer: L P BURNETT Bldg 9995 MS-8189 (865) 576-9141

\begin{tabular}{|c|c|c|c|c|c|c|c|}
\hline Matrix & $\begin{array}{l}\text { Chain of } \\
\text { Custody }\end{array}$ & $\begin{array}{l}\text { Work Agreement } \\
\text { Number }\end{array}$ & $\begin{array}{l}\text { Charge } \\
\text { Number }\end{array}$ & $\begin{array}{l}\text { Date } \\
\text { Sampled }\end{array}$ & $\begin{array}{l}\text { Date } \\
\text { Received }\end{array}$ & $\begin{array}{l}\text { Date } \\
\text { Needed }\end{array}$ & $\begin{array}{l}\text { Date } \\
\text { Approved }\end{array}$ \\
\hline GAS & 117882 & 81473 & 745A0221 & $11 / 10 / 200900: 00$ & $11 / 10 / 200915: 30$ & $12 / 09 / 2009 \quad 23: 59$ & $12 / 09 / 2009 \quad 13: 09$ \\
\hline
\end{tabular}

Customer comments:

Lab comments:

Comment objects:
Analytical Chemistry organization (Quality Services)

Page 1 of 1 P.0. Box 2009 oak Ridge, TN 378318244 (865) 574-2284

Lab Smp1 Id: A093150073 official Report Cust Smpl Id: 175 Mrad SRS

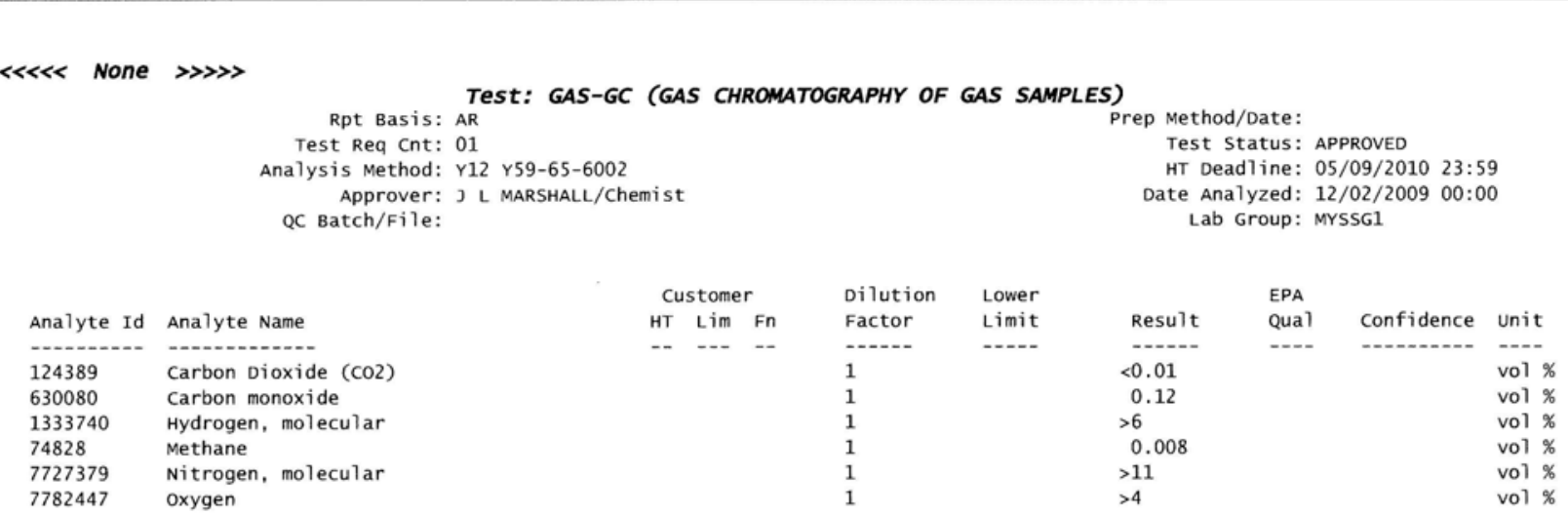

Comment objects:

Test: GAS-MS (Mass Spec. Analysis of Gas by VG 3001)

Rpt Basis: AR Test Req Cnt: 01

Analys is Method: ASO $\mathrm{Y} / \mathrm{P} 65-6011$

Approver: J L MARSHALL/Chemist

QC Batch/File:

Prep Method/Date:

Test Status: APPROVED

HT Deadline: 05/09/2010 23:59

Date Analyzed: 12/02/2009 00:00

Lab Group: MYSSG1

\begin{tabular}{lllllrl} 
& & \multicolumn{2}{c}{ Customer } & Dilution & Lower & EPA \\
Analyte Id & Analyte Name & HT Lim Fn & Factor & Limit & Result & Qual \\
Confidence Unit
\end{tabular}

Sample Test Comments: N20 estimate at 1.11\% [018053 12/09/2009 12:57:36]

Comment objects:

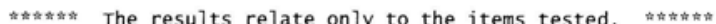

Results contained within this report are not Blank corrected unless specifically noted manth

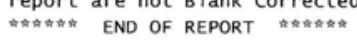

\section{UNCLASSIFIED}




\section{UNCLASSIFIED}

LIMS 03.08 .048

12/09/2009 13:10

Project: L MS MPO SCHUH GAS

Customer: L P BURNETT B 7 dg 9995 MS-8189 (865) 576-9141

\begin{tabular}{llcl} 
Matrix & $\begin{array}{l}\text { Chain of } \\
\text { Custody }\end{array}$ & $\begin{array}{l}\text { Work Agreement } \\
\text { Number }\end{array}$ & $\begin{array}{l}\text { Charge } \\
\text { Number }\end{array}$ \\
\hdashline GAS & 117882 & -81473 & -7450221 \\
\hdashline & Sample Description: \\
Location: ORNL \\
Sampler(s):
\end{tabular}

Analytical Chemistry organization (Quality Services)

P.o. Box 2009 oak Ridge, TN 378318244 (865) 574-2284

official Report
Page 1 of 1

Lab Smpl Id: A093150074

Cust Smpl Id: 175 Mrad 0.5M HNO3

Customer comments:

Lab comments:

Comment objects:

Test: GAS-GC (GAS CHROMATOGRAPHY OF GAS SAMPLES)

Rpt Basis: AR Test Req Cnt: 01

Analysis Method: Y12 Y59-65-6002

Approver: J L MARSHALL/Chemist

QC Batch/File:

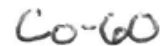

\begin{tabular}{|c|c|c|c|}
\hline $\begin{array}{l}\text { Date } \\
\text { Sampled }\end{array}$ & $\begin{array}{l}\text { Date } \\
\text { Received }\end{array}$ & $\begin{array}{l}\text { Date } \\
\text { Needed }\end{array}$ & $\begin{array}{l}\text { Date } \\
\text { Approved }\end{array}$ \\
\hline --- & ----- & ---- & $-\cdots--$ \\
\hline $11 / 10 / 200900: 00$ & $11 / 10 / 2009 \quad 15: 30$ & $12 / 09 / 2009 \quad 23: 59$ & $12 / 09 / 2009 \quad 13: 10$ \\
\hline
\end{tabular}

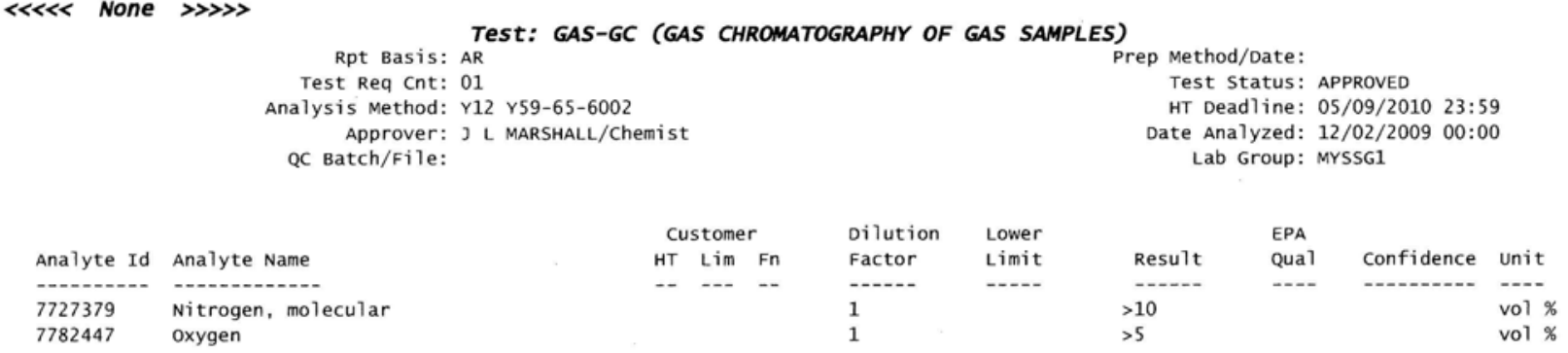

Sample Test Comments: Sample has air in it. [018053 12/09/2009 13:04:55]

Comment objects:

Test: GAS-MS (Mass Spec. Analysis of Gas by VG 3001)

Rpt Basis: AR Test Req Cnt: 01 Analysis Method: ASO Y/P65-6011

Approver: J L MARSHALL/Chemist QC Batch/File:
Prep Method/Date:

Test Status: APPROVED

HT Deadline: 05/09/2010 23:59

Date Analyzed: 12/02/2009 00:00 Lab Group: MYSSG1

\begin{tabular}{|c|c|c|c|c|c|c|c|c|c|c|}
\hline \multirow{3}{*}{ Analyte Id } & \multirow[b]{2}{*}{ Analyte Name } & \multicolumn{3}{|c|}{ Customer } & \multirow{2}{*}{$\begin{array}{l}\text { Dilution } \\
\text { Factor }\end{array}$} & \multirow{2}{*}{$\begin{array}{l}\text { Lower } \\
\text { Limit }\end{array}$} & \multirow[b]{2}{*}{ Result } & \multirow{2}{*}{$\begin{array}{l}\text { EPA } \\
\text { Qual }\end{array}$} & \multirow[b]{2}{*}{ Confidence } & \multirow[b]{2}{*}{ Unit } \\
\hline & & HT & Lim & $\mathrm{Fn}$ & & & & & & \\
\hline & - & -- & $\cdots$ & -- & $-\cdots-$ & --- & $\cdots-$ & --- & - n-m-n & --- \\
\hline 7727379 & Nitrogen, molecular & & & & 1 & & 78.5 & & & vol $\%$ \\
\hline 7782447 & oxygen & & & & 1 & & 20.4 & & & vol \% \\
\hline
\end{tabular}

Sample Test Comments: Sample has air in it. [018053 12/09/2009 13:04:08]

Comment objects:

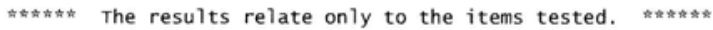

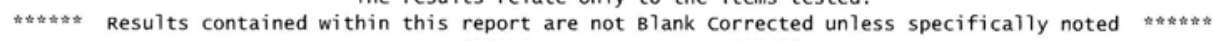

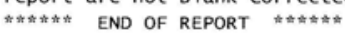

UNCLASSIFIED 


\section{UNCLASSIFIED}

LIMS 03.08 .048

02/17/2010 12:27

Project: L MS MPO SCHUH GAS

Customer: L P BURNETT Bldg 9995 MS-8189 (865) 576-9141

\begin{tabular}{|c|c|c|c|c|c|c|c|}
\hline Matrix & $\begin{array}{l}\text { Chain of } \\
\text { Custody }\end{array}$ & $\begin{array}{l}\text { Work Agreement } \\
\text { Number }\end{array}$ & $\begin{array}{l}\text { Charge } \\
\text { Number }\end{array}$ & $\begin{array}{l}\text { Date } \\
\text { Sampled }\end{array}$ & $\begin{array}{l}\text { Date } \\
\text { Received }\end{array}$ & $\begin{array}{l}\text { Date } \\
\text { Needed }\end{array}$ & $\begin{array}{l}\text { Date } \\
\text { Approved }\end{array}$ \\
\hline -ne- & 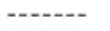 & ----- & ---- & $-\cdots-$ & ---- & $\cdots$ & --- \\
\hline GAS & 117931 & 81473 & $745 A 0221$ & $01 / 21 / 201000: 00$ & $01 / 21 / 2010 \quad 14: 30$ & $02 / 04 / 2010 \quad 23: 59$ & $02 / 17 / 2010 \quad 12: 27$ \\
\hline
\end{tabular}

Customer comments:

Lab comments:

Comment objects:

Proj Mgr: L P BURNETT (865) 576-9141

Sampler(s):
Analytical Chemistry organization (Quality Services)

Page 1 of 1

P.o. Box 2009 oak Ridge, TN 378318244 (865) 574-2284

Lab Smp1 Id: A100250139

official Report

Cust Smp 1 Id: HFIR 300Mrad SRS
Rpt Basis: AR Rpt Basis: AR
Test Req Cnt: 01

Analys is Method: Y12 Y59-65-6002

Approver: J L MARSHALL/Chemist

QC Batch/File:
Prep Method/Date:

Test Status: APPROVED

HT Deadline: 07/20/2010 23:59

Date Analyzed: 01/26/2010 00:00

Lab Group: MYSSG1

\begin{tabular}{|c|c|}
\hline Analyte Id & Analyte Name \\
\hline 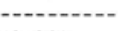 & - \\
\hline 124389 & Carbon Dioxide ( $\mathrm{CO} 2)$ \\
\hline 630080 & Carbon monoxide \\
\hline 1333740 & Hydrogen, molecular \\
\hline 74828 & Methane \\
\hline 7727379 & Nitrogen, molecular \\
\hline 7782447 & oxygen \\
\hline
\end{tabular}

\begin{tabular}{llll}
\multicolumn{2}{c}{ Customer } & Dilution & Lower \\
HT Lim Fn & Factor & Limit \\
- & & 1 & \\
& & 1 & \\
& & 1 & \\
& & 1 & \\
& & 1 & \\
& &
\end{tabular}

Result
-0.01
$<0.32$
$>30$
0.03
$>7$
$>25$

EPA

Qua? Confidence Unit

$<0.01$ vol $\%$

Comment objects: Test: GAS-MS (Mass Spec. Analysis of Gas by VG 3001)

\begin{tabular}{ll} 
& \\
Analyte Id & Analyte Name \\
\hline 7440371 & Argon \\
7440597 & Helium \\
1333740 & Hydrogen, molecular \\
74828 & Methane \\
7727379 & Nitrogen, molecular \\
7782447 & Oxygen
\end{tabular}

\begin{tabular}{llll}
\multicolumn{2}{c}{ Customer } & Dilution & Lower \\
HT Lim Fn & Factor & Limit \\
----- & -1 & \\
& & 1 & \\
& & 1 & \\
& & 1 & \\
& & & \\
& 1 &
\end{tabular}

$\begin{array}{llll} & \text { EPA } & & \\ \text { Result } & \text { Qual } & \text { Confidence } & \text { Unit } \\ -0.16 & & & \text { vol } \% \\ 20.11 & & & \text { vol } \% \\ 47.32 & & & \text { vol } \% \\ 0.02 & & & \text { vol } \% \\ 8.25 & & & \text { vol } \% \\ 22.89 & & & \text { vol } \%\end{array}$

Sample Test Comments: $\mathrm{N} 20=1.02 \%[018053 \quad 02 / 17 / 201010: 14: 35]$

Comment objects:

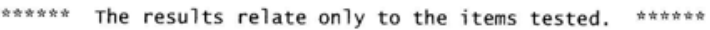

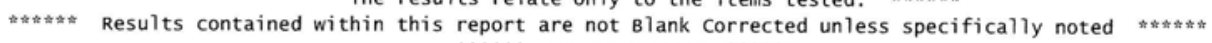

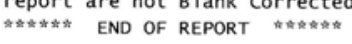

UNCLASSIFIED 


\section{UNCLASSIFIED}

LIMS 03.08 .048

$02 / 17 / 201012: 24$

project: L MS MPO SCHUH GAS

Customer: L P BURNETT B1dg 9995 MS-8189 (865) 576-9141

\begin{tabular}{|c|c|c|c|c|c|c|c|}
\hline Matrix & $\begin{array}{l}\text { Chain of } \\
\text { Custody }\end{array}$ & $\begin{array}{l}\text { Work Agreement } \\
\text { Number }\end{array}$ & $\begin{array}{l}\text { Charge } \\
\text { Number }\end{array}$ & $\begin{array}{l}\text { Date } \\
\text { Sampled }\end{array}$ & $\begin{array}{l}\text { Date } \\
\text { Received }\end{array}$ & $\begin{array}{l}\text { Date } \\
\text { Needed }\end{array}$ & $\begin{array}{l}\text { Date } \\
\text { Approved }\end{array}$ \\
\hline$-\ldots$ & --n-- & -....- & ----- & ----- & ----- & ---- & $-\ldots-n$ \\
\hline GAS & 117931 & 81473 & $745 A 0221$ & $01 / 21 / 201000: 00$ & $01 / 21 / 2010 \quad 14: 30$ & 02/04/2010 23:59 & $02 / 17 / 2010 \quad 12: 24$ \\
\hline
\end{tabular}

Customer comments:

Lab comments:

Comment objects: Proj Mgr: L. P BURNETT (865) 576-9141
Sample Status: APPROVED

Sampler(s):
Page 1 of 1 Lab Smp1 Id: A100250137 (865) $574-2284$ official Report

Cust Smp1 Id: HFIR 250Mrad SRS
Analytical Chemistry organization (Quality Services)

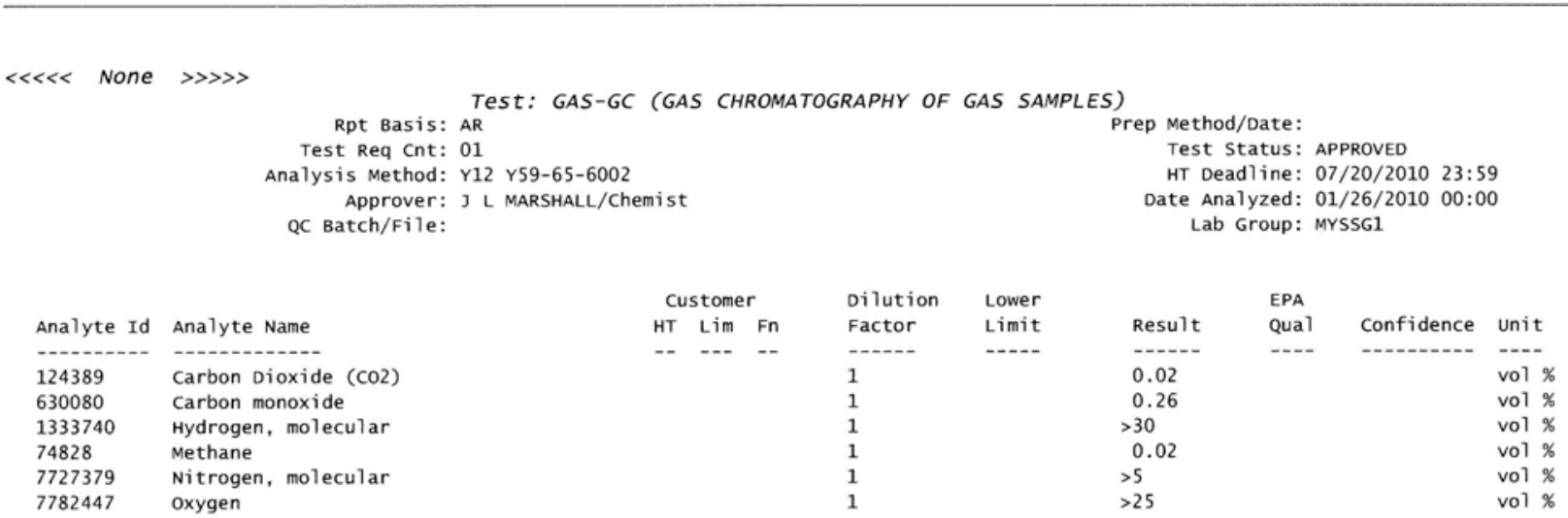

Comment objects: Test: GAS-MS (Mass Spec. Analysis of Gas by VG 3001)

Rpt Basis: AR Test Req Cnt: 01 Ana7ysis Method: ASO Y/P65-6011

Approver: J L MARSHALL/Chemist

QC Batch/File:

Prep Method/Date:

Test Status: APPROVED

HT Deadline: 07/20/2010 23:59 Date Analyzed: 01/26/2010 00:00 Lab Group: MYSSG1

\begin{tabular}{|c|c|c|c|c|c|c|c|c|c|c|}
\hline \multirow[b]{2}{*}{ Analyte Id } & \multirow[b]{2}{*}{ Analyte Name } & \multicolumn{3}{|c|}{ Customer } & \multirow{2}{*}{$\begin{array}{l}\text { Dilution } \\
\text { Factor }\end{array}$} & \multirow{2}{*}{$\begin{array}{l}\text { Lower } \\
\text { Limit }\end{array}$} & \multirow[b]{2}{*}{ Result } & \multirow{2}{*}{$\begin{array}{l}\text { EPA } \\
\text { Qual }\end{array}$} & \multirow[b]{2}{*}{ Confidence } & \multirow[b]{2}{*}{ Unit } \\
\hline & & HT & Lim & $\mathrm{Fn}$ & & & & & & \\
\hline - & - - & -- & $\cdots$ & -- & - n-- & $-\cdots$ & $\cdots-$ & --- & - n-n-n & $-\cdots$ \\
\hline 7440371 & Argon & & & & 1 & & 0.41 & & & vol \% \\
\hline 7440597 & Helium & & & & 1 & & 22.43 & & & vol $\%$ \\
\hline 1333740 & Hydrogen, molecular & & & & 1 & & 32.66 & & & vol $\%$ \\
\hline 74828 & Methane & & & & 1 & & 0.02 & & & vol $\%$ \\
\hline 7727379 & Nitrogen, molecular & & & & 1 & & 16.99 & & & vol $\%$ \\
\hline 7782447 & oxygen & & & & 1 & & 26.54 & & & vol \% \\
\hline
\end{tabular}

Sample Test Comments: $\mathrm{N} 20=.91 \%[018053 \quad 02 / 17 / 2010 \quad 10: 04: 52]$

Comment objects:

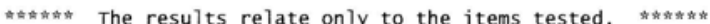

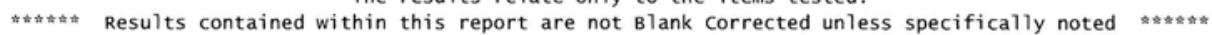

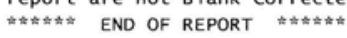

UNCLASSIFIED 


\section{UNCLASSIFIED}

LIMS 03.08 .048

12/09/2009 13:08

Project: L MS MPO SCHUH GAS

Customer: L P BURNETT Bldg 9995 MS-8189 (865) 576-9141

\begin{tabular}{llcl} 
Matrix & $\begin{array}{l}\text { Chain of } \\
\text { Custody }\end{array}$ & $\begin{array}{c}\text { Work Agreement } \\
\text { Number }\end{array}$ & $\begin{array}{l}\text { Charge } \\
\text { Number }\end{array}$ \\
\hdashline GAS & 117882 & -81473 & -745 A0221 \\
\hdashline & Sample Description: \\
Location: ORNL \\
Sampler(s):
\end{tabular}

Date

Sampled

$11 / 10 / 200900: 00$$$
\text { (1) }
$$

Date
Received

$11 / 10 / 200915: 30$
Page 1 of 1

Lab Smpl Id: A093150072

Customer comments:

Lab comments:

Comment objects:

Sample Test comments: Sample had air in it [018053 12/09/2009 12:56:25]

Comment objects:

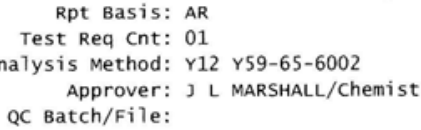

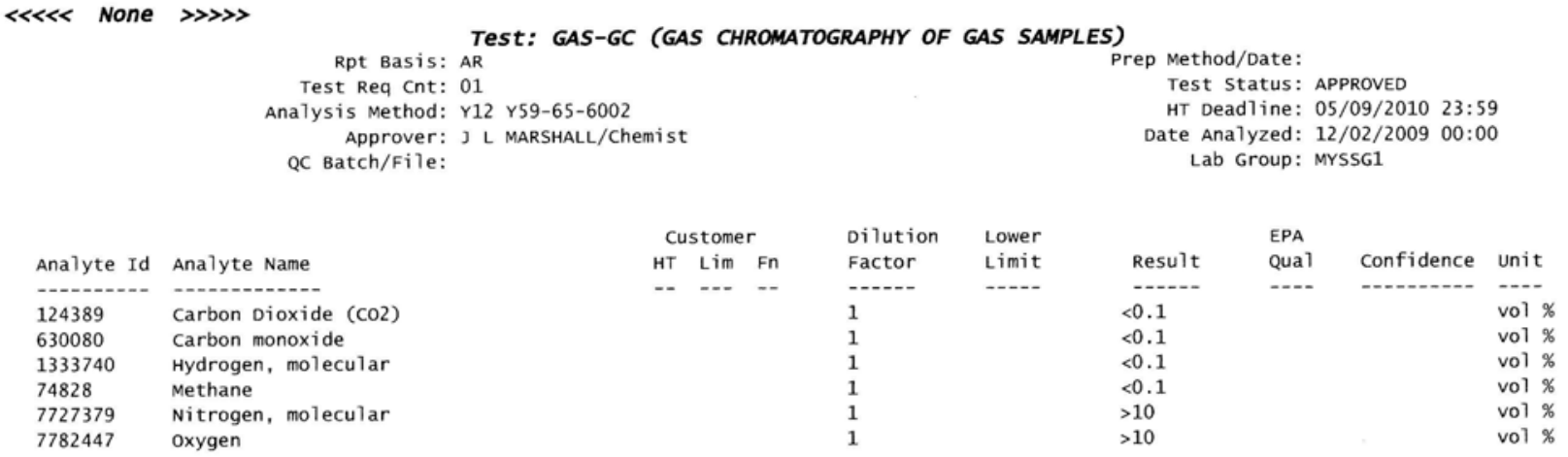

Test: GAS-MS (Mass Spec. Analysis of Gas by VG 3001)

Rpt Basis: AR Test Req Cnt: 01 Analysis Method: ASO Y/P65-6011 Approver: J L MARSHALL/Chemist QC Batch/File:
Prep Method/Date:

Test Status: APPROVED

HT Deadline: 05/09/2010 23:59

Date Analyzed: 12/02/2009 00:00

Lab Group: MYSSG1

\begin{tabular}{ll} 
Analyte Id & Analyte Name \\
\hline 7440371 & Argon \\
7440597 & Helium \\
1333740 & Hydrogen, molecular \\
74828 & Methane \\
7727379 & Nitrogen, molecular \\
7782447 & oxygen
\end{tabular}

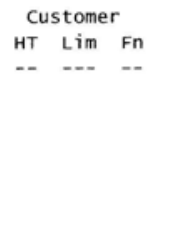

\begin{tabular}{ll} 
Dilution & Lower \\
Factor & Limit \\
\hdashline 1 & \\
1 & \\
1 & \\
1 & \\
1 & \\
1 &
\end{tabular}

Result
-0.48
$<0.01$
41.99
0.01
32.8
24.2

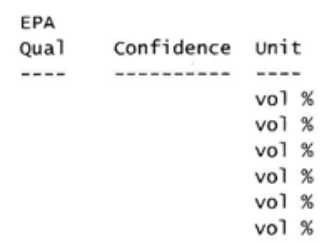

Sample Test Comments: N20 estimate .52\% [018053 12/09/2009 12:53:54]

Comment objects:

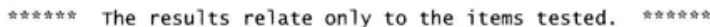

Results contained within this report are not Blank corrected unless specifically noted popkn

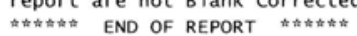

\section{UNCLASSIFIED}




\section{UNCLASSIFIED}

LIMS 03.08 .048

12/09/2009 13:09
Analytical Chemistry organization (Quality Services)

P.o. Box 2009 oak Ridge, TN 378318244 (865) 574-2284
Page 1 of 1

Lab Smp1 Id: A093150071

Project: L MS MPO SCHUH GAS

Proj Mgr: L P BURNETT (865) 576-9141

Customer: L P BURNETT B1dg 9995 MS-8189 (865) 576-9141

\begin{tabular}{|c|c|c|c|c|c|c|c|}
\hline Matrix & $\begin{array}{l}\text { Chain of } \\
\text { Custody }\end{array}$ & $\begin{array}{l}\text { Work Agreement } \\
\text { Number }\end{array}$ & $\begin{array}{l}\text { Charge } \\
\text { Number }\end{array}$ & $\begin{array}{l}\text { Date } \\
\text { Sampled }\end{array}$ & $\begin{array}{l}\text { Date } \\
\text { Received }\end{array}$ & $\begin{array}{l}\text { Date } \\
\text { Needed }\end{array}$ & $\begin{array}{l}\text { Date } \\
\text { Approved }\end{array}$ \\
\hline ---- & -n- & - & $\cdots-$ & -.-- & -.....- & -..-- & - n-n \\
\hline GAS & 117882 & 81473 & $745 A 0221$ & $11 / 10 / 200900: 00$ & $11 / 10 / 200915: 30$ & $12 / 09 / 2009 \quad 23: 59$ & $12 / 09 / 200913: 09$ \\
\hline
\end{tabular}

Customer comments:

Lab comments:

Comment objects:

Test: GAS-GC (GAS CHROMATOGRAPHY OF GAS SAMPLES)

Rpt Basis: AR Test Req Cnt: 01

Analysis Method: Y12 Y59-65-6002

Approver: J L MARSHALL/Chemist

QC Batch/File:
Prep Method/Date:

Test Status: APPROVED

HT Deadline: 05/09/2010 23:59

Date Analyzed: 12/02/2009 00:00

Lab Group: MYSSG1

\begin{tabular}{|c|c|c|c|c|c|c|c|c|c|c|}
\hline \multirow{3}{*}{ Analyte Id } & \multirow[b]{2}{*}{ Analyte Name } & \multicolumn{3}{|c|}{ Customer } & \multirow{2}{*}{$\begin{array}{l}\text { Dilution } \\
\text { Factor }\end{array}$} & \multirow{2}{*}{$\begin{array}{l}\text { Lower } \\
\text { Limit }\end{array}$} & \multirow[b]{2}{*}{ Result } & \multirow{2}{*}{$\begin{array}{l}\text { EPA } \\
\text { Qual }\end{array}$} & \multirow[b]{2}{*}{ Confidence } & \multirow[b]{2}{*}{ Unit } \\
\hline & & HT & Lim & $\mathrm{Fn}$ & & & & & & \\
\hline & - & -- & $\cdots$ & -- & ---- & $-\cdots$ & ----- & --- & - & $\cdots$ \\
\hline 124389 & Carbon Dioxide ( $\mathrm{CO} 2)$ & & & & 1 & & $<0.01$ & & & vol \% \\
\hline 630080 & Carbon monoxide & & & & 1 & & $<0.01$ & & & vol \% \\
\hline 1333740 & Hydrogen, molecular & & & & 1 & & $>10$ & & & vol \% \\
\hline 74828 & Methane & & & & 1 & & 0.01 & & & vol $\%$ \\
\hline 7727379 & Nitrogen, molecular & & & & 1 & & $>7$ & & & vol $\%$ \\
\hline 7782447 & oxygen & & & & 1 & & $>3$ & & & vol \% \\
\hline
\end{tabular}

Comment objects:

\section{Test: GAS-MS (Mass Spec. Analysis of Gas by VG 3001)}

Rpt Basis: AR Test Req Cnt: 01

Analysis Method: ASO Y/P65-6011

Approver: J L MARSHALL/Chemist

QC Batch/File:

Prep Method/Date:

Test Status: APPROVED

HT Deadline: 05/09/2010 23:59

Date Analyzed: 12/02/2009 00:00

Lab Group: MYSSG1

\begin{tabular}{ll} 
Analyte Id & Analyte Name \\
\hline 7440371 & Argon \\
7440597 & Helium \\
1333740 & Hydrogen, molecular \\
74828 & Methane \\
7727379 & Nitrogen, molecular \\
7782447 & oxygen
\end{tabular}

\begin{tabular}{llll}
\multicolumn{2}{c}{ Customer } & Dilution & Lower \\
HT Lim Fn & Factor & Limit \\
$--\quad--$ & -- & ---- & \\
& & 1 & \\
& & 1 & \\
& & 1 & \\
& & 1 & \\
& & 1 & \\
& & &
\end{tabular}

Result
-0.65
0.01
34.8
0.008
29.9
34.15

EPA

Qual Confidence unit

---- -----1---- ---

vol $\%$

vol \%

vol $\%$

vol $\%$

vol \%

vol \%

Sample Test Comments: N20 estimate at .49\% [018053 12/09/2009 12:46:57]

Comment objects:

the results relate only to the items tested.

Results contained within this report are not Blank corrected unless specifically noted

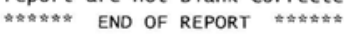

UNCLASSIFIED 


\section{UNCLASSIFIED}

LIMS 03.08 .048

03/08/2010 13:36

Project: L MS MPO SCHUH GAS

Customer: L P BURNETT B 7 dg 9995 MS-8189 (865) 576-9141

\begin{tabular}{|c|c|c|c|c|c|c|c|}
\hline Matrix & $\begin{array}{l}\text { Chain of } \\
\text { Custody }\end{array}$ & $\begin{array}{l}\text { Work Agreement } \\
\text { Number }\end{array}$ & $\begin{array}{l}\text { Charge } \\
\text { Number }\end{array}$ & $\begin{array}{l}\text { Date } \\
\text { Sampled }\end{array}$ & $\begin{array}{l}\text { Date } \\
\text { Received }\end{array}$ & $\begin{array}{l}\text { Date } \\
\text { Needed }\end{array}$ & $\begin{array}{l}\text { Date } \\
\text { Approved }\end{array}$ \\
\hline---- & ----- & --n- & $-\cdots$ & - n- & - n- & -n- & - n-n- \\
\hline GAS & 117935 & 81473 & $745 A 0221$ & $02 / 23 / 2010 \quad 00: 00$ & $02 / 23 / 2010 \quad 14: 30$ & $03 / 09 / 2010 \quad 23: 59$ & $03 / 08 / 201013: 36$ \\
\hline
\end{tabular}

Customer comments:

Lab comments:

Comment objects:

Proj Mgr: L P BURNETT (865) 576-9141
Analytical Chemistry organization (Quality Services)

Page 1 of 1 P.o. Box 2009 oak Ridge, TN 378318244 (865) 574-2284

Lab Smpl Id: A100550036 official Report

$\ll<<$ None $\gg \gg>>$

Rpt Basis: AR Test: GAS-GC (GAS CHROMATOGRAPHY OF GAS SAMPLES) Test Req Cnt: 01 Analysis Method: Y12 Y59-65-6002

Approver: J L MARSHALL/Chemist

QC Batch/File:

Prep Method/Date:

Test Status: APPROVED

HT Deadline: 08/22/2010 23:59 Date Analyzed: 03/04/2010 00:00 Lab Group: MYSSG1

\begin{tabular}{|c|c|c|c|c|c|c|c|c|c|c|}
\hline \multirow[b]{2}{*}{ Analyte Id } & \multirow[b]{2}{*}{ Analyte Name } & \multicolumn{3}{|c|}{ Customer } & \multirow{2}{*}{$\begin{array}{l}\text { Dilution } \\
\text { Factor }\end{array}$} & \multirow{2}{*}{$\begin{array}{l}\text { Lower } \\
\text { Limit }\end{array}$} & \multirow[b]{2}{*}{ Result } & \multirow{2}{*}{$\begin{array}{l}\text { EPA } \\
\text { Qual }\end{array}$} & \multirow[b]{2}{*}{ Confidence } & \multirow[b]{2}{*}{ Unit } \\
\hline & & HT & Lim & $\mathrm{Fn}$ & & & & & & \\
\hline 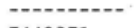 & $-2-20-0-1$ & -- & --- & -- & $---\cdot-$ & $-\cdots$ & ----- & $--\cdot$ & - & --- \\
\hline 7440371 & Argon & & & & 1 & & 0.34 & & & vol \% \\
\hline 124389 & Carbon Dioxide $(\mathrm{CO} 2)$ & & & & 1 & & $<0.01$ & & & vol \% \\
\hline 630080 & Carbon monoxide & & & & 1 & & 0.05 & & & vol \% \\
\hline 1333740 & Hydrogen, molecular & & & & 1 & & $>5$ & & & vol \% \\
\hline 74828 & Methane & & & & 1 & & 0.01 & & & vol \% \\
\hline 7727379 & Nitrogen, molecular & & & & 1 & & $>5$ & & & vol \% \\
\hline
\end{tabular}

Comment objects: Test: GAS-MS (Mass Spec. Analysis of Gas by VG 3001)

Rpt Basis: AR Test Req Cnt: 01 Analysis Method: ASO Y/P65-6011 Approver: J L MARSHALL/Chemist QC Batch/File:

Prep Method/Date:

Test Status: APPROVED

HT Deadline: $08 / 22 / 2010 \quad 23: 59$ Date Analyzed: 03/04/2010 00:00 Lab Group: MYSSG1

\begin{tabular}{ll} 
Analyte Id & Analyte Name \\
\hline 7440371 & Argon \\
7440597 & Helium \\
1333740 & Hydrogen, molecular \\
74828 & Methane \\
7727379 & $\begin{array}{l}\text { Nitrogen, molecular } \\
7782447\end{array}$ \\
& oxygen
\end{tabular}

Customer Dilution Lower

HT Lim Fn Factor Limit

$----$

\section{1}

----

$\begin{array}{llll}\text { Result } & \text { Qual } & \text { Confidence } & \text { Unit } \\ -0.45 & & & - \text { vol } \% \\ 0.1 & & & \text { vol } \% \\ 57.26 & & & \text { vol } \% \\ 0.01 & & \text { vol } \% \\ 38.94 & & \text { vol } \% \\ 0.01 & & \text { vol } \%\end{array}$

Sample Test Comments: $\mathrm{N} 2 \mathrm{O}+\mathrm{CO} 2=3.07 \%[018053 \quad 03 / 08 / 2010 \quad 13: 26: 10]$

Comment objects:

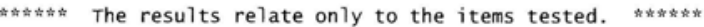

Results contained within this report are not Blank corrected unless specifically noted $m \neq n$

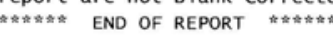

\section{UNCLASSIFIED}




\section{UNCLASSIFIED}

LIMS 03.08.048

03/08/2010 13:35

Project: L MS MPO SCHUH GAS

Customer: L P BURNETT B1dg 9995 MS-8189 (865) 576-9141

\begin{tabular}{|c|c|c|c|c|c|c|c|}
\hline Matrix & $\begin{array}{l}\text { Chain of } \\
\text { Custody }\end{array}$ & $\begin{array}{l}\text { Work Agreement } \\
\text { Number }\end{array}$ & $\begin{array}{l}\text { Charge } \\
\text { Number }\end{array}$ & $\begin{array}{l}\text { Date } \\
\text { Sampled }\end{array}$ & $\begin{array}{l}\text { Date } \\
\text { Received }\end{array}$ & $\begin{array}{l}\text { Date } \\
\text { Needed }\end{array}$ & $\begin{array}{l}\text { Date } \\
\text { Approved }\end{array}$ \\
\hline -n- & $-----\overline{-}$ & - n- & $-\cdots-$ & - - & --.--- & $-\cdots-$ & - \\
\hline GAS & 117935 & 81473 & $745 A 0221$ & $02 / 23 / 2010 \quad 00: 00$ & $02 / 23 / 2010 \quad 14: 30$ & $03 / 09 / 2010 \quad 23: 59$ & $03 / 08 / 2010 \quad 13: 35$ \\
\hline
\end{tabular}

Customer comments:

Lab Comments:

Comment objects:
Page 1 of 1 Lab Smp 1 Id: A100550034
Cust Smp1 Id: HFIR Hanford 250 Mrad
Proj Mgr: L P BURNETT (865) 576-9141
Analytical Chemistry organization (Quality Services)
official Report

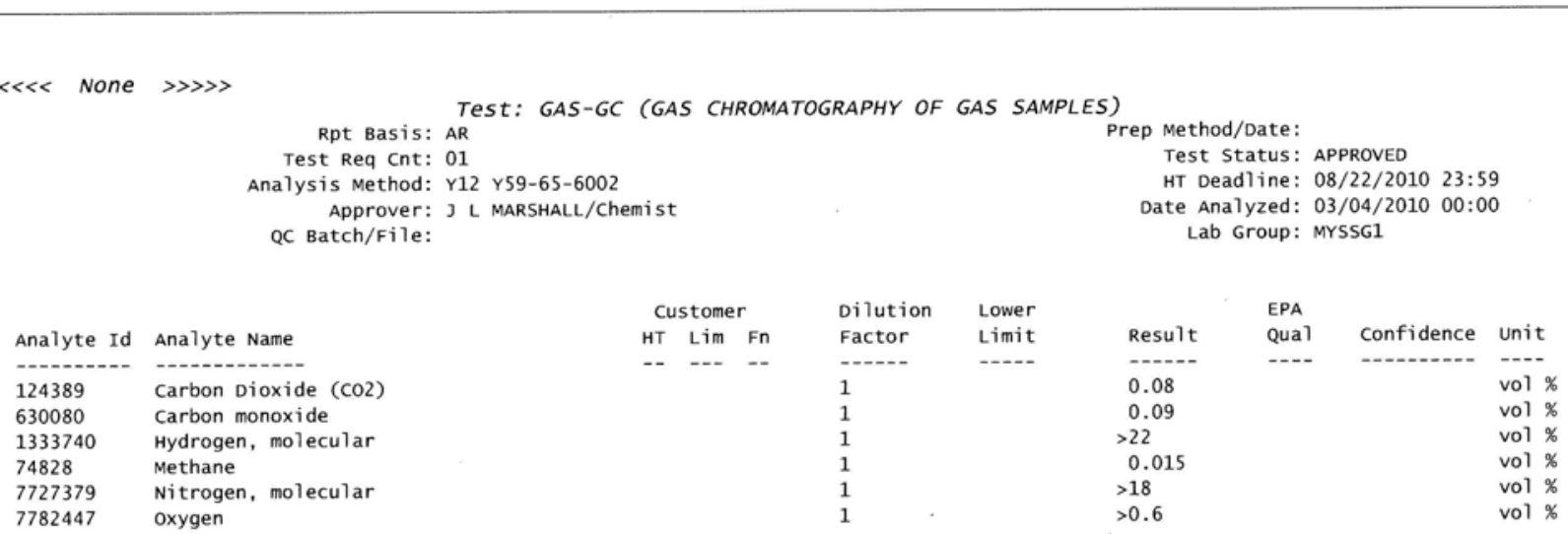

Comment objects:

Test: GAS-MS (Mass Spec. Analysis of Gas by VG 3001)

Rpt Basis: AR Test Req Cnt: 01 Analysis Method: ASO Y/P65-6011

Approver: J L MARSHALL/Chemist

QC Batch/File:
Prep Method/Date:

Test Status: APPROVED

HT Deadline: $08 / 22 / 201023: 59$ Date Analyzed: 03/04/2010 00:00

Lab Group: MYSSGI

\begin{tabular}{|c|c|c|c|c|c|c|c|c|c|c|}
\hline \multirow{3}{*}{ Analyte Id } & \multirow[b]{2}{*}{ Analyte Name } & \multicolumn{3}{|c|}{ Customer } & \multirow{2}{*}{$\begin{array}{l}\text { Dilution } \\
\text { Factor }\end{array}$} & \multirow{2}{*}{$\begin{array}{l}\text { Lower } \\
\text { Limit }\end{array}$} & \multirow[b]{2}{*}{ Result } & \multirow{2}{*}{$\begin{array}{l}\text { EPA } \\
\text { Qua } 7\end{array}$} & \multirow[b]{2}{*}{ Confidence } & \multirow[b]{2}{*}{ Unit } \\
\hline & & HT & Lim & $\mathrm{Fn}$ & & & & & & \\
\hline & - & -- & -- & -- & -...- & --- & $\cdots-$ & $\cdots$ & - n-m- & $\cdots$ \\
\hline 7440371 & Argon & & & & 1 & & 0.31 & & & vol $*$ \\
\hline 7440597 & Helium & & & & 1 & & $<0.1$ & & & vol $x$ \\
\hline 1333740 & Hydrogen, molecular & & & & 1 & & 67.55 & & & vol $\%$ \\
\hline 74828 & Methane & & & & 1 & & 0.02 & & & vol $x$ \\
\hline 7727379 & Nitrogen, molecular & & & & 1 & & 26.91 & & & vol $\%$ \\
\hline 7782447 & oxygen & & & & 1 & & 0.76 & & & vol $\%$ \\
\hline
\end{tabular}

Sample Test Comments: $\mathrm{N} 2 \mathrm{O}+\mathrm{CO} 2=4.0 \%[018053 \quad 03 / 08 / 2010 \quad 13: 24: 22]$

Comment objects:

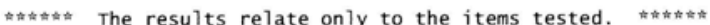

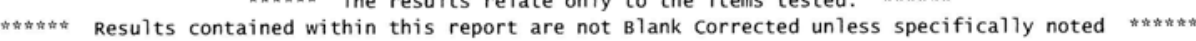

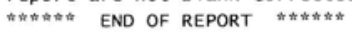

\section{UNCLASSIFIED}




\section{UNCLASSIFIED}

LIMS 03.08 .048

03/24/2010 10:59

Project: L MS MPO SCHUH GAS

Customer: L P BURNETT B1dg 9995 MS-8189 (865) 576-9141

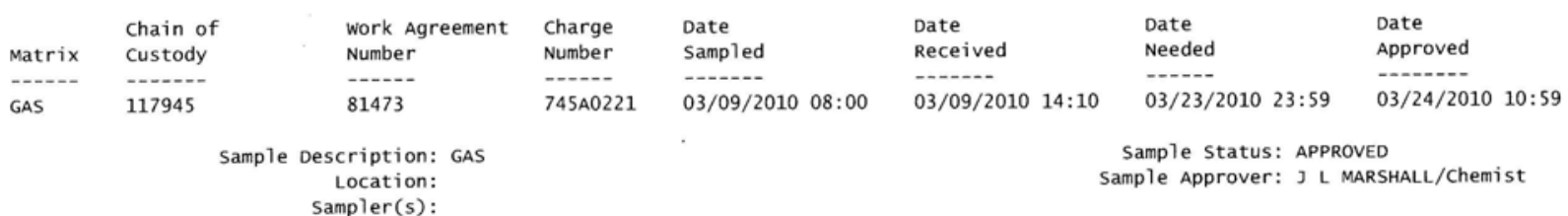

Customer comments:

Lab Comments:

Comment objects:

Proj Mgr: L P BURNETT (865) 576-9141

Cust Smpl Id: HFIR HANFORD 200
Analytical Chemistry organization (Quality Services)

Page 1 of 1

P.o. Box 2009 oak Ridge, TN 378318244 (865) 574-2284

Lab Smp1 Id: A100690175 


\section{UNCLASSIFIED}

LIMS 03.08 .048

12/09/2009 12:47

Project: L MS MPO SCHUH GAS

Customer: L P BURNETT B1dg 9995 MS-8189 (865) 576-9141

\begin{tabular}{llcl} 
Matrix & $\begin{array}{l}\text { Chain of } \\
\text { Custody }\end{array}$ & $\begin{array}{c}\text { Work Agreement } \\
\text { Number }\end{array}$ & $\begin{array}{l}\text { Charge } \\
\text { Number }\end{array}$ \\
\hdashline GAS & 117882 & -81473 & 745 A0221 \\
& Sample Description: Hanford Simulant \\
Location: ORNL \\
Sampler(s):
\end{tabular}

Customer comments:

Lab comments:

Comment objects:
Analytical Chemistry Organization (Quality Services) P.o. Box 2009 oak Ridge, TN 378318244 (865) 574-2284

Page 1 of 1 official Report

Proj Mgr: L P BURNETT (865) 576-9141

Lab Smp1 Id: A093150070

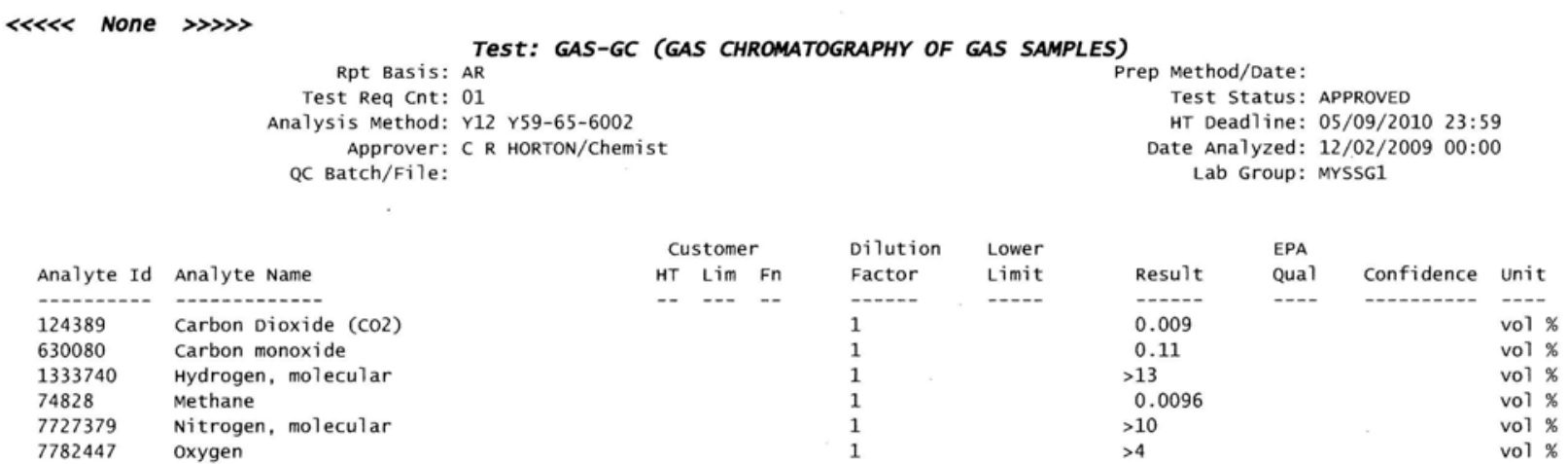

Comment objects:

Test: GAS-MS (Mass Spec. Analysis of Gas by VG 3001)

Rpt Basis: AR Test Req Cnt: 01 Analysis Method: ASO $\mathrm{Y} / \mathrm{P} 65-6011$

Approver: C R HORTON/Chemist QC Batch/File:

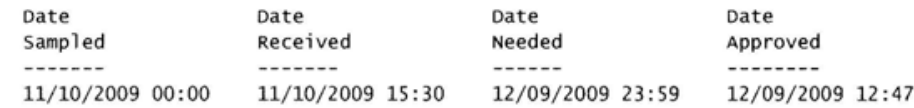

Sample status: APPROVED

Sample Approver: C R HORTON/Chemist

Prep Method/Date:

Test Status: APPROVED

HT Deadline: 05/09/2010 23:59

Date Analyzed: 12/02/2009 00:00

Lab Group: MYSSG1

\begin{tabular}{ll} 
Analyte Id & Analyte Name \\
\hline 7440371 & Argon \\
7440597 & Helium \\
1333740 & Hydrogen, molecular \\
74828 & Methane \\
7727379 & Nitrogen, molecular \\
7782447 & oxygen
\end{tabular}

\begin{tabular}{|c|c|c|c|c|c|c|c|c|}
\hline & istome & & Dilution & Lower & & EPA & & \\
\hline HT & Lim & $\mathrm{Fn}$ & Factor & Limit & Result & Qual & Confidence & Unit \\
\hline-- & $--\cdot$ & -- & --- & -...- & ---- & -..- & - & --- \\
\hline & & & 1 & & 0.44 & & & vol $x$ \\
\hline & & & 1 & & $<0.01$ & & & vol $x$ \\
\hline & & & 1 & & 44.01 & & & vol $\%$ \\
\hline & & & 1 & & 0.008 & & & vol $\%$ \\
\hline & & & 1 & & 34.8 & & & vol $\%$ \\
\hline & & & 1 & & 19.77 & & & vol $\%$ \\
\hline
\end{tabular}

Sample Test Comments: N20 estimate at .94\% [018053 12/09/2009 12:42:37]

Comment objects:

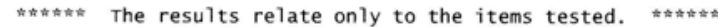

\#tw

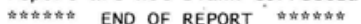

UNCLASSIFIED 
UNCLASSIFIED

LIMS 03.08 .048

03/24/2010 10:59

Project: L MS MPO SCHUH GAS

Customer: L P BURNETT Bldg 9995 MS-8189 (865) 576-9141

\begin{tabular}{|c|c|c|c|c|c|c|c|}
\hline Matrix & $\begin{array}{l}\text { Chain of } \\
\text { Custody }\end{array}$ & $\begin{array}{l}\text { Work Agreement } \\
\text { Number }\end{array}$ & $\begin{array}{l}\text { Charge } \\
\text { Number }\end{array}$ & $\begin{array}{l}\text { Date } \\
\text { Sampled }\end{array}$ & $\begin{array}{l}\text { Date } \\
\text { Received }\end{array}$ & $\begin{array}{l}\text { Date } \\
\text { Needed }\end{array}$ & $\begin{array}{l}\text { Date } \\
\text { Approved }\end{array}$ \\
\hline$-\cdots-$ & - n- & - & ------ & -...... & $-\ldots-$ & - - & - \\
\hline GAS & 117945 & 81473 & $745 A 0221$ & 03/09/2010 08:00 & $03 / 09 / 2010 \quad 14: 10$ & $03 / 23 / 2010 \quad 23: 59$ & $03 / 24 / 2010 \quad 10: 59$ \\
\hline
\end{tabular}

Customer comments:

Lab comments:

Comment objects:

$\ll<<$ None $\gg \gg>>$

Rpt Basis: AR Test: GAS-GC (GAS CHROMATOGRAPHY OF GAS SAMPLES)

Rpt Basis: AR
Test Req Cnt: 01

Analysis Method: Y12 Y59-65-6002

Approver: J L MARSHALL/Chemist

QC Batch/File:

Analytical Chemistry organization (Quality Services)

Page 1 of 1

P.0. Box 2009 oak Ridge, TN 378318244 (865) 574-2284

Lab Smp1 Id: A100690174

official Report Cust Smpl Id: HFIR H2O 300

Proj Mgr: L P BURNETT (865) 576-9141

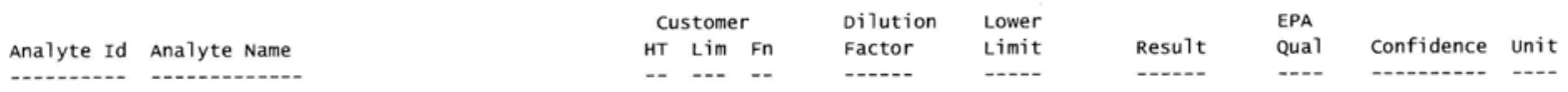

Sample Test Comments: No gas in this section of tube. [018053 03/24/2010 10:47:01]

Comment objects:

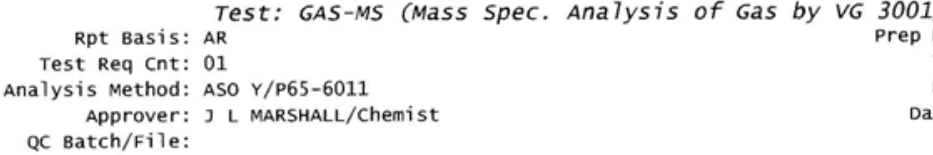

\begin{tabular}{|c|c|c|c|c|c|c|c|c|}
\hline \multicolumn{3}{|c|}{ Customer } & \multirow{2}{*}{$\begin{array}{l}\text { Dilution } \\
\text { Factor }\end{array}$} & \multirow{2}{*}{$\begin{array}{l}\text { Lower } \\
\text { Limit }\end{array}$} & & \multirow{2}{*}{$\begin{array}{l}\text { EPA } \\
\text { Qual }\end{array}$} & \multirow[b]{2}{*}{ Confidence } & \multirow[b]{2}{*}{ Unit } \\
\hline HT & Lim & $\mathrm{Fn}$ & & & Result & & & \\
\hline-- & -- & -- & - - & $\ldots$ & - - & --- & 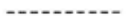 & $\cdots$ \\
\hline & & & 1 & & 0.37 & & & vol \% \\
\hline & & & 1 & & $<0.1$ & & & vol \% \\
\hline & & & 1 & & 80.8 & & & vol \% \\
\hline & & & 1 & & 0.04 & & & vol \% \\
\hline & & & 1 & & 17.48 & & & vol \% \\
\hline & & & 1 & & 0.6 & & & vol $\%$ \\
\hline
\end{tabular}

\begin{tabular}{ll} 
Analyte Id & Analyte Name \\
\hline 7440371 & Argon \\
7440597 & Helium \\
1333740 & Hydrogen, molecular \\
74828 & Methane \\
7727379 & Nitrogen, molecular \\
7782447 & Oxygen
\end{tabular}

Sample Test Comments: $N 20=.04 \%[018053 \quad 03 / 24 / 2010 \quad 10: 48: 22]$

Comment objects:

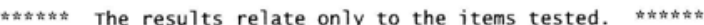

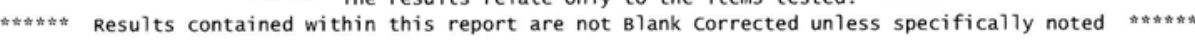

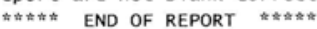

UNCLASSIFIED 


\section{UNCLASSIFIED}

LIMS 03.08.048

$02 / 17 / 201012: 29$

Project: L MS MPO SCHUH GAS

Customer: L P BURNETT B1dg 9995 MS-8189 (865) 576-9141

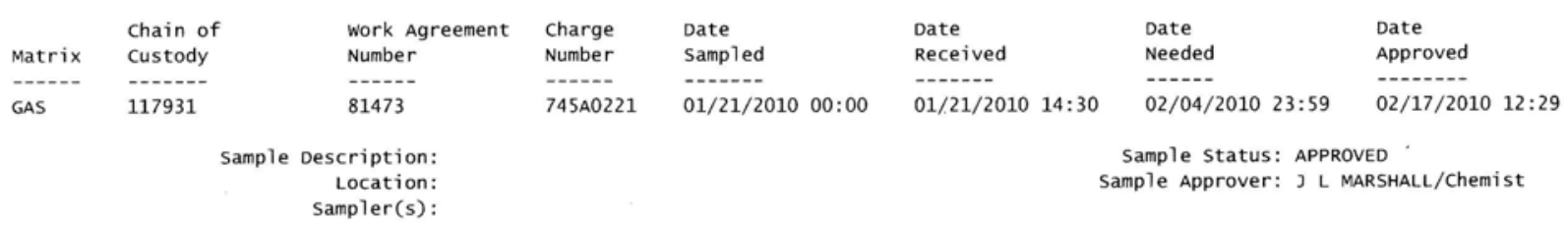

Customer comments:

Lab comments:

Comment objects:

Proj Mgr: L P BURNETT (865) 576-9141

Sampler(s):

Sample Status: APPROVED
Analytical Chemistry organization (Quality Services)

Page 1 of 1

P.o. Box 2009 oak Ridge, TN 378318244 (865) 574-2284

Lab Smp1 Id: A100250141

official Report Cust Smpl Id: HFIR water 250Mrad

$\ll<<$ None $\gg \gg>$

Rpt Basis: AR Test Req Cnt: 01

Analysis Method: Y12 Y59-65-6002

Approver: J L MARSHALL/Chemist

QC Batch/File: Test: GAS-GC (GAS CHROMATOGRAPHY OF GAS SAMPLES)

Prep Method/Date:

Test Status: APPROVED

HT Deadline: 07/20/2010 23:59

Date Analyzed: 02/16/2010 00:00

Lab Group: MYSSG1

$\begin{array}{lllllrl} & & \text { Customer } & \text { Dilution } & \text { Lower } & \text { EPA } \\ \text { Analyte Id } & \text { Analyte Name } & \text { HT Lim Fn } & \text { Factor } & \text { Limit } & \text { Result } & \text { Qual } \\ \text { Confidence Unit }\end{array}$

Sample Test Comments: $\mathrm{C} 2 \mathrm{H} 6=.01 \%[018053 \quad 02 / 17 / 201012: 21: 59]$

Comment objects:

Rpt Basis: $A R$

Test: GAS-MS (Mass Spec. Analysis of Gas by VG 3001) Test Req Cnt: 01

Analysis Method: ASO Y/P65-6011

Approver: J L MARSHALL/Chemist

QC Batch/File:
Prep Method/Date:

Test Status: APPROVED

HT Deadline: 07/20/2010 23:59 Date Analyzed: 02/16/2010 00:00 Lab Group: MYSSG1

\begin{tabular}{ll} 
Analyte Id & Analyte Name \\
\hline 7440371 & Argon \\
7440597 & Helium \\
1333740 & Hydrogen, molecular \\
74828 & Methane \\
7727379 & $\begin{array}{l}\text { Nitrogen, molecular } \\
\text { oxygen }\end{array}$
\end{tabular}

Customer Dilution Lower HT Lim Fn Factor Limit Limit

$\begin{array}{llll}\text { Eesu1t } & \text { QPA } & & \\ -0.22 & & & \text { Unit } \\ 32.48 & & & - \text { vol } \% \\ 59.19 & & & \text { vol } \% \\ 0.06 & & & \text { vol } \% \\ 7.45 & & \text { vol } \% \\ 0.07 & & & \text { vol } \% \\ & & & \text { vol } \%\end{array}$

Sample Test Comments: $\mathrm{N} 20=.11 \%[018053 \quad 02 / 17 / 201012: 20: 02]$

Comment objects:

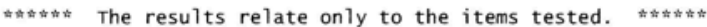

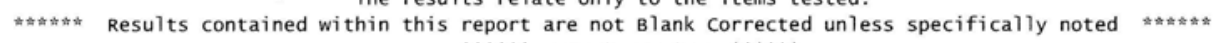

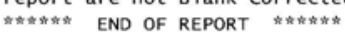

UNCLASSIFIED 


\section{UNCLASSIFIED}

Analytical Chemistry organization (Quality Services)

Page 1 of 1

LIMS 03.08 .048

12/09/2009 12:48

Customer: L P BURNETT Bldg 9995 MS-8189 (865) 576-9141

\begin{tabular}{|c|c|c|c|c|c|c|c|c|}
\hline Matrix & $\begin{array}{l}\text { Chain of } \\
\text { Custody }\end{array}$ & $\begin{array}{l}\text { Work Agreement } \\
\text { Number }\end{array}$ & $\begin{array}{l}\text { Charge } \\
\text { Number }\end{array}$ & $\begin{array}{l}\text { Date } \\
\text { Sampled }\end{array}$ & $\begin{array}{l}\text { Date } \\
\text { Received }\end{array}$ & . & $\begin{array}{l}\text { Date } \\
\text { Needed }\end{array}$ & $\begin{array}{l}\text { Date } \\
\text { Approved }\end{array}$ \\
\hline ----- & - n- & - & $\cdots-$ & - - - & -.....- & & $\cdots-$ & $-\cdots-1$ \\
\hline GAS & 117882 & 81473 & 745A0221 & $11 / 10 / 200900: 00$ & $11 / 10 / 2009$ & $15: 30$ & $12 / 09 / 2009 \quad 23: 59$ & $12 / 09 / 2009 \quad 12: 48$ \\
\hline
\end{tabular}

Customer comments:

Lab Comments:

Comment objects:
Lab Smpl Id: A093150069

$$
\text { official Report Cust Smpl Id: Water } 200 \mathrm{Mrad}
$$

mple status: APPROVED

Sampler(s):

$\longleftrightarrow<$ None $\gg \gg>$

Test: GAS-GC (GAS CHROMATOGRAPHY OF GAS SAMPLES)

Rpt Basis: AR Test Req Cnt: 01

Analysis Method: Y12 Y59-65-6002

Approver: C R HORTON/Chemist

QC Batch/File:

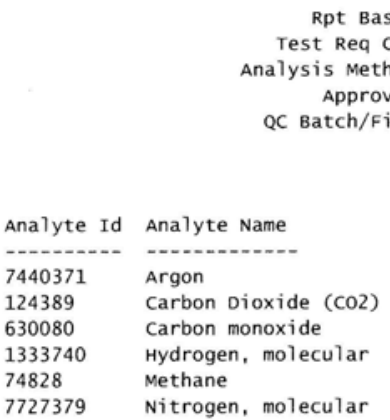

Prep Method/Date:

Test Status: APPROVED

HT Deadline: 05/09/2010 23:59

Date Analyzed: 12/02/2009 00:00

Lab Group: MYSSG1

Comment objects:

\begin{tabular}{|c|c|c|c|c|c|c|c|c|}
\hline \multicolumn{3}{|c|}{ Customer } & \multirow{2}{*}{$\begin{array}{l}\text { Dilution } \\
\text { Factor }\end{array}$} & \multirow{2}{*}{$\begin{array}{l}\text { Lower } \\
\text { Limit }\end{array}$} & & \multicolumn{2}{|l|}{ EPA } & \multirow[b]{2}{*}{ Unit } \\
\hline HT & Lim & $\mathrm{Fn}$ & & & Result & Qua] & Confidence & \\
\hline-- & -- & -- & -..-.- & -...- & -..-. & --- & - - & --.- \\
\hline & & & 1 & & 0.4 & & & vol \% \\
\hline & & & 1 & & 0.002 & & & vol \% \\
\hline & & & 1 & & 0.004 & & & vol \% \\
\hline & & & 1 & & $>5$ & & & vol \% \\
\hline & & & 1 & & 0.09 & & & vol \% \\
\hline & & & 1 & & $>5$ & & & vol \% \\
\hline
\end{tabular}

Test: GAS-MS (Mass Spec. Analysis of Gas by VG 3001)

Rpt Basis: AR Test Req Cnt: 01

Analysis Method: ASO $\mathrm{Y} / \mathrm{P} 65-6011$

QC Batch/File:

Test Status: APPROVED

Date Analyzed: 12/02/2009 00:00 Lab Group: MYSSG1

\begin{tabular}{ll} 
Analyte Id & Analyte Name \\
\hdashline 7440371 & Argon \\
124389 & Carbon Dioxide (CO2) \\
7440597 & Helium \\
1333740 & Hydrogen, molecular \\
74828 & Methane \\
7727379 & Nitrogen, molecular \\
7782447 & Oxygen
\end{tabular}

Customer Dilution Lower HT Lim Fn Factor Limit

\begin{tabular}{|c|c|c|c|}
\hline & $\begin{array}{l}\text { EPA } \\
\text { Qual }\end{array}$ & Confidence & Unit \\
\hline$-\cdots$ & $-\ldots$ & - & $\ldots$ \\
\hline 0.34 & & & vol $\%$ \\
\hline 0.01 & & & vol $\%$ \\
\hline 0.01 & & & vol $\%$ \\
\hline 72.04 & & & vol \% \\
\hline 0.09 & & & vol \% \\
\hline 27.47 & & & vol \% \\
\hline
\end{tabular}

Sample Test Comments: $\operatorname{NOx}(\mathrm{N} 2 \mathrm{O})=<.01 \%[018053 \quad 12 / 09 / 2009 \quad 12: 38: 30]$

Comment objects:

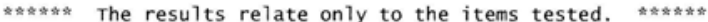

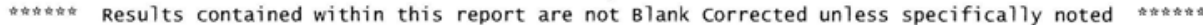

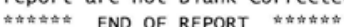

UNCLASSIFIED 


\section{UNCLASSIFIED}

LIMS 03.08 .048

02/17/2010 12:30

Project: L MS MPO SCHUH GAS

Customer: L P BURNETT B Bldg 9995 MS-8189 (865) 576-9141

\begin{tabular}{|c|c|c|c|c|c|c|c|}
\hline Matrix & $\begin{array}{l}\text { Chain of } \\
\text { Custody }\end{array}$ & $\begin{array}{l}\text { Work Agreement } \\
\text { Number }\end{array}$ & $\begin{array}{l}\text { Charge } \\
\text { Number }\end{array}$ & $\begin{array}{l}\text { Date } \\
\text { Sampled }\end{array}$ & $\begin{array}{l}\text { Date } \\
\text { Received }\end{array}$ & $\begin{array}{l}\text { Date } \\
\text { Needed }\end{array}$ & $\begin{array}{l}\text { Date } \\
\text { Approved }\end{array}$ \\
\hline -n-n & $\cdots+-$ & ----- & --- & - n-n- & -nen- & - & -........ \\
\hline GAS & 117931 & 81473 & $745 A 0221$ & $01 / 21 / 201000: 00$ & $01 / 21 / 2010 \quad 14: 30$ & $02 / 04 / 2010 \quad 23: 59$ & $02 / 17 / 2010 \quad 12: 30$ \\
\hline
\end{tabular}

Sample Description:

Location:

Sampler(s):

Customer comments:

Lab Comments:

Comment objects:

Proj Mgr: L P BURNETT (865) 576-9141

Sample Status: APPROVED

Sample Approver: J L MARSHALL/Chemist
Analytical Chemistry organization (Quality Services)

Page 1 of 1

P.o. Box 2009 oak Ridge, TN 378318244 (865) 574-2284

Lab Smp1 Id: A100250142

official Report

Cust Smp1 Id: HFIR water 150Mrad

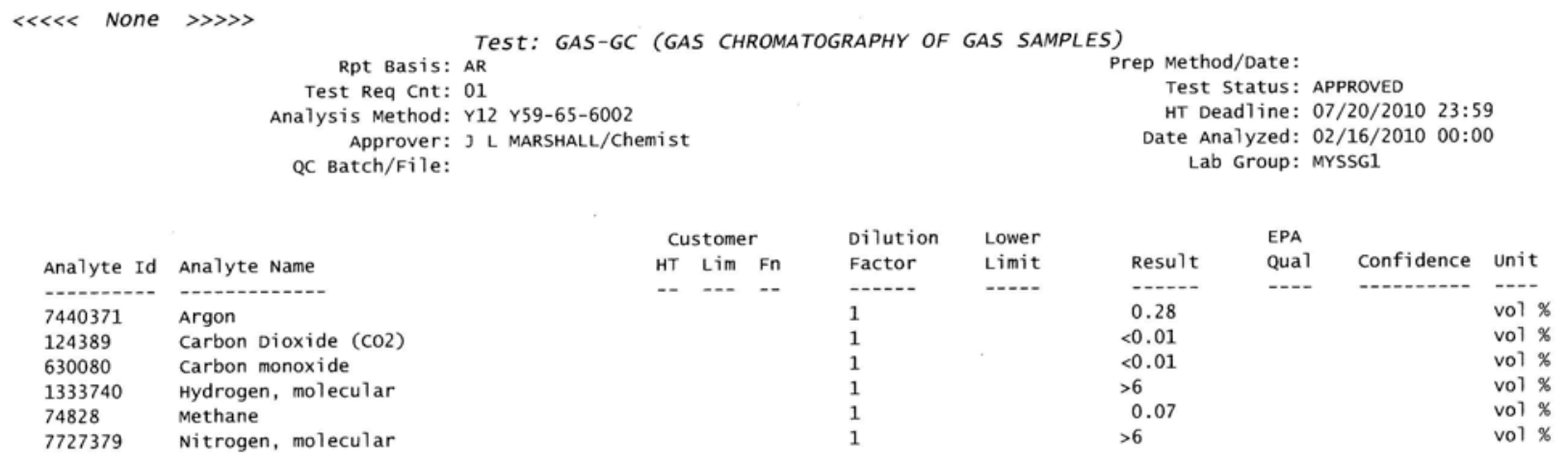

Sample Test Comments: $\mathrm{C} 2 \mathrm{H} 6=.01 \%[018053 \quad 02 / 17 / 201012: 17: 44]$

Comment objects:

Rpt Basis: AR Test Req Cnt: 01

Analysis Method: ASO Y/P65-6011

Approver: J L MARSHALL/Chemist

QC Batch/File:

Test: GAS-MS (Mass Spec. Analysis of Gas by VG 3001

Prep Method/Date:

Test Status: APPROVED

HT Deadline: 07/20/2010 23:59 Date Analyzed: 02/16/2010 00:00

Lab Group: MYSSG1

\begin{tabular}{|c|c|c|c|c|c|c|c|c|}
\hline \multicolumn{3}{|c|}{ Customer } & \multirow{2}{*}{$\begin{array}{l}\text { Dilution } \\
\text { Factor }\end{array}$} & \multirow{2}{*}{$\begin{array}{l}\text { Lower } \\
\text { Limit }\end{array}$} & \multirow[b]{2}{*}{ Result } & \multirow{2}{*}{$\begin{array}{l}\text { EPA } \\
\text { Qual }\end{array}$} & \multirow[b]{2}{*}{ Confidence } & \multirow[b]{2}{*}{ Unit } \\
\hline HT & Lim & $\mathrm{Fn}$ & & & & & & \\
\hline-- & -- & -- & - - & -...- & $-\cdots-$ & $\cdots$ & 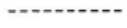 & --- \\
\hline & & & 1 & & 0.3 & & & vol $\%$ \\
\hline & & & 1 & & 32.04 & & & vol \% \\
\hline & & & 1 & & 56.5 & & & vol \% \\
\hline & & & 1 & & 0.06 & & & vol \% \\
\hline & & & 1 & & 10.75 & & & vol \% \\
\hline & & & 1 & & $<0.01$ & & & vol \% \\
\hline
\end{tabular}

Sample Test comments: $\mathrm{N} 20$ is <.01\% [018053 02/17/2010 $12: 15: 45]$

Comment objects:

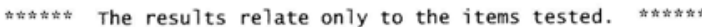

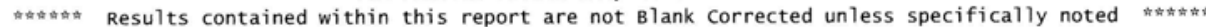

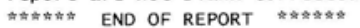

UNCLASSIFIED 


\section{UNCLASSIFIED}

LIMS 03.08 .048

$04 / 15 / 201014: 36$

Project: L MS MPO SCHUH GAS

Customer: L P BURNETT B B dg 9995 MS-8189 (865) 576-9141

\begin{tabular}{|c|c|c|c|c|c|c|c|}
\hline Matrix & $\begin{array}{l}\text { Chain of } \\
\text { Custody }\end{array}$ & $\begin{array}{l}\text { Work Agreement } \\
\text { Number }\end{array}$ & $\begin{array}{l}\text { Charge } \\
\text { Number }\end{array}$ & $\begin{array}{l}\text { Date } \\
\text { Sampled }\end{array}$ & $\begin{array}{l}\text { Date } \\
\text { Received }\end{array}$ & $\begin{array}{l}\text { Date } \\
\text { Needed }\end{array}$ & $\begin{array}{l}\text { Date } \\
\text { Approved }\end{array}$ \\
\hline---- & ------ & $\cdots$ & - - & - - & - n- & $-\cdots-$ & - - \\
\hline GAS & 117946 & 81473 & $745 A 0221$ & 03/31/2010 07:30 & $04 / 01 / 2010 \quad 14: 15$ & $04 / 15 / 2010 \quad 23: 59$ & $04 / 15 / 2010 \quad 14: 36$ \\
\hline
\end{tabular}

Customer Comments:

Lab Comments: Need stainless bottles by April 26th for time sensitive sample grab. [018254 04/01/2010 15:50:56]

Comment objects:

Proj Mgr: L P BURNETT (865) 576-9141
Analytical Chemistry organization (Quality Services)

P.o. Box 2009 oak Ridge, TN 378318244 (865) 574-2284

Page 1 of 1

official Report

Lab Smpl Id: A100910400

Cust Smpl Id: HFIR oldwater 50Mrad

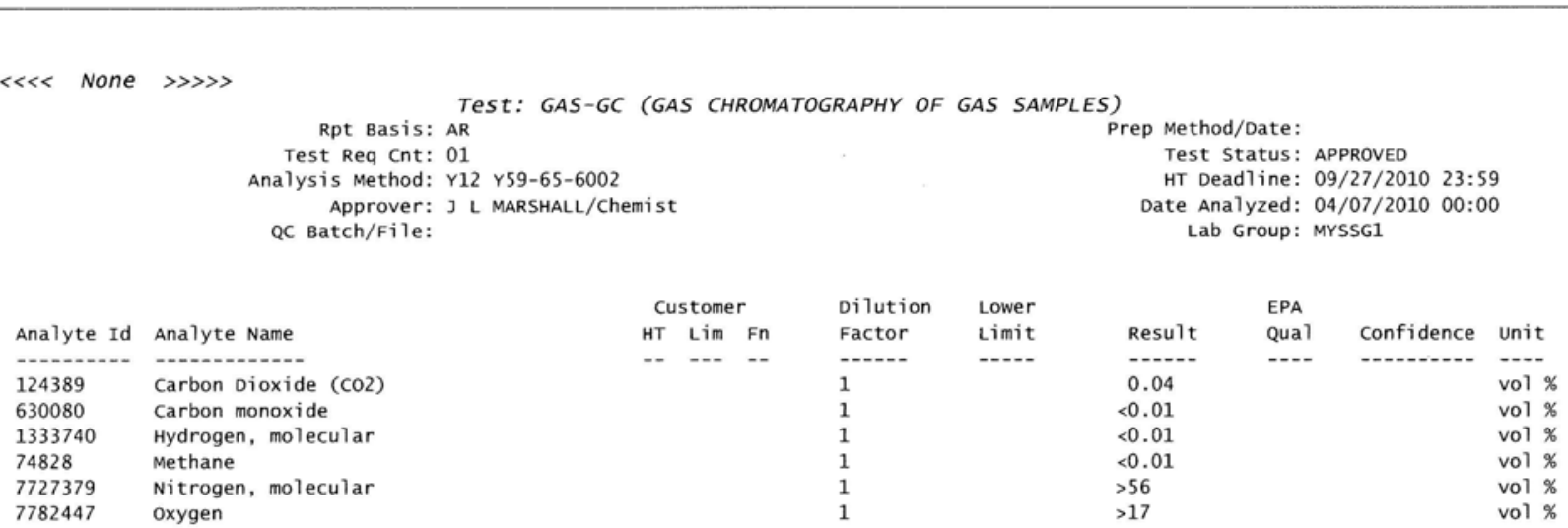

Comment objects: Test: GAS-MS (Mass Spec. Analysis of Gas by VG 3001)

Rpt Basis: AR Test Req Cnt: 01

Analys is Method: ASO Y/P65-6011

Approver: J L MARSHALL/Chemist

QC Batch/File:

Test Status: APPROVED

HT Deadline: 09/27/2010 23:59

Date Analyzed: 04/07/2010 00:00

Lab Group: MYSSG1

\begin{tabular}{|c|c|}
\hline Analyte Id & Analyte Name \\
\hline 7440371 & Argon \\
\hline 7440597 & Helium \\
\hline 1333740 & Hydrogen, molecular \\
\hline 74828 & Methane \\
\hline 7727379 & Nitrogen, molecular \\
\hline 7782447 & oxygen \\
\hline
\end{tabular}

Customer Dilution Lower

HT Lim Fn Factor Limit

(1

Limit

EPA

\begin{tabular}{llll} 
Result & Qua) & Confidence & Unit \\
\hline 0.94 & & & -1 \\
$<0.01$ & & & vol $\%$ \\
0.01 & & & vol $\%$ \\
0.1 & & & vol $\%$ \\
77.34 & & & vol $\%$ \\
21.56 & & & vol $\%$ \\
& & & vol $\%$
\end{tabular}

Comment objects:

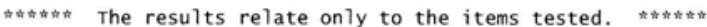

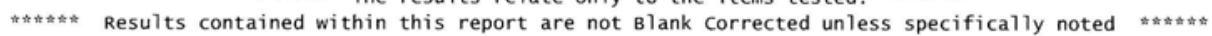

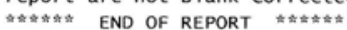

UNCLASSIFIED 


\section{UNCLASSIFIED}

LIMS 03.08 .048

03/24/2010 10:47

Project: L MS MPO SCHUH GAS

Customer: L P BURNETT Bldg 9995 MS-8189 (865) 576-9141

\begin{tabular}{llcl} 
Matrix & $\begin{array}{l}\text { Chain of } \\
\text { Custody }\end{array}$ & $\begin{array}{l}\text { work Agreement } \\
\text { Number }\end{array}$ & $\begin{array}{l}\text { Charge } \\
\text { Number }\end{array}$ \\
\hdashline GAS & 117945 & -81473 & -7450221 \\
& Sample Description: GAS \\
Location: \\
Sampler(s):
\end{tabular}

ytical Chemistry organization (Quality Services)

P.O. Box 2009 oak Ridge, TN 378318244 (865) 574-2284

Proj Mgr: L P BURNETT (865) 576-9141

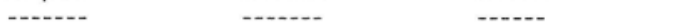

official Report

Page 1 of 1

Lab Smpl Id: A100690173

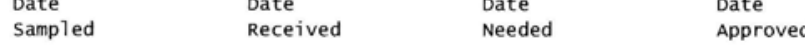

03/09/2010 08:00 03/09/2010 $14: 10 \quad 03 / 23 / 2010 \quad 23: 59 \quad 03 / 24 / 2010 \quad 10: 47$

Sample Status: APPROVED

Sample Approver: J L MARSHALL/Chemist

Customer Comments:

Lab comments:

Comment objects:

$\ll<<$ None $\gg>>>$

Rpt Basis: AR

Test: GAS-GC (GAS CHROMATOGRAPHY OF GAS SAMPLES) Test Req Cnt: 01 Analysis Method: Y12 Y59-65-6002

Approver: J L MARSHALL/Chemist QC Batch/File:

Prep Method/Date:

Test Status: APPROVED

HT Deadline: 09/05/2010 23:59

Date Analyzed: 03/22/2010 00:00

Lab Group: MYSSG1

$\begin{array}{lllllrl} & & \text { Customer } & \text { Dilution } & \text { Lower } & \text { EPA } \\ \text { Analyte Id } & \text { Analyte Name } & \text { HT Lim Fn } & \text { Factor } & \text { Limit } & \text { Result } & \text { Qual } \\ \text { Confidence Unit }\end{array}$

Comment objects:

Rpt Basis: AR

Test: GAS-MS (Mass Spec. Analysis of Gas by VG 3001)

Test Req

Analysis Method: ASO $\mathrm{Y} / \mathrm{P} 65-6011$

QC Batch/File:

Prep Method/Date:

Test Status: APPROVED

Date Analyzed: 03/22/2010 00:00

Lab Group: MYSSG1

\begin{tabular}{ll} 
Analyte Id & Ana7yte Name \\
\hline 7440371 & Argon \\
7440597 & Helium \\
1333740 & Hydrogen, molecular \\
74828 & Methane \\
7727379 & Nitrogen, molecular \\
7782447 & Oxygen
\end{tabular}

\begin{tabular}{|c|c|c|c|c|c|c|c|c|}
\hline \multicolumn{3}{|c|}{ Customer } & \multirow{2}{*}{$\begin{array}{l}\text { Dilution } \\
\text { Factor }\end{array}$} & \multirow{2}{*}{$\begin{array}{l}\text { Lower } \\
\text { Limit }\end{array}$} & & \multicolumn{2}{|l|}{ EPA } & \multirow[b]{2}{*}{ Unit } \\
\hline$H T$ & Lim & $\mathrm{Fn}$ & & & Result & Qua 1 & Confidence & \\
\hline-- & $\cdots$ & -- & --- & $\cdots$ & -n- & $\cdots$ & - - - & -- \\
\hline & & & 1 & & 0.9 & & & vol $\%$ \\
\hline & & & 1 & & $<0.1$ & & & vol $x$ \\
\hline & & & 1 & & 1.13 & & & vol $?$ \\
\hline & & & 1 & & 0.01 & & & vol? \\
\hline & & & 1 & & 79.38 & & & vol $x$ \\
\hline & & & 1 & & 18.4 & & & vol $x$ \\
\hline
\end{tabular}

Sample Test Comments: $\mathrm{N} 2 \mathrm{O}=.16 \%[018053 \quad 03 / 24 / 2010 \quad 10: 46: 37]$

Comment objects:

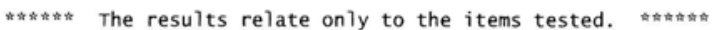

Results contained within this report are not Blank corrected unless specifically noted

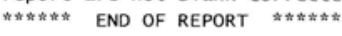

UNCLASSIFIED 


\section{UNCLASSIFIED}

LIMS 03.08 .048

03/24/2010 10:46

Project: L MS MPO SCHUH GAS

Customer: L. P BURNETT Bldg 9995 MS-8189 (865) 576-9141

\begin{tabular}{|c|c|c|c|}
\hline Matrix & $\begin{array}{l}\text { Chain of } \\
\text { Custody }\end{array}$ & $\begin{array}{l}\text { work Agreement } \\
\text { Number }\end{array}$ & $\begin{array}{l}\text { Charge } \\
\text { Number }\end{array}$ \\
\hline - & - - & ----- & $-\cdots$ \\
\hline GAS & 117945 & 81473 & $745 A 0221$ \\
\hline
\end{tabular}

Sampled

03/09/2010 08:00
Date
Received

03/09/2010 $14: 10$
Page 1 of 1

Lab Smp1 Id: A100690172

Customer comments:

Lab comments:

Comment objects:

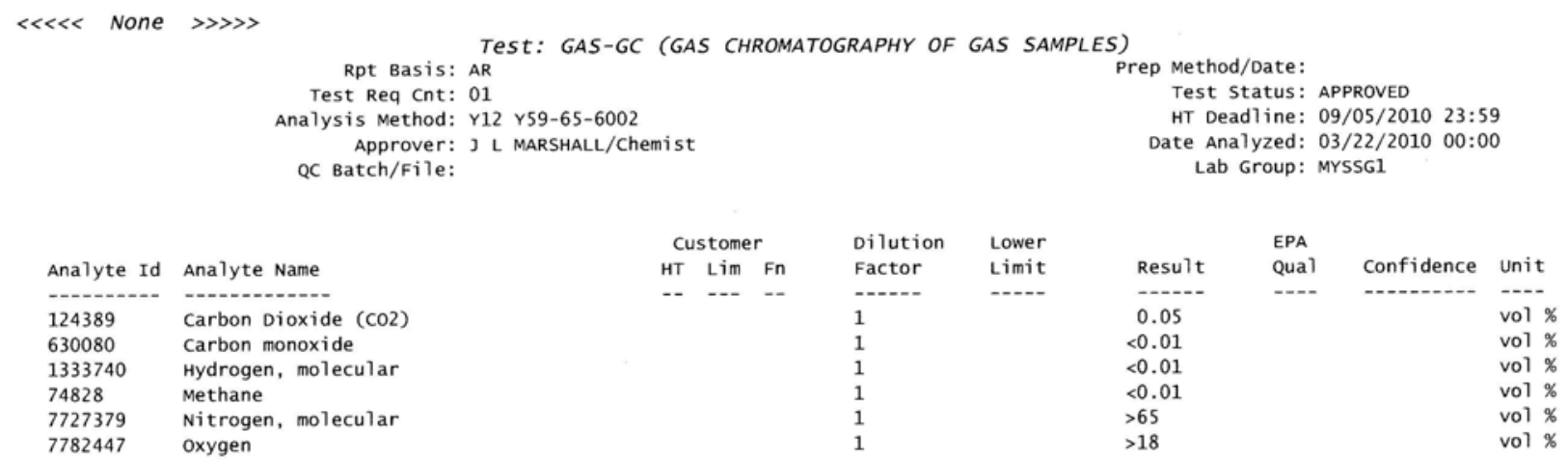

Comment objects:

Test: GAS-MS (Mass Spec. Analysis of Gas by VG 3001)

Rpt Basis: AR Test Req Cnt: 01

Analysis Method: ASO Y/P65-6011

Approver: J L MARSHALL/Chemist

QC Batch/File:

Prep Method/Date:

Test Status: APPROVED

HT Deadline: 09/05/2010 23:59

Date Analyzed: 03/22/2010 00:00

Lab Group: MYSSG1

\begin{tabular}{|c|c|}
\hline Analyte Id & Analyte Name \\
\hline - - & - \\
\hline 7440371 & Argon \\
\hline 1333740 & Hydrogen, molecular \\
\hline 74828 & Methane \\
\hline 7727379 & Nitrogen, molecular \\
\hline 7782447 & oxygen \\
\hline
\end{tabular}

\begin{tabular}{|c|c|c|c|c|c|c|c|c|}
\hline \multicolumn{3}{|c|}{ Customer } & \multirow{2}{*}{$\begin{array}{l}\text { Dilution } \\
\text { Factor }\end{array}$} & \multirow{2}{*}{$\begin{array}{l}\text { Lower } \\
\text { Limit }\end{array}$} & & \multicolumn{2}{|l|}{ EPA } & \multirow[b]{2}{*}{ Unit } \\
\hline HT & Lim & $\mathrm{Fn}$ & & & Result & Qual & Confidence & \\
\hline-- & $\cdots$ & -- & -..... & -...- & $-\cdots-$ & $\cdots$ & -...-..... & --- \\
\hline & & & 1 & & 0.77 & & & vol $\%$ \\
\hline & & & 1 & & 43.34 & & & vol \% \\
\hline & & & 1 & & 0.01 & & & vol \% \\
\hline & & & 1 & & 50.1 & & & vol \% \\
\hline & & & 1 & & 5.3 & & & vol \% \\
\hline
\end{tabular}

Sample Test Comments: $\mathrm{N} 20=.31 \%[018053 \quad 03 / 24 / 2010 \quad 10: 45: 21]$

Comment objects:

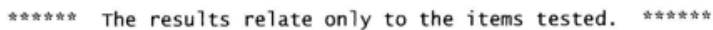

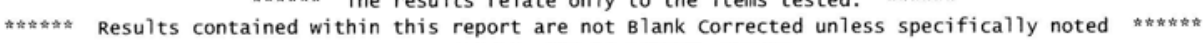

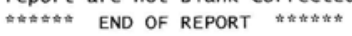

UNCLASSIFIED 


\section{UNCLASSIFIED}

LIMS 03.08.048

03/08/2010 13:33

Project: L MS MPO SCHUH GAS

Customer: L P BURNETT Bldg 9995 MS-8189 (865) 576-9141

\begin{tabular}{llll} 
Matrix & $\begin{array}{l}\text { Chain of } \\
\text { Custody }\end{array}$ & $\begin{array}{l}\text { Work Agreement } \\
\text { Number }\end{array}$ & $\begin{array}{l}\text { Charge } \\
\text { Number }\end{array}$ \\
\hdashline GAS & 117935 & -81473 & -745 A0221 \\
-3 & Sample Description: Nitric Acid \\
Location: \\
Sampler(s):
\end{tabular}

Sampled

-....--

02/23/2010 00:00
Date
Received
$-02 / 23 / 2010 \quad 14: 30$

Date

Needed

$02 / 23 / 201014: 30$

03/09/2010 23:59 03/08/2010 13:33

Page 1 of 1

Lab Smpl Id: A100550033

Sample Status: APPROVED

Sample Approver: J L MARSHALL/Chemist

Customer comments:

Lab comments:

Comment objects:

$\ll<<$ None $\gg \gg>$ Rpt Basis: AR
Test Req Cnt: 01 Test: GAS-GC (GAS CHROMATOGRAPHY OF GAS SAMPLES)

Analysis Method: Y12 Y59-65-6002

Approver: J L MARSHALL/Chemist

QC Batch/File:

Prep Method/Date:

Test Status: APPROVED

HT Deadline: 08/22/2010 23:59

Date Analyzed: 03/04/2010 00:00

Lab Group: MYSSG1

\begin{tabular}{|c|c|c|c|c|c|c|c|c|c|c|}
\hline & & & stome & & Dilution & Lower & & EPA & & \\
\hline nalyte Id & Analyte Name & HT & Lim & $\mathrm{Fn}$ & Factor & Limit & Result & Qual & Confidence & Unit \\
\hline . & $-0-1-2-1-1$ & -- & -- & -- & -.... & $\ldots$ & $\ldots$ & $\ldots$ & & -- \\
\hline
\end{tabular}

Sample Test Comments: There was no sample in this section of tube. [018053 03/08/2010 13:22:31]

Comment objects:

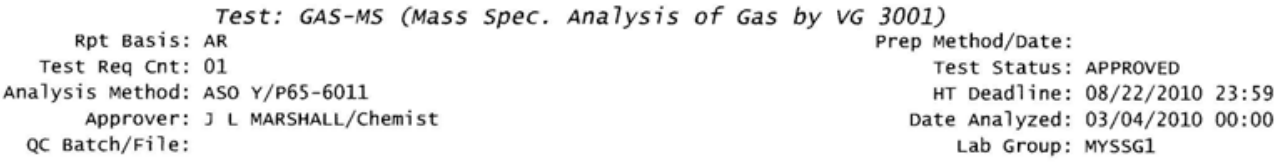

Approver: I L MARSHALL/Chemist

QC Batch/File:

Prep Method/Date:

Test Status: APPROVED

HT Deadline: 08/22/2010 23:59

Date Analyzed: 03/04/2010 00:00

Lab Group: MYSSG1

\begin{tabular}{ll} 
Analyte Id & Analyte Name \\
\hline 7440371 & Argon \\
7440597 & Helium \\
1333740 & Hydrogen, molecular \\
74828 & Methane \\
7727379 & Nitrogen, molecular \\
7782447 & oxygen
\end{tabular}

Customer
HT Lim Fn

$\begin{array}{ll}\text { Dilution } & \text { Lower } \\ \text { Factor } & \text { Limit } \\ -1 & \\ 1 & \\ 1 & \\ 1 & \\ 1 & \\ 1 & \end{array}$

Result
-0.26
0.02
16.72
$<0.01$
47.72
4.01

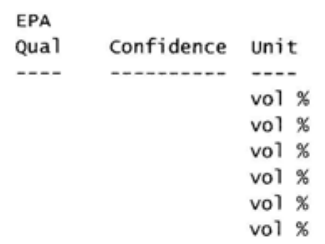

Sample Test Comments: $\mathrm{N} 2 \mathrm{O}+\mathrm{CO} 2=31.25 \%[018053 \quad 03 / 08 / 2010 \quad 13: 23: 13]$

Comment objects: 


\section{UNCLASSIFIED}

LIMS 03.08 .048

03/24/2010 10:43

Project: L MS MPO SCHUH GAS

Customer: L P BURNETT Bldg 9995 MS-8189 (865) 576-9141

\begin{tabular}{|c|c|c|c|c|c|c|c|}
\hline Matrix & $\begin{array}{l}\text { Chain of } \\
\text { Custody }\end{array}$ & $\begin{array}{l}\text { Work Agreement } \\
\text { Number }\end{array}$ & $\begin{array}{l}\text { Charge } \\
\text { Number }\end{array}$ & $\begin{array}{l}\text { Date } \\
\text { Sampled }\end{array}$ & $\begin{array}{l}\text { Date } \\
\text { Received }\end{array}$ & $\begin{array}{l}\text { Date } \\
\text { Needed }\end{array}$ & $\begin{array}{l}\text { Date } \\
\text { Approved }\end{array}$ \\
\hline$-\cdots-$ & --n- & 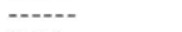 & --.-- & $\cdots$ & -1-n-s- & ----- & $-\cdots$ \\
\hline GAS & 117945 & 81473 & $745 A 0221$ & 03/09/2010 08:00 & 03/09/2010 $14: 10$ & $03 / 23 / 2010 \quad 23: 59$ & $03 / 24 / 201010: 43$ \\
\hline
\end{tabular}

Customer Comments:

Lab comments:

Comment objects:
Analytical Chemistry Organization (Quality Services)

P.O. Box 2009 oak Ridge, TN 378318244 (865) 574-2284

official Report

Proj Mgr: L P BURNETT (865) 576-9141
Page 1 of 1

Lab Smpl Id: A100690171 HFIR NITRIC $250 \mathrm{Mrad}$

None $\gg>>>$

《<< None $\gg \gg \gg>$ Test: GAS-GC (GAS CHROMATOGRAPHY OF GAS SAMPLES)

Rpt Basis: AR Test Req Cnt: 01 Analysis Method: Y12 Y59-65-6002

Approver: J L MARSHALL/Chemist QC Batch/File:

Prep Method/Date:

Test Status: APPROVED

HT Deadline: 09/05/2010 23:59

Date Analyzed: 03/22/2010 00:00

Lab Group: MYSSG1

\begin{tabular}{|c|c|c|c|c|c|c|c|c|}
\hline & stom & & Dilution & Lower & & EPA & & \\
\hline HT & Lim & $\mathrm{Fn}$ & Factor & Limit & Result & Qual & Confidence & Unit \\
\hline-- & -- & -- & - & --..- & -1.-.- & $\cdots$ & -....... & -2 \\
\hline & & & 1 & & $>2$ & & & vol \% \\
\hline & & & 1 & & 0.21 & & & vol \% \\
\hline & & & 1 & & $>4$ & & & vol \% \\
\hline & & & 1 & & $<0.01$ & & & vol \% \\
\hline & & & 1 & & $>5$ & & & vol \% \\
\hline & & & 1 & & 0.17 & & & vol \% \\
\hline
\end{tabular}

Comment objects:

Test: GAS-MS (Mass Spec. Analysis of Gas by VG 3001)

Rpt Basis: AR Test Req Cnt: 01 Analysis Method: ASO Y/P65-6011

Approver: J L MARSHALL/Chemist QC Batch/File:

Prep Method/Date:

Test Status: APPROVED

HT Deadline: 09/05/2010 23:59

Date Analyzed: 03/22/2010 00:00 Lab Group: MYSSG1

$\begin{array}{ll}\text { Analyte Id } & \text { Analyte Name } \\ 7440371 & \text { Argon } \\ 7440597 & \text { Helium } \\ 1333740 & \text { Hydrogen, molecular } \\ 74828 & \text { Methane } \\ 7727379 & \text { Nitrogen, molecular } \\ 7782447 & \text { oxygen }\end{array}$

\begin{tabular}{|c|c|c|c|c|c|c|c|c|}
\hline $\mathrm{Cu}$ & stome & & Dilution & Lower & & EPA & & \\
\hline HT & Lim & $\mathrm{Fn}$ & Factor & Limit & Result & Qual & Confidence & Unit \\
\hline-- & $\cdots$ & -- & ----- & -.... & --1-- & $\cdots$ & 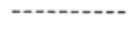 & --- \\
\hline & & & 1 & & 0.06 & & & vol ? \\
\hline & & & 1 & & $<0.1$ & & & vol $x$ \\
\hline & & & 1 & & 7.7 & & & vol $x$ \\
\hline & & & 1 & & $<0.01$ & & & vol $x$ \\
\hline & & & 1 & & 22.39 & & & vol $x$ \\
\hline & & - & 1 & & 0.06 & & & vol $x$ \\
\hline
\end{tabular}

Sample Test Comments: $N 20=69.68 \%[018053 \quad 03 / 24 / 2010 \quad 10: 42: 38]$

Comment objects:

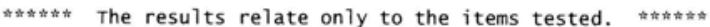

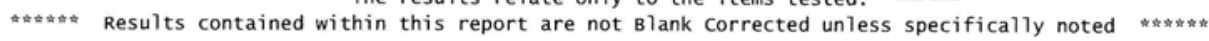

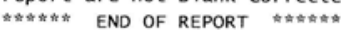

UNCLASSIFIED 


\section{UNCLASSIFIED}

LIMS 03.08 .048

04/15/2010 14:33

Project: L MS MPO SCHUH GAS

$\begin{array}{llc}\text { Matrix } & \begin{array}{l}\text { Chain of } \\ \text { Custody }\end{array} & \begin{array}{l}\text { Work Agreement } \\ \text { Number }\end{array} \\ - \text { GAS } & 117946 & -81473 \\ & \text { Sample Description: GRAB } \\ \text { Location: } \\ \text { Sampler(s): }\end{array}$

Analytical Chemistry Organization (Quality Services)

P.O. Box 2009 oak Ridge, TN 378318244 (865) 574-2284

Page 1 of 1
Proj Mgr: L P BURNETT (865) 576-9141
Lab Smpl Id: A100910398

Cust Smp 1 Id: HFIR Nitric 200Mrad

\begin{tabular}{|c|c|c|c|c|}
\hline $\begin{array}{l}\text { Charge } \\
\text { Number }\end{array}$ & Date & Date & Date & $\begin{array}{l}\text { Date } \\
\text { Approved }\end{array}$ \\
\hline - & -...... & -and & 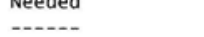 & Approved \\
\hline $745 A 0221$ & $03 / 31 / 2010 \quad 07: 30$ & $04 / 01 / 2010 \quad 14: 15$ & $04 / 15 / 2010 \quad 23: 59$ & $04 / 15 / 2010 \quad 14: 33$ \\
\hline
\end{tabular}

Customer comments:

Lab Comments: Need stainless bottles by April 26th for time sensitive sample grab. [018254 04/01/2010 15:50:56]

comment objects:

$\ll<<$ None $\gg>>>$

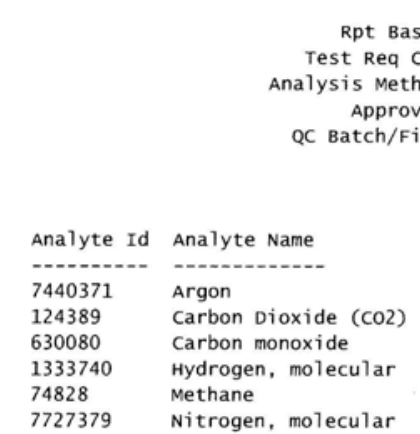

Test: GAS-GC (GAS CHROMATOGRAPHY OF GAS SAMPLES)

Rpt Basis: AR

Method: Y12 Y59-65-6002

Approver: J L MARSHALL/Chemist

Test Status: APPROVED

HT Deadline: 09/27/2010 23:59

Date Ana7yzed: 04/07/2010 00:00

Lab Group: MYSSG1

\begin{tabular}{ll} 
Analyte Id & Analyte Name \\
\hline 7440371 & Argon \\
124389 & Carbon Dioxide (CO2) \\
630080 & Carbon monoxide \\
1333740 & Hydrogen, molecular \\
74828 & Methane \\
7727379 & Nitrogen, molecular
\end{tabular}

\begin{tabular}{|c|c|c|c|c|c|c|c|}
\hline Custom & & Dilution & Lower & & EPA & & \\
\hline Lim & $\mathrm{Fn}$ & Factor & Limit & Result & Qual & Confidence & Unit \\
\hline-- & -- & ---- & - & $---\cdot-$ & $\cdots$ & 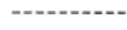 & --- \\
\hline & & 1 & & 0.13 & & & vol $\%$ \\
\hline & & 1 & & $>11$ & & & vol $\%$ \\
\hline & & 1 & & 0.45 & & & vol $\%$ \\
\hline & & 1 & & $>12$ & & & vol $\%$ \\
\hline & & 1 & & 0.002 & & & vol \% \\
\hline & & 1 & & $>17$ & & & vol $\%$ \\
\hline
\end{tabular}

Comment objects: Rpt Basis: AR Test: GAS-MS (Mass Spec. Analysis of Gas by VG 3001) Test Req Cnt: 01 Analysis Method: ASO Y/P65-6011 Approver: J L MARSHALL/Chemist QC Batch/File:

Prep Method/Date:

Test Status: APPROVED

HT Deadline: 09/27/2010 23:59 Date Analyzed: 04/07/2010 00:00 Lab Group: MYSSG1

\begin{tabular}{ll} 
Analyte Id & Analyte Name \\
\hdashline 7440371 & Argon \\
124389 & Carbon Dioxide (CO2) \\
7440597 & Helium \\
1333740 & $\begin{array}{l}\text { Hydrogen, molecular } \\
74828\end{array}$ \\
7727379 & Methane \\
7782447 & Nitrogen, molecular \\
& Oxygen
\end{tabular}

\begin{tabular}{|c|c|c|c|c|c|c|c|c|}
\hline & tome & & Dilution & Lower & & EPA & & \\
\hline HT & Lim & $\mathrm{Fn}$ & Factor & Limit & Result & Qua1 & Confidence & Unit \\
\hline-- & -- & -- & - & -...- & $-\ldots-$ &.-- & $-\cdots$ & -... \\
\hline & & & 1 & & 0.12 & & & vol $\%$ \\
\hline & & & 1 & & 24.24 & & & vol $\%$ \\
\hline & & & 1 & & $<0.1$ & & & vol \% \\
\hline & & & 1 & & 24.83 & & & vol \% \\
\hline & & & 1 & & 0.02 & & & vol $\%$ \\
\hline & & & 1 & & 29.63 & & & vol $\%$ \\
\hline & & & 1 & & 0.03 & & & vol \% \\
\hline
\end{tabular}

Comment objects:

\section{UNCLASSIFIED}




\section{UNCLASSIFIED}

LIMS 03.08 .048

$04 / 15 / 201014: 34$

Project: L MS MPO SCHUH GAS
Analytical Chemistry Organization (Quality Services)

P.o. Box 2009 oak Ridge, TN 378318244 (865) 574-2284 official Report

Proj Mgr: L P BURNETT (865) 576-9141
Page 1 of 1

Lab Smpl Id: A100910399

\begin{tabular}{|c|c|c|c|c|c|c|c|}
\hline Matrix & $\begin{array}{l}\text { Chain of } \\
\text { Custody }\end{array}$ & $\begin{array}{l}\text { Work Agreement } \\
\text { Number }\end{array}$ & $\begin{array}{l}\text { Charge } \\
\text { Number }\end{array}$ & $\begin{array}{l}\text { Date } \\
\text { Sampled }\end{array}$ & $\begin{array}{l}\text { Date } \\
\text { Received }\end{array}$ & $\begin{array}{l}\text { Date } \\
\text { Needed }\end{array}$ & $\begin{array}{l}\text { Date } \\
\text { Approved }\end{array}$ \\
\hline --- & --- & -...- & $-\cdots-$ & $-\ldots-$ & $-\cdots-$ &.--- & --n-- \\
\hline GAS & 117946 & 81473 & $745 A 0221$ & $03 / 31 / 2010 \quad 07: 30$ & $04 / 01 / 2010 \quad 14: 15$ & $04 / 15 / 2010 \quad 23: 59$ & $04 / 15 / 2010 \quad 14: 34$ \\
\hline
\end{tabular}

Customer Comments:

Lab Comments: Need stainless bottles by April 26th for time sensitive sample grab. [018254 04/01/2010 15:50:56]

Comment objects:

$\ll<<$ None $\gg>>>$

Test: GAS-GC (GAS CHROMATOGRAPHY OF GAS SAMPLES)

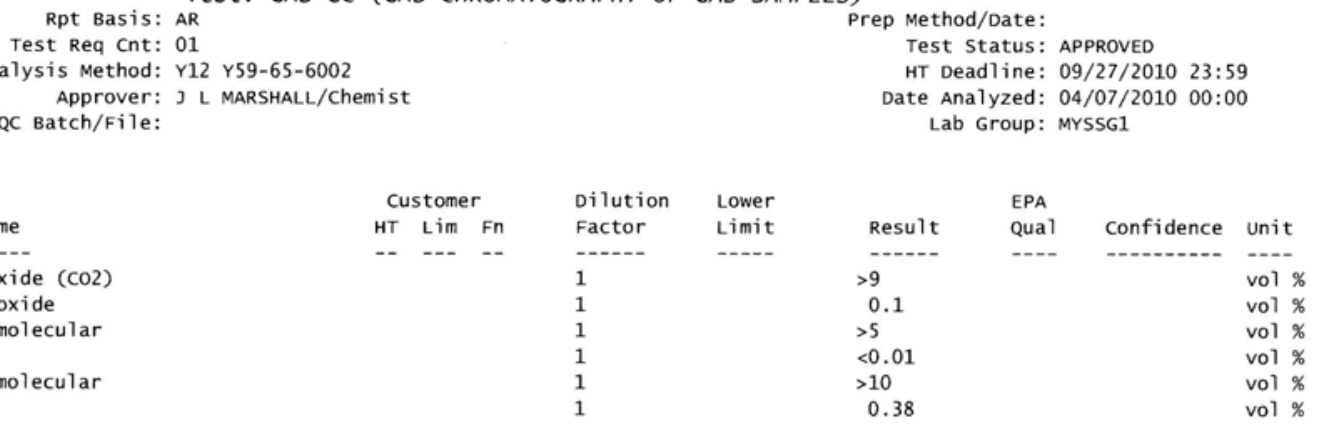

Comment objects:

Rpt Basis: AR

Test Req Cnt: 01

Analysis Method: ASO $\mathrm{Y} / \mathrm{P} 65-6011$

Approver: J L MARSHALL/Chemist

QC Batch/File:

Test: GAS-MS (Mass Spec. Analysis of Gas by VG 3001)

Prep Method/Date:

Test Status: APPROVED

HT Deadline: 09/27/2010 23:59

Date Analyzed: 04/07/2010 00:00

Lab Group: MYSSG1

\begin{tabular}{ll} 
Analyte Id & Analyte Name \\
\hdashline 7440371 & Argon \\
124389 & Carbon Dioxide (CO2) \\
7440597 & Helium \\
1333740 & Hydrogen, molecular \\
74828 & Methane \\
7727379 & Nitrogen, molecular \\
7782447 & oxygen
\end{tabular}

Customer Dilution Lower HT Lim Fn Factor Limit

$\begin{array}{llll} & \text { EPA } & & \\ \text { Result } & \text { Qual } & \text { Confidence } & \text { Unit } \\ -0.46 & & & -1 \% \\ 19.8 & & & \text { vol } \% \\ 0.27 & & \text { vol } \% \\ 6.52 & & \text { vol } \% \\ 0.02 & & \text { vol } \% \\ 55.7 & & & \text { vol } \% \\ 9.15 & & \text { vol } \% \\ & & \text { vol } \%\end{array}$

Comment objects :

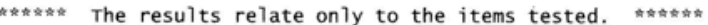

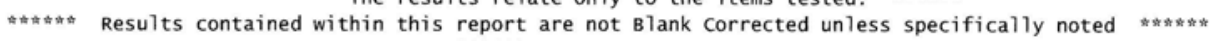

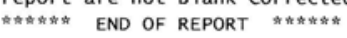

UNCLASSIFIED 
APPENDIX B. ANALYTICAL RESULTS FOR LIQUID SAMPLES FROM RADIOLYSIS TESTS 



\section{UNCLASSIFIED}

LIMS 03.08 .048

05/18/2010 14:02
Analytical Chemistry Organization (Quality Services)

P.o. Box 2009 oak Ridge, TN 378318244 (865) 574-2284
Page 1 of 3

Lab Smp 1 Id: A101230505

Project: L MS MPO SCHUH SVOA

Cust Smp1 Id: Co-60 300 SRS

Proj Mgr: L P BURNETT (865) 576-9141

Customer: L P BURNETT B1dg 9995 MS-8189 (865) 576-9141

\begin{tabular}{|c|c|c|c|c|c|c|c|}
\hline Matrix & $\begin{array}{l}\text { Chain of } \\
\text { Custody }\end{array}$ & $\begin{array}{l}\text { Work Agreement } \\
\text { Number }\end{array}$ & $\begin{array}{l}\text { Charge } \\
\text { Number }\end{array}$ & $\begin{array}{l}\text { Date } \\
\text { Sampled }\end{array}$ & $\begin{array}{l}\text { Date } \\
\text { Received }\end{array}$ & $\begin{array}{l}\text { Date } \\
\text { Needed }\end{array}$ & $\begin{array}{l}\text { Date } \\
\text { Approved }\end{array}$ \\
\hline ----- & ------ & -..-- & ---- & - n-- & ---.-- & - n- & - n-n- \\
\hline LIQUID & 117947 & 81473 & $745 A 0221$ & $04 / 28 / 2010 \quad 12: 30$ & $05 / 03 / 2010 \quad 15: 19$ & $05 / 17 / 2010 \quad 23: 59$ & $05 / 18 / 2010 \quad 14: 02$ \\
\hline
\end{tabular}

Sample Description:

Location:

Sample Status: APPROVED

Sampler(s):

Sample Approver: J T HOFFARTH/Data Approver

Customer comments:

Lab comments:

Comment objects:

$\ll<<$ Inorganic $\gg \gg>>$

Test: Toc (Total Organic Carbon, 415.1)

: 01

Analysis Method: EPA 415.11983 (Y50-AC-65-7314)

Approver:

QC Batch/File:
Prep Method/Date:

Test Status: CANCELLED

HT Deadline: 05/26/2010 23:59

Date Analyzed:

Lab Group: IAWETC

Sample Test comments:

Change to TOC 9060 [027645 05/06/2010 06:15:08]

$\ll<<$ organic $\gg \gg \gg$

Rpt Basis: $A R$ Test Req Cnt: 01

Analysis Method: SW846 8270C (Y50-AC-65-7316)

Approver: J T HOFFARTH/Data Approver

QC Batch/File: QC10138016/5385/53858
Prep Method/Date: Sw846 3580A 05/05/2010 15:00 Test Status: APPROVED

HT Deadline: $06 / 14 / 2010 \quad 23: 59$

Date Analyzed: 05/13/2010 13:53

Lab Group: OYGCMS

\begin{tabular}{ll} 
Analyte Id & Analyte Name \\
\hline 120821 & 1,2,4-Trichlorobenzene \\
95501 & 1,2-Dichlorobenzene \\
122667 & 1,2-Diphenylhydrazine \\
541731 & 1,3-Dichlorobenzene \\
106467 & 1,4-Dichlorobenzene \\
95954 & 2,4,5-Trichlorophenol \\
88062 & 2,4,6-Trichlorophenol \\
120832 & 2,4-Dichlorophenol \\
105679 & 2,4-Dimethylphenol \\
51285 & 2,4-Dinitrophenol \\
121142 & 2,4-Dinitrotoluene \\
606202 & 2,6-Dinitrotoluene \\
91587 & 2-Chloronaphthalene \\
95578 & 2-Chlorophenol \\
534521 & 2-Methyl-4,6-dinitrophenol \\
91576 & 2-Methylnaphthalene \\
95487 & 2-Methylphenol \\
88744 & 2-Nitrobenzenamine \\
88755 & 2-Nitrophenol \\
91941 & 3,3'-Dichlorobenzidine \\
N2799 & 3- and 4- Methylphenol \\
99092 & 3-Nitrobenzenamine \\
101553 & 4-Bromophenyl phenyl ether \\
59507 & 4-Chloro-3-methylphenol \\
106478 & 4-Chlorobenzenamine \\
7005723 & 4-Chlorophenylphenyl ether \\
100016 & 4-Nitrobenzenamine \\
100027 & 4-Nitrophenol \\
83329 & Acenaphthene \\
208968 & Acenaphthylene \\
62533 & Aniline \\
120127 & Anthracene \\
100516 & Benzenemethanol \\
92875 & Benzidine \\
56553 & Benzo(a)anthracene \\
&
\end{tabular}

\begin{tabular}{|c|c|c|c|c|}
\hline & stome & & Dilution & Lower \\
\hline HT & Lim & $\mathrm{Fn}$ & Factor & Limit \\
\hline-- & -- & -- & ----- & --- \\
\hline & & & 1 & 99 \\
\hline & & & 1 & 110 \\
\hline & & & 1 & 59 \\
\hline & & & 1 & 170 \\
\hline & & & 1 & 180 \\
\hline & & & 1 & 190 \\
\hline & & & 1 & 100 \\
\hline & & & 1 & 98 \\
\hline & & & 1 & 99 \\
\hline & & & 1 & 140 \\
\hline & & & 1 & 130 \\
\hline & & & 1 & 97 \\
\hline & & & 1 & 87 \\
\hline & & & 1 & 100 \\
\hline & & & 1 & 110 \\
\hline & & & 1 & 66 \\
\hline & & & 1 & 98 \\
\hline & & & 1 & 110 \\
\hline & & & 1 & 81 \\
\hline & & & 1 & 49 \\
\hline & & & 1 & 120 \\
\hline & & & 1 & 220 \\
\hline & & & 1 & 66 \\
\hline & & & 1 & 55 \\
\hline & & & 1 & 100 \\
\hline & & & 1 & 67 \\
\hline & & & 1 & 110 \\
\hline & & & 1 & 100 \\
\hline & & & 1 & 91 \\
\hline & & & 1 & 100 \\
\hline & & & 1 & 40 \\
\hline & & & 1 & 52 \\
\hline & & & 1 & 61 \\
\hline & & & 1 & 120 \\
\hline & & & 1 & 57 \\
\hline
\end{tabular}

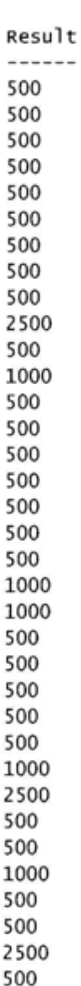

\begin{tabular}{|c|c|c|}
\hline $\begin{array}{l}\text { EPA } \\
\text { Qual }\end{array}$ & Confidence & Unit \\
\hline--- & - & $-\cdots$ \\
\hline u & & $\mathrm{ug} / \mathrm{L}$ \\
\hline u & & $\mathrm{ug} / \mathrm{L}$ \\
\hline$u$ & & $\mathrm{ug} / \mathrm{L}$ \\
\hline u & & $\mathrm{ug} / \mathrm{L}$ \\
\hline u & & $u g / L$ \\
\hline u & & $\mathrm{ug} / \mathrm{L}$ \\
\hline u & & $\mathrm{ug} / \mathrm{L}$ \\
\hline u & & $\mathrm{ug} / \mathrm{L}$ \\
\hline u & & $\mathrm{ug} / \mathrm{L}$ \\
\hline$u$ & & $\mathrm{ug} / \mathrm{L}$ \\
\hline u & & $\mathrm{ug} / \mathrm{L}$ \\
\hline u & & $\mathrm{ug} / \mathrm{L}$ \\
\hline$u$ & & $u g / L$ \\
\hline u & & $\mathrm{ug} / \mathrm{L}$ \\
\hline u & & $\mathrm{ug} / \mathrm{L}$ \\
\hline$u$ & & $\mathrm{ug} / \mathrm{L}$ \\
\hline$u$ & & $\mathrm{ug} / \mathrm{L}$ \\
\hline$u$ & & $\mathrm{ug} / \mathrm{L}$ \\
\hline u & & $u g / L$ \\
\hline u & & $\mathrm{ug} / \mathrm{L}$ \\
\hline$u$ & & $\mathrm{ug} / \mathrm{L}$ \\
\hline$u$ & & $\mathrm{ug} / \mathrm{L}$ \\
\hline u & & $\mathrm{ug} / \mathrm{L}$ \\
\hline$u$ & & $u g / L$ \\
\hline u & & $\mathrm{ug} / \mathrm{L}$ \\
\hline u & & $\mathrm{ug} / \mathrm{L}$ \\
\hline$u$ & & $\mathrm{ug} / \mathrm{L}$ \\
\hline U & & $\mathrm{ug} / \mathrm{L}$ \\
\hline u & & $\mathrm{ug} / \mathrm{L}$ \\
\hline u & & $\mathrm{ug} / \mathrm{L}$ \\
\hline u & & $\mathrm{ug} / \mathrm{L}$ \\
\hline U & & $\mathrm{ug} / \mathrm{L}$ \\
\hline U & & $\mathrm{ug} / \mathrm{L}$ \\
\hline U & & $\mathrm{ug} / \mathrm{L}$ \\
\hline u & & $u g / L$ \\
\hline
\end{tabular}

UNCLASSIFIED 


\section{UNCLASSIFIED}

LIMS 03.08 .048

05/18/2010 14:02

\section{8}

205992

191242

207089

65850

111911

111444

108601

117817

85687

218019

84742

117840

53703

132649

84662

131113

06440

118741

77474
67721

193395

78591

621647

62759

86306

91203

98953

87865

85018

108952

129000

Benzo (a)pyrene

Benzo (b) fluoranthene

Benzo(ghi)perylene

Benzo(k) fluorar

Bis (2-chloroethoxy)methane

Bis (2-chloroethy 1) ether

Bis (2-chloroisopropy1) ether

Bis (2-ethylhexy1) phthalate

Butylbenzylphthalate

Carbazole

chrysene

Di-n-butylphthalate

Di-n-octylphthalate

Dibenz $(a, h)$ anthracene

Dibenzofuran

Diethylphthalate

Dimethylphthalate

Fluoranthene

Fluorene

Hexachlorobenzene

Hexachlorocyclopentadiene

Hexachloroethane

Indeno $(1,2,3$-cd) pyrene

Isophorone

$\mathrm{N}-\mathrm{Nit}$ roso-di-n-propylamine

$\mathrm{N}-\mathrm{Nit}$ rosodimethylamine

$\mathrm{N}$-Nit rosodiphenyl amine

Naphthalene

Nitrobenzene

Pentachlorophenol

Phenanthrene

Phenol

Pyrene

TIC Id

000078933 2-Butanone (CAS) \$S Methyl ethyl ketone

unknown alcohol/alkoxy cpd

unknown

004161608 2-Pentanone, 4-hydroxy- \$S $\mathrm{CH} 3 \mathrm{CH}(\mathrm{OH}) \mathrm{CH} 2 \mathrm{C}$

unknown alcoho

unknown

unknown

unknown

unknown

unknown

unknown

unknown

unknown

unknown

unknown

unknown

unknown

unknown

unknown

unknown
Analytical Chemistry Organization (Quality Services)

Page 2 of 3

P.o. Box 2009 oak Ridge, TN 378318244 (865) 574-2284

Lab Smp 1 Id: A101230505 official Report

Cust Smp1 Id: Co-60 300 SRS

500

500

2500

500

500

500

500

500

500
500

500
500

500

500

500

500

500

500

500

500
500

500

500
500

500

500
500

500
1000

500

500

500

110

Fn

Retention

Time

Unit

min

min

min

$\min$
$\min$

$\min$

min

min

min

min

min

$\min$

$\min$

$\min$

$\min$

$\min$

$\min$

$\min$
$\min$

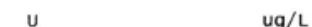

u ug/L

$u g / L$

$u g / L$

ug/L

$u g / L$

$\mathrm{ug} / \mathrm{L}$

$u g / L$

$u g / L$

ug/L

ug/L

ug/L

$\mathrm{ug} / \mathrm{L}$

$u g / L$

ug/L

$u g / L$

ug/L

$u g / L$

$u g / L$

ug/L

$u g / L$

ug/L

ug/L

$u g / L$

$\mathrm{ug} / \mathrm{L}$

ug/L

ug/L

ug/L

$u g / L$

ug/L

ug/L

ug/L

Estimated EPA

$\begin{array}{lll}1900 & --\cdot- & --- \\ -1 N & u g / L\end{array}$

$67000 \quad J$

17000

17000

2200
3200

1200

6500

2500

1300

2500

2200

2500

4300

45000

1500

50000

7600

26000
8900
Conc Qual Unit

Sample Test comments: Target compound(s) Initial calibration exceeding 15\% RSD:

$\begin{array}{ll}65-85-0 & \text { Benzoic acid } \\ 90-13-1 & 1-\text { Chloronaphthalene } \\ 51-28-5 & 2,4-D i n i t r o p h e n o 1 \\ 100-02-7 & 4-N i t r o p h e n o 1 \\ 92-87-5 & \text { Benzidine }\end{array}$

The mean Initial Calibration RSD $=10.6$

Comment objects:

Rpt Basis: AR

Test Req Cnt: 01

Analysis Method: SW846 9060 (Y50-AC-65-7314)

Approver: T J OATTS/Supervisor

QC Batch/File: QC10130012/10130A
Prep Method/Date:

Test Status: APPROVED

HT Deadline: 05/26/2010 23:59

Date Analyzed: 05/06/2010 08:30

Lab Group: IAWETC

UNCLASSIFIED 


\section{UNCLASSIFIED}

LIMS 03.08 .048

05/18/2010 14:02

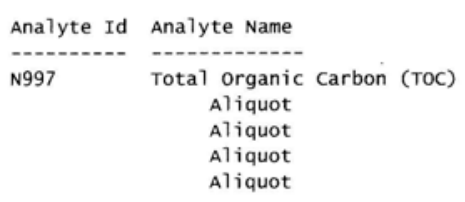

Comment objects:

EPA Qualifiers:

J - Estimated value.

N - Presumptive evidence of a compound. (GC/MS flag)

$U$ - Analyte analyzed for but undetected. Analyte result was below the contract required quantitation limit (CRQL).
Page 3 of 3 P.o. Box 2009 oak Ridge, TN 378318244 (865) 574-2284

Lab Smp1 Id: A101230505

$$
\text { official Report Cust Smpl Id: Co-60 } 300 \text { SRS }
$$

\begin{tabular}{lllllll}
\multicolumn{2}{c}{ Customer } & Dilution & Lower & & EPA & \\
HT Lim Fn & Factor & Limit & Result & Qual & Confidence & Unit \\
----- & -- & -200 & 1760 & & & $\mathrm{mg} / \mathrm{L}$ \\
& 200 & & 1711 & & & $\mathrm{mg} / \mathrm{L}$ \\
& 200 & & 1754 & & & $\mathrm{mg} / \mathrm{L}$ \\
& 200 & & 1782 & & & $\mathrm{mg} / \mathrm{L}$ \\
& 200 & & 1784 & & & $\mathrm{mg} / \mathrm{L}$ \\
& 200 & & & & &
\end{tabular}




\section{UNCLASSIFIED}

LIMS 03.08 .048

05/18/2010 14:00
Analytical Chemistry organization (Quality Services)

P.o. Box 2009 oak Ridge, TN 378318244 (865) 574-2284
Page 1 of 2

Lab Smp1 Id: A101230503

Project: L MS MPO SCHUH SVOA

Proj Mgr: L P BURNETT (865) 576-9141

Customer: L P BURNETT Bldg 9995 MS-8189 (865) 576-9141

\begin{tabular}{|c|c|c|c|c|c|c|c|}
\hline Matrix & $\begin{array}{l}\text { Chain of } \\
\text { Custody }\end{array}$ & $\begin{array}{l}\text { Work Agreement } \\
\text { Number }\end{array}$ & $\begin{array}{l}\text { Charge } \\
\text { Number }\end{array}$ & $\begin{array}{l}\text { Date } \\
\text { Sampled }\end{array}$ & $\begin{array}{l}\text { Date } \\
\text { Received }\end{array}$ & $\begin{array}{l}\text { Date } \\
\text { Needed }\end{array}$ & $\begin{array}{l}\text { Date } \\
\text { Approved }\end{array}$ \\
\hline----- & ------ & - n- & ---- & ---- & - - - & n- & - \\
\hline LIQUID & 117947 & 81473 & $745 A 0221$ & $04 / 28 / 2010 \quad 12: 30$ & $05 / 03 / 2010 \quad 15: 19$ & $05 / 17 / 2010 \quad 23: 59$ & $05 / 18 / 2010 \quad 14: 00$ \\
\hline
\end{tabular}

Sample Description:

Location:

Sample Status: APPROVED

Sampler(s):

Sample Approver: J T HOFFARTH/Data Approver

Customer comments:

Lab comments:

Comment objects:

$\ll<<$ Inorganic $\gg \gg>$

Rpt Basis: AR

Test Req Cnt: 01

Analysis Method: EPA 415.11983 (Y50-AC-65-7314)

Approver:

QC Batch/File:
Prep Method/Date:

Test Status: CANCELLED

HT Deadline: 05/26/2010 23:59

Date Analyzed:

Lab Group: IAWETC

Sample Test comments:

Change to TOC 9060 [027645 05/06/2010 06:14:23]

$\ll<<$ organic $\gg \gg>>$

Test: SVOA8270 (Semi-volatile organics by GC/MS)

Rpt Basis: AR Test Req Cnt: 01

Analysis Method: SW846 8270 C (Y50-AC-65-7316)

Approver: J T HOFFARTH/Data Approver

QC Batch/File: QC10138016/5385/53858
Prep Method/Date: Sw846 3580A 05/05/2010 15:00 Test Status: APPROVED

HT Deadline: 06/14/2010 23:59 Date Analyzed: 05/13/2010 12:18 Lab Group: OYGCMS

\begin{tabular}{|c|c|}
\hline Analyte Id & Analyte Name \\
\hline 120821 & (1)-Trichlerohenzene \\
\hline $\begin{array}{l}120821 \\
95501\end{array}$ & $\begin{array}{l}\text { 1,2,4-Trichlorobenzene } \\
\text { 1,2-Dichlorobenzene }\end{array}$ \\
\hline 122667 & 1,2-Diphenylhydrazine \\
\hline 541731 & 1,3-Dichlorobenzene \\
\hline 106467 & 1,4-Dichlorobenzene \\
\hline 95954 & 2,4,5-Trichlorophenol \\
\hline 88062 & 2,4,6-Trichlorophenol \\
\hline 120832 & 2,4-Dich1orophenol \\
\hline 105679 & 2,4-Dimethylphenol \\
\hline 51285 & 2,4-Dinitrophenol \\
\hline 121142 & 2,4-Dinitrotoluene \\
\hline 606202 & 2,6-Dinitrotoluene \\
\hline 91587 & 2-Chloronaphthalene \\
\hline 95578 & 2-Chlorophenol \\
\hline 534521 & 2-Methyl-4,6-dinitrophenol \\
\hline 91576 & 2-Methylnaphthalene \\
\hline 95487 & 2-Methylphenol \\
\hline 88744 & 2-Nitrobenzenamine \\
\hline 88755 & 2-Nitrophenol \\
\hline 91941 & 3,3'-Dichlorobenzidine \\
\hline N2799 & 3- and 4- Methylphenol \\
\hline 99092 & 3-Nitrobenzenamine \\
\hline 101553 & 4-Bromopheny 1 pheny 1 ether \\
\hline 59507 & 4-Chloro-3-methylphenol \\
\hline 106478 & 4-Chlorobenzenamine \\
\hline 7005723 & 4-Ch 1orophenylphenyl ether \\
\hline 100016 & 4-Nitrobenzenamine \\
\hline 100027 & 4-Nitrophenol \\
\hline 83329 & Acenaphthene \\
\hline 208968 & Acenaphthylene \\
\hline 62533 & Aniline \\
\hline 120127 & Anthracene \\
\hline 100516 & Benzenemethanol \\
\hline 92875 & Benzidine \\
\hline 56553 & Benzo (a) anthracene \\
\hline
\end{tabular}

\begin{tabular}{|c|c|c|c|c|c|c|c|c|}
\hline \multicolumn{3}{|c|}{ Customer } & \multirow{2}{*}{$\begin{array}{l}\text { Dilution } \\
\text { Factor }\end{array}$} & \multirow{2}{*}{$\begin{array}{l}\text { Lower } \\
\text { Limit }\end{array}$} & \multirow[b]{2}{*}{ Result } & \multirow{2}{*}{$\begin{array}{l}\text { EPA } \\
\text { Qual }\end{array}$} & \multirow[b]{2}{*}{ Confidence } & \multirow[b]{2}{*}{ Unit } \\
\hline HT & Lim & Fn & & & & & & \\
\hline-- & $\cdots$ & -- & & --.- & 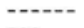 & 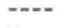 & - & $-\cdots$ \\
\hline & & & 1 & 99 & 500 & $u$ & & $\mathrm{ug} / \mathrm{L}$ \\
\hline & & & 1 & 110 & 500 & $u$ & & $\mathrm{ug} / \mathrm{L}$ \\
\hline & & & 1 & 59 & 500 & u & & $\mathrm{ug} / \mathrm{L}$ \\
\hline & & & 1 & 170 & 500 & u & & $\mathrm{ug} / \mathrm{L}$ \\
\hline & & & 1 & 180 & 500 & u & & $u g / L$ \\
\hline & & & 1 & 190 & 500 & $u$ & & $\mathrm{ug} / \mathrm{L}$ \\
\hline & & & 1 & 100 & 500 & $u$ & & $\mathrm{ug} / \mathrm{L}$ \\
\hline & & & 1 & 98 & 500 & u & & $\mathrm{ug} / \mathrm{L}$ \\
\hline & & & 1 & 99 & 500 & u & & $\mathrm{ug} / \mathrm{L}$ \\
\hline & & & 1 & 140 & 2500 & u & & ug/L \\
\hline & & & 1 & 130 & 500 & u & & ug/L \\
\hline & & & 1 & 97 & 1000 & u & & $\mathrm{ug} / \mathrm{L}$ \\
\hline & & & 1 & 87 & 500 & u & & $u g / L$ \\
\hline & & & 1 & 100 & 500 & u & & $u g / L$ \\
\hline & & & 1 & 110 & 500 & u & & $u g / L$ \\
\hline & & & 1 & 66 & 500 & $u$ & & $u g / L$ \\
\hline & & & 1 & 98 & 500 & u & & ug/L \\
\hline & & & 1 & 110 & 500 & u & & $\mathrm{ug} / \mathrm{L}$ \\
\hline & & & 1 & 81 & 500 & u & & $\mathrm{ug} / \mathrm{L}$ \\
\hline & & & 1 & 49 & 1000 & u & & $\mathrm{ug} / \mathrm{L}$ \\
\hline & & & 1 & 120 & 1000 & u & & $u g / L$ \\
\hline & & & 1 & 220 & 500 & u & & $\mathrm{ug} / \mathrm{L}$ \\
\hline & & & 1 & 66 & 500 & u & & $u g / L$ \\
\hline & & & 1 & 55 & 500 & u & & $\mathrm{ug} / \mathrm{L}$ \\
\hline & & & 1 & 100 & 500 & u & & $\mathrm{ug} / \mathrm{L}$ \\
\hline & & & 1 & 67 & 500 & u & & $u g / L$ \\
\hline & & & 1 & 110 & 1000 & u & & $\mathrm{ug} / \mathrm{L}$ \\
\hline & & & 1 & 100 & 2500 & u & & ug/L \\
\hline & & & 1 & 91 & 500 & u & & $u g / L$ \\
\hline & & & 1 & 100 & 500 & u & & ug/L \\
\hline & & & 1 & 40 & 1000 & u & & $u g / L$ \\
\hline & & & 1 & 52 & 500 & u & & $\mathrm{ug} / \mathrm{L}$ \\
\hline & & & 1 & 61 & 500 & u & & $\mathrm{ug} / \mathrm{L}$ \\
\hline & & & 1 & 120 & 2500 & u & & $u g / L$ \\
\hline & & & 1 & 57 & 500 & u & & $\mathrm{ug} / \mathrm{L}$ \\
\hline
\end{tabular}

UNCLASSIFIED 


\section{UNCLASSIFIED}

LIMS 03.08.048

05/18/2010 14:00

$\begin{array}{ll}50328 & \text { Benzo(a)pyrene } \\ 205992 & \text { Benzo(b)fluoranthene } \\ 191242 & \text { Benzo(ghi)perylene } \\ 207089 & \text { Benzo(k)fluoranthene } \\ 65850 & \text { Benzoic acid } \\ 111911 & \text { Bis(2-chloroethoxy)methane } \\ 111444 & \text { Bis(2-chloroethyl) ether } \\ 108601 & \text { Bis(2-chloroisopropy1) ether } \\ 117817 & \text { Bis(2-ethylhexyl)phthalate } \\ 85687 & \text { Butylbenzylphthalate } \\ 86748 & \text { Carbazole } \\ 218019 & \text { Chrysene } \\ 84742 & \text { Di-n-butylphthalate } \\ 117840 & \text { Di-n-octylphthalate } \\ 53703 & \text { Dibenz(a,h)anthracene } \\ 132649 & \text { Dibenzofuran } \\ 84662 & \text { Diethylphthalate } \\ 131113 & \text { Dimethylphthalate } \\ 206440 & \text { Fluoranthene } \\ 86737 & \text { Fluorene } \\ 118741 & \text { Hexachlorobenzene } \\ 77474 & \text { Hexachlorocyclopentadiene } \\ 67721 & \text { Hexachloroethane } \\ 193395 & \text { Indeno(1,2,3-cd)pyrene } \\ 78591 & \text { Isophorone } \\ 621647 & \text { N-Nitroso-di-n-propylamine } \\ 62759 & \text { N-Nitrosodimethylamine } \\ 86306 & \text { N-Nitrosodiphenylamine } \\ 91203 & \text { Naphthalene } \\ 98953 & \text { Nitrobenzene } \\ 87865 & \text { Pentachlorophenol } \\ 85018 & \text { Phenanthrene } \\ 108952 & \text { Phenol } \\ 129000 & \text { Pyrene } \\ & \\ \text { TIC Id } & \text { TIC Name } \\ ---- & \text {------ } \\ 000123422 & \begin{array}{l}\text { unknown } \\ \text { 2-Pentanone, }\end{array} \\ & \end{array}$

000123422 2-Pentanone, 4-hydroxy-4-methyl-(CAS) \$
Analytical Chemistry organization (Quality Services)

Page 2 of 2

P.0. Box 2009 Oak Ridge, TN 378318244 (865) 574-2284

Lab Smp1 Id: A101230503
Cust Smp1 Id: Co-60 300 water

$500 \quad u \quad u g / L$

$500 \quad$ ug/L

$500 \quad u \quad u g / L$

$500 \quad$ U ug/L

$\begin{array}{lll}2500 & \text { U } & \text { ug/L } \\ 500 & \text { U } & \text { ug/L }\end{array}$

$500 \quad u$

$500 \quad$ U ug/L

$500 \quad u \quad u g / L$

$500 \quad u \quad u g / L$

$\begin{array}{lll}500 & U & \mathrm{ug} / \mathrm{L} \\ 500 & \mathrm{u} & \mathrm{ug} / \mathrm{L}\end{array}$

500

$500 \quad u \quad u g / L$

$500 \quad u \quad u g / L$

$500 \quad u \quad$ ug/L

$500 \quad u \quad u g / L$

$500 \quad$ U $\quad$ ug/L

500

500

500

500

500

500

500
500

500

500

500

1000
500

500

ug/L

$\mathrm{ug} / \mathrm{L}$

ug/L

$u g / L$

$u g / L$

$\mathrm{ug} / \mathrm{L}$

$u g / L$

ug/L

$u g / \mathrm{L}$

ug/L

$\mathrm{ug} / \mathrm{L}$

$\mathrm{ug} / \mathrm{L}$

ug/L

$\mathrm{ug} / \mathrm{L}$

$\mathrm{ug} / \mathrm{L}$

110

500

Estimated EPA

Conc Qua?

Unit

min

min

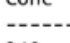

240

330

Sample Test Comments: Target Compound(s) Initial Calibration exceeding 15\% RSD:

$\begin{array}{ll}65-85-0 & \text { Benzoic acid } \\ 90-13-1 & 1-\text { Chloronaphthalene } \\ 51-28-5 & 2,4-\text { Dinitrophenol } \\ 100-02-7 & 4-N i t r o p h e n o l \\ 92-87-5 & \text { Benzidine }\end{array}$

The mean Initial calibration RSD $=1$

Comment objects:

Test: Toc9060 (Total Organic Carbon)

Rpt Basis: AR Test Req Cnt: 01

Analysis Method: Sw846 9060 (Y50-AC-65-7314)

Approver: T J OATTS/Supervisor

QC Batch/File: QC10130012/10130A

Prep Method/Date:

Test Status: APPROVED

HT Deadline: 05/26/2010 23:59

Date Analyzed: 05/06/2010 08:30

Lab Group: IAWETC

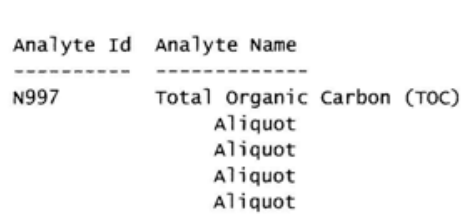

\begin{tabular}{lll}
\multicolumn{2}{c}{ Customer } & Dilution \\
HT Lim Fn & Factor \\
----- & -- & $-\cdot 20$ \\
& & 20 \\
& & 20 \\
& & 20 \\
& &
\end{tabular}
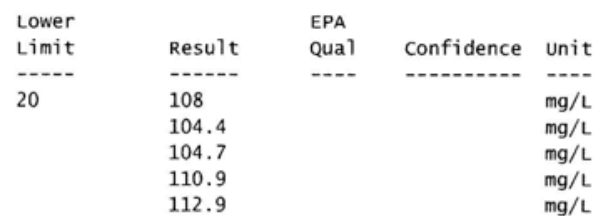

Comment objects:

EPA Qualifiers:

J - Estimated value.

N - Presumptive evidence of a compound. (GC/MS flag)

$U$ - Analyte analyzed for but undetected. Analyte result was below the contract required quantitation limit (CRQL).

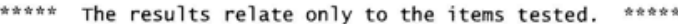

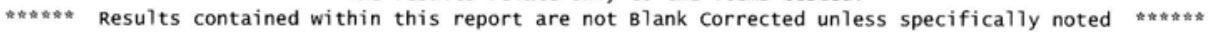

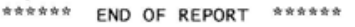

UNCLASSIFIED 


\section{UNCLASSIFIED}

LIMS 03.08 .048

05/18/2010 14:01
Analytical Chemistry organization (Quality Services)

P.o. Box 2009 oak Ridge, TN 378318244 (865) 574-2284
Page 1 of 2

Lab Smp1 Id: A101230504

Project: L MS MPO SCHUH SVOA

Proj Mgr: L P BURNETT (865) 576-9141

Customer: L P BURNETT B Bldg 9995 MS-8189 (865) 576-9141

\begin{tabular}{|c|c|c|c|c|c|c|c|}
\hline Matrix & $\begin{array}{l}\text { Chain of } \\
\text { Custody }\end{array}$ & $\begin{array}{l}\text { Work Agreement } \\
\text { Number }\end{array}$ & $\begin{array}{l}\text { Charge } \\
\text { Number }\end{array}$ & $\begin{array}{l}\text { Date } \\
\text { Sampled }\end{array}$ & $\begin{array}{l}\text { Date } \\
\text { Received }\end{array}$ & $\begin{array}{l}\text { Date } \\
\text { Needed }\end{array}$ & $\begin{array}{l}\text { Date } \\
\text { Approved }\end{array}$ \\
\hline --n- & ------ & $\cdots$ & 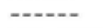 & -....- & -n.-. & $\cdots-$ & - - \\
\hline LIQUID & 117947 & 81473 & $745 A 0221$ & $04 / 28 / 2010 \quad 12: 30$ & $05 / 03 / 2010 \quad 15: 19$ & $05 / 17 / 2010 \quad 23: 59$ & $05 / 18 / 2010$ \\
\hline
\end{tabular}

Sample Description:

Location:

Sample Status: APPROVED

Sampler(s):

Sample Approver: J T HOFFARTH/Data Approver

Customer comments:

Lab Comments:

Comment objects:

$\ll<<$ Inorganic $\gg \gg>$

Test: TOC (Total Organic Carbon, 415.1)

Rpt Basis: AR

Test Req Cnt: 01

Analysis Method: EPA 415.11983 (Y50-AC-65-7314)

Approver:

QC Batch/File:

Prep Method/Date:

Test Status: CANCELLED

HT Deadline: 05/26/2010 23:59

Date Analyzed:

Lab Group: IAWETC

Sample Test comments:

Change to TOC $9060[027645 \quad 05 / 06 / 2010 \quad 06: 14: 46]$

$\ll<<$ Organic $\gg \gg>$

Test: SVOA8270 (Semi-volatile organics by GC/MS)

Rpt Basis: AR Test Req Cnt: 01

Analysis Method: SW846 $8270 \mathrm{C}$ (Y50-AC-65-7316)

Approver: J T HOFFARTH/Data Approver

QC Batch/File: QC10138016/5385/53858
Prep Method/Date: SW846 3580A 05/05/2010 15:00 Test Status: APPROVED

HT Deadline: 06/14/2010 23:59

Date Analyzed: 05/13/2010 13:05

Lab Group: OYGCMS

\begin{tabular}{ll} 
Analyte Id & Analyte Name \\
\hline 120821 & 1,2,4-Trichlorobenzene \\
95501 & 1,2-Dichlorobenzene \\
122667 & 1,2-Diphenylhydrazine \\
541731 & 1,3-Dichlorobenzene \\
106467 & 1,4-Dichlorobenzene \\
95954 & 2,4,5-Trichlorophenol \\
88062 & 2,4,6-Trichlorophenol \\
120832 & 2,4-Dichlorophenol \\
105679 & 2,4-Dimethylpheno1 \\
51285 & 2,4-Dinitropheno1 \\
121142 & 2,4-Dinitrotoluene \\
606202 & 2,6-Dinitrotoluene \\
91587 & 2-Chloronaphthalene \\
95578 & 2-Chlorophenol \\
534521 & 2-Methyl-4,6-dinitropheno1 \\
91576 & 2-Methylnaphthalene \\
95487 & 2-Methylphenol \\
88744 & 2-Nitrobenzenamine \\
88755 & 2-Nitrophenol \\
91941 & 3,3'-Dichlorobenzidine \\
N2799 & 3- and 4- Methylpheno1 \\
99092 & 3-Nitrobenzenamine \\
101553 & 4-Bromophenyl phenyl ether \\
59507 & 4-Chloro-3-methylpheno1 \\
106478 & 4-Chlorobenzenamine \\
7005723 & 4-Chlorophenylphenyl ether \\
100016 & 4-Nitrobenzenamine \\
100027 & 4-Nitrophenol \\
83329 & Acenaphthene \\
208968 & Acenaphthylene \\
62533 & Aniline \\
120127 & Anthracene \\
100516 & Benzenemethanol \\
92875 & Benzidine \\
56553 & Benzo(a)anthracene \\
& \\
\hline 553
\end{tabular}

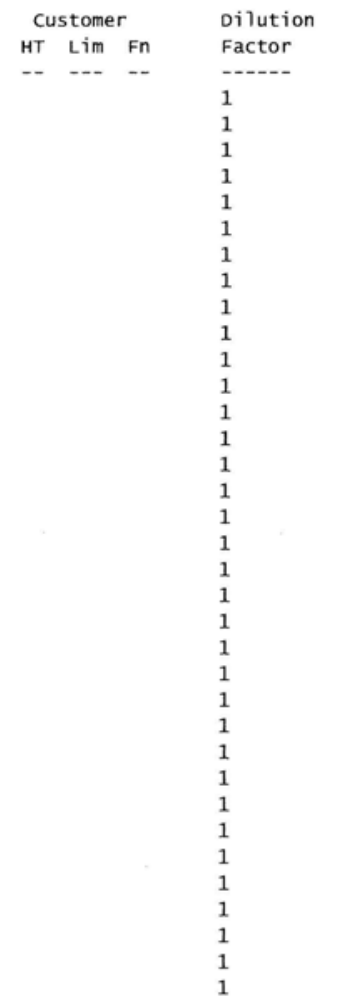

\begin{tabular}{|c|c|c|c|}
\hline Result & $\begin{array}{l}\text { EPA } \\
\text { Qual }\end{array}$ & Confidence & Unit \\
\hline - & -- & - & -- \\
\hline 500 & $u$ & & ug/L \\
\hline 500 & $u$ & & $u g / L$ \\
\hline 500 & $u$ & & $u g / L$ \\
\hline 500 & $u$ & & $u g / L$ \\
\hline 500 & u & & $u g / L$ \\
\hline 500 & u & & $\mathrm{ug} / \mathrm{L}$ \\
\hline 500 & $u$ & & $u g / L$ \\
\hline 500 & u & & $u g / L$ \\
\hline 500 & u & & $u g / L$ \\
\hline 2500 & u & & $\mathrm{ug} / \mathrm{L}$ \\
\hline 500 & u & & $\mathrm{ug} / \mathrm{L}$ \\
\hline 1000 & U & & $\mathrm{ug} / \mathrm{L}$ \\
\hline 500 & $u$ & & $\mathrm{ug} / \mathrm{L}$ \\
\hline 500 & u & & $\mathrm{ug} / \mathrm{L}$ \\
\hline 500 & u & & $\mathrm{ug} / \mathrm{L}$ \\
\hline 500 & u & & $\mathrm{ug} / \mathrm{L}$ \\
\hline 500 & u & & $\mathrm{ug} / \mathrm{L}$ \\
\hline 500 & u & & ug/L \\
\hline 500 & u & & $\mathrm{ug} / \mathrm{L}$ \\
\hline 1000 & u & & $\mathrm{ug} / \mathrm{L}$ \\
\hline 1000 & u & & $\mathrm{ug} / \mathrm{L}$ \\
\hline 500 & u & & $\mathrm{ug} / \mathrm{L}$ \\
\hline 500 & U & & $\mathrm{ug} / \mathrm{L}$ \\
\hline 500 & u & & $u g / L$ \\
\hline 500 & u & & $\mathrm{ug} / \mathrm{L}$ \\
\hline 500 & u & & $\mathrm{ug} / \mathrm{L}$ \\
\hline 1000 & u & & $\mathrm{ug} / \mathrm{L}$ \\
\hline 2500 & u & & $\mathrm{ug} / \mathrm{L}$ \\
\hline 500 & u & & $u g / L$ \\
\hline 500 & U & & $\mathrm{ug} / \mathrm{L}$ \\
\hline 1000 & U & & $\mathrm{ug} / \mathrm{L}$ \\
\hline 500 & u & & ug/L \\
\hline 500 & $\mathrm{u}$ & & ug/L \\
\hline 2500 & u & & $\mathrm{ug} / \mathrm{L}$ \\
\hline & 0 & & $u g / L$ \\
\hline
\end{tabular}

UNCLASSIFIED 
LIMS 03.08 .048

05/18/2010 14:01

$\begin{array}{ll}50328 & \text { Benzo(a)pyrene } \\ 205992 & \text { Benzo(b)fluoranthene } \\ 191242 & \text { Benzo(ghi)perylene } \\ 207089 & \text { Benzo(k)fluoranthene } \\ 65850 & \text { Benzoic acid } \\ 111911 & \text { Bis(2-chloroethoxy)methane } \\ 111444 & \text { Bis(2-chloroethyl) ether } \\ 108601 & \text { Bis(2-chloroisopropyl) ether } \\ 117817 & \text { Bis(2-ethylhexyl)phthalate } \\ 85687 & \text { Butylbenzylphthalate } \\ 86748 & \text { Carbazole } \\ 218019 & \text { Chrysene } \\ 84742 & \text { Di-n-butylphthalate } \\ 117840 & \text { Di-n-octylphthalate } \\ 53703 & \text { Dibenz(a,h)anthracene } \\ 132649 & \text { Dibenzofuran } \\ 84662 & \text { Diethylphthatate } \\ 131113 & \text { Dimethylphthalate } \\ 206440 & \text { Fluoranthene } \\ 86737 & \text { Fluorene } \\ 118741 & \text { Hexachlorobenzene } \\ 77474 & \text { Hexachlorocyclopentadiene } \\ 67721 & \text { Hexachloroethane } \\ 193395 & \text { Indeno(1,2,3-cd)pyrene } \\ 78591 & \text { Isophorone } \\ 621647 & \text { N-Nitroso-di-n-propylamine } \\ 62759 & \text { N-Nitrosodimethylamine } \\ 86306 & \text { N-Nitrosodiphenylamine } \\ 91203 & \text { Naphthalene } \\ 98953 & \text { Nitrobenzene } \\ 87865 & \text { Pentachlorophenol } \\ 85018 & \text { Phenanthrene } \\ 108952 & \text { Phenol } \\ 129000 & \text { Pyrene } \\ & \\ T I C \text { Id } & \text { TIC Name } \\ ---- & \text {------ } \\ & \text { unknown } \\ 000123422 & \text { 2-Pentanone, } 4-h y d r o x y-4-n \\ & \end{array}$

000123422 2-Pentanone, 4-hydroxy-4-methy1- (CAS) \$

\section{UNCLASSIFIED}

Analytical Chemistry organization (Quality Services)

Page 2 of 2

P.o. Box 2009 oak Ridge, TN 378318244 (865) 574-2284

Lab Smp1 Id: A101230504

official Report

Cust Smpl Id: Co-60 300 nitric

\begin{tabular}{|c|c|c|c|c|c|c|}
\hline 1 & 45 & & 500 & u & & $u g / L$ \\
\hline 1 & 110 & & 500 & u & & $u g / L$ \\
\hline 1 & 150 & & 500 & u & & $u g / L$ \\
\hline 1 & 150 & & 500 & u & & $u g / L$ \\
\hline 1 & 190 & & 2500 & u & & $\mathrm{ug} / \mathrm{L}$ \\
\hline 1 & 53 & & 500 & u & & $\mathrm{ug} / \mathrm{L}$ \\
\hline 1 & 140 & & 500 & u & & ug/L \\
\hline 1 & 70 & & 500 & u & & $\mathrm{ug} / \mathrm{L}$ \\
\hline 1 & 120 & & 500 & u & & $\mathrm{ug} / \mathrm{L}$ \\
\hline 1 & 120 & & 500 & u & & $\mathrm{ug} / \mathrm{L}$ \\
\hline 1 & 61 & & 500 & u & & $\mathrm{ug} / \mathrm{L}$ \\
\hline 1 & 97 & . & 500 & $u$ & & ug/L \\
\hline 1 & 69 & & 500 & u & & $\mathrm{ug} / \mathrm{L}$ \\
\hline 1 & 140 & & 500 & u & & $\mathrm{ug} / \mathrm{L}$ \\
\hline 1 & 160 & & 500 & u & & $\mathrm{ug} / \mathrm{L}$ \\
\hline 1 & 92 & & 500 & u & & $u g / L$ \\
\hline 1 & 87 & & 500 & u & & $\mathrm{ug} / \mathrm{L}$ \\
\hline 1 & 100 & & 500 & u & & $\mathrm{ug} / \mathrm{L}$ \\
\hline 1 & 51 & & 500 & u & & $\mathrm{ug} / \mathrm{L}$ \\
\hline 1 & 74 & & 500 & u & & ug/L \\
\hline 1 & 66 & & 500 & u & & $\mathrm{ug} / \mathrm{L}$ \\
\hline 1 & 260 & & 500 & u & & ug/L \\
\hline 1 & 150 & & 500 & u & & $\mathrm{ug} / \mathrm{L}$ \\
\hline 1 & 100 & & 500 & u & & $\mathrm{ug} / \mathrm{L}$ \\
\hline 1 & 70 & & 500 & U & & $\mathrm{ug} / \mathrm{L}$ \\
\hline 1 & 65 & & 500 & U & & $\mathrm{ug} / \mathrm{L}$ \\
\hline 1 & 150 & & 500 & u & & ug/L \\
\hline 1 & 59 & & 500 & u & & $\mathrm{ug} / \mathrm{L}$ \\
\hline 1 & 97 & & 500 & $u$ & & $u g / L$ \\
\hline 1 & 48 & & 500 & u & & $\mathrm{ug} / \mathrm{L}$ \\
\hline 1 & 54 & & 1000 & u & & $\mathrm{ug} / \mathrm{L}$ \\
\hline 1 & 62 & & 500 & u & & $\mathrm{ug} / \mathrm{L}$ \\
\hline 1 & 92 & & 500 & u & & $\mathrm{ug} / \mathrm{L}$ \\
\hline \multirow[t]{6}{*}{1} & 110 & & 500 & $u$ & & $u g / L$ \\
\hline & \multicolumn{3}{|c|}{ Retention } & Estimated & EPA & \\
\hline & Time & & Unit & Conc & Qua 1 & Unit \\
\hline & --- & & --- & - & 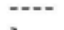 & --- \\
\hline & 6.42 & & $\min$ & 640 & J & $\mathrm{ug} / \mathrm{L}$ \\
\hline & 7.29 & & $\min$ & 380 & JN & $\mathrm{ug} / \mathrm{L}$ \\
\hline
\end{tabular}

Sample Test Comments: Target Compound(s) Initial Calibration exceeding 15\% RSD:

$\begin{array}{ll}65-85-0 & \text { Benzoic acid } \\ 90-13-1 & 1-\text { Chloronaphthalene } \\ 51-28-5 & 2,4-\text { Dinitrophenol } \\ 100-02-7 & 4-\text { Nitrophenol } \\ 92-87-5 & \text { Benzidine } \\ \text { The mean Initial Calibration RSD }=10.6\end{array}$

Comment objects:

Test: Toc9060 (Total organic Carbon)

Rpt Basis: AR

Test Req Cnt: 01

Analysis Method: SW846 9060 (Y50-AC-65-7314)

Approver: T J OATTS/Supervisor

QC Batch/File: QC10130012/10130A

Prep Method/Date:

Test Status: APPROVED

HT Deadline: 05/26/2010 23:59

Date Analyzed: 05/06/2010 08:30

Lab Group: IAWETC
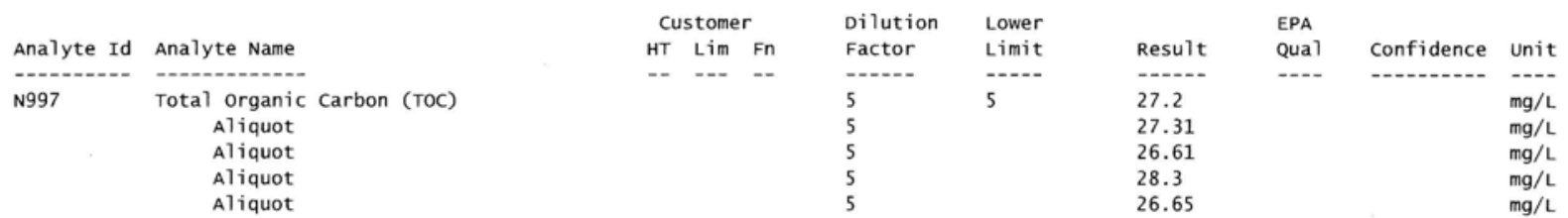

Comment objects:

EPA Qualifiers:

J - Estimated value.

N - Presumptive evidence of a compound. (GC/MS flag)

$U$ - Analyte analyzed for but undetected. Analyte result was below the contract required quantitation limit (CRQL).

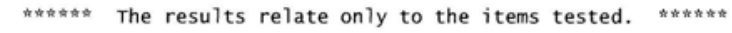

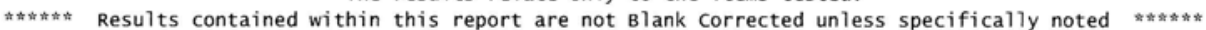

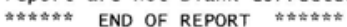

\section{UNCLASSIFIED}




\section{TestAmerica}

THE LEADER IN ENVIRONMENTAL TESTING

2960 Foster Creighton Road Nashville, TN 37204 * 800-765-0980 * Fax 615-726-3404

$\begin{array}{ll}\text { Client } & \text { Oak Ridge National Laboratory (415880) } \\ & \text { 1 Bethal Valley Road } \\ & \text { Oak Ridge, TN } 37931 \\ \text { Attn } \quad \text { Paul Allen Taylor }\end{array}$

Work Order: $\quad$ NTE1950

Project Name: $\quad$ SCIX

Project Number: [none]

Received:

05/20/10 08:00

\section{ANALYTICAL REPORT}

\begin{tabular}{|c|c|c|c|c|c|c|c|c|}
\hline Analyte & Result & Flag & Units & MRL & $\begin{array}{c}\text { Dilution } \\
\text { Factor }\end{array}$ & $\begin{array}{l}\text { Analysis } \\
\text { Date/Time }\end{array}$ & Method & Batch \\
\hline
\end{tabular}

Sample ID: NTE1950-01 (SRS Salt - Water) Sampled: 05/18/10 00:01

Aldehydes by EPA Method 8315A

Formaldehyde

ND $\quad$ ug/L

$11 \%$

/ $\quad 50.0$

05/23/10 19:07 SW846 8315A

$10 \mathrm{E} 3454$

Surr: Butyraldehyde (10-150\%)

05/23/10 19:07 SW8468315A

IOE 3454

Sample ID: NTE1950-02 (Water - Water) Sampled: 05/18/10 00:01

Aldehydes by EPA Method $8315 \mathrm{~A}$

Formaldehyde

61.4

$\mathrm{ug} / \mathrm{L}$

50.0

$101 \%$

Surr: Butyraldehyde (10-150\%)

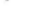

$\begin{array}{rrr}05 / 23 / 1019: 26 & \text { SW846 8315A } & \text { 10E3454 } \\ 05 / 23 / 10 ~ 19: 26 & \text { SW846 83I5A } & \text { IOE3454 }\end{array}$




\section{TestAmerica}

THE LEADER IN ENVIRONMENTAL TESTING

2960 Foster Creighton Road Nashville, TN $37204 * 800-765-0980 *$ Fax 615-726-3404

$\begin{array}{ll}\text { Client } & \text { Oak Ridge National Laboratory (415880) } \\ & \text { 1 Bethal Valley Road } \\ & \text { Oak Ridge, TN } 37931 \\ \text { Attn } \quad \text { Paul Allen Taylor }\end{array}$

Work Order: $\quad$ NTE1822

Project Name: $\quad$ SCIX

Project Number: [none]

Received: $\quad 05 / 19 / 1008: 00$

\section{ANALYTICAL REPORT}

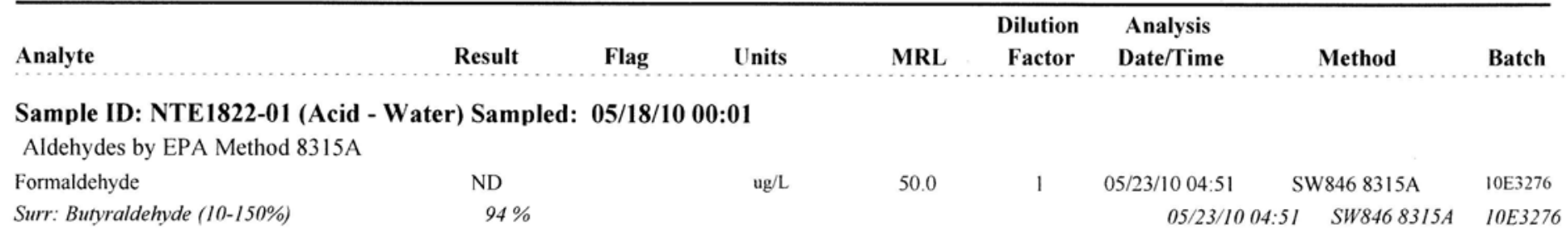


UNCLASSIFIED

LIMS 03.08.048

02/22/2010 07:01
Analytical Chemistry organization (Quality Services)

P.O. Box 2009 oak Ridge, TN 378318244 (865) 574-2284

official Report
Page 1 of 2

Lab Smp 1 Id: A100250073

Project: L MS MPO SCHUH SVOA

Proj Mgr: L P BURNETT (865) 576-9141

Cust Smp 1 Id: HFIR SRS $300 \mathrm{Mrad} 2 \mathrm{MNaOH}$

Customer: L. P BURNETT B 7 dg 9995 MS-8189 (865) 576-9141

\begin{tabular}{|c|c|c|c|c|c|c|c|}
\hline Matrix & $\begin{array}{l}\text { Chain of } \\
\text { Custody }\end{array}$ & $\begin{array}{l}\text { Work Agreement } \\
\text { Number }\end{array}$ & $\begin{array}{l}\text { Charge } \\
\text { Number }\end{array}$ & $\begin{array}{l}\text { Date } \\
\text { Sampled }\end{array}$ & $\begin{array}{l}\text { Date } \\
\text { Received }\end{array}$ & $\begin{array}{l}\text { Date } \\
\text { Needed }\end{array}$ & $\begin{array}{l}\text { Date } \\
\text { Approved }\end{array}$ \\
\hline$-\cdots-$ & - & ---- & ----- & - - & - n-n- & $\cdots+-$ & - \\
\hline LIQUID & 117929 & 81473 & $745 A 0221$ & $01 / 21 / 2010 \quad 00: 00$ & $01 / 21 / 2010 \quad 14: 30$ & $02 / 04 / 2010 \quad 23: 59$ & $02 / 22 / 2010 \quad 07: 01$ \\
\hline
\end{tabular}

Sample Description:

Sample Status: APPROVED

Sample Approver: J T HOFFARTH/Data Approver

Customer comments:

Lab Comments:

Comment objects:

$\ll<$ Organic $\gg \gg>$

Test: SVOA8270 (Semi-Volatile Organics by GC/MS)

Rpt Basis: AR Test Req Cnt: 01

Analysis Method: SW846 8270C (Y50-AC-65-7316)

Approver: J T HOFFARTH/Data Approver

QC Batch/File: QC10049010/5281/53758
Prep Method/Date: SW846 3580A 02/01/2010 13:00 Test Status: APPROVED

HT Deadline: 03/13/2010 23:59

Date Analyzed: 02/15/2010 17:24 Lab Group: oYGCMS

\begin{tabular}{|c|c|}
\hline Analyte Id & Analyte Name \\
\hline 120821 & - \\
\hline $\begin{array}{l}120821 \\
95501\end{array}$ & 1,2,4-Trichlorobenzene \\
\hline 95501 & 1,2-Dichlorobenzene \\
\hline 122667 & 1,2-Diphenylhydrazine \\
\hline 541731 & 1,3-Dichlorobenzene \\
\hline 106467 & 1,4-Dichlorobenzene \\
\hline 95954 & 2,4,5-Trichlorophenol \\
\hline 88062 & 2,4,6-Trichlorophenol \\
\hline 120832 & 2,4-Dichlorophenol \\
\hline 105679 & 2,4-Dimethylphenol \\
\hline 51285 & 2,4-Dinitrophenol \\
\hline 121142 & 2,4-Dinitrotoluene \\
\hline 606202 & 2,6-Dinitrotoluene \\
\hline 91587 & 2-Chloronaphthalene \\
\hline 95578 & 2-Chlorophenol \\
\hline 534521 & 2-Methy $1-4,6$-dinitrophenol \\
\hline 91576 & 2-Methylnaphthalene \\
\hline 95487 & 2-Methylphenol \\
\hline 88744 & 2-Nitrobenzenamine \\
\hline 88755 & 2-Nitrophenol \\
\hline 91941 & 3, 3'-Dichlorobenzidine \\
\hline N2799 & 3- and 4- Methylphenol \\
\hline 99092 & 3-Nitrobenzenamine \\
\hline 101553 & 4-Bromophenyl phenyl ether \\
\hline 59507 & 4-Chloro-3-methylphenol \\
\hline 106478 & 4-Ch1orobenzenamine \\
\hline 7005723 & 4-Chloropheny 1 pheny 1 ether \\
\hline 100016 & 4-Nitrobenzenamine \\
\hline 100027 & 4-Nit rophenol \\
\hline 83329 & Acenaphthene \\
\hline 208968 & Acenaphthylene \\
\hline 62533 & Aniline \\
\hline 120127 & Anthracene \\
\hline 100516 & Benzenemethanol \\
\hline 92875 & Benzidine \\
\hline 56553 & Benzo(a)anthracene \\
\hline 50328 & Benzo(a)pyrene \\
\hline 205992 & Benzo(b) fluoranthene \\
\hline 191242 & Benzo(ghi)perylene \\
\hline 207089 & Benzo $(k)$ fluoranthene \\
\hline 65850 & Benzoic acid \\
\hline 111911 & Bis (2-chloroethoxy)methane \\
\hline 111444 & Bis(2-chloroethy1) ether \\
\hline 108601 & Bis (2-chloroisopropyl) ether \\
\hline 117817 & Bis(2-ethylhexy1)phthalate \\
\hline 85687 & Butylbenzylphthalate \\
\hline 86748 & Carbazole \\
\hline 218019 & Chrysene \\
\hline 84742 & Di-n-butylphthalate \\
\hline 117840 & Di-n-octylphthalate \\
\hline 53703 & Dibenz $(\mathrm{a}, \mathrm{h})$ anthracene \\
\hline
\end{tabular}

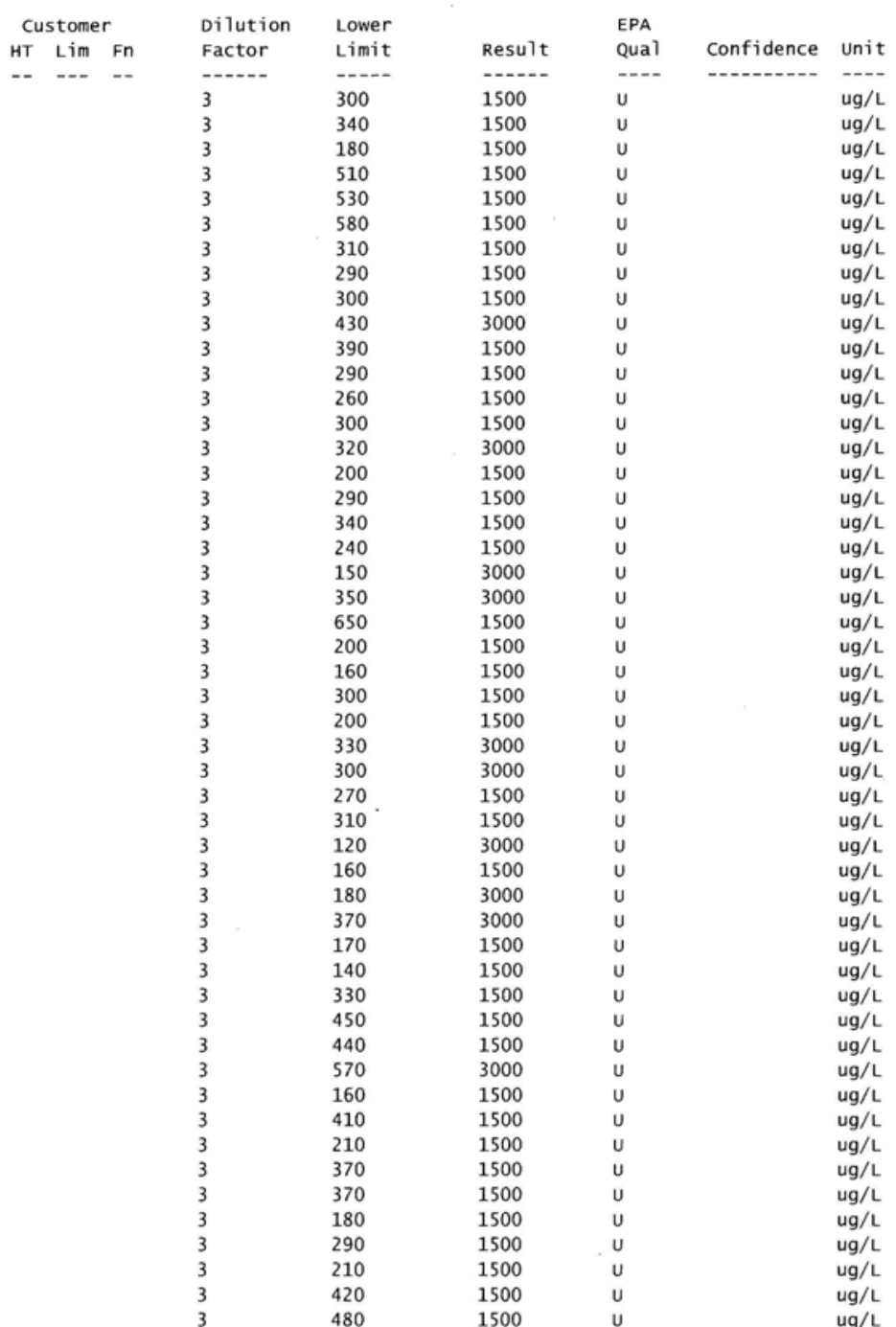

UNCLASSIFIED 
UNCLASSIFIED

LIMS 03.08.048

02/22/2010 07:01

132649 Dibenzofuran

84662 Diethylphthalate

131113 Dimethylphthalate

$206440 \quad$ Fluoranthene

86737 Fluorene

118741 Hexachlorobenzene

77474 Hexachlorocyclopentadiene

67721 Hexachloroethane

193395 Indeno(1,2,3-cd)pyrene

78591 Isophorone

$621647 \quad \mathrm{~N}$-Nitroso-di-n-propylamine

$62759 \quad \mathrm{~N}$-Nitrosodimethylamine

$86306 \quad \mathrm{~N}$-Nitrosodiphenylamine

91203 Naphthalene

$98953 \quad$ Nitrobenzene

$87865 \quad$ Pentachlorophenol

$85018 \quad$ Phenanthrene

108952 Phenol

129000 Pyrene

\section{TIC Id TIC Name}

000000000 2,4-DINITROPHENOL-D3

$000000000 \quad 2$-NITROPHENOL-D4

unknown

001794861 Phosgene oxime \$\$ Phosgenox \$\$ CX \$\$ Hyd

007119893 Methane, dichloronitro- \$\$ Dichloronitro Diacetone Alcohol

002562370 Cyclohexene, 1-nitro- \$\$ 1-Nitrocyclohex

007459714 3,5-Dimethylcyclopentene \$\$ 3,5-Dimethyl

043080235 2-Propenoic acid, pentadecyl ester \$\$ 2-

000999780 2-Pentyne, 4,4-dimethy1- \$\$ 4,4-Dimethy?

unknown

unknown

unknown

unknown

unknown

unknown

unknown

unknown

unknown

unknown
Analytical Chemistry organization (Quality Services) P.O. Box 2009 oak Ridge, TN 378318244 (865) 574-2284

Page 2 of 2 official Report

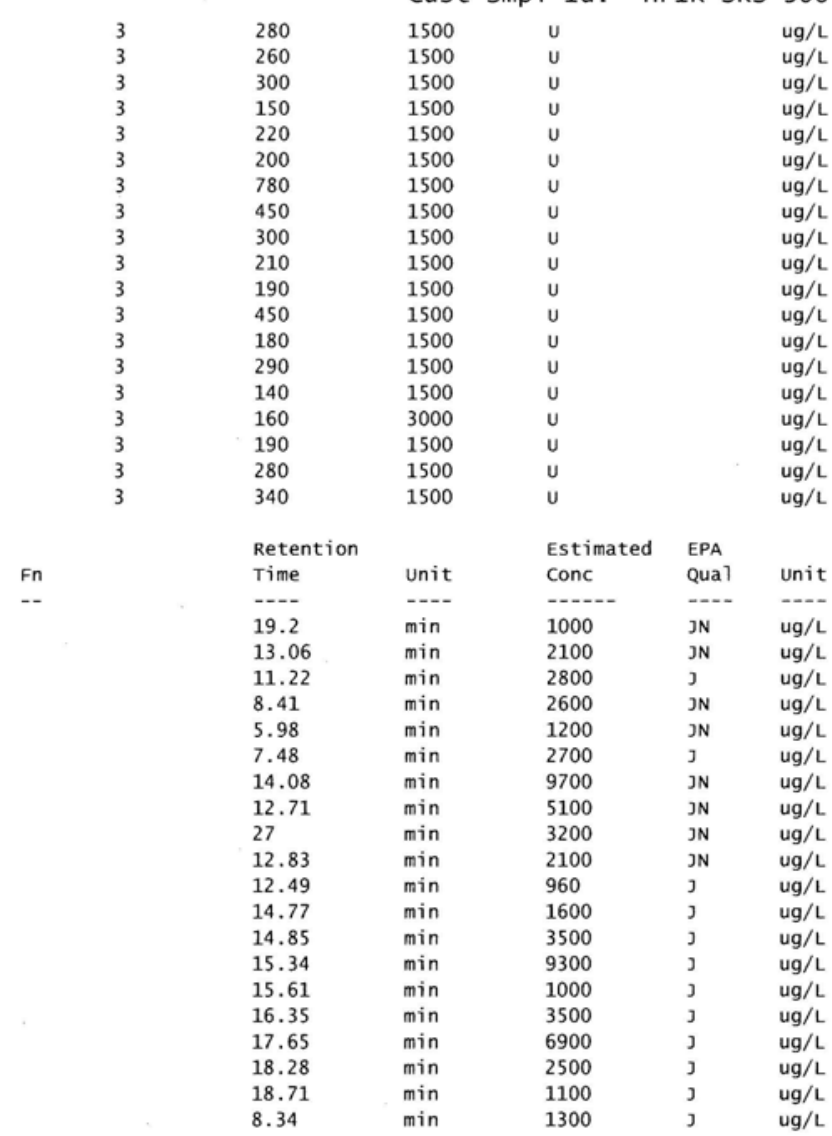

Sample Test Comments: Target compound(s) Initial Calibration exceeding 15\% RSD: 62-53-3 Aniline 207-08-9 Benzo[k]fluoranthene

The mean Initial Calibration $\mathrm{RSD}=8.7$

Comment objects:

EPA Qualifiers:

J - Estimated value.

$\mathrm{N}$ - Presumptive evidence of a compound. (GC/MS flag)

$U$ - Analyte analyzed for but undetected. Analyte result was below the contract required quantitation limit (CRQL).

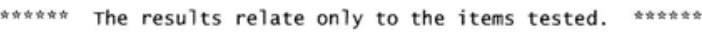

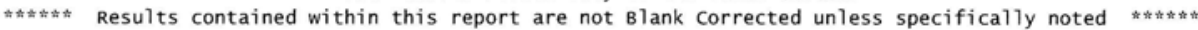

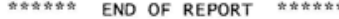

UNCLASSIFIED 
UNCLASSIFIED

LIMS 03.08.048

Analytical Chemistry organization (Quality Services)

Page 1 of 2

02/22/2010 07:03

P.o. Box 2009 oak Ridge, TN 378318244 (865) 574-2284

Lab Smp1 Id: A100250075

official Report

Cust Smpl Id: HFIR SRS $250 \mathrm{Mräd} 2 \mathrm{MNaOH}$

Project: L MS MPO SCHUH SVOA

Proj Mgr: L P BURNETT (865) 576-9141

Customer: L P BURNETT B1dg 9995 MS-8189 (865) 576-9141

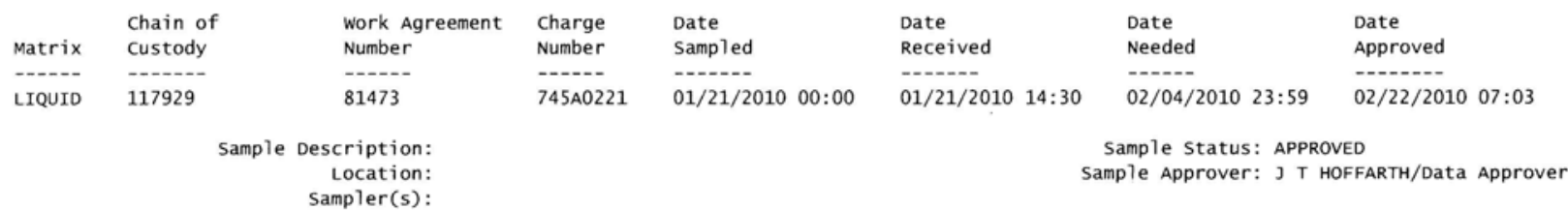

Customer comments:

Lab comments:

Comment objects:

$\ll<<$ Organic $\gg \gg>>$

Test: SVOA8270 (Semi-volatile organics by GC/MS)

Analysis Method: SW846 8270C (Y50-AC-65-7316)

Approver: ] T HOFFARTH/Data Approver

QC Batch/File: QC10049010/5281/53758

Prep Method/Date: SW846 3580A 02/01/2010 13:00 Test Status: APPROVED

HT Deadline: 03/13/2010 23:59 Date Analyzed: 02/15/2010 18:56

Lab Group: OYGCMS

\begin{tabular}{ll} 
Analyte Id & Analyte Name \\
\hline 120821 & 1,2,4-Trichlorobenzene \\
95501 & 1,2-Dichlorobenzene \\
122667 & 1,2-Diphenylhydrazine \\
541731 & 1,3-Dichlorobenzene \\
106467 & 1,4-Dichlorobenzene \\
95954 & 2,4,5-Trichloropheno \\
88062 & 2,4,6-Trichlorophenol \\
120832 & 2,4-Dichlorophenol \\
105679 & 2,4-Dimethylphenol \\
51285 & 2,4-Dinitrophenol \\
121142 & 2,4-Dinitrotoluene \\
606202 & 2,6-Dinitrotoluene \\
91587 & 2-Chloronaphthalene \\
95578 & 2-Chlorophenol \\
534521 & 2-Methyl-4,6-dinitrophenol \\
91576 & 2-Methylnaphthalene \\
95487 & 2-Methylphenol \\
88744 & 2-Nitrobenzenamine \\
88755 & 2-Nitrophenol \\
91941 & 3,3'-Dichlorobenzidine \\
N2799 & 3- and 4- Methylphenol \\
99092 & 3-Nitrobenzenamine \\
101553 & 4-Bromophenyl phenyl ether \\
59507 & 4-Chloro-3-methylphenol \\
106478 & 4-Chlorobenzenamine \\
7005723 & 4-Chlorophenylphenyl ether \\
100016 & 4-Nitrobenzenamine \\
100027 & 4-Nitrophenol \\
83329 & Acenaphthene \\
208968 & Acenaphthylene \\
62533 & Aniline \\
120127 & Anthracene \\
100516 & Benzenemethanol \\
92875 & Benzidine \\
56553 & Benzo(a)anthracene \\
50328 & Benzo(a)pyrene \\
205992 & Benzo(b)fluoranthene \\
191242 & Benzo(ghi)perylene \\
207089 & Benzo(k)fluoranthene \\
65850 & Benzoic acid \\
111911 & Bis(2-chloroethoxy)methane \\
111444 & Bis(2-chloroethyl) ether \\
108601 & Bis(2-chloroisopropy1) ether \\
117817 & Bis(2-ethylhexyl)phthalate \\
85687 & Butylbenzylphthalate \\
86748 & Carbazole \\
218019 & Chrysene \\
84742 & Di-n-butylphthalate \\
117840 & Di-n-octylphthalate \\
53703 & Dibenz(a,h)anthracene \\
&
\end{tabular}

\begin{tabular}{|c|c|c|c|c|c|c|c|c|}
\hline & stome & & Dilution & Lower & & EPA & & \\
\hline HT & Lim & Fn & Factor & Limit & Result & Qual & Confidence & Unit \\
\hline-- & $\cdots$ & -- & ----- & ---- & ---o- & $-\cdots$ & - & $-\cdot-$ \\
\hline & & & 3 & 300 & 1500 & u & & $\mathrm{ug} / \mathrm{L}$ \\
\hline & & & 3 & 340 & 1500 & u & & $\mathrm{ug} / \mathrm{L}$ \\
\hline & & & 3 & 180 & 1500 & $u$ & & $u g / L$ \\
\hline & & & 3 & 510 & 1500 & u & & $u g / L$ \\
\hline & & & 3 & 530 & 1500 & u & & ug/L \\
\hline & & & 3 & .580 & 1500 & u & & $\mathrm{ug} / \mathrm{L}$ \\
\hline & & & 3 & 310 & 1500 & u & & $\mathrm{ug} / \mathrm{L}$ \\
\hline & & & 3 & 290 & 1500 & U & & $u g / L$ \\
\hline & & & 3 & 300 & 1500 & u & & $u g / L$ \\
\hline & & & 3 & 430 & 3000 & u & & $\mathrm{ug} / \mathrm{L}$ \\
\hline & & & 3 & 390 & 1500 & u & & $\mathrm{ug} / \mathrm{L}$ \\
\hline & & & 3 & 290 & 1500 & $u$ & & ug $/ \mathrm{L}$ \\
\hline & & & 3 & 260 & 1500 & u & & $\mathrm{ug} / \mathrm{L}$ \\
\hline & & & 3 & 300 & 1500 & $u$ & & $\mathrm{ug} / \mathrm{L}$ \\
\hline & & & 3 & 320 & 3000 & u & & $\mathrm{ug} / \mathrm{L}$ \\
\hline & & & 3 & 200 & 1500 & U & & $\mathrm{ug} / \mathrm{L}$ \\
\hline & & & 3 & 290 & 1500 & $u$ & & $\mathrm{ug} / \mathrm{L}$ \\
\hline & & & 3 & 340 & 1500 & 0 & & $u g / L$ \\
\hline & & & 3 & 240 & 1500 & u & & ug/L \\
\hline & & & 3 & 150 & 3000 & u & & $\mathrm{ug} / \mathrm{L}$ \\
\hline & & & 3 & 350 & 3000 & $u$ & & ug/L \\
\hline & & & 3 & 650 & 1500 & u & & $\mathrm{ug} / \mathrm{L}$ \\
\hline & & & 3 & 200 & 1500 & $\mathrm{u}$ & & $\mathrm{ug} / \mathrm{L}$ \\
\hline & & & 3 & 160 & 1500 & $\mathrm{u}$ & & $\mathrm{ug} / \mathrm{L}$ \\
\hline & & & 3 & 300 & 1500 & 0 & & ug/L \\
\hline & & & 3 & 200 & 1500 & 0 & & $\mathrm{ug} / \mathrm{L}$ \\
\hline & & & 3 & 330 & 3000 & u & & $\mathrm{ug} / \mathrm{L}$ \\
\hline & & & 3 & 300 & 3000 & u & & $\mathrm{ug} / \mathrm{L}$ \\
\hline & & & 3 & 270 & 1500 & u & & ug $/ \mathrm{L}$ \\
\hline & & & 3 & 310 & 1500 & u & & $u g / L$ \\
\hline & & & 3 & 120 & 3000 & u & & $u g / L$ \\
\hline & & & 3 & 160 & 1500 & u & & $\mathrm{ug} / \mathrm{L}$ \\
\hline & & & 3 & 180 & 3000 & u & & $\mathrm{ug} / \mathrm{L}$ \\
\hline & & & 3 & 370 & 3000 & U & & $\mathrm{ug} / \mathrm{L}$ \\
\hline & & & 3 & 170 & 1500 & u & & $\mathrm{ug} / \mathrm{L}$ \\
\hline & & & 3 & 140 & 1500 & $\mathrm{u}$ & & $\mathrm{ug} / \mathrm{L}$ \\
\hline & & & 3 & 330 & 1500 & $\mathrm{u}$ & & ug/L \\
\hline & & & 3 & 450 & 1500 & $\mathrm{u}$ & & $\mathrm{ug} / \mathrm{L}$ \\
\hline & & & 3 & 440 & 1500 & u & & $\mathrm{ug} / \mathrm{L}$ \\
\hline & & & 3 & 570 & 3000 & u & & $\mathrm{ug} / \mathrm{L}$ \\
\hline & & & 3 & 160 & 1500 & $u$ & & $\mathrm{ug} / \mathrm{L}$ \\
\hline & & & 3 & 410 & 1500 & u & & $u g / L$ \\
\hline & & & 3 & 210 & 1500 & 0 & & $\mathrm{ug} / \mathrm{L}$ \\
\hline & & & 3 & 370 & 1500 & 0 & & $\mathrm{ug} / \mathrm{L}$ \\
\hline & & & 3 & 370 & 1500 & U & & $\mathrm{ug} / \mathrm{L}$ \\
\hline & & & 3 & 180 & 1500 & U & & $\mathrm{ug} / \mathrm{L}$ \\
\hline & & & 3 & 290 & 1500 & U & & $\mathrm{ug} / \mathrm{L}$ \\
\hline & & & 3 & 210 & 1500 & U & & $\mathrm{ug} / \mathrm{L}$ \\
\hline & & & 3 & 420 & 1500 & U & & $\mathrm{ug} / \mathrm{L}$ \\
\hline & & & 3 & 480 & 1500 & U & & $\mathrm{ug} / \mathrm{L}$ \\
\hline
\end{tabular}

UNCLASSIFIED 
UNCLASSIFIED

LIMS 03.08 .048

02/22/2010 07:03

132649 Dibenzofuran

84662 Diethylphthalate

131113 Dimethylphthalate

206440 Fluoranthene

86737 Fluorene

118741 Hexachlorobenzene

77474 Hexachlorocyclopentadiene

67721 Hexachloroethane

193395 Indeno(1,2,3-cd)pyrene

78591 Isophorone

$621647 \quad$ N-Nitroso-di-n-propylamine

$62759 \quad \mathrm{~N}-\mathrm{Nit}$ rosodimethylamine

$86306 \quad \mathrm{~N}$-Nitrosodiphenylamine

$91203 \quad$ Naphthalene

$98953 \quad$ Nitrobenzene

$87865 \quad$ Pentachlorophenol

$85018 \quad$ Phenanthrene

108952 Phenol

129000 Pyrene

\section{TIC Id TIC Name}

000000000

000403190

013402023 unknown acid hexadecanyl ester \$\$ 2-Prop

001794861 Phosgene oxime \$\$ phosgenox \$\$ CX \$\$ Hyd

074752946 Cyclopropane, 1-chloro-2-(1-propeny 1)-

002562370 cyclohexene, 1-nitro- \$\$ 1-Nitrocyclohex

007459714 3,5-Dimethylcyclopentene \$\$ 3,5-Dimethy?

000123422 2-Pentanone, 4-hydroxy-4-methyl- (CAS) \$

000000000 2-NITROPHENOL-D4

unknown

unknown

unknown

unknown

unknown

unknown

unknown

unknown

unknown

unknown
Analytical Chemistry organization (Quality Services)

P.O. Box 2009 oak Ridge, TN 378318244 (865) 574-2284

Page 2 of 2

Lab Smpl Id: A100250075

official Report

Cust Smpl Id: HFIR SRS $250 \mathrm{Mrad} 2 \mathrm{MNaOH}$

$1500 \quad u \quad u g / L$

$1500 \quad u \quad u g / L$

$1500 \quad U \quad u g / L$

$1500 \quad U \quad u g / L$

$\begin{array}{lll}1500 & U & \mathrm{ug} / \mathrm{L} \\ 1500 & U & \mathrm{ug} / \mathrm{L}\end{array}$

$1500 \quad U$ U ug/L

$1500 \quad u \quad u g / L$

$1500 \quad u \quad u g / L$

$1500 \quad u \quad u g / L$

$1500 \quad U \quad u g / L$

$1500 \quad u \quad$ ug/L

$1500 \quad u \quad u g / L$

$1500 \quad u \quad$ ug/L

$3000 \quad u \quad u g / L$

$1500-4$

$1500 \quad$ U

$\mathrm{ug} / \mathrm{L}$

Fn

Retention

Time unit

Estimated EPA

$\begin{array}{ll}\text { EStimated } & \text { EPA } \\ \text { Conc } & \text { Qua1 Unit }\end{array}$

$\begin{array}{lll}950 & ------\end{array}$

$1000-u g /$

$1000 \quad J N \quad u g / L$

$\begin{array}{lll}2200 & J & \mathrm{ug} / \mathrm{L} \\ 3400 & \mathrm{JN} & \mathrm{ug} / \mathrm{L}\end{array}$

$2700-3 N-200$

$1400 \quad J N \quad u g / L$

$6800 \quad$ JN $\quad$ ug/L

$3400 \quad J N \quad$ ug/L

$2400 \quad J N \quad$ ug/L

$2300-3 N \quad-\quad H g / L$

$1200 \quad J \quad$ ug/L

$2100 \quad \mathrm{ug} / \mathrm{L}$

$7200 \quad \mathrm{~J} \quad \mathrm{ug} / \mathrm{L}$

$920 \quad \mathrm{~J} \quad \mathrm{ug} / \mathrm{L}$

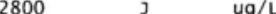

$5500 \quad J \quad \mathrm{ug} / \mathrm{L}$

$1900 \quad J \quad u g / L$

$720 \quad 3 \quad u g / L$

$1100 \quad \mathrm{~J} \quad \mathrm{ug} / \mathrm{L}$

1200

ug/L

Sample Test Comments: Target Compound(s) Initial Calibration exceeding 15\% RSD:

62-53-3 Aniline

207-08-9 Benzo [k]fluoranthene

The mean Initial calibration RSD $=8.7$

Comment objects:

EPA Qualifiers:

J - Estimated value.

$\mathrm{N}$ - Presumptive evidence of a compound. (GC/MS flag)

$U$ - Analyte analyzed for but undetected. Analyte result was below the contract required quantitation limit (CRQL).

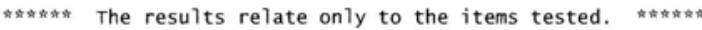

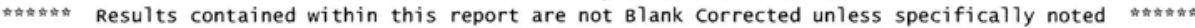

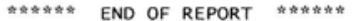

UNCLASSIFIED 
UNCLASSIFIED

LIMS 03.08 .048

$12 / 02 / 200913: 48$
Analytical Chemistry organization (Quality Services)

P.o. Box 2009 oak Ridge, TN 378318244 (865) 574-2284

official Report
Page 1 of 2

Lab Smp 1 Id: A093150153

Project: L MS MPO SCHUH SVOA

Proj Mgr: L P BURNETT (865) 576-9141

Customer: L P BURNETT Bldg 9995 MS-8189 (865) 576-9141

\begin{tabular}{|c|c|c|c|c|c|c|c|}
\hline Matrix & $\begin{array}{l}\text { Chain of } \\
\text { Custody }\end{array}$ & $\begin{array}{l}\text { Work Agreement } \\
\text { Number }\end{array}$ & $\begin{array}{l}\text { Charge } \\
\text { Number }\end{array}$ & $\begin{array}{l}\text { Date } \\
\text { Sampled }\end{array}$ & $\begin{array}{l}\text { Date } \\
\text { Received }\end{array}$ & $\begin{array}{l}\text { Date } \\
\text { Needed }\end{array}$ & $\begin{array}{l}\text { Date } \\
\text { Approved }\end{array}$ \\
\hline - - & -...-. & $-\ldots$ & $\ldots$ & - & - n-n-- & - & - \\
\hline LIQUID & 117883 & 81473 & $745 A 0221$ & $11 / 10 / 200900: 00$ & $11 / 10 / 2009 \quad 15: 30$ & $12 / 09 / 2009 \quad 23: 59$ & $12 / 02 / 200913: 48$ \\
\hline
\end{tabular}

Sample Description:

Location: ORNL

Sample Status: APPROVED

Sampler(s):

Customer Comments:

Lab Comments:

Comment objects :

$\ll<<$ Organic $\gg \gg \gg$ Test: SVOA8270 (Semi-volatile organics by GC/MS)

Rpt Basis: AR Test Req Cnt: 01 Analysis Method: SW846 8270C (Y50-AC-65-7316)

Approver: I T HOFFARTH/Data Approver QC Batch/File: QC09336003/5206/53706
Prep Method/Date: SW846 3580A 11/18/2009 14:00 Test Status: APPROVED

HT Deadline: $12 / 28 / 2009$ 23:59 Date Analyzed: 11/30/2009 18:23

Lab Group: OYGCMS

\begin{tabular}{|c|c|}
\hline Ana7yte Id & Analyte Name \\
\hline 120821 & 1,2,4-Trich1orobenzene \\
\hline 95501 & 1,2-Dichlorobenzene \\
\hline 122667 & 1,2-Diphenylhydrazine \\
\hline 541731 & 1,3-Dichlorobenzene \\
\hline 106467 & 1,4-Dichlorobenzene \\
\hline 95954 & 2,4,5-Trichlorophenol \\
\hline 88062 & 2,4,6-Trichlorophenol \\
\hline 120832 & 2,4-Dichlorophenol \\
\hline 105679 & 2,4-Dimethylphenol \\
\hline 51285 & 2,4-Dinitrophenol \\
\hline 121142 & 2,4-Dinitrotoluene \\
\hline 606202 & 2,6-Dinitrotoluene \\
\hline 91587 & 2-Chloronaphthalene \\
\hline 95578 & 2-Ch1orophenol \\
\hline 534521 & 2-Methy $1-4,6$-dinitropheno 1 \\
\hline 91576 & 2-Methylnaphthalene \\
\hline 95487 & 2-Methylphenol \\
\hline 88744 & 2-Nitrobenzenamine \\
\hline 88755 & 2-Nitrophenol \\
\hline 91941 & $3,3^{\prime}$-Dichlorobenzidine \\
\hline N2799 & $3-$ and 4- Methylphenol \\
\hline 99092 & 3-Nitrobenzenamine \\
\hline 101553 & 4-Bromopheny 1 pheny 1 ether \\
\hline 59507 & 4-chloro-3-methylphenol \\
\hline 106478 & 4-Chlorobenzenamine \\
\hline 7005723 & 4-Chloropheny 1 pheny 1 ether \\
\hline 100016 & 4-Nitrobenzenamine \\
\hline 100027 & 4-Nitrophenol \\
\hline 83329 & Acenaphthene \\
\hline 208968 & Acenaphthylene \\
\hline 62533 & Aniline \\
\hline 120127 & Anthracene \\
\hline 100516 & Benzenemethanol \\
\hline 92875 & Benzidine \\
\hline 56553 & Benzo(a)anthracene \\
\hline 50328 & Benzo (a)pyrene \\
\hline 205992 & Benzo (b) fluoranthene \\
\hline 191242 & Benzo(ghi)perylene \\
\hline 207089 & Benzo $(k)$ fluoranthene \\
\hline 65850 & Benzoic acid \\
\hline 111911 & Bis (2-chloroethoxy)methane \\
\hline 111444 & Bis (2-chloroethyl) ether \\
\hline 108601 & Bis (2-chloroisopropy 1 ) ether \\
\hline 117817 & Bis (2-ethylhexy1)phthalate \\
\hline 85687 & Butylbenzylphthalate \\
\hline 86748 & Carbazole \\
\hline 218019 & Chrysene \\
\hline 84742 & Di-n-butylphthalate \\
\hline 117840 & Di-n-octylphthalate \\
\hline 53703 & Dibenz $(a, h)$ anthracene \\
\hline
\end{tabular}

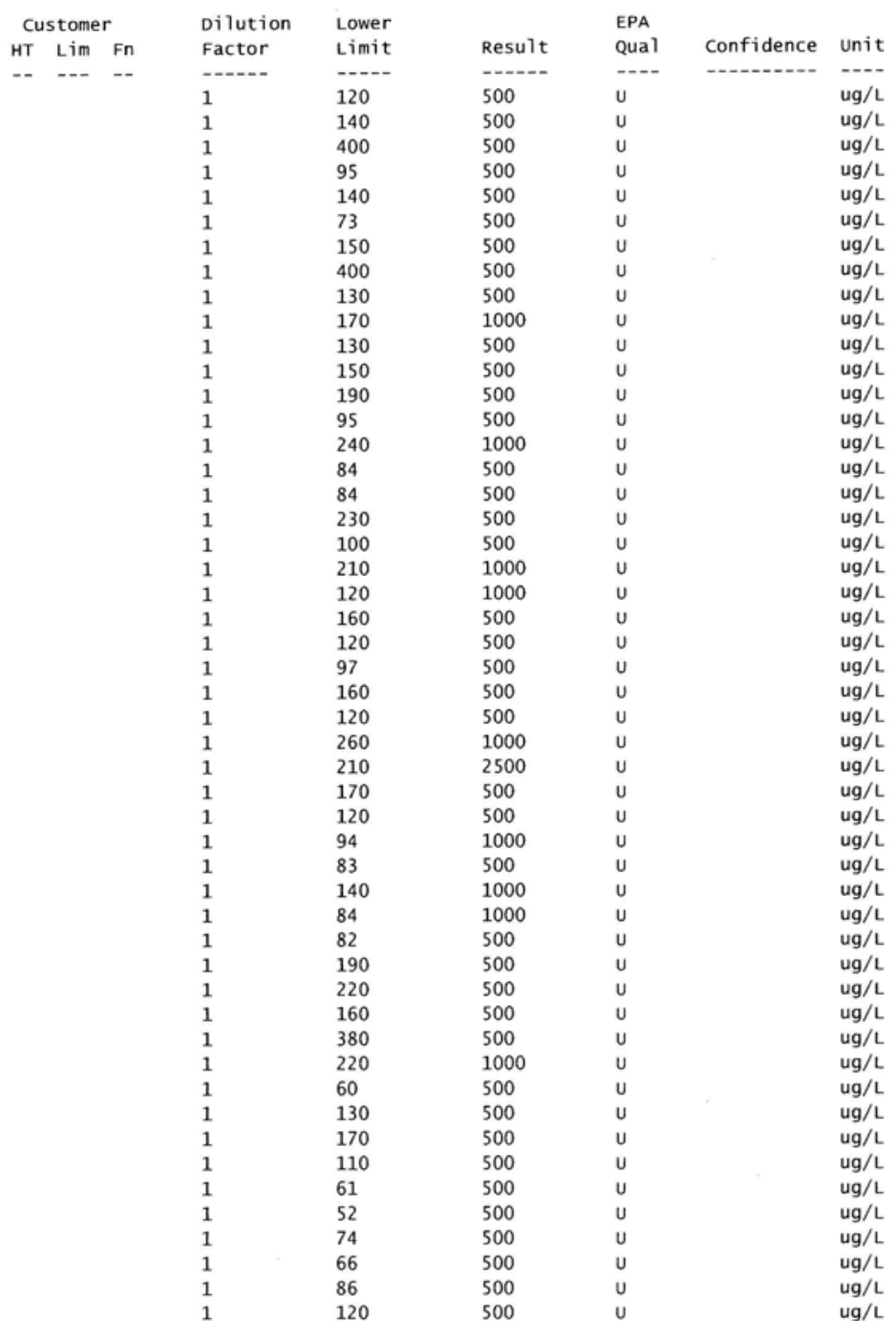

UNCLASSIFIED 
UNCLASSIFIED

LIMS 03.08.048

12/02/2009 13:48

132649 Dibenzofuran

84662 Diethylphthalate

131113 Dimethylphthalate

$206440 \quad$ Fluoranthene

86737 Fluorene

118741 Hexachlorobenzene

77474 Hexachlorocyclopentadiene

67721 Hexachloroethane

193395 Indeno $(1,2,3-c d)$ pyrene

78591 Isophorone

$621647 \quad$ N-Nitroso-di-n-propylamine

$62759 \quad \mathrm{~N}$-Nitrosodimethylamine

$86306 \quad \mathrm{~N}$-Nitrosodiphenylamine

91203 Naphthalene

$98953 \quad$ Nitrobenzene

87865 Pentachlorophenol

$85018 \quad$ Phenanthrene

108952 Phenol

129000 Pyrene

TIC Id TIC Name

- 000870633 - 2 - Butene, $1-$ bromo-3-methy

000870633 2-Butene, 1-bromo-3-methy1-\$\$ .gamma.,. unknown alcohol

unknown

000870633 2-Butene, 1-bromo-3-methy1-(CAS) \$\$ .ga unknown

unknown alcohol

000870633 2-Butene, 1-bromo-3-methyl- (CAS) \$\$ .ga

unknown

unknown

unknown

unknown

unknown

unknown

unknown

unknown

unknown

unknown

unknown
Analytical Chemistry organization (Quality Services)

Page 2 of 2

P.O. Box 2009 oak Ridge, TN 378318244 (865) 574-2284

Lab Smp1 Id: A093150153

official Report

Cust Smpl Id: SRS $200 \mathrm{Mrad}$

$\begin{array}{ll}1 & 7 \\ 1 & 1 \\ 1 & 1 \\ 1 & 1 \\ 1 & 1 \\ 1 & 1 \\ 1 & 1 \\ 1 & 60 \\ 1 & 1 \\ 1 & 7 \\ 1 & 1 \\ 1 & 290 \\ 1 & 130 \\ 1 & 77 \\ 1 & 1 \\ 1 & 180 \\ 1 & 51 \\ 1 & 65 \\ 1 & 130\end{array}$

\begin{tabular}{|c|c|}
\hline 78 & 500 \\
\hline 120 & 500 \\
\hline 100 & 500 \\
\hline 120 & 500 \\
\hline 100 & 500 \\
\hline 140 & 500 \\
\hline 180 & 500 \\
\hline 60 & 500 \\
\hline 110 & 500 \\
\hline 76 & 500 \\
\hline 160 & 500 \\
\hline 290 & 500 \\
\hline 130 & 500 \\
\hline 77 & 500 \\
\hline 110 & 500 \\
\hline 180 & 1000 \\
\hline 1 & 500 \\
\hline & 500 \\
\hline 130 & 500 \\
\hline
\end{tabular}

Fn

Retention

Time

6.71

6.71
7.17

9.36

10.36

6.02

11.23

9.49

7

11.59

12.23

12.35

12.75

13.09

6.31

7.7

8.03

8.13

8.25
8.48

Unit
min
min
min
min
min
min
min
min
min
min
min
$\min$
$\min$
$\min$
$\min$
min
$\min$
$\min$
$\min$

\begin{tabular}{|c|c|c|}
\hline$u$ & & $u g / L$ \\
\hline u & & $u g / L$ \\
\hline U & & $u g / L$ \\
\hline$u$ & & $u g / L$ \\
\hline u & & $u g / L$ \\
\hline U & & ug/L \\
\hline u & & $\mathrm{ug} / \mathrm{L}$ \\
\hline u & & $\mathrm{ug} / \mathrm{L}$ \\
\hline u & & $\mathrm{ug} / \mathrm{L}$ \\
\hline u & & $u g / L$ \\
\hline u & & $u g / L$ \\
\hline u & & $u g / L$ \\
\hline u & & $u g / L$ \\
\hline u & & $u g / L$ \\
\hline u & & $u g / L$ \\
\hline u & & $u g / L$ \\
\hline u & & $\mathrm{ug} / \mathrm{L}$ \\
\hline U & & $u g / L$ \\
\hline u & & $u g / L$ \\
\hline Estimated & EPA & \\
\hline Conc & Qual & Unit \\
\hline -....- & $--\cdot$ & $\cdots$ \\
\hline 1700 & JN & $\mathrm{ug} / \mathrm{L}$ \\
\hline 2300 & JN & $\mathrm{ug} / \mathrm{L}$ \\
\hline 1600 & 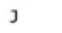 & $\mathrm{ug} / \mathrm{L}$ \\
\hline 4600 & $J$ & ug $/ \mathrm{t}$ \\
\hline 16000 & JN & $\mathrm{ug} / \mathrm{L}$ \\
\hline 7200 & J & $u g / L$ \\
\hline 9500 & J & $u g / L$ \\
\hline 16000 & JN & $u g / L$ \\
\hline 2700 & $\mathrm{~J}$ & $u g / L$ \\
\hline 6800 & J & $u g / L$ \\
\hline 1700 & J & $u g / L$ \\
\hline 3400 & J & $u g / L$ \\
\hline 3800 & J & $u g / L$ \\
\hline 2300 & 了 & $u g / L$ \\
\hline 2300 & J & $u g / L$ \\
\hline 7900 & J & $u g / L$ \\
\hline 2000 & J & $\mathrm{ug} / \mathrm{L}$ \\
\hline 1300 & J & $\mathrm{ug} / \mathrm{L}$ \\
\hline 8800 & J & $u g / L$ \\
\hline
\end{tabular}

Sample Test Comments: Target Compound(s) Initial Calibration exceeding 15\% RSD: $111-44-4$ The mean Initial Calibration RSD $=9.3$

Comment objects:

EPA Qualifiers:

J - Estimated value.

$\mathrm{N}$ - Presumptive evidence of a compound. (GC/MS flag)

U - Analyte analyzed for but undetected. Analyte result was below the contract required quantitation limit (CRQL).

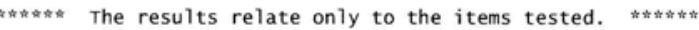

해

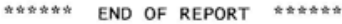

UNCLASSIFIED 
UNCLASSIFIED

LIMS 03.08.048

12/02/2009 13:46

Analytical Chemistry organization (Quality Services)

Page 1 of 2

P.o. Box 2009 oak Ridge, TN 378318244 (865) 574-2284

Lab Smp1 Id: A093150152

Project: L MS MPO SCHUH SVOA

official Report

Cust Smpl Id: SRS 150 Mrad

Proj Mgr: L P BURNETT (865) 576-9141

Customer: L P BURNETT Bldg 9995 MS-8189 (865) 576-9141

\begin{tabular}{|c|c|c|c|c|c|c|c|}
\hline Matrix & $\begin{array}{l}\text { Chain of } \\
\text { Custody }\end{array}$ & $\begin{array}{l}\text { Work Agreement } \\
\text { Number }\end{array}$ & $\begin{array}{l}\text { Charge } \\
\text { Number }\end{array}$ & $\begin{array}{l}\text { Date } \\
\text { Sampled }\end{array}$ & $\begin{array}{l}\text { Date } \\
\text { Received }\end{array}$ & $\begin{array}{l}\text { Date } \\
\text { Needed }\end{array}$ & $\begin{array}{l}\text { Date } \\
\text { Approved }\end{array}$ \\
\hline -...-- & -...- & -...-- & -.--- & - & $-\cdots$ & - & - \\
\hline LIQUID & 117883 & 81473 & $745 A 0221$ & $11 / 10 / 200900: 00$ & $11 / 10 / 200915: 30$ & $12 / 09 / 2009 \quad 23: 59$ & $12 / 02 / 2009 \quad 13: 46$ \\
\hline
\end{tabular}

Sample Description:

Location: ORNL Sample Approver: J T HOFFARTH/Data APprover

Customer comments:

Lab Comments:

Comment objects:

Sampler(s):

$\ll<<$ Organic $\gg \gg>$

Test: SVOA8270 (Semi-Volatile Organics by GC/MS) Test

Analysis Method: SW846 8270C (Y50-AC-65-7316)

Approver: J T HOFFARTH/Data Approver

QC Batch/File: QC09336003/5206/53706

Prep Method/Date: SW846 3580A 11/18/2009 14:00 Test Status: APPROVED

HT Deadline: 12/28/2009 23:59

Date Analyzed: 11/30/2009 17:39

Lab Group: OYGCMS

\begin{tabular}{|c|c|}
\hline Analyte Id & Analyte Name \\
\hline 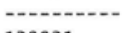 & - \\
\hline 120821 & 1,2,4-Trichlorobenzene \\
\hline 95501 & 1,2-Dich7orobenzene \\
\hline 122667 & 1,2-Diphenylhydrazine \\
\hline 541731 & 1,3-Dichlorobenzene \\
\hline 106467 & 1,4-Dichlorobenzene \\
\hline 95954 & 2,4,5-Trichlorophenol \\
\hline 88062 & $2,4,6$-Trichlorophenol \\
\hline 120832 & 2,4-Dich1orophenol \\
\hline 105679 & 2,4-Dimethylphenol \\
\hline 51285 & 2,4-Dinitrophenol \\
\hline 121142 & 2,4-Dinitrotoluene \\
\hline 606202 & 2,6 -Dinit rotoluene \\
\hline 91587 & 2-chloronaphthalene \\
\hline 95578 & 2-Ch1orophenol \\
\hline 534521 & 2-Methy1-4,6-dinitrophenol \\
\hline 91576 & 2-Methylnaphthalene \\
\hline 95487 & 2-Methylphenol \\
\hline 88744 & 2-Nitrobenzenamine \\
\hline 88755 & 2-Nitrophenol \\
\hline 91941 & 3, $3^{\prime}$-Dichlorobenzidine \\
\hline N2799 & 3- and 4- Methylphenol \\
\hline 99092 & 3-Nitrobenzenamine \\
\hline 101553 & 4-Bromophenyl phenyl ether \\
\hline 59507 & 4-Chloro-3-methylphenol \\
\hline 106478 & 4-Chlorobenzenamine \\
\hline 7005723 & 4-Ch1oropheny 1 pheny1 ether \\
\hline 100016 & 4-Nitrobenzenamine \\
\hline 100027 & 4-Nitrophenol \\
\hline 83329 & Acenaphthene \\
\hline 208968 & Acenaphthylene \\
\hline 62533 & Aniline \\
\hline 120127 & Anthracene \\
\hline 100516 & Benzenemethanol \\
\hline 92875 & Benzidine \\
\hline 56553 & Benzo (a)anthracene \\
\hline 50328 & Benzo (a)pyrene \\
\hline 205992 & Benzo(b)fluoranthene \\
\hline 191242 & Benzo(ghi)perylene \\
\hline 207089 & Benzo $(k)$ fluoranthene \\
\hline 65850 & Benzoic acid \\
\hline 111911 & Bis (2-chloroethoxy)methane \\
\hline 111444 & Bis (2-chloroethyl) ether \\
\hline 108601 & Bis (2-chloroisopropyl) ether \\
\hline 117817 & Bis(2-ethylhexy1)phthalate \\
\hline 85687 & Butylbenzylphthalate \\
\hline 86748 & Carbazole \\
\hline 218019 & Chrysene \\
\hline 84742 & Di-n-butylphthalate \\
\hline 117840 & Di-n-octylphthalate \\
\hline 53703 & Dibenz $(a, h)$ anthracene \\
\hline
\end{tabular}

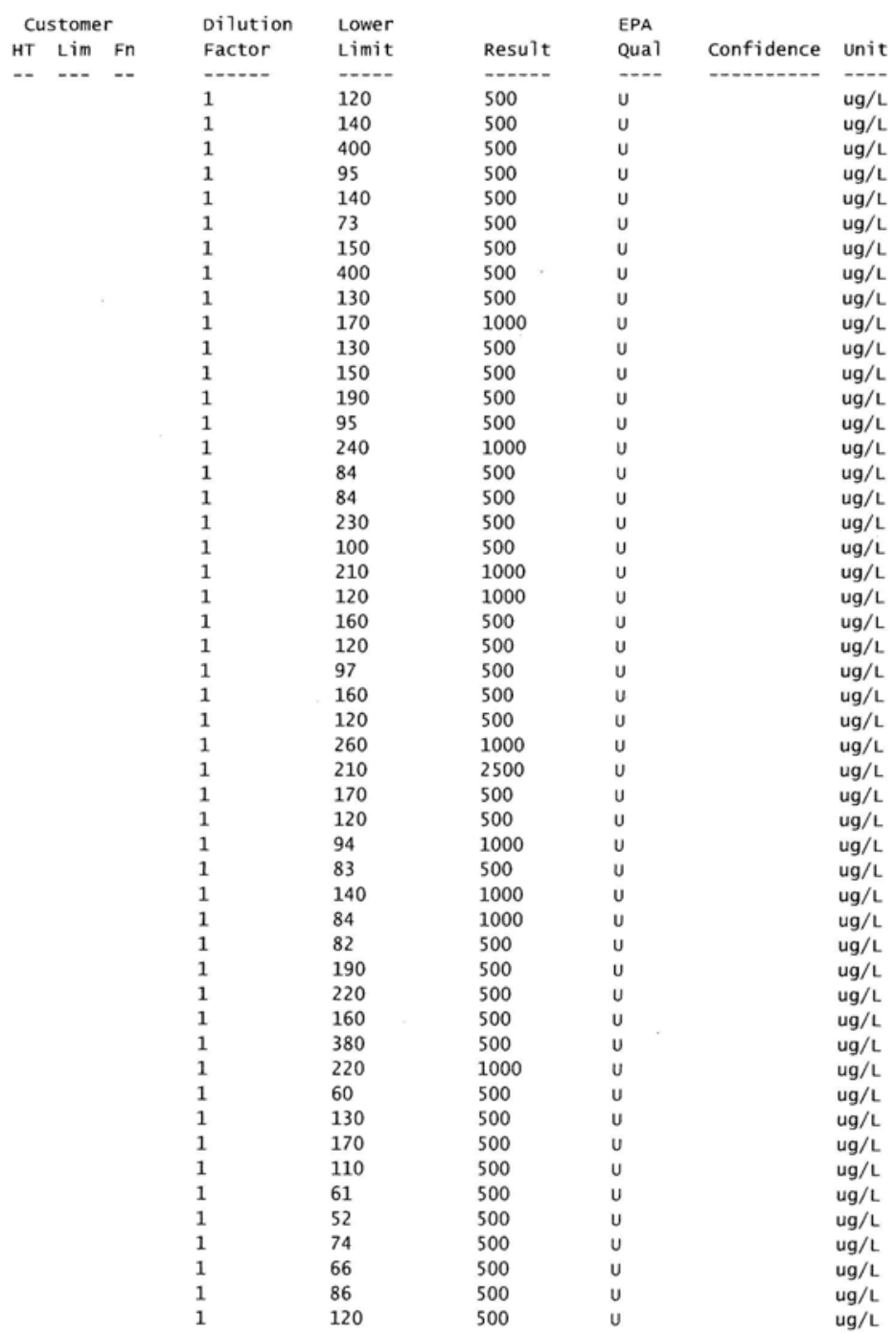

UNCLASSIFIED 


\section{UNCLASSIFIED}

LIMS 03.08 .048

12/02/2009 13:46

\begin{tabular}{|c|c|}
\hline 132649 & Dibenzofuran \\
\hline 84662 & Diethylphthalate \\
\hline 131113 & Dimethylphthalate \\
\hline 206440 & Fluoranthene \\
\hline 86737 & Fluorene \\
\hline 118741 & Hexachlorobenzene \\
\hline 77474 & Hexachlorocyclopentadiene \\
\hline 67721 & Hexachloroethane \\
\hline 193395 & Indeno $(1,2,3-\mathrm{cd})$ pyrene \\
\hline 78591 & Isophorone \\
\hline 621647 & $\mathrm{~N}$-Nitroso-di-n-propylamine \\
\hline 62759 & $\mathrm{~N}$-Nitrosodimethylamine \\
\hline 86306 & $\mathrm{~N}$-Nit rosodiphenylamine \\
\hline 91203 & Naphthalene \\
\hline 98953 & Nitrobenzene \\
\hline 87865 & Pentachlorophenol \\
\hline 85018 & Phenanthrene \\
\hline 108952 & Phenol \\
\hline 129000 & Pyrene \\
\hline TIC Id & TIC Name \\
\hline - n- & 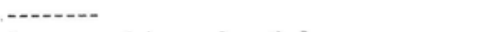 \\
\hline 000870633 & 2-Butene, 1-bromo-3-methy1- \\
\hline 000870633 & $\begin{array}{l}\text { 2-Butene, 1-bromo-3-methyl- } \$ \$ \text {.gamma., } \\
\text { unknown alcohol/alkoxy cpd } \\
\text { unknown alcohol } \\
\text { unknown }\end{array}$ \\
\hline 000123422 & $\begin{array}{l}\text { 2-Pentanone, 4-hydroxy-4-methy1- (CAS) \$ } \\
\text { unknown }\end{array}$ \\
\hline 000870633 & $\begin{array}{l}\text { 2-Butene, 1-bromo-3-methy1- \$\$ .gamma.,. } \\
\text { unknown alcohol/alkoxy cpd } \\
\text { unknown }\end{array}$ \\
\hline 000870633 & $\begin{array}{l}\text { 2-Butene, 1-bromo-3-methy1- } \$ \$ . \text { gamma.,. } \\
\text { unknown } \\
\text { unknown } \\
\text { unknown } \\
\text { unknown } \\
\text { unknown } \\
\text { unknown } \\
\text { unknown } \\
\text { unknown } \\
\text { unknown }\end{array}$ \\
\hline
\end{tabular}

Analytical Chemistry organization (Quality Services) P.o. Box 2009 oak Ridge, TN 378318244 (865) 574-2284

Page 2 of 2

official Report Cust Smpl Id: SRS 150 Mrad

official Report Cust Smpl Id: SRS 150 Mrad

Lab Smp1 Id: A093150152

\begin{tabular}{|c|c|c|c|c|c|c|}
\hline & 1 & 78 & 500 & \multicolumn{2}{|l|}{ u } & $\mathrm{ug} / \mathrm{L}$ \\
\hline & 1 & 120 & 500 & \multicolumn{2}{|l|}{ u } & $u g / L$ \\
\hline & 1 & 100 & 500 & \multicolumn{2}{|l|}{ u } & $u g / L$ \\
\hline & 1 & 120 & 500 & \multicolumn{2}{|l|}{ u } & $\mathrm{ug} / \mathrm{L}$ \\
\hline & 1 & 100 & 500 & \multicolumn{2}{|l|}{ u } & $\mathrm{ug} / \mathrm{L}$ \\
\hline & 1 & 140 & 500 & \multicolumn{2}{|l|}{ u } & $u g / L$ \\
\hline & 1 & 180 & 500 & \multicolumn{2}{|l|}{$u$} & $\mathrm{ug} / \mathrm{L}$ \\
\hline & 1 & 60 & 500 & \multicolumn{2}{|l|}{$u$} & $\mathrm{ug} / \mathrm{L}$ \\
\hline & 1 & 110 & 500 & \multicolumn{2}{|l|}{$u$} & $\mathrm{ug} / \mathrm{L}$ \\
\hline & 1 & 76 & 500 & \multicolumn{2}{|l|}{$u$} & $u g / L$ \\
\hline & 1 & 160 & 500 & \multicolumn{2}{|l|}{ u } & $\mathrm{ug} / \mathrm{L}$ \\
\hline & 1 & 290 & 500 & \multicolumn{2}{|l|}{ u } & $\mathrm{ug} / \mathrm{L}$ \\
\hline & 1 & 130 & 500 & \multicolumn{2}{|l|}{$u$} & $\mathrm{ug} / \mathrm{L}$ \\
\hline & 1 & 77 & 500 & \multicolumn{2}{|l|}{ u } & $u g / L$ \\
\hline & 1 & 110 & 500 & \multicolumn{2}{|l|}{ u } & $u g / L$ \\
\hline & 1 & 180 & 1000 & \multicolumn{2}{|l|}{ u } & $\mathrm{ug} / \mathrm{L}$ \\
\hline & 1 & 51 & 500 & \multicolumn{2}{|l|}{$u$} & $\mathrm{ug} / \mathrm{L}$ \\
\hline & 1 & 65 & 500 & \multicolumn{2}{|l|}{ u } & $\mathrm{ug} / \mathrm{L}$ \\
\hline & 1 & 130 & 500 & \multicolumn{3}{|r|}{$\mathrm{ug} / \mathrm{L}$} \\
\hline & & Reten & & \multirow{2}{*}{$\begin{array}{l}\text { Estimated } \\
\text { Conc }\end{array}$} & \multicolumn{2}{|l|}{ EPA } \\
\hline \multicolumn{2}{|l|}{$\mathrm{Fn}$} & Time & Unit & & Qual & Unit \\
\hline \multirow[t]{15}{*}{--} & & --- & -... & ----- & --- & -- \\
\hline & & 6.69 & $\min$ & 1200 & JN & ug/L \\
\hline & & 5.95 & $\min$ & 10000 & JN & $\mathrm{ug} / \mathrm{L}$ \\
\hline & & 9.55 & $\min$ & 3200 & $J$ & $\mathrm{ug} / \mathrm{L}$ \\
\hline & & 9.38 & $\min$ & 8100 & J & $\mathrm{ug} / \mathrm{L}$ \\
\hline & & 10.31 & $\min$ & 2600 & $J$ & $\mathrm{ug} / \mathrm{L}$ \\
\hline & & 5.82 & $\min$ & 1900 & JN & $\mathrm{ug} / \mathrm{L}$ \\
\hline & & 11.17 & $\min$ & 2200 & $J$ & $\mathrm{ug} / \mathrm{L}$ \\
\hline & & 6.95 & $\min$ & 12000 & JN & $\mathrm{ug} / \mathrm{L}$ \\
\hline & & 9.64 & $\min$ & 1100 & J & $\mathrm{ug} / \mathrm{L}$ \\
\hline & & 11.56 & $\min$ & 790 & J & $u g / L$ \\
\hline & & 7.13 & $\min$ & 1700 & JN & $\mathrm{ug} / \mathrm{L}$ \\
\hline & & 12.17 & $\min$ & 2000 & $J$ & $u g / L$ \\
\hline & & 13.06 & $\min$ & 1600 & J & $\mathrm{ug} / \mathrm{L}$ \\
\hline & & 13.18 & $\min$ & 1000 & J & $u g / L$ \\
\hline \multirow[t]{6}{*}{, } & & 5.01 & $\min$ & 2400 & J & $\mathrm{ug} / \mathrm{L}$ \\
\hline & & 6.29 & $\min$ & 1500 & J & $u g / L$ \\
\hline & & 7.99 & $\min$ & 5500 & J & $\mathrm{ug} / \mathrm{L}$ \\
\hline & & 8.12 & $\min$ & 1100 & J & $u g / L$ \\
\hline & & 8.2 & $\min$ & 890 & J & $u g / L$ \\
\hline & & 8.42 & $\min$ & 6200 & J & $u g / L$ \\
\hline
\end{tabular}

Sample Test comments: Target Compound(s) Initial Calibration exceeding 15\% RSD: bis $(2-$ Chloroethy 1 ) ether
The mean Initial Calibration RSD $=9.3$

Comment objects:

EPA Qualifiers:

J - Estimated value.

N - Presumptive evidence of a compound. (GC/MS flag)

U - Analyte analyzed for but undetected. Analyte result was below the contract required quantitation limit (CRQL).

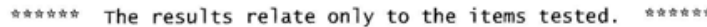

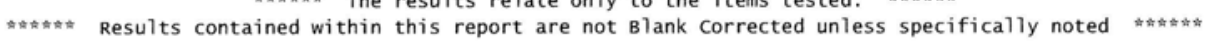

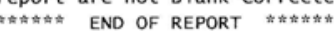

\section{UNCLASSIFIED}




\section{UNCLASSIFIED}

LIMS 03.08 .048

03/12/2010 16:03

Project: L MS MPO SCHUH SVOA
Analytical Chemistry organization (Quality Services)

P.o. Box 2009 oak Ridge, TN 378318244 (865) 574-2284

official Report
Page 1 of 2

Lab Smp1 Id: A100550084

Customer: L P BURNETT Bldg 9995 MS-8189 (865) 576-9141

\begin{tabular}{|c|c|c|c|c|c|c|c|}
\hline Matrix & $\begin{array}{l}\text { Chain of } \\
\text { Custody }\end{array}$ & $\begin{array}{l}\text { Work Agreement } \\
\text { Number }\end{array}$ & $\begin{array}{l}\text { Charge } \\
\text { Number }\end{array}$ & $\begin{array}{l}\text { Date } \\
\text { Sampled }\end{array}$ & $\begin{array}{l}\text { Date } \\
\text { Received }\end{array}$ & $\begin{array}{l}\text { Date } \\
\text { Needed }\end{array}$ & $\begin{array}{l}\text { Date } \\
\text { Approved }\end{array}$ \\
\hline --n-- & ----- & ----- & $\cdots-$ & $-\cdots$ & ----- & ----- & - - n-- \\
\hline LIQUID & 117936 & 81473 & $745 A 0221$ & $02 / 23 / 2010 \quad 00: 00$ & $02 / 23 / 2010 \quad 14: 30$ & 03/09/2010 23:59 & $03 / 12 / 2010$ \\
\hline
\end{tabular}

Customer Comments:

Lab Comments:

Comment objects:

Cust Smpl Id: 300 Mrad Hanford Sim

Proj Mgr: L P BURNETT (865) 576-9141

Sample Status: APPROVED
Sample Approver: J T HOFFARTH/Data Approver

$\ll<<$ organic $\gg \gg>$

Rpt Basis: AR Test Req Cnt: 01

Analysis Method: SW 8468270 C (Y50-AC-65-7316)
Approver: J T HOFFARTH/Data Approver

QC Batch/File: QC10070010/5314/53787
Prep Method/Date: SW846 3580A 02/24/2010 13:00 Test Status: APPROVED

HT Deadline: 04/05/2010 23:59

Date Analyzed: 03/10/2010 19:22 Lab Group: OYGCMS

\begin{tabular}{|c|c|}
\hline Analyte Id & Analyte Name \\
\hline -120821 & - \\
\hline $\begin{array}{l}120821 \\
95501\end{array}$ & 1,2,4-Trichlorobenzene \\
\hline 95501 & 1,2-Dichlorobenzene \\
\hline 122667 & 1,2-Diphenylhydrazine \\
\hline 541731 & 1,3-Dichlorobenzene \\
\hline 106467 & 1,4-Dichlorobenzene \\
\hline 95954 & 2,4,5-Trichlorophenol \\
\hline 88062 & $2,4,6$-Trichlorophenol \\
\hline 120832 & 2,4-Dichlorophenol \\
\hline 105679 & 2,4-Dimethylphenol \\
\hline 51285 & 2,4-Dinitrophenol \\
\hline 121142 & 2,4-Dinitrotoluene \\
\hline 606202 & 2,6-Dinitrotoluene \\
\hline 91587 & 2-Chloronaphthalene \\
\hline 95578 & 2-Ch1oropheno 1 \\
\hline 534521 & 2-Methy $7-4,6$-dinitrophenol \\
\hline 91576 & 2-Methylnaphthalene \\
\hline 95487 & 2-Methylphenol \\
\hline 88744 & 2-Nitrobenzenamine \\
\hline 88755 & 2-Nitrophenol \\
\hline 91941 & $3,3^{\prime}$-Dich 7 orobenzidine \\
\hline N2799 & 3- and 4- Methylphenol \\
\hline 99092 & 3-Nitrobenzenamine \\
\hline 101553 & 4-Bromopheny 1 pheny 1 ether \\
\hline 59507 & 4-chloro-3-methylphenol \\
\hline 106478 & 4-Chlorobenzenamine \\
\hline 7005723 & 4-Ch 1 oropheny 1 pheny 1 ether \\
\hline 100016 & 4-Nitrobenzenamine \\
\hline 100027 & 4-Nitrophenol \\
\hline 83329 & Acenaphthene \\
\hline 208968 & Acenaphthylene \\
\hline 62533 & Aniline \\
\hline 120127 & Anthracene \\
\hline 100516 & Benzeneme thanol \\
\hline 92875 & Benzidine \\
\hline 56553 & Benzo (a) anthracene \\
\hline 50328 & Benzo(a)pyrene \\
\hline 205992 & Benzo (b) fluoranthene \\
\hline 191242 & Benzo(ghi)perylene \\
\hline 207089 & Benzo $(k)$ fluoranthene \\
\hline 65850 & Benzoic acid \\
\hline 111911 & Bis (2-chloroethoxy)methane \\
\hline 111444 & Bis (2-chloroethy 1 ) ether \\
\hline 108601 & Bis (2-chloroisopropyl) ether \\
\hline 117817 & Bis(2-ethylhexy1)phthalate \\
\hline 85687 & Butylbenzylphthalate \\
\hline 86748 & Carbazole \\
\hline 218019 & Chrysene \\
\hline 84742 & Di-n-butylphthalate \\
\hline 117840 & Di-n-octylphthalate \\
\hline 53703 & Dibenz $(\mathrm{a}, \mathrm{h})$ anthracene \\
\hline
\end{tabular}

\begin{tabular}{|c|c|c|c|c|c|c|c|c|}
\hline \multicolumn{3}{|c|}{ Customer } & \multirow{2}{*}{$\begin{array}{l}\text { Dilution } \\
\text { Factor }\end{array}$} & \multirow{2}{*}{$\begin{array}{l}\text { Lower } \\
\text { Limit }\end{array}$} & \multirow[b]{2}{*}{ Result } & \multirow{2}{*}{$\begin{array}{l}\text { EPA } \\
\text { Qual }\end{array}$} & \multirow[b]{2}{*}{ Confidence } & \multirow[b]{2}{*}{ Unit } \\
\hline HT & Lim & $\mathrm{Fn}$ & & & & & & \\
\hline-- & $\cdots$ & -- & ---o- & $-\cdots$ & -and- & --- & 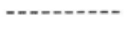 & --- \\
\hline & & & 1 & 99 & 500 & u & & $\mathrm{ug} / \mathrm{L}$ \\
\hline & & & 1 & 110 & 500 & $u$ & & $u g / L$ \\
\hline & & & 1 & 59 & 500 & $u$ & & $u g / L$ \\
\hline & & & 1 & 170 & 500 & $u$ & & $\mathrm{ug} / \mathrm{L}$ \\
\hline & & & 1 & 180 & 500 & $u$ & & $u g / L$ \\
\hline & & & 1 & 190 & 500 & $u$ & & $\mathrm{ug} / \mathrm{L}$ \\
\hline & & & 1 & 100 & 500 & $u$ & & $\mathrm{ug} / \mathrm{L}$ \\
\hline & & & 1 & 98 & 500 & $u$ & & $\mathrm{ug} / \mathrm{L}$ \\
\hline & & & 1 & 99 & 500 & u & & $\mathrm{ug} / \mathrm{L}$ \\
\hline & & & 1 & 140 & 1000 & u & & $\mathrm{ug} / \mathrm{L}$ \\
\hline & & & 1 & 130 & 500 & u & & $\mathrm{ug} / \mathrm{L}$ \\
\hline & & & 1 & 97 & 500 & $u$ & & $\mathrm{ug} / \mathrm{L}$ \\
\hline & & & 1 & 87 & 500 & u & & $\mathrm{ug} / \mathrm{L}$ \\
\hline & & & 1 & 100 & 500 & u & & $\mathrm{ug} / \mathrm{L}$ \\
\hline & & & 1 & 110 & 1000 & $u$ & & $u g / L$ \\
\hline & & & 1 & 66 & 500 & U & & $\mathrm{ug} / \mathrm{L}$ \\
\hline & & & 1 & 98 & 500 & u & & $u g / L$ \\
\hline & & & 1 & 110 & 500 & 0 & & $\mathrm{ug} / \mathrm{L}$ \\
\hline & & & 1 & $\begin{array}{l}81 \\
49\end{array}$ & 1000 & u & & $\mathrm{ug} / \mathrm{L}$ \\
\hline & & & $\begin{array}{l}1 \\
1\end{array}$ & 120 & 1000 & u & & ug/L \\
\hline & & & 1 & 220 & 500 & u & & $\mathrm{ug} / \mathrm{L}$ \\
\hline & & & 1 & 66 & 500 & $u$ & & $\mathrm{ug} / \mathrm{L}$ \\
\hline & & & 1 & 55 & 500 & u & & $\mathrm{ug} / \mathrm{L}$ \\
\hline & & & 1 & 100 & 500 & u & & ug/L \\
\hline & & & 1 & 67 & 500 & u & & $u g / L$ \\
\hline & & & 1 & 110 & 1000 & u & & $u g / L$ \\
\hline & & & 1 & 100 & 1000 & u & & ug/L \\
\hline & & & 1 & 91 & 500 & u & & ug/L \\
\hline & & & 1 & 100 & 500 & u & & $\mathrm{ug} / \mathrm{L}$ \\
\hline & & & 1 & 40 & 1000 & u & & $u g / L$ \\
\hline & & & 1 & 52 & 500 & $u$ & & $u g / L$ \\
\hline & & & 1 & 61 & 1000 & $u$ & & ug/L \\
\hline & & & 1 & 120 & 1000 & u & & $\mathrm{ug} / \mathrm{L}$ \\
\hline & & & 1 & 57 & 500 & $u$ & & $u g / L$ \\
\hline & & & 1 & 45 & 500 & u & & ug/L \\
\hline & & & 1 & 110 & 500 & $u$ & & $u g / L$ \\
\hline & & & 1 & 150 & 500 & u & & ug $/ \mathrm{L}$ \\
\hline & & & 1 & 150 & 500 & $\mathrm{u}$ & & $\mathrm{ug} / \mathrm{L}$ \\
\hline & & & 1 & 190 & 1000 & u & & $\mathrm{ug} / \mathrm{L}$ \\
\hline & & & 1 & 53 & 500 & U & & $\mathrm{ug} / \mathrm{L}$ \\
\hline & & & 1 & 140 & 500 & $\mathrm{u}$ & & $\mathrm{ug} / \mathrm{L}$ \\
\hline & & & 1 & 70 & 500 & u & & $u g / L$ \\
\hline & & & 1 & 120 & 500 & $u$ & & $\mathrm{ug} / \mathrm{L}$ \\
\hline & & & 1 & 120 & 500 & $u$ & & $\mathrm{ug} / \mathrm{L}$ \\
\hline & & & 1 & 61 & 500 & u & & $u g / L$ \\
\hline & & & 1 & 97 & 500 & $u$ & & ug/L \\
\hline & & & 1 & 69 & 500 & $u$ & & $\mathrm{ug} / \mathrm{L}$ \\
\hline & & & 1 & 140 & 500 & u & & $\mathrm{ug} / \mathrm{L}$ \\
\hline & & & 1 & 160 & 500 & U & & $\mathrm{ug} / \mathrm{L}$ \\
\hline
\end{tabular}

UNCLASSIFIED 
UNCLASSIFIED

LIMS 03.08 .048

03/12/2010 16:03

\begin{tabular}{|c|c|}
\hline 132649 & Dibenzofuran \\
\hline 84662 & Diethylphthalate \\
\hline 131113 & Dimethylphthalate \\
\hline 206440 & Fluoranthene \\
\hline 86737 & Fluorene \\
\hline 118741 & Hexachlorobenzene \\
\hline 77474 & Hexachlorocyclopentadiene \\
\hline 67721 & Hexachloroethane \\
\hline 193395 & Indeno $(1,2,3$-cd) pyrene \\
\hline 78591 & Isophorone \\
\hline 621647 & $\mathrm{~N}$-Nitroso-di-n-propylamine \\
\hline 62759 & $\mathrm{~N}-\mathrm{Ni}$ trosodime thylamine \\
\hline 86306 & N-Nitrosodiphenylamine \\
\hline 91203 & $\begin{array}{l}\text { Naphthalene } \\
\text { Nitrobenzene }\end{array}$ \\
\hline $\begin{array}{l}98953 \\
87865\end{array}$ & $\begin{array}{l}\text { Nitrobenzene } \\
\text { Pentachlorophenol }\end{array}$ \\
\hline 85018 & Phenanthrene \\
\hline 108952 & Phenol \\
\hline 129000 & Pyrene \\
\hline TIC Id & TIC Name \\
\hline$-\cdots$ & 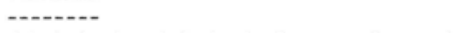 \\
\hline 000000000 & (E)-3,6-Dimethy $1-6$-vinylocta-1,3,7-trien \\
\hline 000000000 & $\begin{array}{l}2,4-D I N I T R O P H E N O L-D 3 \\
\text { unknown }\end{array}$ \\
\hline 001794861 & Phosgene oxime $\$ \$$ Phosgenox $\$ \$ C x \$ \$ \mathrm{Hyd}$ \\
\hline 002562370 & Cyclohexene, 1-nitro- $\$ \$ 1$-Nitrocyclohex \\
\hline 017065188 & Bicyclo[2.1.0]pentane, 1,4-dimethyl- $\$ \$$ \\
\hline 000123422 & 2-Pentanone, 4-hydroxy-4-methyl- (CAS) \$ \\
\hline 000000000 & 2-NITROPHENOL-D4 \\
\hline 001526176 & 2-Fluoro-6-nitrophenol \$\$ Phenol, 2-fluo \\
\hline 001561860 & 2-Chlorocyclohexanol \\
\hline & $\begin{array}{l}\text { unknown } \\
\text { unknown }\end{array}$ \\
\hline & unknown \\
\hline & unknown \\
\hline & unknown \\
\hline & unknown \\
\hline & unknown \\
\hline & unknown \\
\hline & unknown \\
\hline & unknown \\
\hline
\end{tabular}

Analytical Chemistry Organization (Quality Services)

Page 2 of 2

P.O. Box 2009 oak Ridge, TN 378318244 (865) 574-2284

Lab Smpl Id: A100550084 official Report

Cust Smpl Id: 300 Mrad Hanford Sim

\begin{tabular}{|c|c|c|c|c|c|}
\hline 1 & 92 & 500 & u & & $\mathrm{ug} / \mathrm{L}$ \\
\hline 1 & 87 & 500 & u & & $\mathrm{ug} / \mathrm{L}$ \\
\hline 1 & 100 & 500 & u & & $\mathrm{ug} / \mathrm{L}$ \\
\hline 1 & 51 & 500 & u & & $\mathrm{ug} / \mathrm{L}$ \\
\hline 1 & 74 & 500 & u & & $u g / L$ \\
\hline 1 & 66 & 500 & u & & $\mathrm{ug} / \mathrm{L}$ \\
\hline 1 & 260 & 500 & u & & $\mathrm{ug} / \mathrm{L}$ \\
\hline 1 & 150 & 500 & u & & $\mathrm{ug} / \mathrm{L}$ \\
\hline 1 & 100 & 500 & u & & $\mathrm{ug} / \mathrm{L}$ \\
\hline 1 & 70 & 500 & u & & $\mathrm{ug} / \mathrm{L}$ \\
\hline 1 & 65 & 500 & u & & $\mathrm{ug} / \mathrm{L}$ \\
\hline 1 & 150 & 500 & u & & $u g / L$ \\
\hline 1 & 59 & 500 & u & & $\mathrm{ug} / \mathrm{L}$ \\
\hline 1 & 97 & 500 & u & & $\mathrm{ug} / \mathrm{L}$ \\
\hline 1 & 48 & 500 & u & & $\mathrm{ug} / \mathrm{L}$ \\
\hline 1 & 54 & 1000 & u & & $\mathrm{ug} / \mathrm{L}$ \\
\hline 1 & 62 & 500 & u & & $u g / L$ \\
\hline 1 & 92 & 500 & $u$ & & $\mathrm{ug} / \mathrm{L}$ \\
\hline \multirow[t]{24}{*}{1} & 110 & 500 & $u$ & & $\mathrm{ug} / \mathrm{L}$ \\
\hline & Retention & & Estimated & EPA & \\
\hline & Time & Unit & Conc & Qua 7 & Unit \\
\hline & --- & --- & - - & $\cdots$ & $\ldots$ \\
\hline & 12.81 & $\min$ & 1700 & JN & $\mathrm{ug} / \mathrm{L}$ \\
\hline & 19.15 & $\min$ & 1400 & JN & $u g / L$ \\
\hline & 11.18 & $\min$ & 1100 & J & $\mathrm{ug} / \mathrm{L}$ \\
\hline & 8.35 & $\min$ & 2300 & JN & $u g / L$ \\
\hline & 14.07 & $\min$ & 8100 & JN & $\mathrm{ug} / \mathrm{L}$ \\
\hline & 15.57 & $\min$ & 920 & JN & $\mathrm{ug} / \mathrm{L}$ \\
\hline & 7.47 & $\min$ & 4700 & JN & $\mathrm{ug} / \mathrm{L}$ \\
\hline & 13.05 & $\min$ & 2000 & JN & $\mathrm{ug} / \mathrm{L}$ \\
\hline & 13.44 & $\min$ & 1100 & JN & $\mathrm{ug} / \mathrm{L}$ \\
\hline & 11.05 & $\min$ & 1200 & JN & $\mathrm{ug} / \mathrm{L}$ \\
\hline & 12.45 & $\min$ & 950 & 3 & $u g / L$ \\
\hline & 12.68 & $\min$ & 3700 & J & $u g / L$ \\
\hline & 14.77 & $\min$ & 5300 & J & $u g / L$ \\
\hline & 15.34 & $\min$ & 5900 & J & $\mathrm{ug} / \mathrm{L}$ \\
\hline & 16.35 & $\min$ & 4300 & J & $\mathrm{ug} / \mathrm{L}$ \\
\hline & 16.44 & $\min$ & 1200 & J & $\mathrm{ug} / \mathrm{L}$ \\
\hline & 17.63 & $\min$ & 5200 & J & $\mathrm{ug} / \mathrm{L}$ \\
\hline & 18.24 & $\min$ & 1900 & J & $\mathrm{ug} / \mathrm{L}$ \\
\hline & 18.66 & $\min$ & 1200 & 3 & $\mathrm{ug} / \mathrm{L}$ \\
\hline & 6.81 & $\min$ & 20000 & J & ug/L \\
\hline
\end{tabular}

Sample Test Comments: Target Compound(s) Initial Calibration exceeding 15\% RSD:

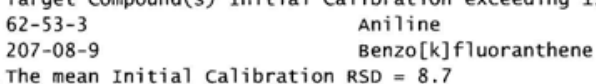

The mean Initial Calibration RSD $=8.7$

Comment objects:

EPA Qualifiers:

J - Estimated value.

N - Presumptive evidence of a compound. (GC/MS flag)

$U$ - Analyte analyzed for but undetected. Analyte result was below the contract required quantitation limit (CRQL).

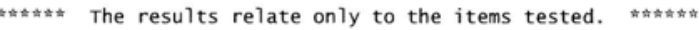

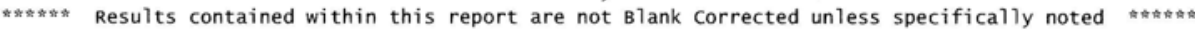

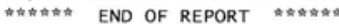

UNCLASSIFIED 
UNCLASSIFIED

LIMS 03.08 .048

03/12/2010 16:02

Analytical Chemistry organization (Quality Services)

Page 1 of 2

P.0. Box 2009 oak Ridge, TN 378318244 (865) 574-2284

Lab Smp 1 Id: A100550083

official Report

Cust Smpl Id: 250 Mrad Hanford Sim

Project: L MS MPO SCHUH SVOA

Proj Mgr: L P BURNETT (865) 576-9141

Customer: L P BURNETT Bldg 9995 MS-8189 (865) 576-9141

\begin{tabular}{|c|c|c|c|c|c|c|c|}
\hline Matrix & $\begin{array}{l}\text { Chain of } \\
\text { Custody }\end{array}$ & $\begin{array}{l}\text { Work Agreement } \\
\text { Number }\end{array}$ & $\begin{array}{l}\text { Charge } \\
\text { Number }\end{array}$ & $\begin{array}{l}\text { Date } \\
\text { Sampled }\end{array}$ & $\begin{array}{l}\text { Date } \\
\text { Received }\end{array}$ & $\begin{array}{l}\text { Date } \\
\text { Needed }\end{array}$ & $\begin{array}{l}\text { Date } \\
\text { Approved }\end{array}$ \\
\hline -...... & --no- & --on- & -...-- & --- & - & -.... & - \\
\hline LIQUID & 117936 & 81473 & $745 A 0221$ & 02/23/2010 00:00 & $02 / 23 / 2010 \quad 14: 30$ & 03/09/2010 23:59 & $03 / 12 / 2010$ \\
\hline
\end{tabular}

Customer comments:

Lab Comments:

Comment objects:

$\ll<<$ Organic $\gg \gg>$

Test: SVOA8270 (Semi-Volatile Organics by GC/MS) Basis: $A R$ Test Req Cnt: 01

Analysis Method: SW846 8270C (Y50-AC-65-7316)

Approver: J T HOFFARTH/Data Approver

QC Batch/File: QC10070010/5314/53787

Prep Method/Date: SW846 3580A 02/24/2010 13:00 Test Status: APPROVED

HT Deadline: 04/05/2010 23:59

Date Analyzed: $03 / 10 / 201018: 33$

Lab Group: OYGCMS

\begin{tabular}{|c|c|}
\hline Analyte Id & Analyte Name \\
\hline 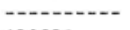 & - \\
\hline 120821 & 1,2,4-Trichlorobenzene \\
\hline 95501 & 1,2-Dichlorobenzene \\
\hline 122667 & 1,2-Diphenylhydrazine \\
\hline 541731 & 1,3-Dichlorobenzene \\
\hline 106467 & 1,4-Dichlorobenzene \\
\hline 95954 & 2,4,5-Trichlorophenol \\
\hline 88062 & 2,4,6-Trichlorophenol \\
\hline 120832 & 2,4-Dich1orophenol \\
\hline 105679 & 2,4-Dimethy 7 pheno 1 \\
\hline 51285 & 2,4-Dinitrophenol \\
\hline 121142 & 2,4-Dinit rotoluene \\
\hline 606202 & 2,6-Dinitrotoluene \\
\hline 91587 & 2-Chloronaphthalene \\
\hline 95578 & 2-Chlorophenol \\
\hline 534521 & 2-Methy1-4,6-dinitrophenol \\
\hline 91576 & 2-Methylnaphthalene \\
\hline 95487 & 2-Methylphenol \\
\hline 88744 & 2-Nit robenzenamine \\
\hline 88755 & 2-Nitrophenol \\
\hline 91941 & 3,3'-Dich Torobenzidine \\
\hline N2799 & 3- and 4- Methylphenol \\
\hline 99092 & 3-Nitrobenzenamine \\
\hline 101553 & 4-Bromopheny 1 pheny 1 ether \\
\hline 59507 & 4-Ch1oro-3-methy 1 pheno 1 \\
\hline 106478 & 4-Chlorobenzenamine \\
\hline 7005723 & 4-Ch 1 oropheny 1 pheny 1 ether \\
\hline 100016 & 4-Nitrobenzenamine \\
\hline 100027 & 4-Nitrophenol \\
\hline 83329 & Acenaphthene \\
\hline 208968 & Acenaphthylene \\
\hline 62533 & Aniline \\
\hline 120127 & Anthracene \\
\hline 100516 & Benzenemethanol \\
\hline 92875 & Benzidine \\
\hline 56553 & Benzo(a) anthracene \\
\hline 50328 & Benzo(a)pyrene \\
\hline 205992 & Benzo(b)fluoranthene \\
\hline 191242 & Benzo(ghi)perylene \\
\hline 207089 & Benzo $(k)$ fluoranthene \\
\hline 65850 & Benzoic acid \\
\hline 111911 & Bis (2-chloroethoxy)methane \\
\hline 111444 & Bis(2-ch1oroethy 1$)$ ether \\
\hline 108601 & Bis(2-ch1oroisopropy 1 ) ether \\
\hline 117817 & Bis(2-ethylhexy1)phthalate \\
\hline 85687 & Butylbenzylphthalate \\
\hline 86748 & Carbazole \\
\hline 218019 & Chrysene \\
\hline 84742 & Di-n-butylphthalate \\
\hline 117840 & Di-n-octylphthalate \\
\hline 53703 & Dibenz $(\mathrm{a}, \mathrm{h})$ anthracene \\
\hline
\end{tabular}

\begin{tabular}{|c|c|c|c|c|c|c|c|c|}
\hline & istome & & Dilution & Lower & & EPA & & \\
\hline HT & Lim & $\mathrm{Fn}$ & Factor & Limit & Result & Qual & Confidence & Unit \\
\hline- & -- & -- & ----- & ----- & ----- & --- & --n-s- & --- \\
\hline & & & 1 & 99 & 500 & u & & $u g / L$ \\
\hline & & & 1 & 110 & 500 & u & & $\mathrm{ug} / \mathrm{L}$ \\
\hline & & & 1 & 59 & 500 & u & & $\mathrm{ug} / \mathrm{L}$ \\
\hline & & & 1 & 170 & 500 & u & & $\mathrm{ug} / \mathrm{L}$ \\
\hline & & & 1 & 180 & 500 & u & & $\mathrm{ug} / \mathrm{L}$ \\
\hline & & & 1 & 190 & 500 & u & & $\mathrm{ug} / \mathrm{L}$ \\
\hline & & & 1 & 100 & 500 & u & & ug/L \\
\hline & & & 1 & 98 & 500 & u & & $\mathrm{ug} / \mathrm{L}$ \\
\hline & & & 1 & 99 & 500 & $u$ & & $u g / L$ \\
\hline & & & 1 & 140 & 1000 & u & & $\mathrm{ug} / \mathrm{L}$ \\
\hline & & & 1 & 130 & 500 & u & & $\mathrm{ug} / \mathrm{L}$ \\
\hline & & & 1 & 97 & 500 & u & & $u g / L$ \\
\hline & & & 1 & 87 & 500 & u & & $u g / L$ \\
\hline & & & 1 & 100 & 500 & $u$ & & $u g / L$ \\
\hline & & & 1 & 110 & 1000 & u & & $\mathrm{ug} / \mathrm{L}$ \\
\hline & & & 1 & 66 & 500 & u & & ug/L \\
\hline & & & 1 & 98 & 500 & u & & $u g / L$ \\
\hline & & & 1 & 110 & 500 & $u$ & & $\mathrm{ug} / \mathrm{L}$ \\
\hline & & & 1 & 81 & 500 & u & & $\mathrm{ug} / \mathrm{L}$ \\
\hline & & & 1 & 49 & 1000 & u & & $\mathrm{ug} / \mathrm{L}$ \\
\hline & & & 1 & 120 & 1000 & $u$ & & $\mathrm{ug} / \mathrm{L}$ \\
\hline & & & 1 & 220 & 500 & u & & $\mathrm{ug} / \mathrm{L}$ \\
\hline & & & 1 & 66 & 500 & u & & $\mathrm{ug} / \mathrm{L}$ \\
\hline & & & 1 & 55 & 500 & u & & $\mathrm{ug} / \mathrm{L}$ \\
\hline & & & 1 & 100 & 500 & $u$ & & $u g / L$ \\
\hline & & & 1 & 67 & 500 & u & & $u g / L$ \\
\hline & & & 1 & 110 & 1000 & u & & $\mathrm{ug} / \mathrm{L}$ \\
\hline & & & 1 & 100 & 1000 & $u$ & & $u g / L$ \\
\hline & & & 1 & 91 & 500 & $u$ & & $\mathrm{ug} / \mathrm{L}$ \\
\hline & & & 1 & 100 & 500 & u & & $u g / L$ \\
\hline & & & 1 & 40 & 1000 & u & & $\mathrm{ug} / \mathrm{L}$ \\
\hline & & & 1 & 52 & 500 & u & & $\mathrm{ug} / \mathrm{L}$ \\
\hline & & & 1 & 61 & 1000 & u & & $u g / L$ \\
\hline & & & 1 & 120 & 1000 & $u$ & & $u g / L$ \\
\hline & & & 1 & 57 & 500 & u & & $u g / L$ \\
\hline & & & 1 & 45 & 500 & u & & $\mathrm{ug} / \mathrm{L}$ \\
\hline & & & 1 & 110 & 500 & u & & $u g / L$ \\
\hline & & & 1 & 150 & 500 & u & & $\mathrm{ug} / \mathrm{L}$ \\
\hline & & & 1 & 150 & 500 & u & & $u g / L$ \\
\hline & & & 1 & 190 & 1000 & $u$ & & $u g / L$ \\
\hline & & & 1 & 53 & 500 & $u$ & & $\mathrm{ug} / \mathrm{L}$ \\
\hline & & & 1 & 140 & 500 & u & & $\mathrm{ug} / \mathrm{L}$ \\
\hline & & & 1 & 70 & 500 & 0 & & $\mathrm{ug} / \mathrm{L}$ \\
\hline & & & 1 & 120 & 500 & $u$ & & $u g / L$ \\
\hline & & & 1 & 120 & 500 & u & & ug/L \\
\hline & & & 1 & 61 & 500 & u & & $u g / L$ \\
\hline & & & 1 & 97 & 500 & $u$ & & $u g / L$ \\
\hline & & & 1 & 69 & 500 & U & & $u g / L$ \\
\hline & & & 1 & 140 & 500 & U & & $\mathrm{ug} / \mathrm{L}$ \\
\hline & & & 1 & 160 & 500 & 0 & & $\mathrm{ug} / \mathrm{L}$ \\
\hline
\end{tabular}

UNCLASSIFIED 
UNCLASSIFIED

LIMS 03.08 .048

03/12/2010 16:02

\begin{tabular}{|c|c|}
\hline 132649 & Dibenzofuran \\
\hline 84662 & Diethylphthalate \\
\hline 131113 & Dimethylphthalate \\
\hline 206440 & Fluoranthene \\
\hline 86737 & Fluorene \\
\hline 118741 & Hexachlorobenzene \\
\hline 77474 & Hexachlorocyclopentadiene \\
\hline 67721 & Hexachloroethane \\
\hline 193395 & Indeno $(1,2,3-c d)$ pyrene \\
\hline 78591 & Isophorone \\
\hline 621647 & $\mathrm{~N}$-Nitroso-di-n-propylamine \\
\hline 62759 & $\mathrm{~N}$-Nit rosodimethylamine \\
\hline 86306 & $\mathrm{~N}$-Ni trosodiphenyl amine \\
\hline 91203 & Naphthalene \\
\hline 98953 & Nitrobenzene \\
\hline 87865 & Pentachlorophenol \\
\hline 85018 & Phenanthrene \\
\hline 108952 & Phenol \\
\hline 129000 & Pyrene \\
\hline
\end{tabular}

TIC Id TIC Name

001561860 2-Chlorocyclohexanol \$\$ Cyclohexanol, 2-

001526176 2-Fluoro-6-nitrophenol \$s Phenol, 2-fluo

000000000 2-NITROPHENOL-D4

000123422 2-Pentanone, 4-hydroxy-4-methyl- (CAS) S

002562370 cyclohexene, 1-nitro- \$\$ 1-Nitrocyclohex

074752946 Cyclopropane, 1-chloro-2-(1-propeny 1)-

001794861 phosgene oxime \$S Phosgenox \$S CX \$S Hyd

unknown

unknown

unknown

unknown

unknown

unknown

unknown

unknown

unknown

unknown

unknown

unknown
Analytical Chemistry organization (Quality Services)

P.o. Box 2009 oak Ridge, TN 378318244 (865) 574-2284

Page 2 of 2 official Report

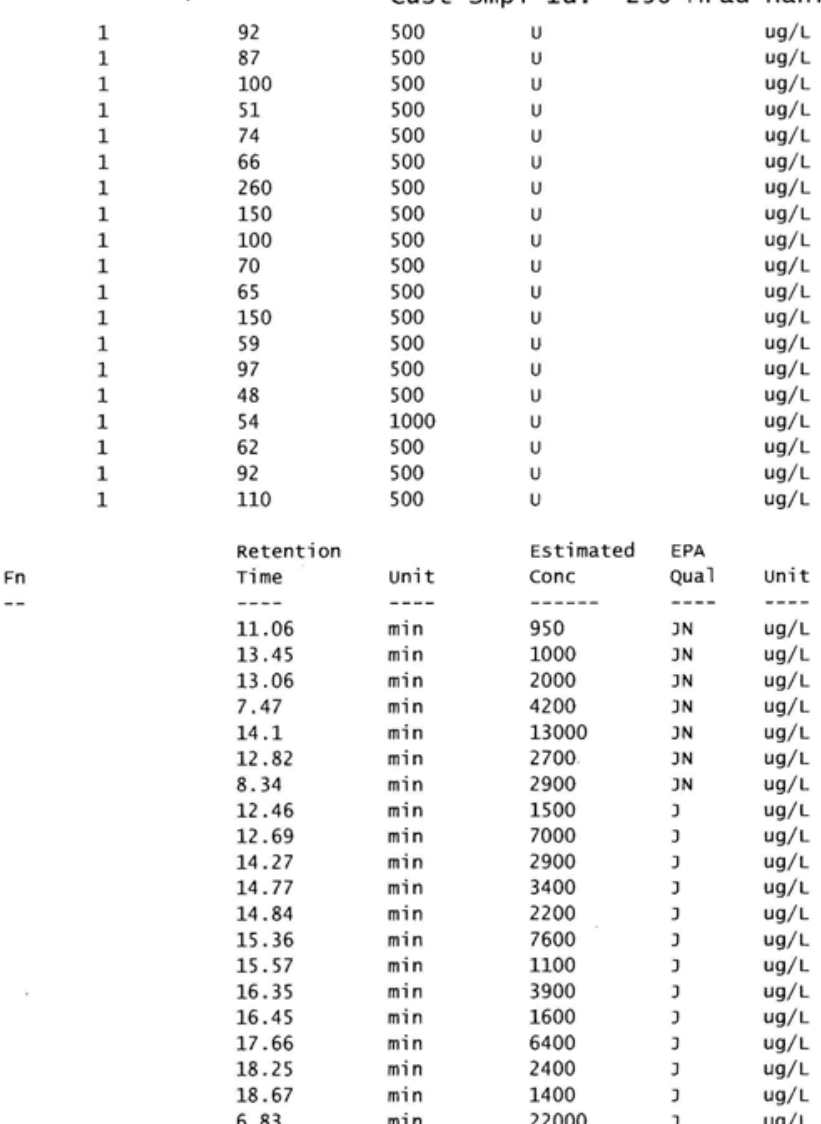

Sample Test Comments: Target Compound(s) Initial Calibration exceeding 15\% RSD:

Comment objects:

EPA Qualifiers:

J - Estimated value.

$\mathrm{N}$ - Presumptive evidence of a compound. (GC/MS flag)

$U$ - Analyte analyzed for but undetected. Analyte result was below the contract required quantitation limit (CRQL).
Lab Smpl Id: A100550083 $\mathrm{ug} / \mathrm{L}$

ug/L

$u g / L$

ug/L

$u g / L$

ug/L

ug/L

ug/L

g/L

---

ug/L

$u g / L$

ug/L

ug/L.

ug/L

$u g / L$

ug/L

$\mathrm{ug} / \mathrm{L}$

ug/L

$\mathrm{ug} / \mathrm{L}$

$u g / L$

ug/L

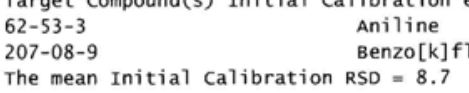 \\ 207-08-9 Benzo[k]fluoranthene}


UNCLASSIFIED

LIMS 03.08 .048

$04 / 12 / 2010 \quad 15: 45$

Analytical Chemistry organization (Quality Services)

Page 1 of 2

P.o. Box 2009 oak Ridge, TN 378318244 (865) 574-2284

A100690180

Project: L MS MPO SCHUH SVOA

official Report

Cust Smpl Id: HANDORD 200 MRAD

Proj Mgr: L P BURNETT (865) 576-9141

Customer: L P BURNETT Bldg 9995 MS-8189 (865) 576-9141

\begin{tabular}{|c|c|c|c|c|c|c|c|}
\hline Matrix & $\begin{array}{l}\text { Chain of } \\
\text { Custody }\end{array}$ & $\begin{array}{l}\text { Work Agreement } \\
\text { Number }\end{array}$ & $\begin{array}{l}\text { Charge } \\
\text { Number }\end{array}$ & $\begin{array}{l}\text { Date } \\
\text { Sampled }\end{array}$ & $\begin{array}{l}\text { Date } \\
\text { Received }\end{array}$ & $\begin{array}{l}\text { Date } \\
\text { Needed }\end{array}$ & $\begin{array}{l}\text { Date } \\
\text { Approved }\end{array}$ \\
\hline---- & ------ & ----- & -..-- & - & - & $-\cdots--$ & n-n- \\
\hline LIQUID & 117945 & 81473 & $745 A 0221$ & 03/09/2010 00:00 & $03 / 09 / 2010 \quad 14: 10$ & $03 / 23 / 2010 \quad 23: 59$ & $04 / 12 / 2010 \quad 15: 45$ \\
\hline
\end{tabular}

Sample Description: $2 \mathrm{M} \mathrm{NaOH}$

Sample Status: APPROVED

Location:

Sample Approver: J T HOFFARTH/Data Approver

Customer comments:

Lab Comments:

Comment objects:

$\ll<<$ Organic $\gg \gg>$

Test: SVOA8270 (Semi-Volatile Organics by GC/MS)

Rpt Basis: AR Test Req Cnt: 01

Analysis Method: SW846 8270C (Y50-AC-65-7316)

Approver: ] T HOFFARTH/Data Approver

QC Batch/File: QC10102020/5332/53810
Prep Method/Date: SW846 3580A 03/16/2010 15:00 Test Status: APPROVED

HT Deadline: 04/25/2010 23:59

Date Analyzed: 03/30/2010 16:21

Lab Group: OYGCMS

$\begin{array}{ll}\text { Analyte Id } & \text { Analyte Name } \\ --20821 & \text { 1,2,4-Trichlorobenzene } \\ 95501 & \text { 1,2-Dichlorobenzene } \\ 122667 & \text { 1,2-Diphenylhydrazine } \\ 541731 & \text { 1,3-Dichlorobenzene } \\ 106467 & \text { 1,4-Dichlorobenzene } \\ 95954 & \text { 2,4,5-Trichlorophenol } \\ 88062 & \text { 2,4,6-Trichlorophenol } \\ 120832 & \text { 2,4-Dichlorophenol } \\ 105679 & \text { 2,4-Dimethylphenol } \\ 51285 & \text { 2,4-Dinitrophenol } \\ 121142 & \text { 2,4-Dinitrotoluene } \\ 606202 & \text { 2,6-Dinitrotoluene } \\ 91587 & \text { 2-Chloronaphthalene } \\ 95578 & \text { 2-Chlorophenol } \\ 534521 & \text { 2-Methyl-4,6-dinitrophenol } \\ 91576 & \text { 2-Methylnaphthalene } \\ 95487 & \text { 2-Methylphenol } \\ 88744 & \text { 2-Nitrobenzenamine } \\ 88755 & \text { 2-Nitrophenol } \\ 91941 & \text { 3,3'-Dichlorobenzidine } \\ \text { N2799 } & \text { 3- and 4- Methylphenol } \\ 99092 & \text { 3-Nitrobenzenamine } \\ 101553 & \text { 4-Bromophenyl phenyl ether } \\ 59507 & \text { 4-Chloro-3-methylphenol } \\ 106478 & \text { 4-Chlorobenzenamine } \\ 7005723 & \text { 4-Chlorophenylpheny1 ether } \\ 100016 & \text { 4-Nitrobenzenamine } \\ 100027 & \text { 4-Nitrophenol } \\ 83329 & \text { Acenaphthene } \\ 208968 & \text { Acenaphthylene } \\ 62533 & \text { Aniline } \\ 120127 & \text { Anthracene } \\ 100516 & \text { Benzenemethanol } \\ 92875 & \text { Benzidine } \\ 56553 & \text { Benzo(a)anthracene } \\ 50328 & \text { Benzo(a)pyrene } \\ 205992 & \text { Benzo(b)fluoranthene } \\ 191242 & \text { Benzo(ghi)perylene } \\ 207089 & \text { Benzo(k)fluoranthene } \\ 65850 & \text { Benzoic acid } \\ 111911 & \text { Bis(2-chloroethoxy)methane } \\ 111444 & \text { Bis(2-chloroethyl) ether } \\ 108601 & \text { Bis(2-chloroisopropy1) ether } \\ 117817 & \text { Bis(2-ethylhexy1)phthalate } \\ 85687 & \text { Butylbenzylphthalate } \\ 86748 & \text { Carbazole } \\ 218019 & \text { Chrysene } \\ 117840 & \text { Di-n-butylphthalate } \\ 53703 & \text { Di-n-octylphthalate } \\ & \text { Dibenz(a,h)anthracene } \\ & \end{array}$

\begin{tabular}{|c|c|c|c|c|c|c|c|c|}
\hline \multicolumn{3}{|c|}{ Customer } & \multirow{2}{*}{$\begin{array}{l}\text { Dilution } \\
\text { Factor }\end{array}$} & \multirow{2}{*}{$\begin{array}{l}\text { Lower } \\
\text { Limit }\end{array}$} & \multirow[b]{2}{*}{ Result } & \multirow{2}{*}{$\begin{array}{l}\text { EPA } \\
\text { Qual }\end{array}$} & \multirow[b]{2}{*}{ Confidence } & \multirow[b]{2}{*}{ Unit } \\
\hline HT & Lim & $\mathrm{Fn}$ & & & & & & \\
\hline-- & --- & -- & $-\cdots$ & $-\cdots$ & --n- & $\cdots$ & - & --- \\
\hline & & & 1 & 99 & 500 & u & & $u g / L$ \\
\hline & & & 1 & 110 & 500 & u & & $u g / L$ \\
\hline & & & 1 & 59 & 500 & u & & $u g / L$ \\
\hline & & & 1 & 170 & 500 & u & & $u g / L$ \\
\hline & & & 1 & 180 & 500 & u & & $\mathrm{ug} / \mathrm{L}$ \\
\hline & & & 1 & 190 & 500 & u & & $u g / L$ \\
\hline & & & 1 & 100 & 500 & u & & $\mathrm{ug} / \mathrm{L}$ \\
\hline & & & 1 & 98 & 500 & u & & $u g / L$ \\
\hline & & & 1 & 99 & 500 & u & & $\mathrm{ug} / \mathrm{L}$ \\
\hline & & & 1 & 140 & 1000 & u & & $\mathrm{ug} / \mathrm{L}$ \\
\hline & & & 1 & 130 & 500 & u & & $\mathrm{ug} / \mathrm{L}$ \\
\hline & & & 1 & 97 & 500 & u & & $u g / L$ \\
\hline & & & 1 & 87 & 500 & u & & $\mathrm{ug} / \mathrm{L}$ \\
\hline & & & 1 & 100 & 500 & u & & $u g / L$ \\
\hline & & & 1 & 110 & 1000 & u & & ug $/ \mathrm{L}$ \\
\hline & & & 1 & 66 & 500 & U & & ug/L \\
\hline & & & 1 & 98 & 500 & U & & ug $/ \mathrm{L}$ \\
\hline & & & 1 & 110 & 500 & u & & $u g / L$ \\
\hline & & & 1 & 81 & 500 & u & & $\mathrm{ug} / \mathrm{L}$ \\
\hline & & & 1 & 49 & 1000 & u & & $u g / L$ \\
\hline & & & 1 & 120 & 1000 & u & & $\mathrm{ug} / \mathrm{L}$ \\
\hline & & & 1 & 220 & 500 & u & & $\mathrm{ug} / \mathrm{L}$ \\
\hline & & & 1 & 66 & 500 & u & & $\mathrm{ug} / \mathrm{L}$ \\
\hline & & & 1 & 55 & 500 & u & & $u g / L$ \\
\hline & & & 1 & 100 & 500 & U & & $\mathrm{ug} / \mathrm{L}$ \\
\hline & & & 1 & 67 & 500 & u & & $\mathrm{ug} / \mathrm{L}$ \\
\hline & & & 1 & 110 & 1000 & u & & $\mathrm{ug} / \mathrm{L}$ \\
\hline & & & 1 & 100 & 1000 & u & & ug/L \\
\hline & & & 1 & 91 & 500 & u & & ug/L \\
\hline & & & 1 & 100 & 500 & u & & $\mathrm{ug} / \mathrm{L}$ \\
\hline & & & 1 & 40 & 1000 & U & & $\mathrm{ug} / \mathrm{L}$ \\
\hline & & & 1 & 52 & 500 & U & & $\mathrm{ug} / \mathrm{L}$ \\
\hline & & & 1 & 61 & 1000 & u & & $\mathrm{ug} / \mathrm{L}$ \\
\hline & & & 1 & 120 & 1000 & u & & $\mathrm{ug} / \mathrm{L}$ \\
\hline & & & 1 & 57 & 500 & u & & $\mathrm{ug} / \mathrm{L}$ \\
\hline & & & 1 & 45 & 500 & u & & ug/L \\
\hline & & & 1 & 110 & 500 & u & & $\mathrm{ug} / \mathrm{L}$ \\
\hline & & & 1 & 150 & 500 & u & & $\mathrm{ug} / \mathrm{L}$ \\
\hline & & & 1 & 150 & 500 & u & & $\mathrm{ug} / \mathrm{L}$ \\
\hline & & & 1 & 190 & 750 & 了 & & $\mathrm{ug} / \mathrm{L}$ \\
\hline & & & 1 & 53 & 500 & u & & $\mathrm{ug} / \mathrm{L}$ \\
\hline & & & 1 & 140 & 500 & u & & $\mathrm{ug} / \mathrm{L}$ \\
\hline & & & 1 & 70 & 500 & u & & $\mathrm{ug} / \mathrm{L}$ \\
\hline & & & 1 & 120 & 500 & u & & $u g / L$ \\
\hline & & & 1 & 120 & 500 & u & & $u g / L$ \\
\hline & & & 1 & 61 & 500 & u & & ug/L \\
\hline & & & 1 & 97 & 500 & u & & $\mathrm{ug} / \mathrm{L}$ \\
\hline & & & 1 & 69 & 500 & u & & $\mathrm{ug} / \mathrm{L}$ \\
\hline & & & 1 & 140 & 500 & u & & $u g / L$ \\
\hline & & & 1 & 160 & 500 & U & & $\mathrm{ug} / \mathrm{L}$ \\
\hline
\end{tabular}

UNCLASSIFIED 
UNCLASSIFIED

LIMS 03.08 .048

$04 / 12 / 201015: 45$

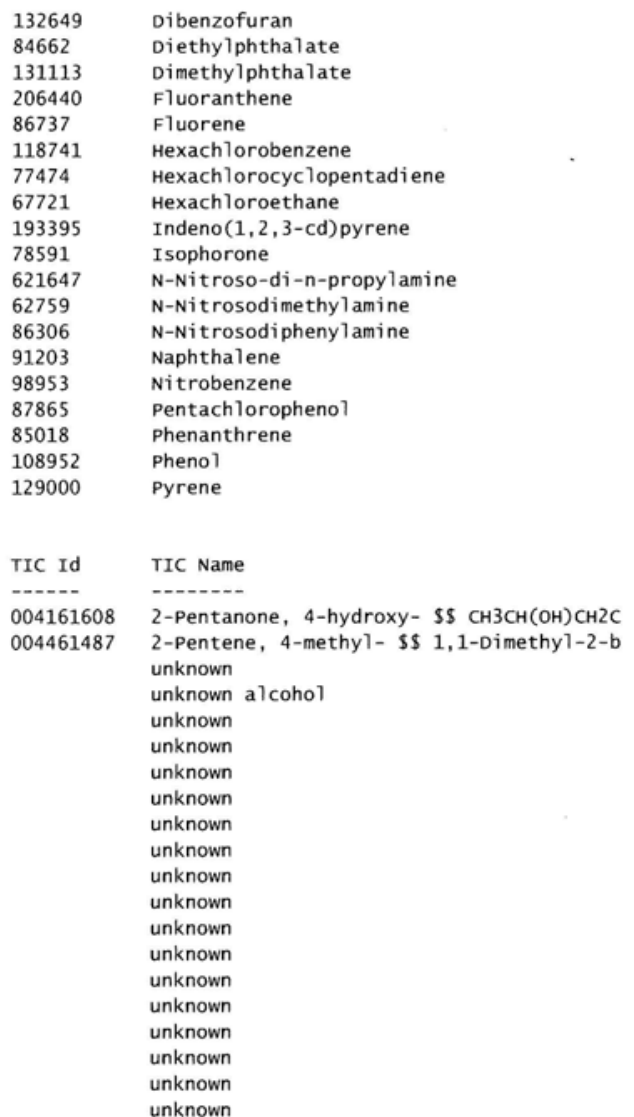

Analytical Chemistry organization (Quality Services) P.O. Box 2009 oak Ridge, TN 378318244 (865) 574-2284

Page 2 of 2

Lab Smp1 Id: A100690180 official Report

Cust Smp1 Id: HANDORD 200 MRAD

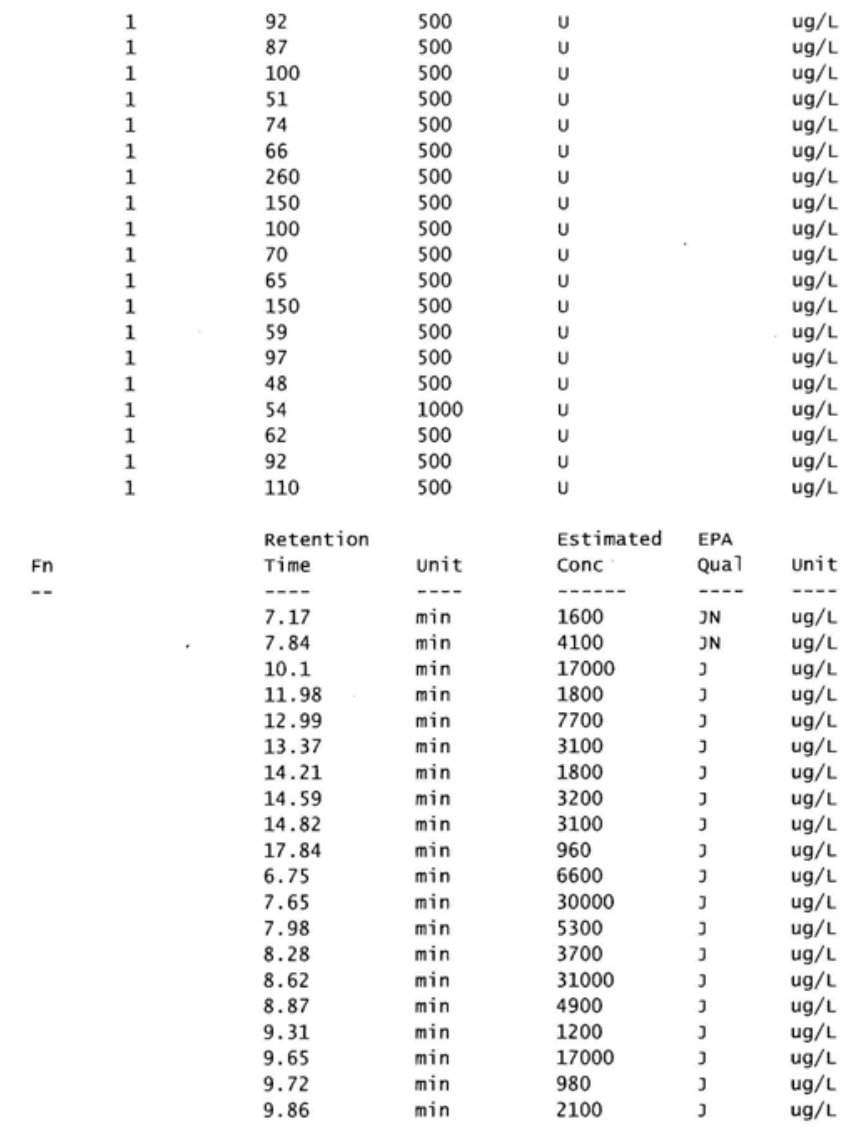

Sample Test Comments: Target compound(s) Initial Calibration exceeding 15\% RSD:

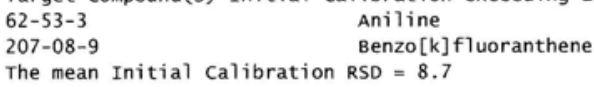

Comment objects:

EPA Qualifiers:

J - Estimated value.

$\mathrm{N}$ - Presumptive evidence of a compound. (GC/MS flag)

U - Analyte analyzed for but undetected. Analyte result was below the contract required quantitation limit (CRQL).

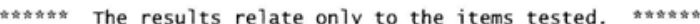

Results contained within this report are not Blank corrected unless specifically noted *n

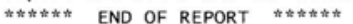

UNCLASSIFIED 
UNCLASSIFIED

LIMS 03.08 .048

12/02/2009 13:44

Project: L MS MPO SCHUH SVOA

Customer: L P BURNETT Bldg 9995 MS-8189 (865) 576-9141

\begin{tabular}{|c|c|c|c|c|c|c|c|}
\hline Matrix & $\begin{array}{l}\text { Chain of } \\
\text { Custody }\end{array}$ & $\begin{array}{l}\text { Work Agreement } \\
\text { Number }\end{array}$ & $\begin{array}{l}\text { Charge } \\
\text { Number }\end{array}$ & $\begin{array}{l}\text { Date } \\
\text { Sampled }\end{array}$ & $\begin{array}{l}\text { Date } \\
\text { Received }\end{array}$ & $\begin{array}{l}\text { Date } \\
\text { Needed }\end{array}$ & $\begin{array}{l}\text { Date } \\
\text { Approved }\end{array}$ \\
\hline$-\cdots-$ & ------ & ----- & ----- & 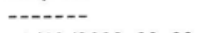 & $----\overline{-}$ & - n- & - n-c- \\
\hline LIQUID & 117883 & 81473 & $745 A 0221$ & $11 / 10 / 200900: 00$ & $11 / 10 / 200915: 30$ & $12 / 09 / 2009 \quad 23: 59$ & $12 / 02 / 2009$ \\
\hline
\end{tabular}

Customer comments:

Lab Comments:

Comment objects:

$\ll<<$ organic $\gg \gg>$

Test: svoA8270 (Semi-volatile organics by GC/MS)
Rpt Basis: AR

Test Req Cnt: 01

Analysis Method: SW846 8270C (Y50-AC-65-7316)

Approver: ] T HOFFARTH/Data Approver

QC Batch/File: QC09336003/5206/53706
Page 1 of 2

Lab Smp1 Id: A093150151
Proj Mgr: L P BURNETT (865) 576-9141

Cust Smp 1 Id: Hanford 150 Mrad 
UNCLASSIFIED

LIMS 03.08.048

$12 / 02 / 200913: 44$

132649 Dibenzofuran

Diethylphthalate

131113 Dimethylphthalate

206440 Fluoranthene

86737 Fluorene

118741 Hexachlorobenzene

77474 Hexachlorocyclopentadiene

67721 Hexachloroethane

193395 Indeno(1,2,3-cd)pyrene

78591 Isophorone

$621647 \quad \mathrm{~N}$-Nitroso-di-n-propylamine

$62759 \quad \mathrm{~N}$-Nitrosodimethylamine

$86306 \quad \mathrm{~N}$-Nitrosodiphenylamine

$91203 \quad$ Naphthalene

$98953 \quad$ Nitrobenzene

87865 Pentachlorophenol

85018 Phenanthrene

108952 Phenol

129000 Pyrene

TIC Id TIC Name

000815474 1-Pentene, 3-bromo-4-methyl-

023542510 1-Pentene, 5-nitro- \$s 5-Nitro-1-pentene unknown alcohol/alkoxy cpd unknown alcohol

unknown

007119893 Methane, dichloronitro- \$\$ Dichloronitro

017165558 Butanoic acid, heptafluoro-, 2-propeny1

000123422 2-Pentanone, 4-hydroxy-4-methyl- (CAS) S

000870633 2-Butene, 1-bromo-3-methyl- (CAS) \$\$ .ga

000870633 2-Butene, 1-bromo-3-methyl- \$\$ .gamma.,.

unknown

unknown alcohol/alkoxy cpd

unknown

unknown

unknown

unknown

unknown

unknown

unknown

unknown
Analytical Chemistry organization (Quality Services)

Page 2 of 2

P.0. Box 2009 oak Ridge, TN 378318244 (865) 574-2284

Lab Smp1 Id: A093150151

official Report

Cust Smpl Id: Hanford $150 \mathrm{Mrad}$

Fn

$\begin{array}{ll}1 & 100 \\ 1 & 160 \\ 1 & 140 \\ 1 & 170 \\ 1 & 130 \\ 1 & 180 \\ 1 & 240 \\ 1 & 80 \\ 1 & 140 \\ 1 & 100 \\ 1 & 210 \\ 1 & 380 \\ 1 & 170 \\ 1 & 100 \\ 1 & 150 \\ 1 & 240 \\ 1 & 68 \\ 1 & 86 \\ 1 & 170\end{array}$

$\begin{array}{lll}100 & 670 \\ 160 & 670 & \\ 140 & 670 & \\ 170 & 670 & \\ 130 & 670 & \\ 180 & 670 & \\ 240 & 670 & \\ 80 & 670 & \\ 140 & 670 & \\ 100 & 670 & \\ 210 & 670 & \\ 380 & 670 & \\ 170 & 670 & \\ 100 & 670 & \\ 150 & 670 & \\ 240 & 1300 & \\ 68 & 670 & \\ 86 & 670 & \\ 170 & 670 & \end{array}$

$u g / \mathrm{L}$

$u g / L$

$u g / L$

$\mathrm{ug} / \mathrm{L}$

$\mathrm{ug} / \mathrm{L}$

$u g / L$

$u g / L$

$u g / L$

$\mathrm{ug} / \mathrm{L}$

$\mathrm{ug} / \mathrm{L}$

ug/L

$u g / L$

$u g / L$

$u g / L$

ug/L

ug/L

$\mathrm{ug} / \mathrm{L}$

$\mathrm{ug} / \mathrm{L}$

Retention

-.--

6.28

9.5
9.4

9.4

10.31

3.99

7.13

5.82

6.94

5.95

11.18

9.55

12.17

13.06

4.88

5

7.65

7.99
8.1

8.2
Unit
min
min
min
min
min
min
min
min
min
min
min
min
min
min
min
min
min
min
$\min$
$\min$
Estimated EPA Conc Qual Unit

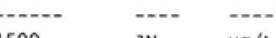

JN ug/L

$1900 \quad$ JN ug/L

$2800 \quad$ J ug/L

3

$u g / L$

$\begin{array}{lll}2500 & \text { JN } & \mathrm{ug} / \mathrm{L} \\ 3000 & \mathrm{JN} & \mathrm{ug} / \mathrm{L}\end{array}$

$15000 \quad J N \quad u g / L$

$15000 \quad J N \quad$ ug/L

$2000 \mathrm{~J} \mathrm{ug} / \mathrm{L}$

$5600 \quad \mathrm{ug} / \mathrm{L}$

$2700 \quad \mathrm{ug} / \mathrm{L}$

$1900 \mathrm{~J} \quad \mathrm{ug} / \mathrm{L}$

$2900 \quad J \quad u g / L$

$1500 \quad \mathrm{ug} / \mathrm{L}$

$7500 \quad J \quad u g / L$

J ug/
JN ug/L

$1200 \quad \mathrm{~J} \quad \mathrm{ug} / \mathrm{L}$

Sample Test comments: Target Compound(s) Initial Calibration exceeding 15\% RSD: 111-44-4 bis(2-Chloroethy1)ether The mean Initial Calibration RSD $=9.3$

Comment objects:

EPA Qualifiers:

J - Estimated value.

N - Presumptive evidence of a compound. (GC/MS flag)

$U$ - Analyte analyzed for but undetected. Analyte result was below the contract required quantitation limit (CRQL).

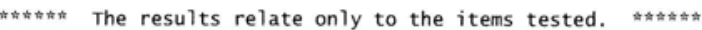

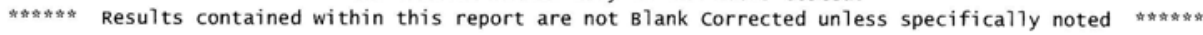

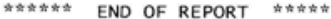

UNCLASSIFIED 


\section{UNCLASSIFIED}

LIMS 03.08 .048

$04 / 12 / 2010 \quad 15: 44$

Project: L MS MPO SCHUH SVOA
Analytical Chemistry organization (Quality Services)

Page 1 of 2

P.0. Box 2009 oak Ridge, TN 378318244 (865) 574-2284

Cust Smp1 Id: H2O 300 NEW

Customer: L P BURNETT B1dg 9995 MS-8189 (865) 576-9141

\begin{tabular}{|c|c|c|c|c|c|c|c|}
\hline Matrix & $\begin{array}{l}\text { Chain of } \\
\text { Custody }\end{array}$ & $\begin{array}{l}\text { work Agreement } \\
\text { Number }\end{array}$ & $\begin{array}{l}\text { Charge } \\
\text { Number }\end{array}$ & $\begin{array}{l}\text { Date } \\
\text { Sampled }\end{array}$ & $\begin{array}{l}\text { Date } \\
\text { Received }\end{array}$ & $\begin{array}{l}\text { Date } \\
\text { Needed }\end{array}$ & $\begin{array}{l}\text { Date } \\
\text { Approved }\end{array}$ \\
\hline---- & $\ldots$ & $-\cdots-$ & $\ldots$ & $-\cdots-$ & - & $-\cdots-$ & - \\
\hline LIQUID & 117945 & 81473 & $745 A 0221$ & 03/09/2010 00:00 & 03/09/2010 $14: 10$ & $03 / 23 / 2010 \quad 23: 59$ & $04 / 12 / 201015: 44$ \\
\hline
\end{tabular}

Sample Description:

Sample status: APPROVED

Location:

Sample Approver: J T HOFFARTH/Data Approver

Customer comments:

Lab comments:

Comment objects:

$\ll<$ Organic $\gg \gg>>$ Test: SVOA8270 (Semi-volatile organics by GC/MS)

Rpt Basis: AR Test Req Cnt: 01

Analysis Method: SW846 8270C (Y50-AC-65-7316)

Approver: J T HOFFARTH/Data Approver

QC Batch/File: QC10102020/5332/53810
Prep Method/Date: Sw846 3580A 03/16/2010 15:00 Test Status: APPROVED

HT Deadline: 04/25/2010 23:59

Date Analyzed: 03/30/2010 15:35 Lab Group: OYGCMS

\begin{tabular}{|c|c|}
\hline Analyte Id & Analyte Name \\
\hline - & - \\
\hline 120821 & 1,2,4-Trichlorobenzene \\
\hline 95501 & 1,2-Dichlorobenzene \\
\hline 122667 & 1,2-Diphenylhydrazine \\
\hline 541731 & 1,3-Dichlorobenzene \\
\hline 106467 & 1,4-Dichlorobenzene \\
\hline 95954 & 2,4,5-Trichlorophenol \\
\hline 88062 & 2,4,6-Trich1orophenol \\
\hline 120832 & 2,4-Dichlorophenol \\
\hline 105679 & 2,4-Dimethy 1 phenol \\
\hline 51285 & 2,4-Dinitrophenol \\
\hline 121142 & 2,4-Dinitrotoluene \\
\hline 606202 & 2,6-Dinitrotoluene \\
\hline 91587 & 2-Chloronaphthalene \\
\hline 95578 & 2-Ch1oropheno 1 \\
\hline 534521 & 2-Methyl-4,6-dinitropheno 1 \\
\hline 91576 & 2-Methylnaphthalene \\
\hline 95487 & 2-Methylphenol \\
\hline 88744 & 2-Nitrobenzenamine \\
\hline 88755 & 2-Nitrophenol \\
\hline 91941 & 3, $3^{\prime}$-Dichlorobenzidine \\
\hline N2799 & 3- and 4- Methylphenol \\
\hline 99092 & 3-Nitrobenzenamine \\
\hline 101553 & 4-8romopheny 1 pheny 1 ether \\
\hline 59507 & 4-Ch1oro-3-methylphenol \\
\hline 106478 & 4-Chlorobenzenamine \\
\hline 7005723 & 4-Ch 1oropheny 1 pheny 1 ether \\
\hline 100016 & 4-Nitrobenzenamine \\
\hline 100027 & 4-Nitrophenol \\
\hline 83329 & Acenaphthene \\
\hline 208968 & Acenaphthylene \\
\hline 62533 & Aniline \\
\hline 120127 & Anthracene \\
\hline 100516 & Benzenemethanol \\
\hline 92875 & Benzidine \\
\hline 56553 & Benzo(a) anthracene \\
\hline 50328 & Benzo(a)pyrene \\
\hline 205992 & Benzo(b)fluoranthene \\
\hline 191242 & Benzo(ghi)perylene \\
\hline 207089 & Benzo(k)fluoranthene \\
\hline 65850 & Benzoic acid \\
\hline 111911 & Bis (2-chloroethoxy)methane \\
\hline 111444 & Bis (2-chloroethyl) ether \\
\hline 108601 & Bis (2-chloroisopropyl) ether \\
\hline 117817 & Bis(2-ethylhexyl)phthalate \\
\hline 85687 & Butylbenzylphthalate \\
\hline 86748 & Carbazole \\
\hline 218019 & Chrysene \\
\hline 84742 & Di-n-butylphthalate \\
\hline 117840 & Di-n-octylphthalate \\
\hline 53703 & Dibenz $(a, h)$ anthracene \\
\hline
\end{tabular}

\begin{tabular}{|c|c|c|c|c|c|c|c|c|}
\hline \multicolumn{3}{|c|}{ Customer } & \multirow{2}{*}{$\begin{array}{l}\text { Dilution } \\
\text { Factor }\end{array}$} & \multirow{2}{*}{$\begin{array}{l}\text { Lower } \\
\text { Limit }\end{array}$} & \multirow[b]{2}{*}{ Result } & EPA & \multirow{2}{*}{ Confidence } & \multirow[b]{2}{*}{ Unit } \\
\hline HT & Lim & Fn & & & & Qual & & \\
\hline-- & -- & -- & - - & $\cdots$ & -...- & $\cdots$ & - & --- \\
\hline & & & 1 & 99 & 500 & u & & $u g / L$ \\
\hline & & & 1 & 110 & 500 & u & & $\mathrm{ug} / \mathrm{L}$ \\
\hline & & & 1 & 59 & 500 & u & & $\mathrm{ug} / \mathrm{L}$ \\
\hline & & & 1 & 170 & 500 & $u$ & & $u g / L$ \\
\hline & & & 1 & 180 & 500 & u & & $u g / L$ \\
\hline & & & 1 & 190 & 500 & $u$ & & $u g / L$ \\
\hline & & & 1 & 100 & 500 & u & & $u g / L$ \\
\hline & & & 1 & 98 & 500 & u & & $u g / L$ \\
\hline & & & 1 & 99 & 500 & u & & $u g / L$ \\
\hline & & & 1 & 140 & 1000 & u & & $\mathrm{ug} / \mathrm{L}$ \\
\hline & & & 1 & 130 & 500 & u & & $u g / L$ \\
\hline & & & 1 & 97 & 500 & u & & ug/L \\
\hline & & & 1 & 87 & 500 & u & & $u g / L$ \\
\hline & & & 1 & 100 & 500 & u & & $u g / L$ \\
\hline & & & 1 & 110 & 1000 & u & & $u g / L$ \\
\hline & & & 1 & 66 & 500 & u & & $u g / L$ \\
\hline & & & 1 & 98 & 500 & u & & $\mathrm{ug} / \mathrm{L}$ \\
\hline & & & 1 & 110 & 500 & $u$ & & $\mathrm{ug} / \mathrm{L}$ \\
\hline & & & 1 & 81 & 500 & $u$ & & $u g / L$ \\
\hline & & & 1 & 49 & 1000 & u & & $\mathrm{ug} / \mathrm{L}$ \\
\hline & & & 1 & 120 & 1000 & u & & $u g / L$ \\
\hline & & & 1 & 220 & 500 & u & & $u g / L$ \\
\hline & & & 1 & 66 & 500 & u & & $u g / L$ \\
\hline & & & 1 & 55 & 500 & u & & $\mathrm{ug} / \mathrm{L}$ \\
\hline & & & 1 & 100 & 500 & u & & $\mathrm{ug} / \mathrm{L}$ \\
\hline & & & 1 & 67 & 500 & $u$ & & $\mathrm{ug} / \mathrm{L}$ \\
\hline & & & 1 & 110 & 1000 & u & & $\mathrm{ug} / \mathrm{L}$ \\
\hline & & & 1 & 100 & 1000 & u & & $\mathrm{ug} / \mathrm{L}$ \\
\hline & & & 1 & 91 & 500 & u & & $\mathrm{ug} / \mathrm{L}$ \\
\hline & & & 1 & 100 & 500 & u & & $\mathrm{ug} / \mathrm{L}$ \\
\hline & & & 1 & 40 & 1000 & u & & $\mathrm{ug} / \mathrm{L}$ \\
\hline & & & 1 & 52 & 500 & u & & $u g / L$ \\
\hline & & & 1 & 61 & 1000 & u & & ug/L \\
\hline & & & 1 & 120 & 1000 & u & & $\mathrm{ug} / \mathrm{L}$ \\
\hline & & & 1 & 57 & 500 & u & & ug/L \\
\hline & & & 1 & 45 & 500 & u & & ug/L \\
\hline & & & 1 & 110 & 500 & $u$ & & $u g / L$ \\
\hline & & & 1 & 150 & 500 & u & & $\mathrm{ug} / \mathrm{L}$ \\
\hline & & & 1 & 150 & 500 & u & & ug/L \\
\hline & & & 1 & 190 & 1000 & u & & $u g / L$ \\
\hline & & & 1 & 53 & 500 & $u$ & & $u g / L$ \\
\hline & & & 1 & 140 & 500 & u & & $u g / L$ \\
\hline & & & 1 & 70 & 500 & u & & $u g / L$ \\
\hline & & & 1 & 120 & 500 & $u$ & & $u g / L$ \\
\hline & & & 1 & 120 & 500 & u & & $\mathrm{ug} / \mathrm{L}$ \\
\hline & & & 1 & 61 & 500 & $u$ & & ug/L \\
\hline & & & 1 & 97 & 500 & u & & ug $/ \mathrm{L}$ \\
\hline & & & 1 & 69 & 500 & u & & $u g / L$ \\
\hline & & & 1 & 140 & 500 & u & & $\mathrm{ug} / \mathrm{L}$ \\
\hline & & & 1 & 160 & 500 & $u$ & & $u g / L$ \\
\hline
\end{tabular}

UNCLASSIFIED 
UNCLASSIFIED

LIMS 03.08 .048

04/12/2010 15:44

132649 Dibenzofuran

84662 Diethylphthalate

131113 Dimethylphthalate

206440 Fluoranthene

$86737 \quad$ Fluorene

118741 Hexachlorobenzene

77474 Hexachlorocyclopentadiene

67721 Hexachloroethane

193395 Indeno (1,2,3-cd)pyrene

78591 Isophorone

$621647 \quad \mathrm{~N}$-Nitroso-di-n-propylamine

$62759 \quad \mathrm{~N}$-Nitrosodimethylamine

$86306 \quad \mathrm{~N}$-Nitrosodiphenylamine

$91203 \quad$ Naphthalene

98953 Nitrobenzene

87865 Pentachlorophenol

85018 Phenanthrene

108952 Phenol

129000 Pyrene

\begin{tabular}{ll} 
TIC Id & TIC Name \\
\hdashline 000123422 & $\begin{array}{l}\text { 2-Pentanone, 4-hydroxy-4-methy)- (CAS) \$ } \\
\text { unknown } \\
\text { unknown } \\
\text { unknown } \\
\text { unknown }\end{array}$
\end{tabular}

Analytical Chemistry organization (Quality Services)

Page 2 of 2

P.o. Box 2009 oak Ridge, TN 378318244 (865) 574-2284 official Report

Cust Smpl Id: H2O 300 NEW

Lab Smp1 Id: A100690179

$\begin{array}{lllll}1 & 92 & 500 & \mathrm{u} & \mathrm{ug} / \mathrm{L}\end{array}$

$100-500$

$51-500$

$74 \quad 500$

$66 \quad 500$

$260 \quad 500$

$150-500$

$100 \quad 500$

$70 \quad 500$

$65 \quad 500$

$150 \quad 500$

$59-500$

$97-500$

48
54 $\quad 500$

$62 \quad 500$

$92 \quad 500$

Retention Estimated EPA

Time Unit Conc Qual Unit

$\mathrm{Fn}$

-1.--

$32.08 \quad \min$

33.74

6.72

8.71

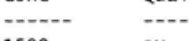

1500

740

5700

15000

400

ug/

ug/

$u g / L$

ug/L

$u g / L$

$\mathrm{ug} / \mathrm{L}$

$\mathrm{ug} / \mathrm{L}$

ug/

$\mathrm{ug} / \mathrm{L}$

ug/L

$\mathrm{ug} / \mathrm{L}$

$\mathrm{ug} / \mathrm{L}$

$u g / L$

$\mathrm{ug} / \mathrm{L}$

Sample Test comments: Target Compound(s) Initial calibration exceeding 15\% RSD:

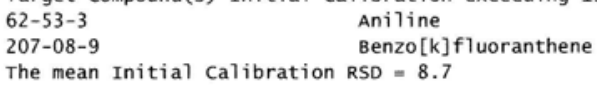

The mean Initial Calibration RSD $=8.7$

Comment objects:

EPA Qualifiers:

J - Estimated value.

N - Presumptive evidence of a compound. (GC/MS flag)

$U$ - Analyte analyzed for but undetected. Analyte result was below the contract required quantitation limit (CRQL).

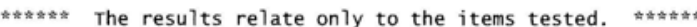

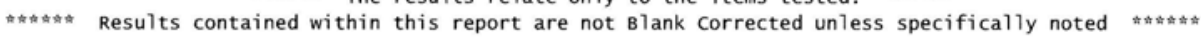

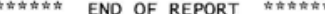

UNCLASSIFIED 
UNCLASSIFIED

LIMS 03.08.048

02/22/2010 07:03

Project: L MS MPO SCHUH SVOA

Customer: L P BURNETT B ddg 9995 MS-8189 (865) 576-9141

\begin{tabular}{|c|c|c|c|c|c|c|c|}
\hline Matrix & $\begin{array}{l}\text { Chain of } \\
\text { Custody }\end{array}$ & $\begin{array}{l}\text { Work Agreement } \\
\text { Number }\end{array}$ & $\begin{array}{l}\text { Charge } \\
\text { Number }\end{array}$ & $\begin{array}{l}\text { Date } \\
\text { Sampled }\end{array}$ & $\begin{array}{l}\text { Date } \\
\text { Received }\end{array}$ & $\begin{array}{l}\text { Date } \\
\text { Needed }\end{array}$ & $\begin{array}{l}\text { Date } \\
\text { Approved }\end{array}$ \\
\hline$\cdots-$ & ----- & --.-- & --- & - n-n- & ----- & -..-- & - \\
\hline LIQUID & 117929 & 81473 & $745 A 0221$ & $01 / 21 / 201000: 00$ & 01/21/2010 14:30 & $02 / 04 / 2010 \quad 23: 59$ & $02 / 22 / 2010 \quad 07: 03$ \\
\hline
\end{tabular}

Customer comments:

Lab comments:

Comment objects:
Proj Mgr: L P BURNETT (865) 576-9141
Sample status: APPROVED

Sample Approver: J T HOFFARTH/Data Approver
Analytical Chemistry organization (Quality Services)

Page 1 of 2

P.0, Box 2009 oak Ridge, TN 378318244 (865) 574-2284

Lab Smpl Id: A100250076

official Report

Cust Smp1 Id: HFIR water $250 \mathrm{Mrad}$

$\ll<<$ Organic $\gg \gg>>$

Rpt Basis: AR Test Req Cnt: 01

Analysis Method: SW846 8270C (Y50-AC-65-7316)

Approver: ] T HOFFARTH/Data Approver

QC Batch/File: QC10049010/5281/53758

$$
\begin{aligned}
& \text { Method/Date: SW846 } \\
& \text { Test Status: APPROVED } \\
& \text { HT Deadline: } 03 / 13 / 2010 \quad 23: 59 \\
& \text { Date Analyzed: } 02 / 15 / 2010 \quad 19: 42 \\
& \text { Lab Group: OYGCMS }
\end{aligned}
$$

\begin{tabular}{|c|c|c|c|c|c|c|c|c|}
\hline \multicolumn{3}{|c|}{ Customer } & \multirow{2}{*}{$\begin{array}{l}\text { Dilution } \\
\text { Factor }\end{array}$} & \multirow{2}{*}{$\begin{array}{l}\text { Lower } \\
\text { Limit }\end{array}$} & \multirow[b]{2}{*}{ Result } & \multirow{2}{*}{$\begin{array}{l}\text { EPA } \\
\text { Qual }\end{array}$} & \multirow[b]{2}{*}{ Confidence } & \multirow[b]{2}{*}{ Unit } \\
\hline HT & Lim & $\mathrm{Fn}$ & & & & & & \\
\hline-- & $\cdots$ & -- & $-\cdots$ & - & ----- & $m$ & - & (n) \\
\hline & & & 3 & 300 & 1500 & u & & $u g / L$ \\
\hline & & & 3 & 340 & 1500 & u & & ug $/ \mathrm{L}$ \\
\hline & & & 3 & 180 & 1500 & u & & ug/L \\
\hline & & & 3 & 510 & 1500 & u & & $\mathrm{ug} / \mathrm{L}$ \\
\hline & & & 3 & 530 & 1500 & $u$ & & ug/L \\
\hline & & & 3 & 580 & 1500 & $u$ & & $\mathrm{ug} / \mathrm{L}$ \\
\hline & & & 3 & 310 & 1500 & $u$ & & $\mathrm{ug} / \mathrm{L}$ \\
\hline & & & 3 & 290 & 1500 & u & & $\mathrm{ug} / \mathrm{L}$ \\
\hline & & & 3 & 300 & 1500 & u & & ug/L \\
\hline & & & 3 & 430 & 3000 & u & & $\mathrm{ug} / \mathrm{L}$ \\
\hline & & & 3 & 390 & 1500 & u & & $\mathrm{ug} / \mathrm{L}$ \\
\hline & & & 3 & 290 & 1500 & u & & $\mathrm{ug} / \mathrm{L}$ \\
\hline & & & 3 & 260 & 1500 & u & & ug/L \\
\hline & & & 3 & 300 & 1500 & $u$ & & ug/L \\
\hline & & & 3 & 320 & 3000 & u & & ug/L \\
\hline & & & 3 & 200 & 1500 & $u$ & & $u g / L$ \\
\hline & & & 3 & 290 & 1500 & u & & ug/L \\
\hline & & & 3 & 340 & 1500 & $u$ & & $\mathrm{ug} / \mathrm{L}$ \\
\hline & & & 3 & 240 & 1500 & u & & $u g / L$ \\
\hline & & & 3 & 150 & 3000 & $u$ & & ug/L \\
\hline & & & 3 & 350 & 3000 & $u$ & & $u g / L$ \\
\hline & & & 3 & 650 & 1500 & $u$ & & ug/L \\
\hline & & & 3 & 200 & 1500 & u & & $\mathrm{ug} / \mathrm{L}$ \\
\hline & & & 3 & 160 & 1500 & u & & $\mathrm{ug} / \mathrm{L}$ \\
\hline & & & 3 & 300 & 1500 & u & & $\mathrm{ug} / \mathrm{L}$ \\
\hline & & & 3 & 200 & 1500 & u & & ug/L \\
\hline & & & 3 & 330 & 3000 & $u$ & & $\mathrm{ug} / \mathrm{L}$ \\
\hline & & & 3 & 300 & 3000 & u & & $\mathrm{ug} / \mathrm{L}$ \\
\hline & & & 3 & 270 & 1500 & u & & ug/L \\
\hline & & & 3 & 310 & 1500 & u & & ug/L \\
\hline & & & 3 & 120 & 3000 & u & & ug/L \\
\hline & & & 3 & 160 & 1500 & $u$ & & ug/L \\
\hline & & & 3 & 180 & 3000 & $u$ & & ug/L \\
\hline & & & 3 & 370 & 3000 & $u$ & & $u g / L$ \\
\hline & & & 3 & 170 & 1500 & $u$ & & $\mathrm{ug} / \mathrm{L}$ \\
\hline & & & 3 & 140 & 1500 & u & & $\mathrm{ug} / \mathrm{L}$ \\
\hline & & & 3 & 330 & 1500 & $u$ & & $u g / L$ \\
\hline & & & 3 & 450 & 1500 & $u$ & & $\mathrm{ug} / \mathrm{L}$ \\
\hline & & & 3 & 440 & 1500 & u & & ug/L \\
\hline & & & 3 & 570 & 3000 & $u$ & & $u g / L$ \\
\hline & & & 3 & 160 & 1500 & $u$ & & ug/L \\
\hline & & & 3 & 410 & 1500 & $u$ & & ug/L \\
\hline & & & 3 & 210 & 1500 & u & & $u g / L$ \\
\hline & & & 3 & 370 & 1500 & u & & $\mathrm{ug} / \mathrm{L}$ \\
\hline & & & 3 & 370 & 1500 & $u$ & & $u g / L$ \\
\hline & & & 3 & 180 & 1500 & u & & ug/L \\
\hline & & & 3 & 290 & 1500 & u & & ug/L \\
\hline & & & 3 & 210 & 1500 & u & & ug/L \\
\hline & & & 3 & 420 & 1500 & u & & $\mathrm{ug} / \mathrm{L}$ \\
\hline & & & 3 & 480 & 1500 & u & & $g / L$ \\
\hline
\end{tabular}

Analyte Id Analyte Name

$\begin{array}{ll}---120821 & \text { 1,2,4-Trichlorobenzene } \\ 95501 & \text { 1,2-Dichlorobenzene } \\ 122667 & \text { 1,2-Diphenylhydrazine } \\ 541731 & \text { 1,3-Dichlorobenzene } \\ 106467 & \text { 1,4-Dichlorobenzene } \\ 95954 & \text { 2,4,5-Trichlorophenol } \\ 88062 & \text { 2,4,6-Trichlorophenol } \\ 120832 & \text { 2,4-Dichlorophenol } \\ 105679 & \text { 2,4-Dimethylphenol } \\ 51285 & \text { 2,4-Dinitrophenol } \\ 121142 & \text { 2,4-Dinitrotoluene } \\ 606202 & \text { 2,6-Dinitrotoluene } \\ 91587 & \text { 2-Chloronaphthalene } \\ 95578 & \text { 2-Chlorophenol } \\ 534521 & \text { 2-Methyl-4,6-dinitrophenol } \\ 91576 & \text { 2-Methylnaphthalene } \\ 95487 & \text { 2-Methylphenol } \\ 88744 & \text { 2-Nitrobenzenamine } \\ 88755 & \text { 2-Nitrophenol } \\ 91941 & \text { 3,3'-Dichlorobenzidine } \\ \text { N2799 } & \text { 3- and 4- Methylphenol } \\ 99092 & \text { 3-Nitrobenzenamine } \\ 101553 & \text { 4-Bromophenyl phenyl ether } \\ 59507 & \text { 4-Chloro-3-methylphenol } \\ 106478 & \text { 4-Chlorobenzenamine } \\ 7005723 & \text { 4-Chlorophenylphenyl ether } \\ 100016 & \text { 4-Nitrobenzenamine } \\ 100027 & \text { 4-Nitrophenol } \\ 83329 & \text { Acenaphthene } \\ 208968 & \text { Acenaphthylene } \\ 62533 & \text { Aniline } \\ 120127 & \text { Anthracene } \\ 100516 & \text { Benzenemethanol } \\ 92875 & \text { Benzidine } \\ 56553 & \text { Benzo(a)anthracene } \\ 50328 & \text { Benzo(a)pyrene } \\ 205992 & \text { Benzo(b)fluoranthene } \\ 191242 & \text { Benzo(ghi)perylene } \\ 207089 & \text { Benzo(k)fluoranthene } \\ 65850 & \text { Benzoic acid } \\ 111911 & \text { Bis(2-chloroethoxy)methane } \\ 111444 & \text { Bis(2-chloroethyl) ether } \\ 108601 & \text { Bis(2-chloroisopropyl) ether } \\ 117817 & \text { Bis(2-ethylhexyl)phthalate } \\ 85687 & \text { Butylbenzylphthalate } \\ 86748 & \text { Carbazole } \\ 218019 & \text { Chrysene } \\ 84742 & \text { Di-n-butylphthalate } \\ 117840 & \text { Di-n-octylphthalate } \\ 53703 & \text { Dibenz(a,h)anthracene } \\ & \end{array}$

UNCLASSIFIED 


\section{UNCLASSIFIED}

LIMS 03.08 .048

02/22/2010 07:03

$\begin{array}{ll}132649 & \text { Dibenzofuran } \\ 84662 & \text { Diethylphthalate } \\ 131113 & \text { Dimethylphthalate } \\ 206440 & \text { Fluoranthene } \\ 86737 & \text { Fluorene } \\ 118741 & \text { Hexachlorobenzene } \\ 77474 & \text { Hexachlorocyclopentadiene } \\ 67721 & \text { Hexachloroethane } \\ 193395 & \text { Indeno(1,2,3-cd)pyrene } \\ 78591 & \text { Isophorone } \\ 621647 & \text { N-Nitroso-di-n-propylamine } \\ 62759 & \text { N-Nitrosodimethylamine } \\ 86306 & \text { N-Nitrosodiphenylamine } \\ 91203 & \text { Naphthalene } \\ 98953 & \text { Nitrobenzene } \\ 87865 & \text { Pentachlorophenol } \\ 85018 & \text { Phenanthrene } \\ 108952 & \text { Phenol } \\ 129000 & \text { Pyrene } \\ & \\ \text { TIC Id } & \text { TIC Name } \\ ----- & ------ \\ & \text { unknown }\end{array}$

000123422 2-Pentanone, 4-hydroxy-4-methyl-(CAS) \$
Analytical Chemistry organization (Quality Services)

Page 2 of 2 P.0. Box 2009 oak Ridge, TN 378318244 (865) 574-2284 Lab Smpl Id: A100250076 official Report Cust Smp1 Id: HFIR water $250 \mathrm{Mrad}$

\begin{tabular}{|c|c|c|c|c|c|}
\hline 3 & 280 & 1500 & \multicolumn{2}{|l|}{$u$} & $\mathrm{ug} / \mathrm{L}$ \\
\hline 3 & 260 & 1500 & \multicolumn{2}{|l|}{$u$} & $\mathrm{ug} / \mathrm{L}$ \\
\hline 3 & 300 & 1500 & \multicolumn{3}{|r|}{$u g / L$} \\
\hline 3 & 150 & 1500 & \multicolumn{3}{|r|}{$\mathrm{ug} / \mathrm{L}$} \\
\hline 3 & 220 & 1500 & \multicolumn{3}{|r|}{$u g / L$} \\
\hline 3 & 200 & 1500 & \multicolumn{3}{|r|}{$\mathrm{ug} / \mathrm{L}$} \\
\hline 3 & 780 & 1500 & \multicolumn{3}{|r|}{$\mathrm{ug} / \mathrm{L}$} \\
\hline 3 & 450 & 1500 & \multicolumn{3}{|r|}{$\mathrm{ug} / \mathrm{L}$} \\
\hline 3 & 300 & 1500 & \multicolumn{3}{|r|}{$\mathrm{ug} / \mathrm{L}$} \\
\hline 3 & 210 & 1500 & \multicolumn{3}{|r|}{$u g / L$} \\
\hline 3 & 190 & 1500 & \multicolumn{3}{|r|}{$u g / L$} \\
\hline 3 & 450 & 1500 & \multicolumn{3}{|r|}{$u g / L$} \\
\hline 3 & 180 & 1500 & \multicolumn{3}{|r|}{$u g / L$} \\
\hline 3 & 290 & 1500 & \multicolumn{3}{|r|}{$\mathrm{ug} / \mathrm{L}$} \\
\hline 3 & 140 & 1500 & \multicolumn{3}{|r|}{$\mathrm{ug} / \mathrm{L}$} \\
\hline 3 & 160 & 3000 & \multicolumn{3}{|r|}{$\mathrm{ug} / \mathrm{L}$} \\
\hline 3 & 190 & 1500 & \multicolumn{3}{|r|}{ g/L } \\
\hline 3 & 280 & 1500 & \multicolumn{3}{|r|}{$u g / L$} \\
\hline 3 & 340 & 1500 & \multicolumn{3}{|r|}{$\mathrm{ug} / \mathrm{L}$} \\
\hline & \multicolumn{2}{|l|}{ Retention } & $\begin{array}{l}\text { Estimated } \\
\text { Conc }\end{array}$ & $\begin{array}{l}\text { EPA } \\
\text { Qual }\end{array}$ & Unit \\
\hline & -- & --- & --n-s & -... & -- \\
\hline & 6.73 & $\min$ & 4100 & J & $\mathrm{ug} / \mathrm{L}$ \\
\hline & 7.48 & $\min$ & 2500 & JN & $\mathrm{ug} / \mathrm{L}$ \\
\hline
\end{tabular}

Sample Test Comments: Target Compound(s) Initial calibration exceeding 15\% RSD:

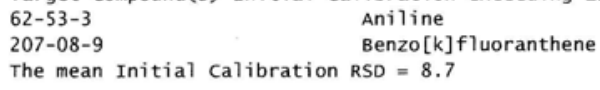

Comment objects:

EPA Qualifiers:

J - Estimated value.

$\mathrm{N}$ - Presumptive evidence of a compound. (GC/MS flag)

U - Analyte analyzed for but undetected. Analyte result was below the contract required quantitation limit (CRQL). 

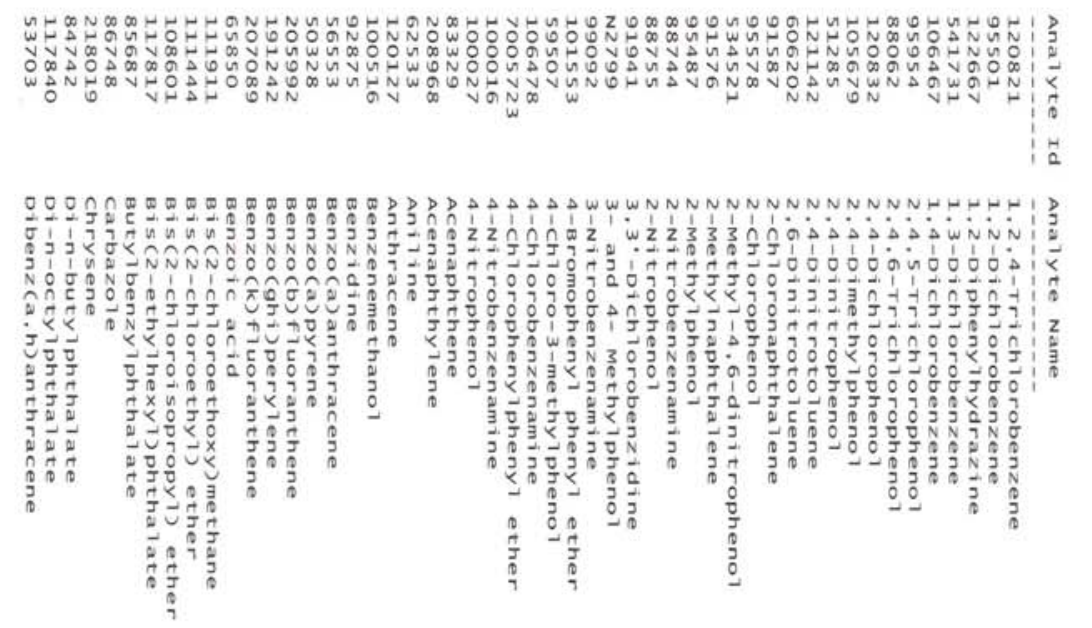

$c$
$Z$
$\cap$
$D$
$n$
$n$
$H$
$T$
$H$
$\Pi$
0

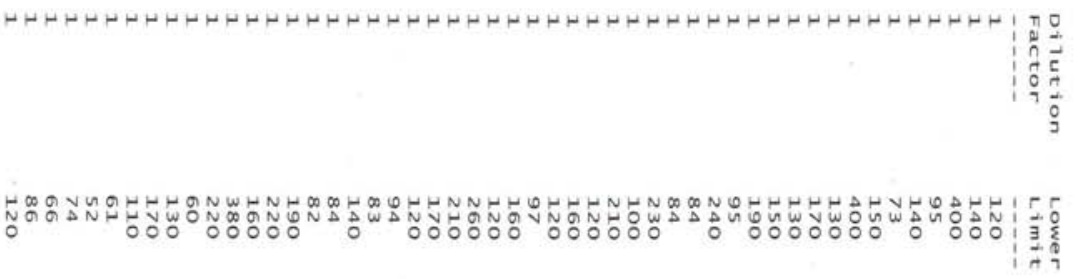

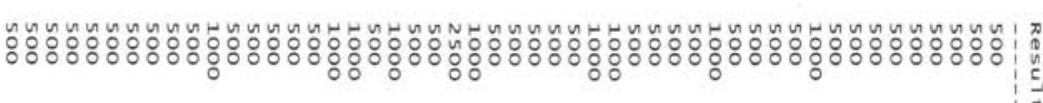

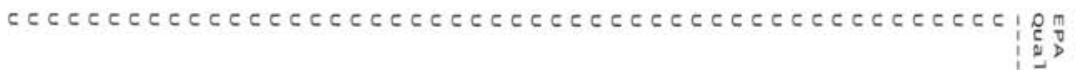

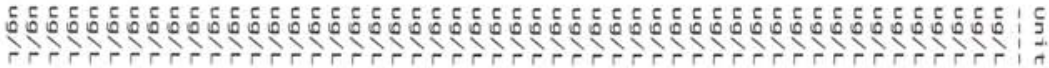

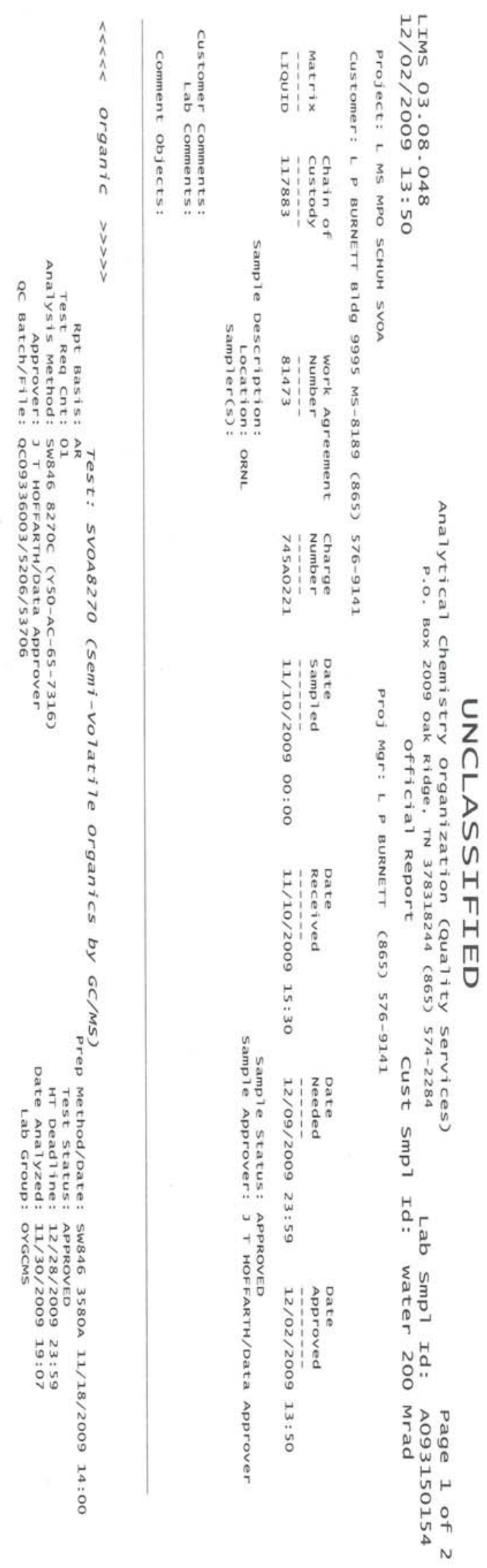


UNCLASSIFIED

LIMS 03.08 .048

$12 / 02 / 200913: 50$

132649 Dibenzofuran

84662 Diethylphthalate

131113 Dimethylphthalate

$206440 \quad$ Fluoranthene

$86737 \quad$ Fluorene

118741 Hexachlorobenzene

77474 Hexachlorocyclopentadiene

67721 Hexachloroethane

193395 Indeno $(1,2,3-c d)$ pyrene

78591 Isophorone

$621647 \quad \mathrm{~N}$-Nitroso-di-n-propylamine

$62759 \quad \mathrm{~N}$-Nitrosodimethylamine

$86306 \quad \mathrm{~N}$-Nitrosodiphenylamine

$91203 \quad$ Naphthalene

98953 Nitrobenzene

$87865 \quad$ Pentachloropheno

$85018 \quad$ Phenanthrene

108952 Pheno

129000 Pyrene

TIC Id TIC Name

unknown

000123422 2-Pentanone, 4-hydroxy-4-methy1- (CAS) \$
Analytical Chemistry organization (Quality Services)

Page 2 of 2 P.o. Box 2009 oak Ridge, TN 378318244 (865) 574-2284 official Report

Lab Smpl Id: A093150154

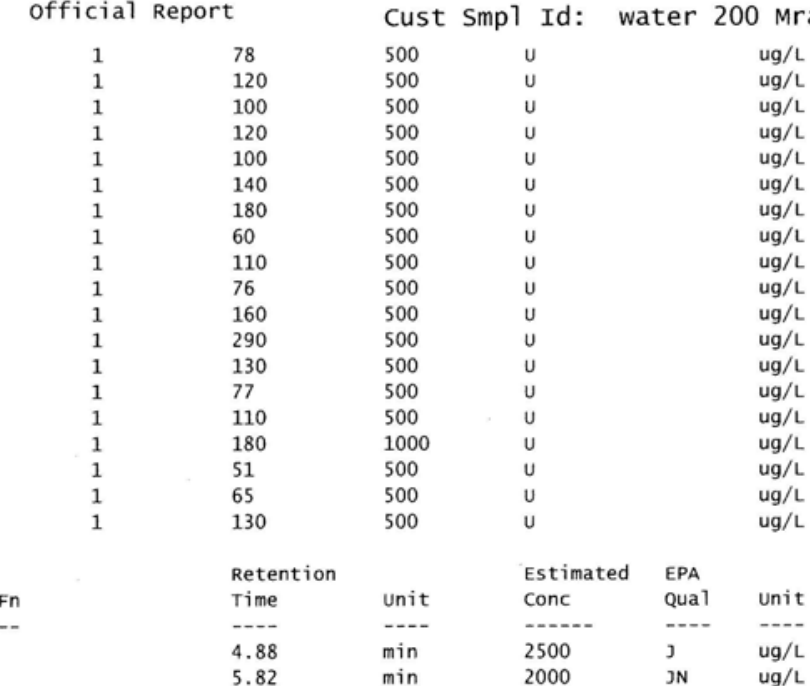

Sample Test comments: Target Compound(s) Initial calibration exceeding 15\% RSD:

$$
\begin{aligned}
& 111-44-4 \quad \text { bis }(2-\text { Chloroethy } 1) \text { ether } \\
& \text { The mean Initial calibration } R S D=9.3
\end{aligned}
$$

The mean Initial Calibration RSD $=9.3$

Comment objects:

EPA Qualifiers:

J - Estimated value.

N - Presumptive evidence of a compound. (GC/MS flag)

$U$ - Analyte analyzed for but undetected. Analyte result was below the contract required quantitation limit (CRQL).

\section{UNCLASSIFIED}


UNCLASSIFIED

Analytical Chemistry organization (Quality Services)

Page 1 of 2

LIMS 03.08 .048

P.o. Box 2009 oak Ridge, TN 378318244 (865) 574-2284

Lab Smpl Id: A100250074

02/22/2010 07:02

official Report

Cust Smpl Id: HFIR Water 150 Mrad

Project: L MS MPO SCHUH SVOA

Proj Mgr: L P BURNETT (865) 576-9141

Customer: L. P BURNETT Bldg 9995 MS-8189 (865) 576-9141

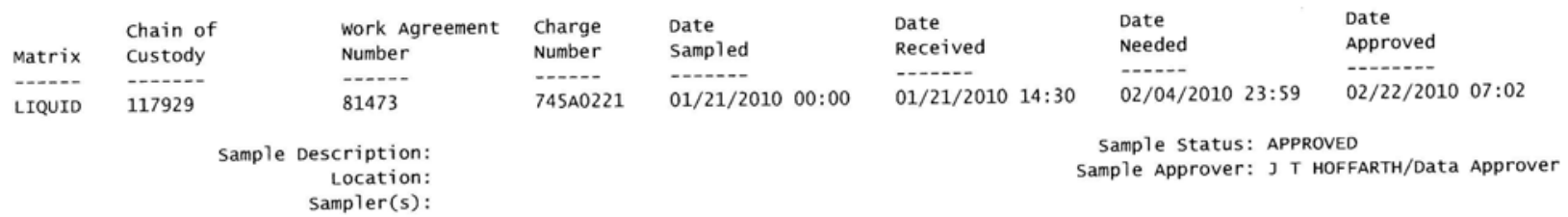

Customer comments:

Lab comments:

Comment objects:

$\ll<<$ organic $\gg \gg>$ Test: SVOA8270 (Semi-Volatile organics by GC/MS)

Rpt Basis: AR Test Req Cnt: 01

Analysis Method: SW846 8270C (Y50-AC-65-7316)

Approver: ] T HOFFARTH/Data Approver

QC Batch/File: QC10049010/5281/53758
Prep Method/Date: SW846 3580A 02/01/2010 13:00

Test Status: APPROVED

HT Deadline: 03/13/2010 23:59

Date Analyzed: 02/15/2010 18:10

Lab Group: OYGCMS

\begin{tabular}{|c|c|}
\hline Analyte Id & Analyte Name \\
\hline --n-n-- & - \\
\hline 120821 & 1,2,4-Trich1orobenzene \\
\hline 95501 & 1,2-Dichlorobenzene \\
\hline 122667 & 1,2-Diphenylhydrazine \\
\hline 541731 & 1,3-Dichlorobenzene \\
\hline 106467 & 1,4-Dichlorobenzene \\
\hline 95954 & 2,4,5-Trichlorophenol \\
\hline 88062 & 2,4,6-Trichlorophenol \\
\hline 120832 & 2,4-Dichlorophenol \\
\hline 105679 & 2,4-Dimethylphenol \\
\hline 51285 & 2,4-Dinitrophenol \\
\hline 121142 & 2,4-Dinitrotoluene \\
\hline 606202 & 2,6-Dinitrotoluene \\
\hline 91587 & 2-Chloronaphthalene \\
\hline 95578 & 2-Chlorophenol \\
\hline 534521 & 2-Methy $1-4,6$-dinitropheno 1 \\
\hline 91576 & 2-Methylnaphthalene \\
\hline 95487 & 2-Methylphenol \\
\hline 88744 & 2-Nitrobenzenamine \\
\hline 88755 & 2-Nitrophenol \\
\hline 91941 & $3,3^{\prime}$-Dichlorobenzidine \\
\hline N2799 & 3- and 4- Methylphenol \\
\hline 99092 & 3-Nitrobenzenamine \\
\hline 101553 & 4-Bromopheny 1 pheny 1 ether \\
\hline 59507 & 4-Chloro-3-methylphenol \\
\hline 106478 & 4-Chlorobenzenamine \\
\hline 7005723 & 4-Ch 1 oropheny 1 pheny 1 ether \\
\hline 100016 & 4-Nitrobenzenamine \\
\hline 100027 & 4-Nitrophenol \\
\hline 83329 & Acenaphthene \\
\hline 208968 & Acenaphthylene \\
\hline 62533 & Aniline \\
\hline 120127 & Anthracene \\
\hline 100516 & Benzenemethanol \\
\hline 92875 & Benzidine \\
\hline 56553 & Benzo (a) anthracene \\
\hline 50328 & Benzo(a)pyrene \\
\hline 205992 & Benzo(b) fluoranthene \\
\hline 191242 & Benzo(ghi)perylene \\
\hline 207089 & Benzo $(k)$ fluoranthene \\
\hline 65850 & Benzoic acid \\
\hline 111911 & Bis (2-chloroethoxy)methane \\
\hline 111444 & Bis (2-chloroethyl) ether \\
\hline 108601 & Bis (2-chloroisopropy 1 ) ether \\
\hline 117817 & Bis (2-ethylhexy1)phthalate \\
\hline 85687 & Butylbenzylphthalate \\
\hline 86748 & Carbazole \\
\hline 218019 & Chrysene \\
\hline 84742 & Di-n-butylphthalate \\
\hline 117840 & Di-n-octylphthalate \\
\hline 53703 & Dibenz $(a, h)$ anthracene \\
\hline
\end{tabular}

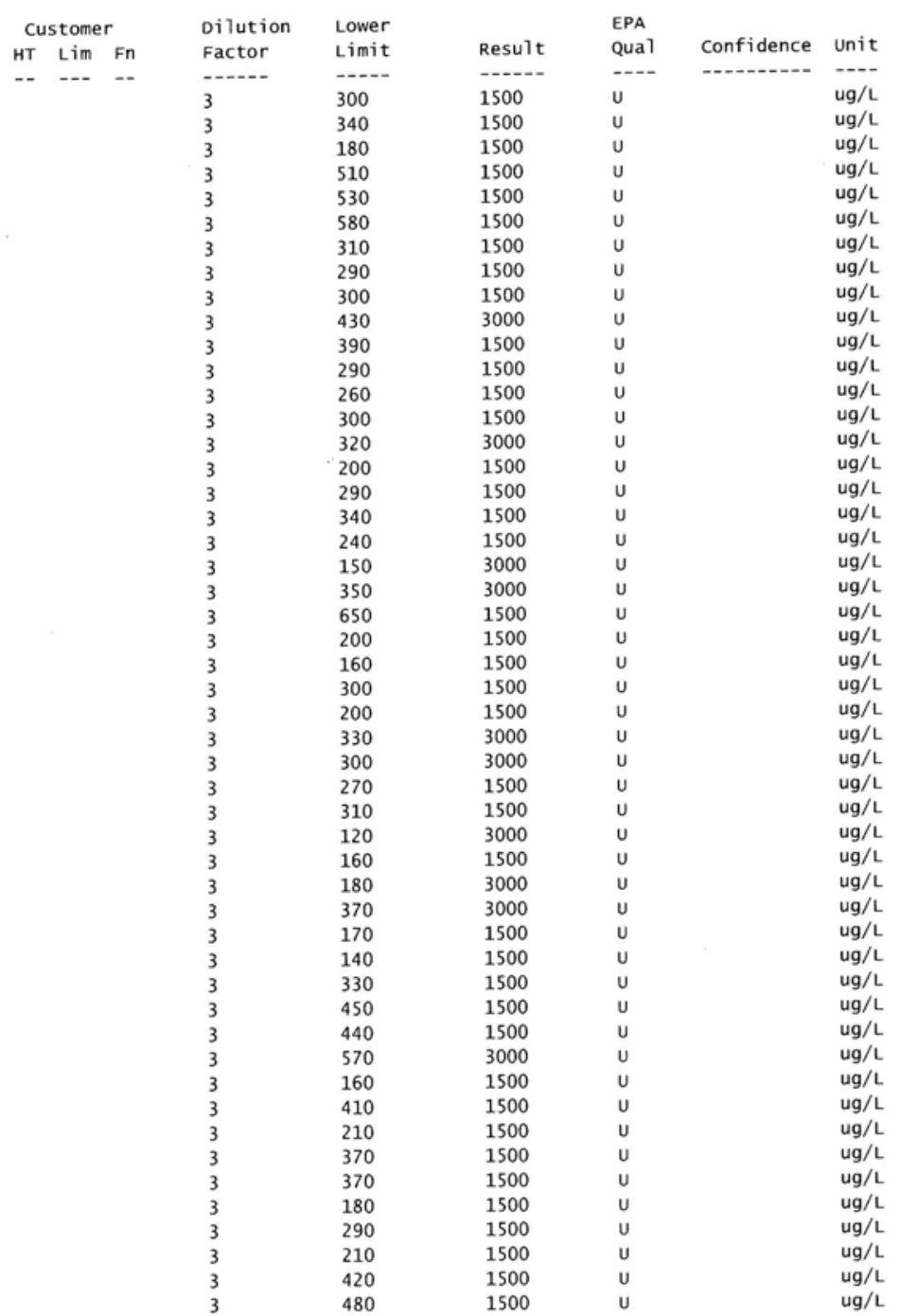

UNCLASSIFIED 


\section{UNCLASSIFIED}

LIMS 03.08 .048

02/22/2010 07:02

$\begin{array}{ll}132649 & \text { Dibenzofuran } \\ 84662 & \text { Diethylphthalate } \\ 131113 & \text { Dimethylphthalate } \\ 206440 & \text { Fluoranthene } \\ 86737 & \text { Fluorene } \\ 118741 & \text { Hexachlorobenzene } \\ 77474 & \text { Hexachlorocyclopentadiene } \\ 67721 & \text { Hexachloroethane } \\ 193395 & \text { Indeno(1,2,3-cd)pyrene } \\ 78591 & \text { Isophorone } \\ 621647 & \text { N-Nitroso-di-n-propylamine } \\ 62759 & \text { N-Nitrosodimethylamine } \\ 86306 & \text { N-Nitrosodiphenylamine } \\ 91203 & \text { Naphthalene } \\ 98953 & \text { Nitrobenzene } \\ 87865 & \text { Pentachlorophenol } \\ 85018 & \text { Phenanthrene } \\ 108952 & \text { Phenol } \\ 129000 & \text { Pyrene } \\ & \\ & \\ \text { TIC Id } & \text { TIC Name } \\ ---- & ------ \\ & \text { unknown }\end{array}$

000123422 2-Pentanone, 4-hydroxy-4-methy 1-
Analytical Chemistry Organization (Quality Services) P.o. Box 2009 oak Ridge, TN 378318244 (865) 574-2284 official Report

\begin{tabular}{|c|c|c|c|}
\hline & 3 & 280 & 1500 \\
\hline & 3 & 260 & 1500 \\
\hline & 3 & 300 & 1500 \\
\hline & 3 & 150 & 1500 \\
\hline & 3 & 220 & 1500 \\
\hline & 3 & 200 & 1500 \\
\hline & 3 & 780 & 1500 \\
\hline & 3 & 450 & 1500 \\
\hline & 3 & 300 & 1500 \\
\hline & 3 & 210 & 1500 \\
\hline & 3 & 190 & 1500 \\
\hline & 3 & 450 & 1500 \\
\hline & 3 & 180 & 1500 \\
\hline & 3 & 290 & 1500 \\
\hline & 3 & 140 & 1500 \\
\hline & 3 & 160 & 3000 \\
\hline & 3 & 190 & 1500 \\
\hline & 3 & 280 & 1500 \\
\hline & 3 & 340 & 1500 \\
\hline \multirow{2}{*}{\multicolumn{2}{|c|}{$\mathrm{Fn}$}} & Retention & \\
\hline & & Time & Unit \\
\hline \multirow[t]{3}{*}{--} & & ---- & $\cdots$ \\
\hline & & 6.74 & $\min$ \\
\hline & & 7.48 & $\min$ \\
\hline
\end{tabular}

Page 2 of 2

Lab Smpl Id: A100250074

Smp1 Id: HFIR water $150 \mathrm{Mrad}$

\begin{tabular}{|c|c|c|}
\hline u & & ug/L \\
\hline$u$ & & $\mathrm{ug} / \mathrm{L}$ \\
\hline u & & $\mathrm{ug} / \mathrm{L}$ \\
\hline$u$ & & ug/L \\
\hline u & & $\mathrm{ug} / \mathrm{L}$ \\
\hline u & & $\mathrm{ug} / \mathrm{L}$ \\
\hline u & & $u g / L$ \\
\hline u & & $\mathrm{ug} / \mathrm{L}$ \\
\hline$u$ & & $\mathrm{ug} / \mathrm{L}$ \\
\hline$u$ & & $\mathrm{ug} / \mathrm{L}$ \\
\hline$u$ & & $u g / L$ \\
\hline$u$ & & $u g / L$ \\
\hline$u$ & & $u g / L$ \\
\hline$u$ & & $\mathrm{ug} / \mathrm{L}$ \\
\hline$u$ & & $u g / L$ \\
\hline u & & $\mathrm{ug} / \mathrm{L}$ \\
\hline u & & $u g / L$ \\
\hline$u$ & & $\mathrm{ug} / \mathrm{L}$ \\
\hline$u$ & & $u g / L$ \\
\hline $\begin{array}{l}\text { Estimated } \\
\text { Conc }\end{array}$ & $\begin{array}{l}\text { EPA } \\
\text { Qua } 7\end{array}$ & Unit \\
\hline -....- & -- & $\cdots$ \\
\hline 2500 & J & $u g / L$ \\
\hline 2100 & JN & $u g / L$ \\
\hline
\end{tabular}

Sample Test Comments: Target Compound(s) Initial Calibration exceeding 15\% RSD:

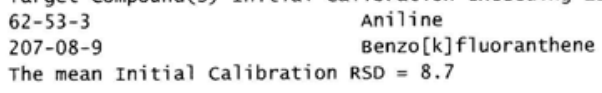

Comment objects:

EPA QUa7ifiers:

J - Estimated value.

N - Presumptive evidence of a compound. (GC/MS flag)

$U$ - Analyte analyzed for but undetected. Analyte result was below the contract required quantitation limit (CRQL).

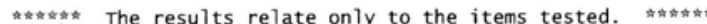

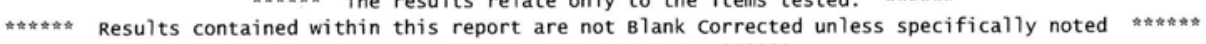

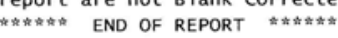

UNCLASSIFIED 


\section{UNCLASSIFIED}

LIMS 03.08 .048

05/06/2010 06:22
Analytical Chemistry organization (Quality Services)

P.o. Box 2009 oak Ridge, TN 378318244 (865) 574-2284

official Report
Page 1 of 2

Lab Smpl Id: A100910405

Proj Mgr: L P BURNETT (865) 576-9141 Cust Smpl Id: HFIR 01dwater 100Mrad

Customer: L P BURNETT Bldg 9995 MS-8189 (865) 576-9141

\begin{tabular}{|c|c|c|c|c|c|c|c|}
\hline Matrix & $\begin{array}{l}\text { Chain of } \\
\text { Custody }\end{array}$ & $\begin{array}{l}\text { Work Agreement } \\
\text { Number }\end{array}$ & $\begin{array}{l}\text { Charge } \\
\text { Number }\end{array}$ & $\begin{array}{l}\text { Date } \\
\text { Sampled }\end{array}$ & $\begin{array}{l}\text { Date } \\
\text { Received }\end{array}$ & $\begin{array}{l}\text { Date } \\
\text { Needed }\end{array}$ & $\begin{array}{l}\text { Date } \\
\text { Approved }\end{array}$ \\
\hline --n- & -...-- & ----- & $-\ldots$ & - & 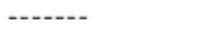 & --.-- & - - \\
\hline LIQUID & 117946 & 81473 & $745 A 0221$ & 03/31/2010 08:00 & $04 / 01 / 2010 \quad 14: 15$ & 04/15/2010 23:59 & $05 / 06 / 2010 \quad 06: 22$ \\
\hline
\end{tabular}

Customer comments:

Lab Comments:

Comment objects:

Rpt Basis: AR Test Req Cnt: 01

Analysis Method: SW846 8270 C (Y50-AC-65-7316)

Approver: J T HOFFARTH/Data Approver

QC Batch/File: QC10124002/5363/53833
Prep Method/Date: Sw846 3580A 04/06/2010 10:00 Test Status: APPROVED

HT Deadline: 05/16/2010 23:59

Date Analyzed: 04/27/2010 23:10 Lab Group: OYGCMS

\begin{tabular}{|c|c|}
\hline Analyte Id & Analyte Name \\
\hline 120821 & 1,2,4-Trichlorobenzene \\
\hline 95501 & 1,2-Dichlorobenzene \\
\hline 122667 & 1,2-Diphenylhydrazine \\
\hline 541731 & 1,3-Dichlorobenzene \\
\hline 106467 & 1,4-Dichlorobenzene \\
\hline 95954 & $2,4,5$-Trichloropheno 1 \\
\hline 88062 & 2,4,6-Trichloropheno 1 \\
\hline 120832 & 2,4-Dichlorophenol \\
\hline 105679 & 2,4-Dimethylphenol \\
\hline 51285 & 2,4-Dinitrophenol \\
\hline 121142 & 2,4-Dinitrotoluene \\
\hline 606202 & 2,6-Dinitrotoluene \\
\hline 91587 & 2-Chloronaphthalene \\
\hline 95578 & 2-Chlorophenol \\
\hline 534521 & 2-Methy $7-4,6$-dinitrophenol \\
\hline 91576 & 2-Methylnaphthalene \\
\hline 95487 & 2-Methylphenol \\
\hline 88744 & 2-Nitrobenzenamine \\
\hline 88755 & 2-Nitrophenol \\
\hline 91941 & 3, 3'-Dichlorobenzidine \\
\hline N2799 & 3- and 4- Methylphenol \\
\hline 99092 & 3-Nitrobenzenamine \\
\hline 101553 & 4-Bromopheny 1 pheny 1 ether \\
\hline 59507 & 4-chloro-3-methylphenol \\
\hline 106478 & 4-Chlorobenzenamine \\
\hline 7005723 & 4-Ch1oropheny 1 pheny 1 ether \\
\hline 100016 & 4-Nitrobenzenamine \\
\hline 100027 & 4-Nitrophenol \\
\hline 83329 & Acenaphthene \\
\hline 208968 & Acenaphthylene \\
\hline 62533 & Aniline \\
\hline 120127 & Anthracene \\
\hline 100516 & Benzenemethanol \\
\hline 92875 & Benzidine \\
\hline 56553 & Benzo(a) anthracene \\
\hline 50328 & Benzo(a)pyrene \\
\hline 205992 & Benzo (b) fluoranthene \\
\hline 191242 & Benzo(ghi)perylene \\
\hline 207089 & Benzo $(k)$ fluoranthene \\
\hline 65850 & Benzoic acid \\
\hline 111911 & Bis(2-chloroethoxy)methane \\
\hline 111444 & Bis (2-chloroethy 1$)$ ether \\
\hline 108601 & Bis (2-chloroisopropyl) ether \\
\hline 117817 & Bis (2-ethylhexy1)phthalate \\
\hline 85687 & Butylbenzy]phthalate \\
\hline 86748 & Carbazole \\
\hline 218019 & Chrysene \\
\hline 84742 & Di-n-buty 1 phthalate \\
\hline 117840 & Di-n-octylphthalate \\
\hline 53703 & Dibenz $(a, h)$ anthracene \\
\hline
\end{tabular}

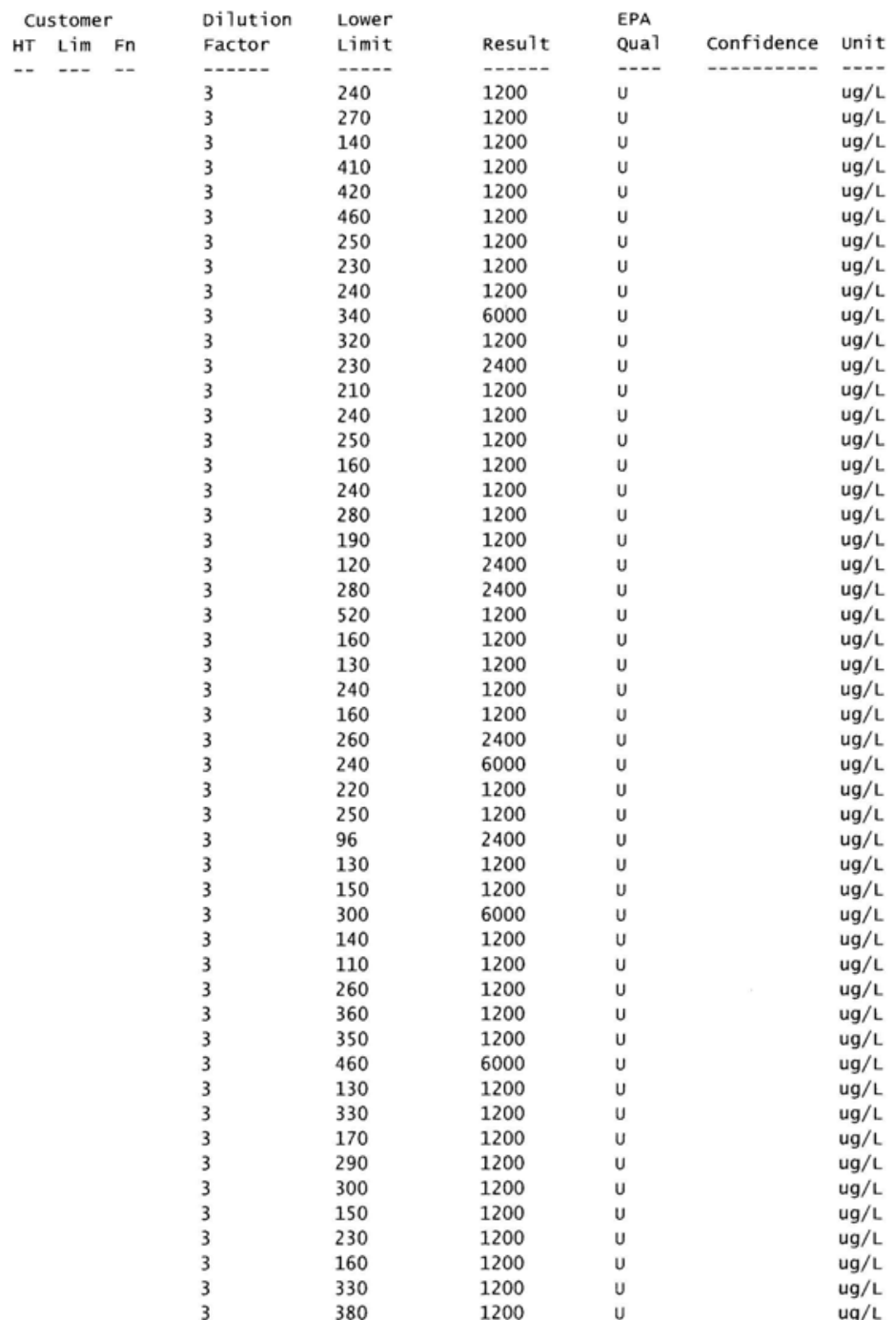

UNCLASSIFIED 


\section{UNCLASSIFIED}

LIMS 03.08 .048

05/06/2010 06:22

132649 Dibenzofuran

84662 Diethylphthalate

131113 Dimethylphthalate

$206440 \quad$ Fluoranthene

86737 Fluorene

118741 Hexachlorobenzene

77474 Hexachlorocyclopentadiene

67721 Hexachloroethane

193395 Indeno(1,2,3-cd)pyrene

78591 Isophorone

621647 N-Nitroso-di-n-propylamine

$62759 \quad \mathrm{~N}$-Nitrosodimethylamine

$86306 \quad \mathrm{~N}$-Nitrosodiphenylamine

$91203 \quad$ Naphthalene

98953 Nitrobenzene

87865 Pentachlorophenol

$85018 \quad$ Phenanthrene

108952 Phenol

129000 Pyrene

TIC Id TIC Name

$.000000000 \quad$ 2-NITROPHENOL-D4

unknown

unknown
Analytical Chemistry Organization (Quality Services)

P.0. Box 2009 oak Ridge, TN 378318244 (865) 574-2284

Page 2 of 2 official Report

Lab Smpl Id:

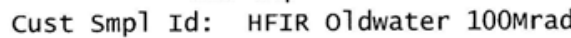

\begin{tabular}{|c|c|c|c|c|}
\hline 220 & 1200 & u & & ug/L \\
\hline 210 & 1200 & u & & ug/L \\
\hline 240 & 1200 & u & & $\mathrm{ug} / \mathrm{L}$ \\
\hline 120 & 1200 & u & & $\mathrm{ug} / \mathrm{L}$ \\
\hline 180 & 1200 & u & & $u g / L$ \\
\hline 160 & 1200 & u & & $u g / L$ \\
\hline 620 & 1200 & u & & $\mathrm{ug} / \mathrm{L}$ \\
\hline 360 & 1200 & u & & $u g / L$ \\
\hline 240 & 1200 & $u$ & & $u g / L$ \\
\hline 170 & 1200 & u & & $u g / L$ \\
\hline 160 & 1200 & u & & $u g / L$ \\
\hline 360 & 1200 & u & & $u g / L$ \\
\hline 140 & 1200 & u & & $\mathrm{ug} / \mathrm{L}$ \\
\hline 230 & 1200 & u & & $u g / L$ \\
\hline 120 & 1200 & u & & $u g / L$ \\
\hline 130 & 2400 & u & & $\mathrm{ug} / \mathrm{L}$ \\
\hline 150 & 1200 & $u$ & & $u g / L$ \\
\hline 220 & 1200 & u & & ug/L \\
\hline 270 & 1200 & $u$ & & $u g / L$ \\
\hline $\begin{array}{l}\text { Retention } \\
\text { Time }\end{array}$ & Unit & $\begin{array}{l}\text { Estimated } \\
\text { Conc }\end{array}$ & $\begin{array}{l}\text { EPA } \\
\text { Qua 1 }\end{array}$ & Unit \\
\hline-- & $--\cdot$ & -..-- & --- & $\cdots$ \\
\hline 13.06 & $\min$ & 1300 & $\mathrm{JN}$ & $u g / L$ \\
\hline .5 & $\min$ & 580 & $\mathrm{~J}$ & $u g / L$ \\
\hline & $\min$ & 510 & 了 & \\
\hline
\end{tabular}

Sample Test comments: Target Compound(s) Initial Calibration exceeding 15\% RSD:

$\begin{array}{ll}65-85-0 & \text { Benzoic acid } \\ 90-13-1 & \text { 1-Chloronaphthalene } \\ 51-28-5 & 2,4-D i n i t r o p h e n o 1 \\ 100-02-7 & 4-N i t r o p h e n o l \\ 92-87-5 & \text { Benzidine } \\ \text { The mean Initial Calibration RSD }=10.6\end{array}$

Comment objects:

EPA Qualifiers:

J - Estimated value.

$\mathrm{N}$ - Presumptive evidence of a compound. (GC/MS flag)

$U$ - Analyte analyzed for but undetected. Analyte result was below the contract required quantitation limit (CRQL).

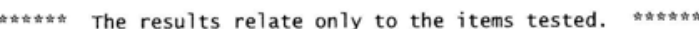

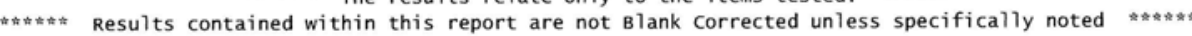

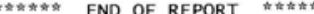

UNCLASSIFIED 


\section{UNCLASSIFIED}

LIMS 03.08.048

05/06/2010 06:21
Analytical Chemistry Organization (Quality Services)

P.O. Box 2009 oak Ridge, TN 378318244 (865) 574-2284

Page 1 of 2
Cust Smpl Id: HFIR oldwater 50Mrad

Project: L MS MPO SCHUH SVOA

$$
\text { official Report }
$$

Proj Mgr: L P BURNETT (865) 576-9141

Customer: L P BURNETT B 1dg 9995 MS-8189 (865) 576-9141

\begin{tabular}{|c|c|c|c|c|c|c|c|}
\hline Matrix & $\begin{array}{l}\text { Chain of } \\
\text { Custody }\end{array}$ & $\begin{array}{l}\text { Work Agreement } \\
\text { Number }\end{array}$ & $\begin{array}{l}\text { Charge } \\
\text { Number }\end{array}$ & $\begin{array}{l}\text { Date } \\
\text { Sampled }\end{array}$ & $\begin{array}{l}\text { Date } \\
\text { Received }\end{array}$ & $\begin{array}{l}\text { Date } \\
\text { Needed }\end{array}$ & $\begin{array}{l}\text { Date } \\
\text { Approved }\end{array}$ \\
\hline 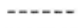 & $-\cdots-$ & $-\cdots-$ & $\cdots-$ & $-\cdots-$ & - n- & $\cdots-$ & 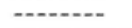 \\
\hline LIQUID & 117946 & 81473 & $745 A 0221$ & 03/31/2010 08:00 & $04 / 01 / 2010 \quad 14: 15$ & $04 / 15 / 2010 \quad 23: 59$ & $05 / 06 / 2010$ \\
\hline
\end{tabular}

Customer Comments:

Lab Comments:

Comment objects:

$\ll<$ Organic $\gg \gg>$ Test: SVOA8270 (Semi-volatile organics by GC/MS)

Rpt Basis: AR Test Req Cnt: 01

Analysis Method: SW846 8270C (Y50-AC-65-7316)

Approver: J T HOFFARTH/Data Approver

QC Batch/File: QC10124002/5363/53833
Prep Method/Date: SW846 3580A 04/06/2010 10:00 Test Status: APPROVED

HT Deadline: $05 / 16 / 201023: 59$

Date Analyzed: 04/27/2010 22:22

Lab Group: OYGCMS

$\begin{array}{ll}\text { Analyte Id } & \text { Analyte Name } \\ ---------- \\ 120821 & \text { 1,2,4-Trichlorobenzene } \\ 95501 & \text { 1,2-Dichlorobenzene } \\ 122667 & \text { 1,2-Diphenylhydrazine } \\ 541731 & \text { 1,3-Dichlorobenzene } \\ 106467 & \text { 1,4-Dichlorobenzene } \\ 95954 & \text { 2,4,5-Trichlorophenol } \\ 88062 & \text { 2,4,6-Trichlorophenol } \\ 120832 & \text { 2,4-Dichlorophenol } \\ 105679 & \text { 2,4-Dimethylphenol } \\ 51285 & \text { 2,4-Dinitrophenol } \\ 121142 & \text { 2,4-Dinitrotoluene } \\ 606202 & \text { 2,6-Dinitrotoluene } \\ 91587 & \text { 2-Chloronaphthalene } \\ 95578 & \text { 2-Chlorophenol } \\ 534521 & \text { 2-Methyl-4,6-dinitrophenol } \\ 91576 & \text { 2-Methylnaphthalene } \\ 95487 & \text { 2-Methylphenol } \\ 88744 & \text { 2-Nitrobenzenamine } \\ 88755 & \text { 2-Nitrophenol } \\ 91941 & \text { 3,3'-Dichlorobenzidine } \\ \text { N2799 } & \text { 3- and 4- Methylphenol } \\ 99092 & \text { 3-Nitrobenzenamine } \\ 101553 & \text { 4-Bromophenyl phenyl ether } \\ 59507 & \text { 4-Chloro-3-methylphenol } \\ 106478 & \text { 4-Chlorobenzenamine } \\ 7005723 & \text { 4-Chlorophenylphenyl ether } \\ 100016 & \text { 4-Nitrobenzenamine } \\ 100027 & \text { 4-Nitrophenol } \\ 83329 & \text { Acenaphthene } \\ 208968 & \text { Acenaphthylene } \\ 62533 & \text { Aniline } \\ 120127 & \text { Anthracene } \\ 100516 & \text { Benzenemethanol } \\ 92875 & \text { Benzidine } \\ 56553 & \text { Benzo(a)anthracene } \\ 50328 & \text { Benzo(a)pyrene } \\ 205992 & \text { Benzo(b)fluoranthene } \\ 191242 & \text { Benzo(ghi)perylene } \\ 207089 & \text { Benzo(k)fluoranthene } \\ 65850 & \text { Benzoic acid } \\ 111911 & \text { Bis(2-chloroethoxy)methane } \\ 111444 & \text { Bis(2-chloroethy1) ether } \\ 108601 & \text { Bis(2-chloroisopropyl) ether } \\ 117817 & \text { Bis(2-ethylhexyl)phthalate } \\ 85687 & \text { Butylbenzylphthalate } \\ 86748 & \text { Carbazole } \\ 218019 & \text { Chrysene } \\ 84742 & \text { Di-n-butylphthalate } \\ 117840 & \text { Di-n-octylphthalate } \\ 53703 & \text { Dibenz(a,h)anthracene } \\ & \\ & \end{array}$

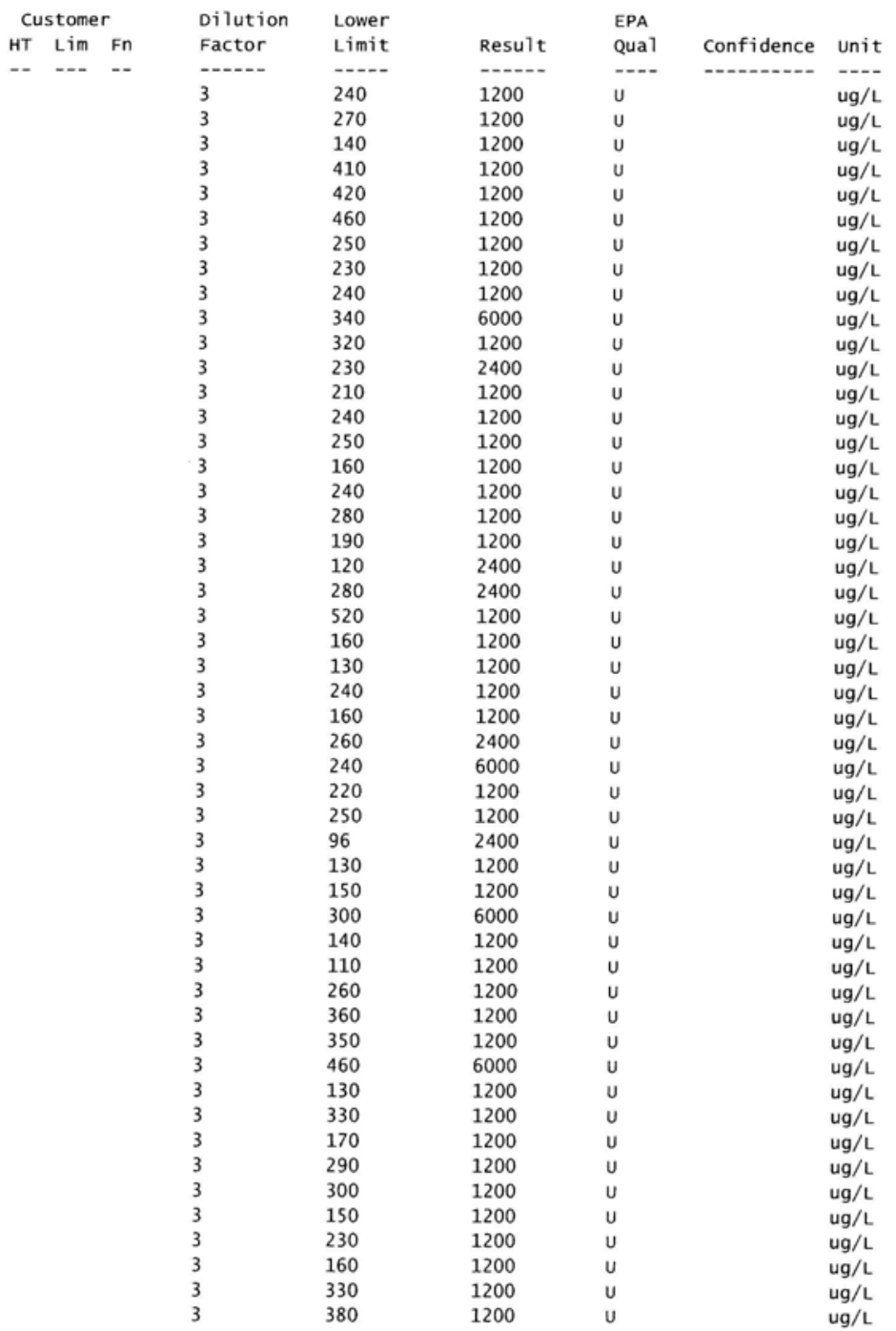

UNCLASSIFIED 


\section{UNCLASSIFIED}

LIMS 03.08 .048

05/06/2010 06:21

\begin{tabular}{|c|c|}
\hline 132649 & Dibenzofuran \\
\hline 84662 & Diethylphthalate \\
\hline 131113 & Dimethylphthalate \\
\hline 206440 & Fluoranthene \\
\hline 86737 & Fluorene \\
\hline 118741 & Hexachlorobenzene \\
\hline 77474 & Hexachlorocyclopentadiene \\
\hline 67721 & Hexachloroethane \\
\hline 193395 & Indeno $(1,2,3-c d)$ pyrene \\
\hline 78591 & Isophorone \\
\hline 621647 & $\mathrm{~N}$-Nitroso-di-n-propylamine \\
\hline 62759 & $\mathrm{~N}$-Nitrosodimethylamine \\
\hline 86306 & $\mathrm{~N}$-Nitrosodiphenylamine \\
\hline 91203 & Naphthalene \\
\hline 98953 & Ni trobenzene \\
\hline 87865 & Pentach1oropheno 1 \\
\hline 85018 & Phenanthrene \\
\hline 108952 & Phenol \\
\hline 129000 & Pyrene \\
\hline TIC Id & TIC Name \\
\hline & \\
\hline 000000000 & 2-NITROPHENOL-D4 \\
\hline
\end{tabular}

Analytical Chemistry organization (Quality Services) P.o. Box 2009 oak Ridge, TN 378318244 (865) 574-2284 official Report

$\begin{array}{ll}220 & 1200 \\ 210 & 1200 \\ 240 & 1200 \\ 120 & 1200 \\ 180 & 1200 \\ 160 & 1200 \\ 620 & 1200 \\ 360 & 1200 \\ 240 & 1200 \\ 170 & 1200 \\ 160 & 1200 \\ 360 & 1200 \\ 140 & 1200 \\ 230 & 1200 \\ 120 & 1200 \\ 130 & 2400 \\ 150 & 1200 \\ 220 & 1200 \\ 270 & 1200 \\ & \\ \text { Retention } & \\ \text { Time } & \text { unit } \\ --- \text {. } & --- \\ 13.05 & \text { min }\end{array}$

Page 2 of 2

Lab Smpl Id: A100910404 Cust Smpl Id: HFIR Oldwater 50Mrad

$1200 \quad \mathrm{ug} / \mathrm{L}$ $1200 \quad \mathrm{~V}-200$ $1200 \quad u \quad u g / L$ $1200 \quad$ u ug/L $1200 \quad$ u ug/L $1200 \quad$ U ug/L U $1200 \quad$ U ug/L $1200 \quad$ u $u g / L$ $1200 \quad$ u $\quad$ ug/L 1200 u ug/L 1200 u ug/L $2400 \quad u \quad u g / L$ $1200 \quad-\quad U$ $u \quad u g / L$ Estimated EPA Fn Unit Conc Qual unit

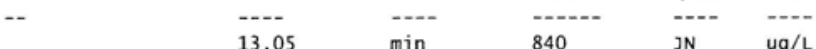

Sample Test Comments: Target Compound(s) Initial Calibration exceeding 15\% RSD:

$\begin{array}{ll}65-85-0 & \text { Benzoic acid } \\ 90-13-1 & \text { 1-Chloronaphthalene } \\ 51-28-5 & 2,4-\text { Dinitrophenol } \\ 100-02-7 & 4-\text { Nitrophenol } \\ 92-87-5 & \text { Benzidine } \\ \text { The mean Initial Calibration RSD }=10.6\end{array}$

Comment objects:

EPA Qualifiers:

J - Estimated value.

$\mathrm{N}$ - Presumptive evidence of a compound. (GC/MS flag)

$U$ - Analyte analyzed for but undetected. Analyte result was below the contract required quantitation limit (CRQL).

\section{UNCLASSIFIED}




\section{UNCLASSIFIED}

LIMS 03.08.048

03/30/2010 08:53

Project: L MS MPO SCHUH SVOA

Analytical Chemistry organization (Quality Services)

P.0, Box 2009 oak Ridge, TN 378318244 (865) 574-2284

Lab Smp1 Id: A100690178

official Report

Cust Smp1 Id: H2O 100 NEW

Customer: L P BURNETT Bldg 9995 MS-8189 (865) 576-9141

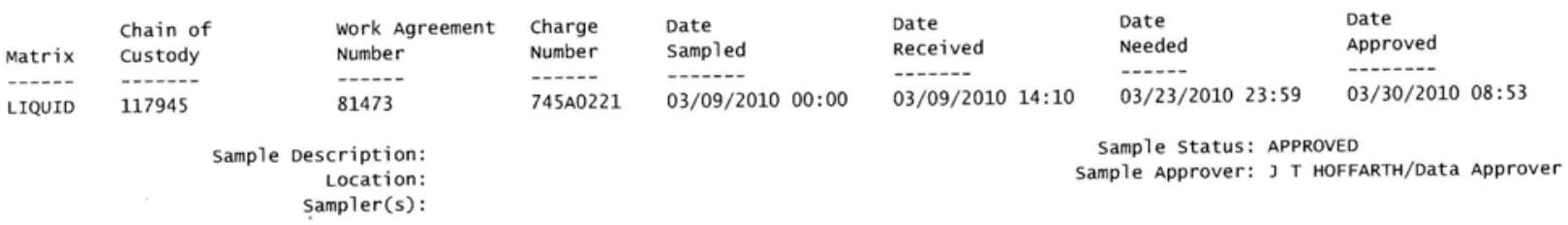

Customer comments:

Lab Comments:

Comment objects:

$\ll<<$ Organic $\gg \gg \gg>$ Test: SVOA8270 (Semi-Volatile organics by GC/MS)

Rpt Basis: AR Test Req Cnt: 01

Analysis Method: SW846 8270C (Y50-AC-65-7316)

Approver: J T HOFFARTH/Data Approver

QC Batch/File: QC10089005/5329/53810
Prep Method/Date: Sw846 3580A 03/16/2010 15:00 Test Status: APPROVED

HT Deadline: 04/25/2010 23:59

Date Analyzed: 03/25/2010 20:16 Lab Group: OYGCMS

\begin{tabular}{|c|c|}
\hline Analyte Id & Analyte Name \\
\hline 120821 & 1,2,4-Trichlorobenzene \\
\hline 95501 & 1,2-Dichlorobenzene \\
\hline 122667 & 1,2-Diphenylhydrazine \\
\hline 541731 & 1,3-Dichlorobenzene \\
\hline 106467 & 1,4-Dichlorobenzene \\
\hline 95954 & $2,4,5$-Trichlorophenol \\
\hline 88062 & 2,4,6-Trichloropheno 1 \\
\hline 120832 & 2,4-Dichlorophenol \\
\hline 105679 & 2,4-Dimethylphenol \\
\hline 51285 & 2,4-Dinitrophenol \\
\hline 121142 & 2,4-Dinitrotoluene \\
\hline 606202 & 2,6-Dinitrotoluene \\
\hline 91587 & 2-Chloronaphthalene \\
\hline 95578 & 2-Ch7orophenol \\
\hline 534521 & 2-Methyl-4,6-dinitrophenol \\
\hline 91576 & 2-Methylnaphthalene \\
\hline 95487 & 2-Methylphenol \\
\hline 88744 & 2-Nitrobenzenamine \\
\hline 88755 & 2-Nitrophenol \\
\hline 91941 & $3,3^{\circ}$-Dichlorobenzidine \\
\hline N2799 & 3- and 4- Methylphenol \\
\hline 99092 & 3-Nitrobenzenamine \\
\hline 101553 & 4-Bromopheny 1 pheny 1 ether \\
\hline 59507 & 4-chloro-3-methylphenol \\
\hline 106478 & 4-Chlorobenzenamine \\
\hline 7005723 & 4-Chloropheny 1pheny1 ether \\
\hline 100016 & 4-Nitrobenzenamine \\
\hline 100027 & 4-Nitrophenol \\
\hline 83329 & Acenaphthene \\
\hline 208968 & Acenaphthylene \\
\hline 62533 & Aniline \\
\hline 120127 & Anthracene \\
\hline 100516 & Benzeneme thanol \\
\hline 92875 & Benzidine \\
\hline 56553 & Benzo (a)anthracene \\
\hline 50328 & Benzo(a)pyrene \\
\hline 205992 & Benzo(b)fluoranthene \\
\hline 191242 & Benzo(ghi)perylene \\
\hline 207089 & Benzo $(k)$ fluoranthene \\
\hline 65850 & Benzoic acid \\
\hline 111911 & Bis (2-chloroethoxy)methane \\
\hline 111444 & Bis (2-chloroethy1) ether \\
\hline 108601 & Bis (2-chloroisopropy 1 ) ether \\
\hline 117817 & Bis (2-ethylhexy1)phthalate \\
\hline 85687 & Butylbenzylphthalate \\
\hline 86748 & Carbazole \\
\hline 218019 & Chrysene \\
\hline 84742 & Di-n-butylphthalate \\
\hline 117840 & Di-n-octylphthalate \\
\hline 53703 & Dibenz $(a, h)$ anthracene \\
\hline
\end{tabular}

\begin{tabular}{|c|c|c|c|c|c|c|c|c|}
\hline & stome & & Dilution & Lower & & EPA & & \\
\hline HT & Lim & $\mathrm{Fn}$ & Factor & Limit & Result & Qua1 & Confidence & Unit \\
\hline-- & $\cdots$ & -- & -N- & -..-- & 500 & u & -.......... & $\mathrm{ug} / \mathrm{L}$ \\
\hline & & & $\begin{array}{l}1 \\
1\end{array}$ & $\begin{array}{l}99 \\
110\end{array}$ & 500 & u & & $\begin{array}{l}\mathrm{ug} / \mathrm{L} \\
\mathrm{ug} / \mathrm{L}\end{array}$ \\
\hline & & & 1 & 59 & 500 & $u$ & & $\mathrm{ug} / \mathrm{L}$ \\
\hline & & & 1 & 170 & 500 & u & & $\mathrm{ug} / \mathrm{L}$ \\
\hline & & & 1 & 180 & 500 & u & & $u g / L$ \\
\hline & & & 1 & 190 & 500 & u & & $u g / L$ \\
\hline & & & 1 & 100 & 500 & u & & $u g / L$ \\
\hline & & & 1 & 98 & 500 & u & & $u g / L$ \\
\hline & & & 1 & 99 & 500 & u & & $u g / L$ \\
\hline & & & 1 & 140 & 1000 & u & & $u g / L$ \\
\hline & & & 1 & 130 & 500 & u & & $u g / L$ \\
\hline & & & 1 & 97 & 500 & u & & $u g / L$ \\
\hline & & & 1 & 87 & 500 & $u$ & & $\mathrm{ug} / \mathrm{L}$ \\
\hline & & & 1 & 100 & 500 & u & & $\mathrm{ug} / \mathrm{L}$ \\
\hline & & & 1 & 110 & 1000 & $u$ & & $\mathrm{ug} / \mathrm{L}$ \\
\hline & & & 1 & 66 & 500 & u & & $\mathrm{ug} / \mathrm{L}$ \\
\hline & & & 1 & 98 & 500 & $\mathrm{u}$ & & $u g / L$ \\
\hline & & & 1 & 110 & 500 & $u$ & & $u g / L$ \\
\hline & & & 1 & 81 & 500 & $\mathrm{u}$ & & $\mathrm{ug} / \mathrm{L}$ \\
\hline & & & 1 & 49 & 1000 & u & & $\mathrm{ug} / \mathrm{L}$ \\
\hline & & & 1 & 120 & 1000 & u & & $\mathrm{ug} / \mathrm{L}$ \\
\hline & & & 1 & 220 & 500 & $\begin{array}{l}u \\
u\end{array}$ & & $\begin{array}{l}u g / L \\
u g / L\end{array}$ \\
\hline & & & 1 & $\begin{array}{l}66 \\
55\end{array}$ & $\begin{array}{l}500 \\
500\end{array}$ & u & & $\begin{array}{l}u g / L \\
u g / L\end{array}$ \\
\hline & & & $\begin{array}{l}1 \\
1\end{array}$ & 100 & $\begin{array}{l}500 \\
500\end{array}$ & u & & $\mathrm{ug} / \mathrm{L}$ \\
\hline & & & 1 & 67 & 500 & $u$ & & $\mathrm{ug} / \mathrm{L}$ \\
\hline & & & 1 & 110 & 1000 & $u$ & & $u g / L$ \\
\hline & & & 1 & 100 & 1000 & $u$ & & $u g / L$ \\
\hline & & & 1 & 91 & 500 & u & & $\mathrm{ug} / \mathrm{L}$ \\
\hline & & & 1 & 100 & 500 & u & & $u g / L$ \\
\hline & & & 1 & 40 & 1000 & u & & $\mathrm{ug} / \mathrm{L}$ \\
\hline & & & 1 & 52 & 500 & $u$ & & $u g / L$ \\
\hline & & & 1 & 61 & 1000 & $u$ & & ug/L \\
\hline & & & 1 & 120 & 1000 & u & & $\mathrm{ug} / \mathrm{L}$ \\
\hline & & & 1 & 57 & 500 & u & & $\mathrm{ug} / \mathrm{L}$ \\
\hline & & & 1 & 45 & 500 & $u$ & & $u g / L$ \\
\hline & & & 1 & 110 & 500 & u & & $\mathrm{ug} / \mathrm{L}$ \\
\hline & & & 1 & 150 & 500 & $u$ & & $u g / L$ \\
\hline & & & 1 & 150 & 500 & $u$ & & $\mathrm{ug} / \mathrm{L}$ \\
\hline & & & 1 & 190 & 1000 & $u$ & & $u g / L$ \\
\hline & & & 1 & 53 & 500 & $u$ & & $\mathrm{ug} / \mathrm{L}$ \\
\hline & & & 1 & 140 & 500 & u & & $\mathrm{ug} / \mathrm{L}$ \\
\hline & & & 1 & 70 & 500 & $u$ & & ug/L \\
\hline & & & 1 & 120 & 500 & 0 & & $\mathrm{ug} / \mathrm{L}$ \\
\hline & & & 1 & 120 & 500 & u & & $\mathrm{ug} / \mathrm{L}$ \\
\hline & & & 1 & $\begin{array}{l}01 \\
97\end{array}$ & 500 & u & & $\begin{array}{l}u g / L \\
u g / L\end{array}$ \\
\hline & & & 1 & 69 & 500 & u & & $\mathrm{ug} / \mathrm{L}$ \\
\hline & & & 1 & 140 & 500 & $u$ & & $u g / L$ \\
\hline & & & 1 & 160 & 500 & $u$ & & $u g / L$ \\
\hline
\end{tabular}

UNCLASSIFIED 
UNCLASSIFIED

LIMS 03.08 .048

03/30/2010 08:53

132649 Dibenzofuran

84662 Diethylphtha7ate

131113 Dimethylphthalate

$206440 \quad$ Fluoranthene

$86737 \quad$ Fluorene

118741 Hexachlorobenzene

77474 Hexachlorocyclopentadiene

67721 Hexachloroethane

193395 Indeno $(1,2,3-\mathrm{cd})$ pyrene

$78591 \quad$ Isophorone

$621647 \quad$ N-Nitroso-di-n-propylamine

$62759 \quad \mathrm{~N}$-Nitrosodimethylamine

$86306 \quad \mathrm{~N}$-Nitrosodiphenylamine

$91203 \quad$ Naphthalene

$98953 \quad$ Nitrobenzene

87865 Pentachloropheno7

$85018 \quad$ Phenanthrene

108952 Pheno

129000 Pyrene

TIC Id TIC Name

000123422 2-Pentanone, 4-hydroxy-4-methyl- (CAS) \$
Analytical Chemistry Organization (Quality Services)

Page 2 of 2

P.O. Box 2009 oak Ridge, TN 378318244 (865) 574-2284

official Report

Lab Smp 1 Id: A100690178

Cust Smp1 Id: H2O $100 \mathrm{NEW}$

\begin{tabular}{|c|c|c|c|c|}
\hline 92 & 500 & U & & $\mathrm{ug} / \mathrm{L}$ \\
\hline 87 & 500 & u & & $\mathrm{ug} / \mathrm{L}$ \\
\hline 100 & 500 & u & & $\mathrm{ug} / \mathrm{L}$ \\
\hline 51 & 500 & u & & $\mathrm{ug} / \mathrm{L}$ \\
\hline 74 & 500 & u & & $\mathrm{ug} / \mathrm{L}$ \\
\hline 66 & 500 & u & & $\mathrm{ug} / \mathrm{L}$ \\
\hline 260 & 500 & u & & $\mathrm{ug} / \mathrm{L}$ \\
\hline 150 & 500 & u & & $\mathrm{ug} / \mathrm{L}$ \\
\hline 100 & 500 & U & & $\mathrm{ug} / \mathrm{L}$ \\
\hline 70 & 500 & U & & $\mathrm{ug} / \mathrm{L}$ \\
\hline 65 & 500 & u & & $\mathrm{ug} / \mathrm{L}$ \\
\hline 150 & 500 & u & & $u g / L$ \\
\hline 59 & 500 & u & & $\mathrm{ug} / \mathrm{L}$ \\
\hline 97 & 500 & u & & $\mathrm{ug} / \mathrm{L}$ \\
\hline 48 & 500 & u & & $\mathrm{ug} / \mathrm{L}$ \\
\hline 54 & 1000 & u & & $\mathrm{ug} / \mathrm{L}$ \\
\hline 62 & 500 & u & & $\mathrm{ug} / \mathrm{L}$ \\
\hline 92 & 500 & u & & $\mathrm{ug} / \mathrm{L}$ \\
\hline 110 & 500 & u & & $u g / L$ \\
\hline $\begin{array}{l}\text { Retention } \\
\text { Time }\end{array}$ & Unit & $\begin{array}{l}\text { Estimated } \\
\text { Conc }\end{array}$ & $\begin{array}{l}\text { EPA } \\
\text { Qua } 7\end{array}$ & Unit \\
\hline 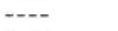 & $\ldots$ & -....- & -... & - \\
\hline 6.67 & $\min$ & 6800 & J & $\mathrm{ug} / \mathrm{L}$ \\
\hline 7.41 & $\min$ & 1200 & JN & $\mathrm{ug} / \mathrm{L}$ \\
\hline
\end{tabular}

Sample Test Comments: Target Compound(s) Initial Calibration exceeding 15\% RSD:

$\begin{array}{ll}62-53-3 & \text { Aniline } \\ 207-08-9 & \text { Benzo }[\mathrm{k}] \text { fluoranthene } \\ \text { The mean Initial Calibration } & \text { RSD }=8.7\end{array}$

Comment objects:

EPA Qualifiers:

J - Estimated value.

$\mathrm{N}$ - Presumptive evidence of a compound. (GC/MS flag)

$U$ - Analyte analyzed for but undetected. Analyte result was below the contract required quantitation limit (CRQL). 
UNCLASSIFIED

LIMS 03.08.048

03/30/2010 08:53

Analytical Chemistry organization (Quality Services)

Page 1 of 2

P.o. Box 2009 oak Ridge, TN 378318244 (865) 574-2284

A100690177

official Report

Cust Smp1 Id: H2O $50 \mathrm{NEW}$

Project: L MS MPO SCHUH SVOA

Proj Mgr: L P BURNETT (865) 576-9141

Customer: L P BURNETT Bldg 9995 MS-8189 (865) 576-9141

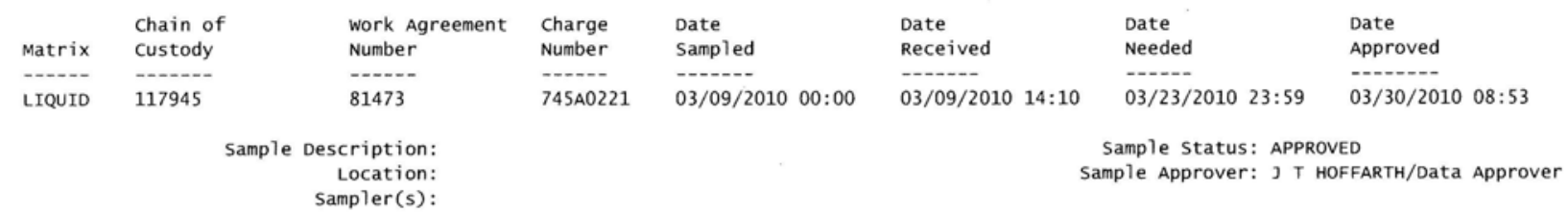

Customer comments:

Lab comments:

Comment objects:

$\ll<<$ Organic $\gg \gg>>$ Test: SVOA8270 (Semi-Volatile organics by GC/MS)

Rpt Basis: AR Test Req Cnt: 01

Analysis Method: SW846 8270C (Y50-AC-65-7316)

Approver: J T HOFFARTH/Data Approver

QC Batch/File: QC10089005/5329/53810
Prep Method/Date: SW846 3580A 03/16/2010 15:00 Test Status: APPROVED

HT Deadline: 04/25/2010 23:59 Date Analyzed: 03/25/2010 19:30 Lab Group: OYGCMS

\begin{tabular}{|c|c|}
\hline Analyte Id & Analyte Name \\
\hline -120821 & (1)- \\
\hline 120821 & 1,2,4-Trichlorobenzene \\
\hline 95501 & 1,2-Dichlorobenzene \\
\hline 122667 & 1,2-Diphenylhydrazine \\
\hline 541731 & 1,3-Dichlorobenzene \\
\hline 106467 & 1,4-Dichlorobenzene \\
\hline 95954 & 2,4,5-Trichlorophenol \\
\hline 88062 & 2,4,6-Trichlorophenol \\
\hline 120832 & 2,4-Dichlorophenol \\
\hline 105679 & 2,4-Dimethylphenol \\
\hline 51285 & 2,4-Dinitrophenol \\
\hline 121142 & 2,4-Dinitrotoluene \\
\hline 606202 & 2,6-Dinitrotoluene \\
\hline 91587 & 2-Chloronaphthalene \\
\hline 95578 & 2-Chlorophenol \\
\hline 534521 & 2-Methy 1-4,6-dinitropheno 1 \\
\hline 91576 & 2-Methylnaphthalene \\
\hline 95487 & 2-Methylphenol \\
\hline 88744 & 2-Nitrobenzenamine \\
\hline 88755 & 2-Nitrophenol \\
\hline 91941 & 3, ' - Dichlorobenzidine \\
\hline N2799 & 3- and 4- Methylphenol \\
\hline 99092 & 3-Nitrobenzenamine \\
\hline 101553 & 4-Bromopheny 1 pheny 1 ether \\
\hline 59507 & 4-Ch1oro-3-methylphenol \\
\hline 106478 & 4-Chlorobenzenamine \\
\hline 7005723 & 4-Ch1oropheny 1 pheny 1 ether \\
\hline 100016 & 4-Nitrobenzenamine \\
\hline 100027 & 4-Nitrophenol \\
\hline 83329 & Acenaphthene \\
\hline 208968 & Acenaphthylene \\
\hline 62533 & Aniline \\
\hline 120127 & Anthracene \\
\hline 100516 & Benzeneme thanol \\
\hline 92875 & Benzidine \\
\hline 56553 & Benzo (a) anthracene \\
\hline 50328 & Benzo(a)pyrene \\
\hline 205992 & Benzo(b)fluoranthene \\
\hline 191242 & Benzo(ghi)perylene \\
\hline 207089 & Benzo(k)fluoranthene \\
\hline 65850 & Benzoic acid \\
\hline 111911 & Bis (2-chloroethoxy)methane \\
\hline 111444 & Bis (2-chloroethyl) ether \\
\hline 108601 & Bis (2-chloroisopropy 1 ) ether \\
\hline 117817 & Bis(2-ethylhexy1)phthalate \\
\hline 85687 & Butylbenzylphthalate \\
\hline 86748 & Carbazole \\
\hline 218019 & Chrysene \\
\hline 84742 & Di-n-butylphthalate \\
\hline 117840 & Di-n-octylphthalate \\
\hline 53703 & Dibenz $(\mathrm{a}, \mathrm{h})$ anthracene \\
\hline
\end{tabular}

\begin{tabular}{|c|c|c|c|c|c|c|c|c|}
\hline \multicolumn{3}{|c|}{ Customer } & \multirow{2}{*}{$\begin{array}{l}\text { Dilution } \\
\text { Factor }\end{array}$} & \multirow{2}{*}{$\begin{array}{l}\text { Lower } \\
\text { Limit }\end{array}$} & \multirow[b]{2}{*}{ Result } & \multirow{2}{*}{$\begin{array}{l}\text { EPA } \\
\text { Qual }\end{array}$} & \multirow[b]{2}{*}{ Confidence } & \multirow[b]{2}{*}{ Unit } \\
\hline HT & Lim & Fn & & & & & & \\
\hline-- & --- & -- & ---- & $-\cdots$ & -..-- & --- & - & $\cdots$ \\
\hline & & & 1 & 99 & 500 & u & & $\mathrm{ug} / \mathrm{L}$ \\
\hline & & & 1 & 110 & 500 & u & & $\mathrm{ug} / \mathrm{L}$ \\
\hline & & & 1 & 59 & 500 & u & & $u g / L$ \\
\hline & & & 1 & 170 & 500 & u & & $\mathrm{ug} / \mathrm{L}$ \\
\hline & & & 1 & 180 & 500 & u & & $\mathrm{ug} / \mathrm{L}$ \\
\hline & & & 1 & 190 & 500 & u & & $u g / L$ \\
\hline & & & 1 & 100 & 500 & $u$ & & $u g / L$ \\
\hline & & & 1 & 98 & 500 & $u$ & & $u g / L$ \\
\hline & & & 1 & 99 & 500 & $\mathrm{u}$ & & $\mathrm{ug} / \mathrm{L}$ \\
\hline & & & 1 & 140 & 1000 & $u$ & & ug $/ \mathrm{L}$ \\
\hline & & & 1 & 130 & 500 & $u$ & & $\mathrm{ug} / \mathrm{L}$ \\
\hline & & & 1 & 97 & 500 & $u$ & & $u g / L$ \\
\hline & & & 1 & 87 & 500 & $\mathbf{u}$ & & $\mathrm{ug} / \mathrm{L}$ \\
\hline & & & 1 & 100 & 500 & u & & $u g / L$ \\
\hline & & & 1 & 110 & 1000 & u & & $\mathrm{ug} / \mathrm{L}$ \\
\hline & & & 1 & 66 & 500 & $\mathbf{u}$ & & $\mathrm{ug} / \mathrm{L}$ \\
\hline & & & 1 & 98 & 500 & u & & $u g / L$ \\
\hline & & & 1 & 110 & 500 & $\mathbf{u}$ & & $\mathrm{ug} / \mathrm{L}$ \\
\hline & & & 1 & 81 & 500 & u & & $u g / L$ \\
\hline & & & 1 & 49 & 1000 & u & & $\mathrm{ug} / \mathrm{L}$ \\
\hline & & & 1 & 120 & 1000 & u & & $u g / L$ \\
\hline & & & 1 & 220 & 500 & u & & $\mathrm{ug} / \mathrm{L}$ \\
\hline & & & 1 & 66 & 500 & u & & $\mathrm{ug} / \mathrm{L}$ \\
\hline & & & 1 & 55 & 500 & u & & $u g / L$ \\
\hline & & & 1 & 100 & 500 & u & & $\mathrm{ug} / \mathrm{L}$ \\
\hline & & & 1 & 67 & 500 & $u$ & & $\mathrm{ug} / \mathrm{L}$ \\
\hline & & & 1 & 110 & 1000 & $u$ & & $\mathrm{ug} / \mathrm{L}$ \\
\hline & & & 1 & 100 & 1000 & $\mathrm{u}$ & & $\mathrm{ug} / \mathrm{L}$ \\
\hline & & & 1 & 91 & 500 & $u$ & & $\mathrm{ug} / \mathrm{L}$ \\
\hline & & & 1 & 100 & 500 & u & & $\mathrm{ug} / \mathrm{L}$ \\
\hline & & & 1 & 40 & 1000 & u & & $\mathrm{ug} / \mathrm{L}$ \\
\hline & & & 1 & 52 & 500 & u & & $\mathrm{ug} / \mathrm{L}$ \\
\hline & & & 1 & 61 & 1000 & u & & $u g / L$ \\
\hline & & & 1 & 120 & 1000 & $\mathrm{u}$ & & $u g / L$ \\
\hline & & & 1 & 57 & 500 & $u$ & & $\mathrm{ug} / \mathrm{L}$ \\
\hline & & & 1 & 45 & 500 & $\mathrm{u}$ & & $u g / L$ \\
\hline & & & 1 & 110 & 500 & $\mathrm{u}$ & & $u g / L$ \\
\hline & & & 1 & 150 & 500 & $u$ & & $u g / L$ \\
\hline & & & 1 & 150 & 500 & u & & $\mathrm{ug} / \mathrm{L}$ \\
\hline & & & 1 & 190 & 1000 & u & & $\mathrm{ug} / \mathrm{L}$ \\
\hline & & & 1 & 53 & 500 & U & & $\mathrm{ug} / \mathrm{L}$ \\
\hline & & & 1 & 140 & 500 & U & & $\mathrm{ug} / \mathrm{L}$ \\
\hline & & & 1 & 70 & 500 & u & & $\mathrm{ug} / \mathrm{L}$ \\
\hline & & & 1 & 120 & 500 & u & & $\mathrm{ug} / \mathrm{L}$ \\
\hline & & & 1 & 120 & 500 & u & & $u g / L$ \\
\hline & & & 1 & 61 & 500 & u & & $\mathrm{ug} / \mathrm{L}$ \\
\hline & & & 1 & 97 & 500 & U & & $u g / L$ \\
\hline & & & 1 & 69 & 500 & U & & $\mathrm{ug} / \mathrm{L}$ \\
\hline & & & 1 & 140 & 500 & $\begin{array}{l}u \\
u\end{array}$ & & $u g / L$ \\
\hline
\end{tabular}

UNCLASSIFIED 
UNCLASSIFIED

LIMS 03.08 .048

03/30/2010 08:53

132649 Dibenzofura

Diethylphthalate

131113 Dimethylphthalate

$206440 \quad$ Fluoranthene

86737 Fluorene

118741 Hexachlorobenzene

77474 Hexachlorocyclopentadiene

67721 Hexachloroethane

193395 Indeno(1,2,3-cd)pyrene

78591 Isophorone

$621647 \quad \mathrm{~N}$-Nitroso-di-n-propylamine

$62759 \quad \mathrm{~N}$-Nitrosodimethylamine

$86306 \quad \mathrm{~N}$-Nitrosodiphenylamine

91203 Naphthalene

98953 Nitrobenzene

87865 Pentachlorophenol

$85018 \quad$ Phenanthrene

108952 Phenol

129000 Pyrene

\begin{tabular}{ll} 
TIC Id & TIC Name \\
\hline 000123422 & $\begin{array}{l}2 \text {-Pentanone, 4-hydroxy-4-methy 1- (CAS) S } \\
\text { unknown } \\
\text { unknown } \\
\text { unknown }\end{array}$
\end{tabular}

Analytical Chemistry organization (Quality Services) P.O. Box 2009 oak Ridge, TN 378318244 (865) 574-2284 official Report

1
1
1
1
1
1
1
1
1
1
1
1
1
1
1
1
1
1
1

\section{$\mathrm{Fn}$}

$\begin{array}{lll}1 & 92 & 500 \\ 1 & 87 & 500 \\ 1 & 100 & 500 \\ 1 & 51 & 500 \\ 1 & 74 & 500 \\ 1 & 66 & 500 \\ 1 & 260 & 500 \\ 1 & 150 & 500 \\ 1 & 100 & 500 \\ 1 & 70 & 500 \\ 1 & 65 & 500 \\ 1 & 150 & 500 \\ 1 & 59 & 500 \\ 1 & 97 & 500 \\ 1 & 48 & 500 \\ 1 & 54 & 1000 \\ 1 & 62 & 500 \\ 1 & 92 & 500 \\ 1 & 110 & 500 \\ & & \\ & \text { Retention } & \\ & \text { Time } & \text { Uni } \\ & ---- & --- \\ & 7.44 & \text { min } \\ & 6.77 & \text { min } \\ & 8.38 & \text { min } \\ & 8.71 & \text { min }\end{array}$

Page 2 of 2 Lab Smpl Id: A100690177 cust Smp1 Id: H2O $50 \mathrm{NEW}$

$u$ ug/L

U u ug/L

$500 \quad u \quad u g / L$

$500-4$

u $\quad$ ug/L

$500 \quad u \quad u g / L$

$500 \quad u \quad u g / L$

$u g / L$

$u g / L$

$\mathrm{ug} / \mathrm{L}$

$u g / L$

$\mathrm{ug} / \mathrm{L}$

ug/L

ug/L

$\mathrm{ug} / \mathrm{L}$

$\begin{array}{llll} & \text { Estimated } & \text { EPA } & \\ \text { Unit } & \text { Conc } & \text { Qual } & \text { Unit } \\ ------ & --- & -\cdot \\ \min & 1700 & \mathrm{JN} & \mathrm{ug} / \mathrm{L} \\ \min & 19000 & \mathrm{~J} & \mathrm{ug} / \mathrm{L} \\ \min & 370 & \mathrm{~J} & \mathrm{ug} / \mathrm{L} \\ \min & 950 & \mathrm{~J} & \mathrm{ug} / \mathrm{L}\end{array}$

Sample Test comments: Target Compound(s) Initial Calibration exceeding 15\% RSD:

$$
\begin{array}{ll}
62-53-3 & \text { Aniline } \\
207-08-9 & \text { Benzo }[\mathrm{k}] \text { fluoranthene }
\end{array}
$$

The mean Initial Calibration RSD $=8.7$

Comment objects:

EPA Qualifiers:

J - Estimated value.

N - Presumptive evidence of a compound. (GC/MS flag)

$U$ - Analyte analyzed for but undetected. Analyte result was below the contract required quantitation limit (CRQL).

\section{UNCLASSIFIED}


UNCLASSIFIED

Analytical Chemistry organization (Quality Services)

Page 1 of 2

LIMS 03.08 .048

P.o. Box 2009 oak Ridge, TN 378318244 (865) 574-2284

A100550085

03/12/2010 16:04

official Report

Cust Smp1 Id: 300 mrad Nitric

Project: L MS MPO SCHUH SVOA

PROj Mgr: L P BURNETT (865) 576-9141

Customer: L P BURNETT B1dg 9995 MS-8189 (865) 576-9141

\begin{tabular}{|c|c|c|c|c|c|c|c|}
\hline Matrix & $\begin{array}{l}\text { Chain of } \\
\text { Custody }\end{array}$ & $\begin{array}{l}\text { Work Agreement } \\
\text { Number }\end{array}$ & $\begin{array}{l}\text { Charge } \\
\text { Number }\end{array}$ & $\begin{array}{l}\text { Date } \\
\text { Sampled }\end{array}$ & $\begin{array}{l}\text { Date } \\
\text { Received }\end{array}$ & $\begin{array}{l}\text { Date } \\
\text { Needed }\end{array}$ & $\begin{array}{l}\text { Date } \\
\text { Approved }\end{array}$ \\
\hline ----- & $-\cdots-$ & --.-- & $\cdots-$ & ---1-- & --n-- & ----- & - \\
\hline LIQUID & 117936 & 81473 & $745 A 0221$ & 02/23/2010 00:00 & $02 / 23 / 2010 \quad 14: 30$ & 03/09/2010 23:59 & $03 / 12 / 201016: 04$ \\
\hline
\end{tabular}

Sample Description: $0.5 \mathrm{M}$ HNO3

Sample Status: APPROVED

Location:

Sample Approver: 3 T HOFFARTH/Data Approver

Customer comments:

Lab Comments:

Comment objects:

$\ll<<$ organic $\gg \gg>>$

Rpt Basis: AR

Test: SVOA8270 (Semi-Volatile organics by GC/MS) AR

Analysis Method: SW846 $8270 \mathrm{C}$ (Y50-AC-65-7316)

Approver: J T HOFFARTH/Data Approver

QC Batch/File: QC10070010/5314/53787
Prep Method/Date: SW846 3580A 02/24/2010 13:00 Test Status: APPROVED

HT Deadline: 04/05/2010 23:59 Date Analyzed: 03/10/2010 20:11

Lab Group: OYGCMS

\begin{tabular}{|c|c|}
\hline Analyte Id & Analyte Name \\
\hline 120821 & $1,2,4$-Trichlorobenzene \\
\hline 95501 & 1,2-Dichlorobenzene \\
\hline 122667 & 1,2-Diphenylhydrazine \\
\hline 541731 & 1,3-Dichlorobenzene \\
\hline 106467 & 1,4-Dich7orobenzene \\
\hline 95954 & 2,4,5-Trichlorophenol \\
\hline 88062 & 2,4,6-Trichloropheno 1 \\
\hline 120832 & 2,4-Dichlorophenol \\
\hline 105679 & 2,4-Dimethylphenol \\
\hline 51285 & 2,4-Dinit rophenol \\
\hline 121142 & 2,4-Dinitrotoluene \\
\hline 606202 & 2,6-Dinitrotoluene \\
\hline 91587 & 2-Chloronaphthalene \\
\hline 95578 & 2-Chlorophenol \\
\hline 534521 & 2-Methyl-4,6-dinitrophenol \\
\hline 91576 & 2-Methylnaphthalene \\
\hline 95487 & 2-Methylphenol \\
\hline 88744 & 2-Nitrobenzenamine \\
\hline 88755 & 2-Nitrophenol \\
\hline 91941 & $3,3^{\prime}$-Dichlorobenzidine \\
\hline N2799 & 3- and 4- Methylphenol \\
\hline 99092 & 3-Nitrobenzenamine \\
\hline 101553 & 4-Bromopheny 1 pheny 1 ether \\
\hline 59507 & 4-Chloro-3-methylphenol \\
\hline 106478 & 4-Chlorobenzenamine \\
\hline 7005723 & 4-Ch 1 oropheny 1 pheny 1 ether \\
\hline 100016 & 4-Nitrobenzenamine \\
\hline 100027 & 4-Nitrophenol \\
\hline 83329 & Acenaphthene \\
\hline 208968 & Acenaphthylene \\
\hline 62533 & Aniline \\
\hline 120127 & Anthracene \\
\hline 100516 & Benzeneme thanol \\
\hline 92875 & Benzidine \\
\hline 56553 & Benzo (a) anthracene \\
\hline 50328 & Benzo(a)pyrene \\
\hline 205992 & Benzo(b)fluoranthene \\
\hline 191242 & Benzo(ghi)perylene \\
\hline 207089 & Benzo (k)fluoranthene \\
\hline 65850 & Benzoic acid \\
\hline 111911 & Bis(2-chloroethoxy)methane \\
\hline 111444 & Bis (2-chloroethyl) ether \\
\hline 108601 & Bis (2-chloroisopropy1) ether \\
\hline 117817 & Bis (2-ethylhexy1)phthalate \\
\hline 85687 & Butylbenzylphthalate \\
\hline 86748 & Carbazole \\
\hline 218019 & Chrysene \\
\hline 84742 & Di-n-butylphthalate \\
\hline 117840 & Di-n-octylphthalate \\
\hline 53703 & Dibenz $(a, h)$ anthracene \\
\hline
\end{tabular}

\begin{tabular}{|c|c|c|c|c|c|c|c|c|}
\hline \multicolumn{3}{|c|}{ Customer } & \multirow{2}{*}{$\begin{array}{l}\text { Dilution } \\
\text { Factor }\end{array}$} & \multirow{2}{*}{$\begin{array}{l}\text { Lower } \\
\text { Limit }\end{array}$} & \multirow[b]{2}{*}{ Result } & \multirow{2}{*}{$\begin{array}{l}\text { EPA } \\
\text { Qual }\end{array}$} & \multirow[b]{2}{*}{ Confidence } & \multirow[b]{2}{*}{ Unit } \\
\hline HT & Lim & $\mathrm{Fn}$ & & & & & & \\
\hline & -- & -- & $-\cdots-$ & ---- & -...... & $-\cdot$ & - & - \\
\hline & & & 1 & 99 & 500 & u & & $u g / L$ \\
\hline & & & 1 & 110 & 500 & $u$ & & $\mathrm{ug} / \mathrm{L}$ \\
\hline & & & 1 & 59 & 500 & $u$ & & $\mathrm{ug} / \mathrm{L}$ \\
\hline & & & 1 & 170 & 500 & u & & $u g / L$ \\
\hline & & & 1 & 180 & 500 & u & & ug/L \\
\hline & & & 1 & 190 & 500 & u & & $u g / L$ \\
\hline & & & 1 & 100 & 500 & u & & $\mathrm{ug} / \mathrm{L}$ \\
\hline & & & 1 & 98 & 500 & u & & ug/L \\
\hline & & & 1 & 99 & 500 & u & & $\mathrm{ug} / \mathrm{L}$ \\
\hline & & & 1 & 140 & 1000 & u & & ug/L \\
\hline & & & 1 & 130 & 500 & u & & $\mathrm{ug} / \mathrm{L}$ \\
\hline & & & 1 & 97 & 500 & $\mathrm{u}$ & & $\mathrm{ug} / \mathrm{L}$ \\
\hline & & & 1 & 87 & 500 & $u$ & & $\mathrm{ug} / \mathrm{L}$ \\
\hline & & & 1 & 100 & $\begin{array}{l}500 \\
1000\end{array}$ & u & & $u g / L$ \\
\hline & & & 1 & 110 & 500 & u & & $\begin{array}{l}u g / L \\
u g / L\end{array}$ \\
\hline & & & 1 & 98 & 500 & u & & $\mathrm{ug} / \mathrm{L}$ \\
\hline & & & $\begin{array}{l}1 \\
1\end{array}$ & 110 & 500 & u & & $\mathrm{ug} / \mathrm{L}$ \\
\hline & & & 1 & 81 & 500 & u & & $\mathrm{ug} / \mathrm{L}$ \\
\hline & & & 1 & 49 & 1000 & u & & ug/L \\
\hline & & & 1 & 120 & 1000 & u & & ug/L \\
\hline & & & 1 & 220 & 500 & $u$ & & $u g / L$ \\
\hline & & & 1 & 66 & 500 & $u$ & & $\mathrm{ug} / \mathrm{L}$ \\
\hline & & & 1 & 55 & 500 & u & & $\mathrm{ug} / \mathrm{L}$ \\
\hline & & & 1 & 100 & 500 & u & & $\mathrm{ug} / \mathrm{L}$ \\
\hline & & & 1 & 67 & 500 & U & & $u g / L$ \\
\hline & & & 1 & 110 & 1000 & $u$ & & $\mathrm{ug} / \mathrm{L}$ \\
\hline & & & 1 & 100 & 1000 & 0 & & $\mathrm{ug} / \mathrm{L}$ \\
\hline & & & 1 & 91 & 500 & 0 & & $\mathrm{ug} / \mathrm{L}$ \\
\hline & & & 1 & 100 & $\begin{array}{l}500 \\
1000\end{array}$ & 4 & & ug/L \\
\hline & & & 1 & $\begin{array}{l}40 \\
52\end{array}$ & 500 & u & & ug/L \\
\hline & & & 1 & 61 & 1000 & u & & ug/L \\
\hline & & & 1 & 120 & 1000 & $u$ & & $\mathrm{ug} / \mathrm{L}$ \\
\hline & & & 1 & 57 & 500 & $u$ & & $\mathrm{ug} / \mathrm{L}$ \\
\hline & & & 1 & 45 & 500 & u & & $u g / L$ \\
\hline & & & 1 & 110 & 500 & u & & $\mathrm{ug} / \mathrm{L}$ \\
\hline & & & 1 & 150 & 500 & u & & $u g / L$ \\
\hline & & & 1 & 150 & 500 & u & & $u g / L$ \\
\hline & & & 1 & 190 & 1000 & u & & $u g / L$ \\
\hline & & & 1 & 53 & 500 & u & & $u g / L$ \\
\hline & & & 1 & 140 & 500 & u & & $\mathrm{ug} / \mathrm{L}$ \\
\hline & & & 1 & 70 & 500 & u & & $\mathrm{ug} / \mathrm{L}$ \\
\hline & & & 1 & 120 & 500 & u & & $\mathrm{ug} / \mathrm{L}$ \\
\hline & & & 1 & 120 & 500 & 0 & & $\mathrm{ug} / \mathrm{L}$ \\
\hline & & & 1 & 61 & 500 & 0 & & $\mathrm{ug} / \mathrm{L}$ \\
\hline & & & 1 & 97 & 500 & u & & ug/L \\
\hline & & & 1 & 140 & 500 & u & & ug/L \\
\hline & & & 1 & 160 & 500 & $u$ & & $u g / L$ \\
\hline
\end{tabular}

UNCLASSIFIED 


\section{UNCLASSIFIED}

LIMS 03.08.048

03/12/2010 16:04

132649 Dibenzofuran

84662 Diethylphthalate

131113 Dimethylphthalate

$206440 \quad$ Fluoranthene

86737 Fluorene

118741 Hexachlorobenzene

77474 Hexachlorocyclopentadiene

67721 Hexachloroethane

193395 Indeno(1, 2,3-cd)pyrene

78591 Isophorone

$621647 \quad \mathrm{~N}$-Nitroso-di-n-propylamine

$62759 \quad \mathrm{~N}$-Nitrosodimethylamine

$86306 \quad \mathrm{~N}$-Nitrosodiphenylamine

98953

87865

85018

108952

Naphthalene

Nitrobenzene

Pentachlorophenol

Phenanthrene

Phenol

129000 Pyrene

TIC Id

000123422

044970050

\section{TIC Name}

2-Pentanone, 4-hydroxy-4-methyl- (CAS) \$

Pentanoic acid, 2,2-dimethyl-, ethenyl e

unknown

unknown
Analytical Chemistry organization (Quality Services) P.o. Box 2009 Oak Ridge, TN 378318244 (865) 574-2284 official Report

$\begin{array}{ll}1 & 9 \\ 1 & 87 \\ 1 & 100 \\ 1 & 5 \\ 1 & 7 \\ 1 & 66 \\ 1 & 2 \\ 1 & 150 \\ 1 & 100 \\ 1 & 70 \\ 1 & 65 \\ 1 & 150 \\ 1 & 59 \\ 1 & 97 \\ 1 & 48 \\ 1 & 54 \\ 1 & 62 \\ 1 & 92 \\ 1 & 11\end{array}$

Fn

$\begin{array}{ll}\text { Retention } & \\ - \text { Time } & \text { Unit } \\ ---- & --- \\ 7.47 & \text { min } \\ 8.75 & \text { min } \\ 6.84 & \text { min } \\ 8.47 & \text { min } \\ 8.79 & \text { min }\end{array}$

Page 2 of 2

Lab Smpl Id: A100550085

Cust Smpl Id: 300 Mrad Nitric

\begin{tabular}{|c|c|c|c|c|}
\hline 2 & 500 & $u$ & & $u g / L$ \\
\hline 7 & 500 & $u$ & & $\mathrm{ug} / \mathrm{L}$ \\
\hline 00 & 500 & u & & $u g / L$ \\
\hline 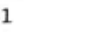 & 500 & $u$ & & $u g / L$ \\
\hline & 500 & $u$ & & ug/ \\
\hline & 500 & u & & $\mathrm{ug} /$ \\
\hline 0 & 500 & u & & $\mathrm{ug} / \mathrm{L}$ \\
\hline 50 & 500 & u & & ug/ \\
\hline 00 & 500 & u & & ug/ \\
\hline & 500 & $u$ & & ug/ \\
\hline & 500 & u & & ug/ \\
\hline 0 & 500 & u & & ug/ \\
\hline & 500 & $u$ & & ug/ \\
\hline & 500 & $u$ & & ug/ \\
\hline & 500 & $u$ & & ug/ \\
\hline & 1000 & u & & $u g / L$ \\
\hline & 500 & u & & $\mathrm{ug} / \mathrm{L}$ \\
\hline & 500 & u & & $\mathrm{ug} / \mathrm{L}$ \\
\hline & 500 & $u$ & & ug/L \\
\hline tention & & Estimated & EPA & \\
\hline me & Unit & Conc & Qual & Uni \\
\hline & ---- & --n-- & --- & \\
\hline 47 & $\min$ & 2200 & JN & \\
\hline 75 & $\min$ & 370 & JN & $u g / L$ \\
\hline & $\min$ & 23000 & J & $u g / L$ \\
\hline 7 & $\min$ & 230 & J & $u g / L$ \\
\hline & $\min$ & 980 & J & ug/ \\
\hline
\end{tabular}

Sample Test comments: Target Compound(s) Initial Calibration exceeding 15\% RSD:

$62-53-3 \quad$ Aniline
$207-08-9$
The mean Initial calibration $R S D=8.7$

Comment objects:

EPA Qualifiers:

J - Estimated value.

N - Presumptive evidence of a compound. (GC/MS flag)

$U$ - Analyte analyzed for but undetected. Analyte result was below the contract required quantitation limit (CRQL).

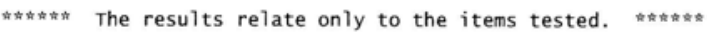

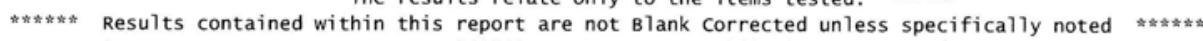

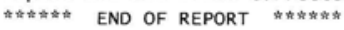

UNCLASSIFIED 
UNCLASSIFIED

LIMS 03.08 .048

03/30/2010 08:53

Analytical Chemistry Organization (Quality Services)

Page 1 of 2

P.o. Box 2009 oak Ridge, TN 378318244 (865) 574-2284

Lab Smp1 Id: A100690176

official Report

Cust Smpl Id: NITRIC 250 Mrad

Project: L MS MPO SCHUH SVOA

Proj Mgr: L P BURNETT (865) 576-9141

Customer: L. P BURNETT B1dg 9995 MS-8189 (865) 576-9141

\begin{tabular}{|c|c|c|c|c|c|c|c|}
\hline Matrix & $\begin{array}{l}\text { Chain of } \\
\text { Custody }\end{array}$ & $\begin{array}{l}\text { Work Agreement } \\
\text { Number }\end{array}$ & $\begin{array}{l}\text { Charge } \\
\text { Number }\end{array}$ & $\begin{array}{l}\text { Date } \\
\text { Sampled }\end{array}$ & $\begin{array}{l}\text { Date } \\
\text { Received }\end{array}$ & $\begin{array}{l}\text { Date } \\
\text { Needed }\end{array}$ & $\begin{array}{l}\text { Date } \\
\text { Approved }\end{array}$ \\
\hline -.... & --n-- & ---- & ----- & - & -.....- & --.-- & ---n-- \\
\hline LIQUID & 117945 & 81473 & $745 A 0221$ & $03 / 09 / 201000: 00$ & $03 / 09 / 2010 \quad 14: 10$ & $03 / 23 / 2010 \quad 23: 59$ & $03 / 30 / 2010 \quad 08: 53$ \\
\hline
\end{tabular}

Sample Description: $0.5 \mathrm{M}$ HNO3

Sample status: APPROVED

Location:

Sample Approver: J T HOFFARTH/Data Approver

Customer comments:

Lab Comments:

Comment objects:

$\ll<<$ Organic $\gg \gg>>$

Rpt Basis: AR Test Req Cnt: 01

Analysis Method: SW846 8270C (Y50-AC-65-7316)

Approver: J T HOFFARTH/Data Approver

QC Batch/File: QC10089005/5329/53810
Prep Method/Date: Sw846 3580A 03/16/2010 15:00 Test Status: APPROVED

HT Deadline: 04/25/2010 23:59

Date Analyzed: 03/25/2010 18:43

Lab Group: OYGCMS

\begin{tabular}{|c|c|}
\hline Analyte Id & Analyte Name \\
\hline ------ & 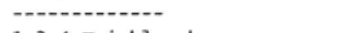 \\
\hline 120821 & 1,2,4-Trichlorobenzene \\
\hline 95501 & 1,2-Dichlorobenzene \\
\hline 122667 & 1,2-Diphenylhydrazine \\
\hline 541731 & 1,3-Dichlorobenzene \\
\hline 106467 & 1,4-Dichlorobenzene \\
\hline 95954 & 2,4,5-Trichlorophenol \\
\hline 88062 & 2,4,6-Trichlorophenol \\
\hline 120832 & 2,4-Dichlorophenol \\
\hline 105679 & 2,4-Dimethylphenol \\
\hline 51285 & 2,4-Dinitrophenol \\
\hline 121142 & 2,4-Dinitrotoluene \\
\hline 606202 & 2,6-Dinitrotoluene \\
\hline 91587 & 2-Chloronaphthalene \\
\hline 95578 & 2-Ch1orophenol \\
\hline 534521 & 2-Methy 1-4,6-dinitropheno 1 \\
\hline 91576 & 2-Methylnaphthalene \\
\hline 95487 & 2-Methylphenol \\
\hline 88744 & 2-Nitrobenzenamine \\
\hline 88755 & 2-Nitrophenol \\
\hline 91941 & $3,3^{\circ}$-Dichlorobenzidine \\
\hline N2799 & 3- and 4- Methylphenol \\
\hline 99092 & 3-Nitrobenzenamine \\
\hline 101553 & 4-8romopheny 1 pheny 1 ether \\
\hline 59507 & 4-Chloro-3-methy?pheno 1 \\
\hline 106478 & 4-Ch1orobenzenamine \\
\hline 7005723 & 4-Chloropheny 1 pheny 1 ether \\
\hline 100016 & 4-Nitrobenzenamine \\
\hline 100027 & 4-Nitrophenol \\
\hline 83329 & Acenaphthene \\
\hline 208968 & Acenaphthylene \\
\hline 62533 & Aniline \\
\hline 120127 & Anthracene \\
\hline 100516 & Benzenemethanol \\
\hline 92875 & Benzidine \\
\hline 56553 & Benzo(a)anthracene \\
\hline 50328 & Benzo(a)pyrene \\
\hline 205992 & Benzo(b)fluoranthene \\
\hline 191242 & Benzo(ghi)perylene \\
\hline 207089 & Benzo $(k)$ fluoranthene \\
\hline 65850 & Benzoic acid \\
\hline 111911 & Bis (2-chloroethoxy)methane \\
\hline 111444 & Bis (2-chloroethy1) ether \\
\hline 108601 & Bis(2-chloroisopropy1) ether \\
\hline 117817 & Bis(2-ethylhexyl)phthalate \\
\hline 85687 & Butylbenzylphthalate \\
\hline 86748 & Carbazole \\
\hline 218019 & Chrysene \\
\hline 84742 & Di-n-butylphthalate \\
\hline 117840 & Di-n-octylphthalate \\
\hline 53703 & Dibenz $(\mathrm{a}, \mathrm{h})$ anthracene \\
\hline
\end{tabular}

\begin{tabular}{|c|c|c|c|c|c|c|c|c|}
\hline \multicolumn{3}{|c|}{ Customer } & \multirow{2}{*}{$\begin{array}{l}\text { Dilution } \\
\text { Factor }\end{array}$} & \multirow{2}{*}{$\begin{array}{l}\text { Lower } \\
\text { Limit }\end{array}$} & \multirow[b]{2}{*}{ Result } & \multirow{2}{*}{$\begin{array}{l}\text { EPA } \\
\text { Qua1 }\end{array}$} & \multirow[b]{2}{*}{ Confidence } & \multirow[b]{2}{*}{ Unit } \\
\hline HT & Lim & $\mathrm{Fn}$ & & & & & & \\
\hline-- & $\cdots$ & -- & -....- & $-\cdots$ & ----- &.-- & - & $\cdots$ \\
\hline & & & 1 & 99 & 500 & $u$ & & $u g / L$ \\
\hline & & & 1 & 110 & 500 & $u$ & & $\mathrm{ug} / \mathrm{L}$ \\
\hline & & & 1 & 59 & 500 & u & & $\mathrm{ug} / \mathrm{L}$ \\
\hline & & & 1 & 170 & 500 & u & & $\mathrm{ug} / \mathrm{L}$ \\
\hline & & & 1 & 180 & 500 & u & & $\mathrm{ug} / \mathrm{L}$ \\
\hline & & & 1 & 190 & 500 & u & & ug/L \\
\hline & & & 1 & 100 & 500 & u & & $\mathrm{ug} / \mathrm{L}$ \\
\hline & & & 1 & 98 & 500 & u & & $u g / L$ \\
\hline & & & 1 & 99 & 500 & $u$ & & ug/L \\
\hline & & & 1 & 140 & 1000 & $u$ & & ug/L \\
\hline & & & 1 & 130 & 500 & $u$ & & $\mathrm{ug} / \mathrm{L}$ \\
\hline & & & 1 & 97 & 500 & u & & $\mathrm{ug} / \mathrm{L}$ \\
\hline & & & 1 & 87 & 500 & u & & $\mathrm{ug} / \mathrm{L}$ \\
\hline & & & 1 & 100 & 500 & $u$ & & $\mathrm{ug} / \mathrm{L}$ \\
\hline & & & 1 & 110 & 1000 & u & & $\mathrm{ug} / \mathrm{L}$ \\
\hline & & & 1 & 66 & 500 & u & & $\mathrm{ug} / \mathrm{L}$ \\
\hline & & & 1 & 98 & 500 & u & & $u g / L$ \\
\hline & & & 1 & 110 & 500 & u & & $u g / L$ \\
\hline & & & 1 & 81 & 500 & u & & $u g / L$ \\
\hline & & & 1 & 49 & 1000 & u & & $\mathrm{ug} / \mathrm{L}$ \\
\hline & & & 1 & 120 & 1000 & u & & $\mathrm{ug} / \mathrm{L}$ \\
\hline & & & 1 & 220 & 500 & u & & $u g / L$ \\
\hline & & & 1 & 66 & 500 & u & & $\mathrm{ug} / \mathrm{L}$ \\
\hline & & & 1 & 55 & 500 & u & & $u g / L$ \\
\hline & & & 1 & 100 & 500 & $u$ & & $\mathrm{ug} / \mathrm{L}$ \\
\hline & & & 1 & 67 & 500 & u & & $\mathrm{ug} / \mathrm{L}$ \\
\hline & & & 1 & 110 & 1000 & u & & $u g / L$ \\
\hline & & & 1 & 100 & 1000 & u & & $\mathrm{ug} / \mathrm{L}$ \\
\hline & & & 1 & 91 & 500 & u & & $\mathrm{ug} / \mathrm{L}$ \\
\hline & & & 1 & 100 & 500 & u & & ug/L \\
\hline & & & 1 & 40 & 1000 & u & & $u g / L$ \\
\hline & & & 1 & 52 & 500 & u & & $\mathrm{ug} / \mathrm{L}$ \\
\hline & & & 1 & 61 & 1000 & u & & $u g / L$ \\
\hline & & & 1 & 120 & 1000 & u & & $\mathrm{ug} / \mathrm{L}$ \\
\hline & & & 1 & 57 & 500 & U & & $u g / L$ \\
\hline & & & 1 & 45 & 500 & u & & $u g / L$ \\
\hline & & & 1 & 110 & 500 & u & & $\mathrm{ug} / \mathrm{L}$ \\
\hline & & & 1 & 150 & 500 & u & & $\mathrm{ug} / \mathrm{L}$ \\
\hline & & & 1 & 150 & 500 & u & & $\mathrm{ug} / \mathrm{L}$ \\
\hline & & & 1 & 190 & 1000 & u & & $\mathrm{ug} / \mathrm{L}$ \\
\hline & & & 1 & 53 & 500 & u & & $u g / L$ \\
\hline & & & 1 & 140 & 500 & u & & $\mathrm{ug} / \mathrm{L}$ \\
\hline & & & 1 & 70 & 500 & u & & $u g / L$ \\
\hline & & & 1 & 120 & 500 & u & & $\mathrm{ug} / \mathrm{L}$ \\
\hline & & & 1 & 120 & 500 & 0 & & $u g / L$ \\
\hline & & & 1 & 61 & 500 & U & & $\mathrm{ug} / \mathrm{L}$ \\
\hline & & & 1 & 97 & 500 & $u$ & & ug \\
\hline & & & 1 & 69 & $\begin{array}{l}500 \\
500\end{array}$ & $\begin{array}{l}u \\
u\end{array}$ & & ug \\
\hline & & & 1 . & $\begin{array}{l}140 \\
160\end{array}$ & 500 & u & & $\mathrm{ug} / \mathrm{L}$ \\
\hline
\end{tabular}

UNCLASSIFIED 


\section{UNCLASSIFIED}

LIMS 03.08.048

03/30/2010 08:53

\begin{tabular}{|c|c|}
\hline 132649 & Dibenzofuran \\
\hline 84662 & Diethylphtha1ate \\
\hline 131113 & Dimethylphthalate \\
\hline 206440 & Fluoranthene \\
\hline 86737 & Fluorene \\
\hline 118741 & Hexachlorobenzene \\
\hline 77474 & Hexachlorocyclopentadiene \\
\hline 67721 & Hexachloroethane \\
\hline 193395 & Indeno $(1,2,3-c d)$ pyrene \\
\hline 78591 & Isophorone \\
\hline 621647 & N-Nitroso-di-n-propylamine \\
\hline 62759 & N-Nitrosodimethylamine \\
\hline 86306 & $\mathrm{~N}-\mathrm{Ni}$ trosodiphenylamine \\
\hline 91203 & Naphthalene \\
\hline 98953 & Nitrobenzene \\
\hline 87865 & Pentach 1orophenol \\
\hline 85018 & Phenanthrene \\
\hline 108952 & $\begin{array}{l}\text { Phenol } \\
\text { Pyrene }\end{array}$ \\
\hline 129000 & Pyrene \\
\hline TIC Id & TIC Name \\
\hline 000123422 & 2-Pentanone 4-hydroxy-4-methyl- \\
\hline 000123422 & $\begin{array}{l}\text { 2-Pentanone, 4-hydroxy-4-methy1-(CAS) S } \\
\text { unknown } \\
\text { unknown }\end{array}$ \\
\hline
\end{tabular}

Analytical Chemistry organization (Quality Services)

Page 2 of 2 P.0. Box 2009 oak Ridge, TN 378318244 (865) 574-2284 Lab Smpl Id: A100690176 official Report Cust Smp1 Id: NITRIC $250 \mathrm{Mrad}$

\begin{tabular}{|c|c|c|c|c|c|}
\hline 1 & 92 & 500 & u & & $\mathrm{ug} / \mathrm{L}$ \\
\hline 1 & 87 & 500 & $u$ & & $u g / L$ \\
\hline 1 & 100 & 500 & u & & $u g / L$ \\
\hline 1 & 51 & 500 & u & & $u g / L$ \\
\hline 1 & 74 & 500 & u & & $u g / L$ \\
\hline 1 & 66 & 500 & u & & $\mathrm{ug} / \mathrm{L}$ \\
\hline 1 & 260 & 500 & u & & $\mathrm{ug} / \mathrm{L}$ \\
\hline 1 & 150 & 500 & u & & $\mathrm{ug} / \mathrm{L}$ \\
\hline 1 & 100 & 500 & u & & $\mathrm{ug} / \mathrm{L}$ \\
\hline 1 & 70 & 500 & u & & $\mathrm{ug} / \mathrm{L}$ \\
\hline 1 & 65 & 500 & u & & $\mathrm{ug} / \mathrm{L}$ \\
\hline 1 & 150 & 500 & u & & $u g / L$ \\
\hline 1 & 59 & 500 & u & & $u g / L$ \\
\hline 1 & 97 & 500 & u & & $u g / L$ \\
\hline 1 & 48 & 500 & $u$ & & $\mathrm{ug} / \mathrm{L}$ \\
\hline 1 & 54 & 1000 & u & & $\mathrm{ug} / \mathrm{L}$ \\
\hline 1 & 62 & 500 & $u$ & & $\mathrm{ug} / \mathrm{L}$ \\
\hline 1 & 92 & 500 & u & & $\mathrm{ug} / \mathrm{L}$ \\
\hline 1 & 110 & 500 & u & & $\mathrm{ug} / \mathrm{L}$ \\
\hline Fn & $\begin{array}{l}\text { Retention } \\
\text { Time }\end{array}$ & Unit & $\begin{array}{l}\text { Estimated } \\
\text { Conc }\end{array}$ & $\begin{array}{l}\text { EPA } \\
\text { Qua } 7\end{array}$ & Unit \\
\hline-- & $\cdots$ & - & -...- & -- & -... \\
\hline & 7.41 & $\min$ & 1200 & JN & $u g / L$ \\
\hline & 6.73 & $\min$ & 14000 & J & $\mathrm{ug} / \mathrm{L}$ \\
\hline & 8.72 & $\min$ & 480 & J & $u g / L$ \\
\hline
\end{tabular}

Sample Test Comments: Target Compound(s) Initial Calibration exceeding 15\% RSD:

$$
\begin{aligned}
& \text { 62-53-3 Aniline } \\
& \text { 207-08-9 Benzo[k]fluoranthene } \\
& \text { The mean Initial Calibration RSD }=8.7
\end{aligned}
$$

Comment objects

EPA Qualifiers:

J - Estimated value.

N - Presumptive evidence of a compound. (GC/MS flag)

U - Analyte analyzed for but undetected. Analyte result was below the contract required quantitation limit (CRQL). 
UNCLASSIFIED

LIMS 03.08.048

04/30/2010 13:21

Analytical Chemistry organization (Quality services)

Page 1 of 2

P.o. Box 2009 oak Ridge, TN 378318244 (865) 574-2284

Lab Smp1 Id: A100910402

official Report

Cust Smpl Id: HFIR Nitric 200Mrad

Project: L MS MPO SCHUH SVOA

Proj Mgr: L P BURNETT (865) 576-9141

Customer: L. P BURNETT B1dg 9995 MS-8189 (865) 576-9141

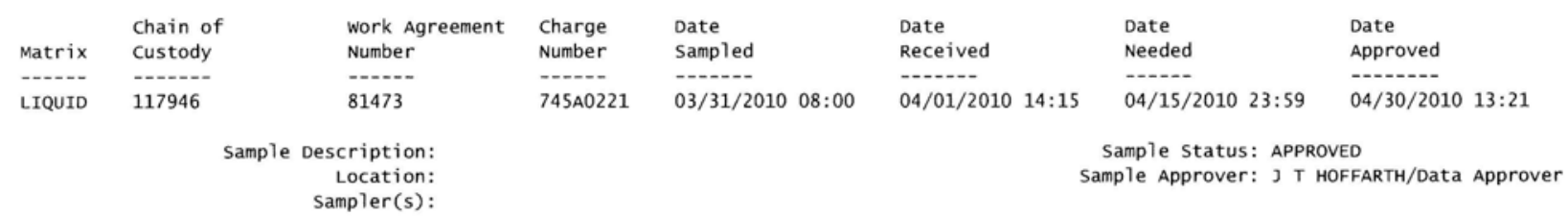

Customer comments:

Lab Comments:

Comment objects:

$\ll<$ organic $\gg \gg>$

Rpt Basis: AR Test Req Cnt: 01

Analysis Method: SW846 $8270 \mathrm{C}$ (Y50-AC-65-7316)
Approver: J T HOFFARTH/Data Approver

QC Batch/File: QC10118031/5362/53833
MS)

rep Method/Date: SW846 $3580 A$
Test Status: APPROVED

HT Deadline: 05/16/2010 23:59

Date Analyzed: 04/26/2010 22:35

Lab Group: OYGCMS

\begin{tabular}{|c|c|}
\hline Analyte Id & Analyte Name \\
\hline --n-n-n & --n-n-n- \\
\hline 120821 & 1,2,4-Trich1orobenzene \\
\hline 95501 & 1,2-Dichlorobenzene \\
\hline 122667 & 1,2-Diphenylhydrazine \\
\hline 541731 & 1,3-Dichlorobenzene \\
\hline 106467 & 1,4-Dichlorobenzene \\
\hline 95954 & $2,4,5$-Trichloropheno 1 \\
\hline 88062 & 2,4,6-Trich7orophenol \\
\hline 120832 & 2,4-Dich1oropheno 1 \\
\hline 105679 & 2,4-Dimethylphenol \\
\hline 51285 & 2,4-Dinitrophenol \\
\hline 121142 & 2,4-Dinitrotoluene \\
\hline 606202 & 2,6-Dinitrotoluene \\
\hline 91587 & 2-Chloronaphthalene \\
\hline 95578 & 2-Ch7orophenol \\
\hline 534521 & 2-Methy $7-4,6$-dinitropheno 1 \\
\hline 91576 & 2-Methylnaphthalene \\
\hline 95487 & 2-Methy 1pheno 1 \\
\hline 88744 & 2-Nit robenzenamine \\
\hline 88755 & 2-Nitrophenol \\
\hline 91941 & 3, $3^{\prime}$-Dichlorobenzidine \\
\hline N2799 & 3- and 4- Methylphenol \\
\hline 99092 & 3-Nitrobenzenamine \\
\hline 101553 & 4-Bromopheny 1 pheny 1 ether \\
\hline 59507 & 4-Chloro-3-methylphenol \\
\hline 106478 & 4-Ch7orobenzenamine \\
\hline 7005723 & 4-Ch 1 oropheny 1 pheny 1 ether \\
\hline 100016 & 4-Nitrobenzenamine \\
\hline 100027 & 4-Nitrophenol \\
\hline 83329 & Acenaphthene \\
\hline 208968 & Acenaphthylene \\
\hline 62533 & Aniline \\
\hline 120127 & Anthracene \\
\hline 100516 & Benzenemethanol \\
\hline 92875 & Benzidine \\
\hline 56553 & Benzo(a) anthracene \\
\hline 50328 & Benzo(a)pyrene \\
\hline 205992 & Benzo(b)fluoranthene \\
\hline 191242 & Benzo(ghi)perylene \\
\hline 207089 & Benzo $(k)$ fluoranthene \\
\hline 65850 & Benzoic acid \\
\hline 111911 & Bis (2-chloroethoxy)methane \\
\hline 111444 & Bis (2-ch1oroethy 1$)$ ether \\
\hline 108601 & Bis(2-chloroisopropyl) ether \\
\hline 117817 & Bis (2-ethylhexy1)phthalate \\
\hline 85687 & Butylbenzylphthalate \\
\hline 86748 & Carbazole \\
\hline 218019 & Chrysene \\
\hline 84742 & Di-n-butylphthalate \\
\hline 117840 & Di-n-octylphthalate \\
\hline 53703 & Dibenz $(\mathrm{a}, \mathrm{h})$ anthracene \\
\hline
\end{tabular}

\begin{tabular}{|c|c|c|c|c|c|c|c|c|}
\hline \multicolumn{3}{|c|}{ Customer } & \multirow{2}{*}{$\begin{array}{l}\text { Dilution } \\
\text { Factor }\end{array}$} & \multirow{2}{*}{$\begin{array}{l}\text { Lower } \\
\text { Limit }\end{array}$} & \multirow[b]{2}{*}{ Result } & \multirow{2}{*}{$\begin{array}{l}\text { EPA } \\
\text { Qual }\end{array}$} & \multirow[b]{2}{*}{ Confidence } & \multirow[b]{2}{*}{ Unit } \\
\hline Hт & Lim & $\mathrm{Fn}$ & & & & & & \\
\hline \multirow[t]{51}{*}{--} & $\cdots$ & -- & -.....- & ---- & ----- & --- & 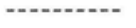 & $\cdots$ \\
\hline & & & 3 & 240 & 1200 & $u$ & & $u g / L$ \\
\hline & & & 3 & 270 & 1200 & $\mathrm{u}$ & & $\mathrm{ug} / \mathrm{L}$ \\
\hline & & & 3 & 140 & 1200 & $u$ & & $\mathrm{ug} / \mathrm{L}$ \\
\hline & & & 3 & 410 & 1200 & $u$ & & $u g / L$ \\
\hline & & & 3 & 420 & 1200 & u & & $u g / L$ \\
\hline & & & 3 & 460 & 1200 & u & & $u g / L$ \\
\hline & & & 3 & 250 & 1200 & u & & $\mathrm{ug} / \mathrm{L}$ \\
\hline & & & 3 & 230 & 1200 & u & & $\mathrm{ug} / \mathrm{L}$ \\
\hline & & & 3 & 240 & 1200 & u & & $u g / L$ \\
\hline & & & 3 & 340 & 6000 & u & & $\mathrm{ug} / \mathrm{L}$ \\
\hline & & & 3 & 320 & 1200 & $u$ & & $u g / L$ \\
\hline & & & 3 & 230 & 2400 & u & & $\mathrm{ug} / \mathrm{L}$ \\
\hline & & & 3 & 210 & 1200 & $u$ & & $\mathrm{ug} / \mathrm{L}$ \\
\hline & & & 3 & 240 & 1200 & $u$ & & $\mathrm{ug} / \mathrm{L}$ \\
\hline & & & 3 & 250 & 1200 & u & & $\mathrm{ug} / \mathrm{L}$ \\
\hline & & & 3 & 160 & 1200 & u & & $\mathrm{ug} / \mathrm{L}$ \\
\hline & & & 3 & 240 & 1200 & $u$ & & $\mathrm{ug} / \mathrm{L}$ \\
\hline & & & 3 & 280 & 1200 & u & & $\mathrm{ug} / \mathrm{L}$ \\
\hline & & & 3 & 190 & 1200 & $u$ & & $u g / L$ \\
\hline & & & 3 & 120 & 2400 & $u$ & & $u g / L$ \\
\hline & & & 3 & 280 & 2400 & $u$ & & $u g / L$ \\
\hline & & & 3 & 520 & 1200 & $u$ & & $u g / L$ \\
\hline & & & 3 & 160 & 1200 & $u$ & & $u g / L$ \\
\hline & & & 3 & 130 & 1200 & $u$ & & $u g / L$ \\
\hline & & & 3 & 240 & 1200 & u & & $u g / L$ \\
\hline & & & 3 & 160 & 1200 & u & & $\mathrm{ug} / \mathrm{L}$ \\
\hline & & & 3 & 260 & 2400 & $u$ & & $\mathrm{ug} / \mathrm{L}$ \\
\hline & & & 3 & 240 & 6000 & u & & $\mathrm{ug} / \mathrm{L}$ \\
\hline & & & 3 & 220 & 1200 & $u$ & & $\mathrm{ug} / \mathrm{L}$ \\
\hline & & & 3 & 250 & 1200 & $\mathrm{u}$ & & ug/L \\
\hline & & & 3 & 96 & 2400 & $\mathrm{u}$ & & ug/L \\
\hline & & & 3 & 130 & 1200 & $u$ & & $\mathrm{ug} / \mathrm{L}$ \\
\hline & & & 3 & 150 & 1200 & $u$ & & $u g / L$ \\
\hline & & & 3 & 300 & 6000 & $u$ & & $u g / L$ \\
\hline & & & 3 & 140 & 1200 & $u$ & & $u g / L$ \\
\hline & & & 3 & 110 & 1200 & $u$ & & $u g / L$ \\
\hline & & & 3 & 260 & 1200 & u & & $\mathrm{ug} / \mathrm{L}$ \\
\hline & & & 3 & 360 & 1200 & $u$ & & $\mathrm{ug} / \mathrm{L}$ \\
\hline & & & 3 & 350 & 1200 & u & & $\mathrm{ug} / \mathrm{L}$ \\
\hline & & & 3 & 460 & 6000 & u & & ug $/ \mathrm{L}$ \\
\hline & & & 3 & 130 & 1200 & u & & ug/L \\
\hline & & & 3 & 330 & 1200 & $\mathbf{u}$ & & $u g / L$ \\
\hline & & & 3 & 170 & 1200 & u & & $\mathrm{ug} / \mathrm{L}$ \\
\hline & & & 3 & 290 & 1200 & u & & $\mathrm{ug} / \mathrm{L}$ \\
\hline & & & 3 & 300 & 1200 & $u$ & & $u g / L$ \\
\hline & & & 3 & 150 & 1200 & u & & $u g / L$ \\
\hline & . & & 3 & 230 & 1200 & $u$ & & $\mathrm{ug} / \mathrm{L}$ \\
\hline & & & 3 & 160 & 1200 & u & & $\mathrm{ug} / \mathrm{L}$ \\
\hline & & & 3 & 330 & 1200 & U & & $\mathrm{ug} / \mathrm{L}$ \\
\hline & & & 3 & 380 & 1200 & u & & $\mathrm{ug} / \mathrm{L}$ \\
\hline
\end{tabular}

UNCLASSIFIED 


\section{UNCLASSIFIED}

LIMS 03.08 .048

04/30/2010 13:21

132649 Dibenzofuran

84662 Diethylphthalate

131113 Dimethylphthalate

$206440 \quad$ Fluoranthene

86737 Fluorene

118741 Hexachlorobenzene

$77474 \quad$ Hexachlorocyclopentadiene

67721 Hexachloroethane

193395 Indeno(1,2,3-cd)pyrene

78591 Isophorone

$621647 \quad$ N-Nitroso-di-n-propylamine

$62759 \quad \mathrm{~N}-\mathrm{Nit}$ rosodimethylamine

$86306 \quad \mathrm{~N}-\mathrm{Nit}$ trosodiphenylamine

91203 Naphthalene

$98953 \quad$ Nitrobenzene

87865 Pentachlorophenol

85018 Phenanthrene

108952 Pheno 1

129000 Pyrene
Analytical Chemistry organization (Quality Services)

Page 2 of 2

P.O. Box 2009 oak Ridge, TN 378318244 (865) 574-2284 official Report

Lab Smp1 Id: A100910402

Cust Smp1 Id: HFIR Nitric 200Mrad

$\begin{array}{lllll}3 & 220 & 1200 & \mathrm{U} & \mathrm{ug} / \mathrm{L} \\ 3 & 210 & 1200 & \mathrm{U} & \mathrm{ug} / \mathrm{L} \\ 3 & 240 & 1200 & \mathrm{U} & \mathrm{ug} / \mathrm{L} \\ 3 & 120 & 1200 & \mathrm{U} & \mathrm{ug} / \mathrm{L} \\ 3 & 180 & 1200 & \mathrm{U} & \mathrm{ug} / \mathrm{L} \\ 3 & 160 & 1200 & \mathrm{U} & \mathrm{ug} / \mathrm{L} \\ 3 & 1200 & \mathrm{U} & \mathrm{ug} / \mathrm{L} \\ 3 & 320 & 1200 & \mathrm{U} & \mathrm{ug} / \mathrm{L} \\ 3 & 360 & 1200 & \mathrm{U} & \mathrm{ug} / \mathrm{L} \\ 3 & 240 & 1200 & \mathrm{U} & \mathrm{ug} / \mathrm{L} \\ 3 & 170 & 1200 & \mathrm{U} & \mathrm{ug} / \mathrm{L} \\ 3 & 160 & 1200 & \mathrm{U} & \mathrm{ug} / \mathrm{L} \\ 3 & 360 & 1200 & \mathrm{U} & \mathrm{ug} / \mathrm{L} \\ 3 & 140 & 1200 & \mathrm{U} & \mathrm{ug} / \mathrm{L} \\ 3 & 230 & 1200 & \mathrm{U} & \mathrm{ug} / \mathrm{L} \\ 3 & 120 & 2400 & \mathrm{U} & \mathrm{ug} / \mathrm{L} \\ 3 & 130 & 1200 & \mathrm{U} & \mathrm{ug} / \mathrm{L} \\ 3 & 150 & 1200 & \mathrm{U} & \mathrm{ug} / \mathrm{L} \\ 3 & 220 & 1200 & \mathrm{U} & \end{array}$

Sample Test Comments: Target Compound(s) Initial Calibration exceeding 15\% RSD:

65-85-0 Benzoic acid

90-13-1 1-Chloronaphthalene

51-28-5 2,4-Dinitropheno]

100-02-7 4-Nitrophenol

92-87-5 Benzidine

Comment objects:

EPA Qualifiers:

U - Analyte analyzed for but undetected. Analyte result was below the contract required quantitation limit (CRQL).

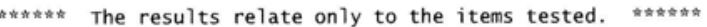

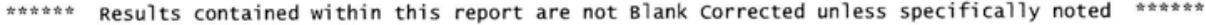

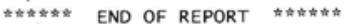

\section{UNCLASSIFIED}




\section{UNCLASSIFIED}

LIMS 03.08.048

04/30/2010 13:20
Analytical Chemistry organization (Quality Services)

P.O. Box 2009 oak Ridge, TN 378318244 (865) 574-2284

official Report
Page 1 of 2

Lab Smpl Id: A100910403

Project: L MS MPO SCHUH SVOA

Proj Mgr: L P BURNETT (865) 576-9141

Cust Smpl Id: HFIR Nitric

150 mracl

Customer: L P BURNETT Bldg 9995 MS-8189 (865) 576-9141

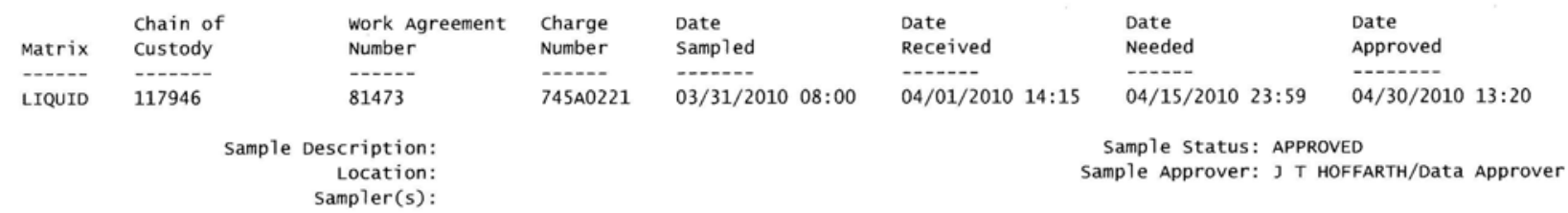

Customer comments:

Lab comments:

Comment objects:

$\ll<<$ Organic $\gg \gg>$

Test: SVOA8270 (Semi-volatile Organics by GC/MS)

Rpt Basis: AR

Analysis Method: SW846 8270C (Y50-AC-65-7316)

Approver: J T HOFFARTH/Data Approver

QC Batch/File: QC10118031/5362/53833
Prep Method/Date: Sw846 3580A 04/06/2010 10:00 Test Status: APPROVED

HT Deadline: 05/16/2010 23:59

Date Analyzed: 04/26/2010 23:23 Lab Group: OYGCMS

\begin{tabular}{|c|c|}
\hline Analyte Id & Analyte Name \\
\hline 120821 & 1,2,4-Trichlorobenzene \\
\hline 95501 & $\begin{array}{l}1,2,4 \text {-Trichlorobenzene } \\
1,2 \text {-Dichlorobenzene }\end{array}$ \\
\hline 122667 & 1,2-Diphenylhydrazine \\
\hline 541731 & 1,3-Dichlorobenzene \\
\hline 106467 & 1,4-Dichlorobenzene \\
\hline 95954 & 2,4,5-Trichlorophenol \\
\hline 88062 & 2,4,6-Trichlorophenol \\
\hline 120832 & 2,4-Dichlorophenol \\
\hline 105679 & 2,4-Dimethylphenol \\
\hline 51285 & 2,4-Dinitropheno 1 \\
\hline 121142 & 2,4-Dinitrotoluene \\
\hline 606202 & 2,6-Dinitrotoluene \\
\hline 91587 & 2-Ch1oronaphthalene \\
\hline 95578 & 2-Chlorophenol \\
\hline 534521 & 2-Methy $7-4,6$-dinitrophenol \\
\hline 91576 & 2-Methylnaphthalene \\
\hline 95487 & 2-Methylphenol \\
\hline 88744 & 2-Nitrobenzenamine \\
\hline 88755 & 2-Nitrophenol \\
\hline 91941 & 3, 3'-Dichlorobenzidine \\
\hline N2799 & 3- and 4- Methylphenol \\
\hline 99092 & 3-Nitrobenzenamine \\
\hline 101553 & 4-Bromopheny 1 pheny 1 ether \\
\hline 59507 & 4-Ch1oro-3-methy 1phenol \\
\hline 106478 & 4-Chlorobenzenamine \\
\hline 7005723 & 4-Ch1oropheny 1pheny 1 ether \\
\hline 100016 & 4-Nitrobenzenamine \\
\hline 100027 & 4-Nitrophenol \\
\hline 83329 & Acenaphthene \\
\hline 208968 & Acenaphthylene \\
\hline 62533 & Aniline \\
\hline 120127 & Anthracene \\
\hline 100516 & Benzenemethanol \\
\hline 92875 & Benzidine \\
\hline 56553 & Benzo (a) anthracene \\
\hline 50328 & Benzo(a)pyrene \\
\hline 205992 & Benzo(b)fluoranthene \\
\hline 191242 & Benzo(ghi)perylene \\
\hline 207089 & Benzo $(k)$ fluoranthene \\
\hline 65850 & Benzoic acid \\
\hline 111911 & Bis (2-chloroethoxy)methane \\
\hline 111444 & Bis (2-chloroethy 1 ) ether \\
\hline 108601 & Bis (2-chloroisopropy 1 ) ether \\
\hline 117817 & Bis(2-ethylhexyl)phthalate \\
\hline 85687 & Butylbenzylphthalate \\
\hline 86748 & Carbazole \\
\hline 218019 & Chrysene \\
\hline 84742 & Di-n-butylphthalate \\
\hline 117840 & Di-n-octylphthalate \\
\hline 53703 & Dibenz $(a, h)$ anthracene \\
\hline
\end{tabular}

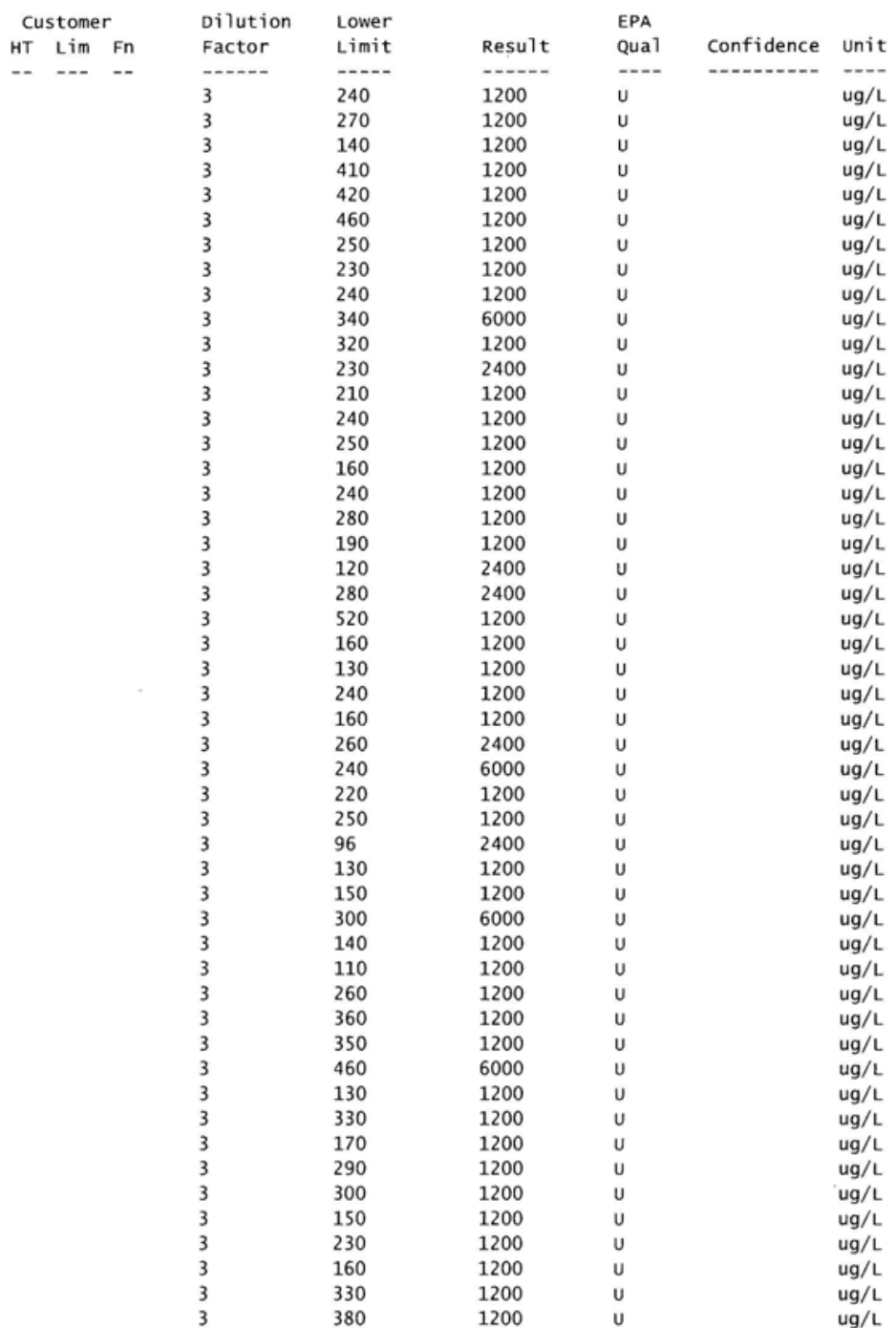

UNCLASSIFIED 


\section{UNCLASSIFIED}

LIMS 03.08.048

04/30/2010 13:20

$\begin{array}{ll}132649 & \text { Dibenzofuran } \\ 84662 & \text { Diethylphthalate } \\ 131113 & \text { Dimethylphthalate } \\ 206440 & \text { Fluoranthene } \\ 86737 & \text { Fluorene } \\ 118741 & \text { Hexachlorobenzene } \\ 77474 & \text { Hexachlorocyclopentadiene } \\ 67721 & \text { Hexachloroethane } \\ 193395 & \text { Indeno(1,2,3-cd)pyrene } \\ 78591 & \text { Isophorone } \\ 621647 & \text { N-Nitroso-di-n-propylamine } \\ 62759 & \text { N-Nitrosodimethylamine } \\ 86306 & \text { N-Nitrosodiphenylamine } \\ 91203 & \text { Naphthalene } \\ 98953 & \text { Nitrobenzene } \\ 87865 & \text { Pentachlorophenol } \\ 85018 & \text { Phenanthrene } \\ 108952 & \text { Phenol } \\ 129000 & \text { Pyrene } \\ & \\ \text { TIC Id } & \text { TIC Name } \\ ----- & \text {------ } \\ & \text { unknown }\end{array}$

Analytical Chemistry organization (Quality Services)

P.O. Box 2009 oak Ridge, TN 378318244 (865) 574-2284

Page 2 of 2 official Report

$\begin{array}{ll}220 & 1200 \\ 210 & 1200 \\ 240 & 1200 \\ 120 & 1200 \\ 180 & 1200 \\ 160 & 1200 \\ 620 & 1200 \\ 360 & 1200 \\ 240 & 1200 \\ 170 & 1200 \\ 160 & 1200 \\ 360 & 1200 \\ 140 & 1200 \\ 230 & 1200 \\ 120 & 1200 \\ 130 & 2400 \\ 150 & 1200 \\ 220 & 1200 \\ 270 & 1200 \\ & \\ \text { Retention } & \\ \text { Time } & \text { Unit } \\ --- & -\cdot-- \\ 6.59 & \text { min }\end{array}$

Lab Smp1 Id: A100910403

$\begin{array}{cr}u & \text { HFIR Nitric } 250 \mathrm{mad} \\ u g / L & 150 \mathrm{mrad}\end{array}$ $\mathrm{ug} / \mathrm{L}$

ug/L

$\mathrm{ug} / \mathrm{L}$

$u g / L$

$\mathrm{ug} / \mathrm{L}$

$u g / L$

$\mathrm{ug} / \mathrm{L}$

$u g / L$

$\mathrm{ug} / \mathrm{L}$

ug/L

$\mathrm{ug} / \mathrm{L}$

$u g / L$

$\mathrm{ug} / \mathrm{L}$

$u g / L$

$\mathrm{ug} / \mathrm{L}$

$\mathrm{ug} / \mathrm{L}$

$u g / L$

$\mathrm{ug} / \mathrm{L}$

Estimated EPA

\begin{tabular}{lll} 
Conc & Qual & Unit \\
\hdashline 620 & J & ug/L
\end{tabular}

Sample Test Comments: Target compound(s) Initial calibration exceeding 15\% RSD:

$\begin{array}{ll}65-85-0 & \text { Benzoic acid } \\ 90-13-1 & 1-\text { Chloronaphthalene } \\ 51-28-5 & 2,4-D i n i t r o p h e n o l \\ 100-02-7 & 4-N i t r o p h e n o l \\ 92-87-5 & \text { Benzidine } \\ \text { The mean Initial Calibration RSD }=10.6\end{array}$

comment objects :

EPA Qualifiers:

J - Estimated value.

U - Analyte analyzed for but undetected. Analyte result was below the contract required quantitation limit (CRQL).

\section{UNCLASSIFIED}

